\title{
Raman Investigations Of Strained Ferroelectrics
}

\author{
BY \\ Benjamin James Wylie-Van Eerd
}

\begin{abstract}
A thesis
submitted to the Victoria University of Wellington in fulfilment of the requirements for the degree of Doctor of Philosophy
\end{abstract}

Victoria University of Wellington

(2013) 


\section{Abstract}

This describes research done on a variety of ferroelectric systems over the course of three years during the Ph.D. programme at Victoria University of Wellington. The majority of the work involved using Raman spectroscopy to investigate the lattice dynamics of the ferroelectric materials studied, and by this method measuring the structural phase diagrams in the ferroelectrics.

Three material systems were investigated. The first was bulk ceramics of the solid solution of $\mathrm{Na}_{0.5} \mathrm{Bi}_{0.5} \mathrm{TiO}_{3}$ and $\mathrm{BaTiO}_{3}$ (BNT-BT). The second material was $\mathrm{PbTiO}_{3}(\mathrm{PT})$ nanowires prepared by annealing 'PX' phase lead titanate in air. In these samples we found a tensile strain caused by nanoscopic voids in the wires. The third material studied was thin films of $\mathrm{SrTiO}_{3}$ (STO) grown epitaxially on lattice mismatch substrates. This introduced strain into the system.

In the BNT-BT system, temperature dependent Raman spectra were taken of the various samples. From the spectra, it was discovered that the structural phase transitions of the material did not perfectly correspond to the electrical phase transitions. For one significant phase boundary, no structural change occurs, only a loss of long-range order. The local sensitivity of the Raman spectroscopy technique allowed this to be found. As a consequence of this, no tricritical point is found in the phase diagram of BNT-BT. It was also found that poling the sample in electrical fields shifted the morphotropic phase boundary between $5 \%$ and $6 \%$ Ba-substitution about 1\% towards the high-substitution side, but otherwise did not affect the phase diagram.

In the PT nanowires system, temperature dependent Raman spectroscopy and scanning electron microscopy were performed to measure the spectra of single nanowires. A large enhancement of the ferroelectric phase transition temperature was discovered. The enhancement was found to be dependent on the nanowire diameter, with peak enhancements of over $100 \mathrm{~K}$ measured in wires close to $125 \mathrm{~nm}$. Wires both larger and smaller than this showed smaller degrees of enhancement. It is proposed that the enhancement is caused by tensile strain developed in the wires during their synthesis, where they were transformed from a low-density phase into a high-density phase. 
In the STO thin films system, temperature dependent ultraviolet Raman spectra and x-ray diffraction spectra were measured to establish a relationship between the biaxial strain developed in the films and their phase diagrams. The XRD found strain in the films of similar substrate which was inversely proportional to thickness up to a threshold point. Beyond that point, there is a discontinuity and additional thickness of film is grown without strain. The strain in the lower layer remains constant. The UV Raman spectroscopy method was able to enhance the signal such that thin films with weak signals could be measured. The spectra showed signs of a phase transition in all of the films. In one film enough of the spectral features were visible to characterise the low temperature phase as the orthorhombic ferroelectric phase of STO. The transition temperature varied from sample to sample, and a relationship between the biaxial strain and the transition temperature was seen. 
Dedication

To Damalaya and Francesco van Eerd, my mama and papa

The older and wiser I grow,

the more I come to value the childhood you gave me. 


\section{Contents}

$\begin{array}{ll}\text { Abstract } & \text { iii }\end{array}$

Abbreviations used in this thesis $\quad$ xi

List of Figures $\quad$ xxix

1 Introduction $\quad 1$

1.1 What is Ferroelectricity? . . . . . . . . . . . . . . 2

1.1.1 Ferroelectric Materials . . . . . . . . . . . . . . 5

1.1.2 Properties of Ferroelectrics . . . . . . . . . . . . . . . 7

1.1.3 Ferroelectric Phase Transitions . . . . . . . . . . . . . . . . 10

1.1.4 Ferroelectrics in Technology . . . . . . . . . . . . . . . . . 14

1.2 Raman Spectroscopy . . . . . . . . . . . . . . . . . . . . 15

1.2.1 What is a phonon? . . . . . . . . . . . . . 17

1.2.2 Raman scattering . . . . . . . . . . . . . . . . . 17

1.2.3 Spectroscopic method . . . . . . . . . . . . . . 22

1.2.4 Soft mode spectroscopy . . . . . . . . . . . . . . . . . 24

2 Background Information $\quad 29$

$2.1 \mathrm{Bi}_{0.5} \mathrm{Na}_{0.5} \mathrm{TiO}_{3}-\mathrm{BaTO}_{3}$ system . . . . . . . . . . . . . . . 29

2.2 Lead titanate nanowires . . . . . . . . . . . . . . . . . . 32

2.3 STO Thin Films . . . . . . . . . . . . . . . . . . . . 34

2.3.1 Enhancing Raman signals from thin films using UV excitation 38

2.3.2 Raman features of STO . . . . . . . . . . . . . . . 40

2.3.3 Specific examples . . . . . . . . . . . . . . . . 52

2.3.4 General themes in STO systems . . . . . . . . . . . . 80

2.3.5 Summary of Raman signatures of ferroelectricity in STO . . 83 
3 Experimental methods and materials $\quad 85$

3.1 Sample information . . . . . . . . . . . . . . . . . . 85

3.1 .1 BNT-BT system . . . . . . . . . . . . . . . 85

3.1.2 PT nanowires system . . . . . . . . . . . . . . 86

3.1 .3 STO thin films . . . . . . . . . . . . . . 87

3.2 Experimental methods . . . . . . . . . . . . . . . . . 88

$3.2 .1 \quad$ BNT-BT . . . . . . . . . . . . . . . . . 88

3.2 .2 PT nanowires . . . . . . . . . . . . . . . . . . . 88

3.2 .3 Strained STO thin films . . . . . . . . . . . . . . 91

3.3 Experimental apparatus . . . . . . . . . . . . . . . 93

3.3.1 Discussion of spectrometer systems . . . . . . . . . . 93

3.3.2 Discussion of temperature control systems . . . . . . . . . 96

4 Results - BNT-BT 101

4.1 Raman study . . . . . . . . . . . . . . . . . . . . . . 101

4.2 Phase diagram . . . . . . . . . . . . . . . . . . . . 102

4.3 Poling study on BNT-BT . . . . . . . . . . . . . . 107

4.4 Effect of poling on the phase diagram . . . . . . . . . . . . . . 110

4.5 BNT-BT conclusions . . . . . . . . . . . . . . . . . 112

5 Results - PT Nanowires $\quad \mathbf{1 1 5}$

5.1 Sample 1 - >100 nm PT nanowires . . . . . . . . . . . . . 115

5.1 .1 Initial SEM imaging . . . . . . . . . . . . . . . . . 115

5.1 .2 Raman features . . . . . . . . . . . . . . . . . 116

5.1.3 Temperature dependence and phase transition . . . . . . . . 120

5.2 Sample 2 - <100 nm PT nanowires . . . . . . . . . . . . . . . . . 122

5.2 .1 Initial SEM imaging . . . . . . . . . . . . . . . . . . . 122

5.2 .2 Raman spectra . . . . . . . . . . . . . . . . . . . . 124

5.2.3 Final SEM and morphology change . . . . . . . . . . . 129

5.3 Discussion of enhanced $\mathrm{T}_{C} \ldots \ldots \ldots$. . . . . . . . . . . 139

5.4 Conclusions - PT nanowires . . . . . . . . . . . . . . . . . . . . 142

6 Results - STO thin film system $\quad 145$

6.1 STO on STO . . . . . . . . . . . . . . . . . . 145

6.1.1 Sample STO003 - STO/STO. . . . . . . . . . 146

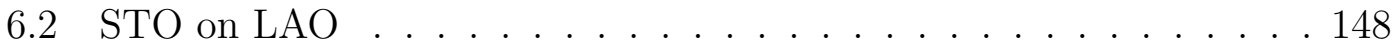


6.2.1 Sample 267, $100 \mathrm{~nm}$ STO/LAO: spectra in helium cryostat . 148

6.2.2 Sample STO267, $100 \mathrm{nmSTO/LAO:} \mathrm{spectra} \mathrm{in} \mathrm{nitrogen} \mathrm{cryo-}$ stat . . . . . . . . . . . . . . . 157

6.2.3 Sample 263, $30 \mathrm{nmSTO/LAO:} \mathrm{spectra} \mathrm{in} \mathrm{helium} \mathrm{cryostat} \mathrm{.} \mathrm{.} 163$

6.2.4 Samples STO263 and STO267: Overall intensity anomaly . . 166

6.2.5 Summary of results - STO on LAO . . . . . . . . . . . . . . 167

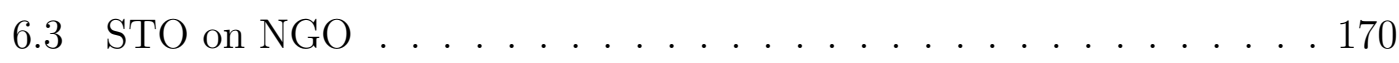

6.3.1 Sample STO264: $50 \mathrm{~nm}$ STO/NGO: spectra in helium cryostat170

6.3.2 Summary of results - STO on NGO . . . . . . . . . . . 181

6.4 STO on LSAT . . . . . . . . . . . . . . . . . . . . . . . 181

6.4.1 Sample STO006: $105 \mathrm{~nm}$ STO/LSAT measured in helium cryostat . . . . . . . . . . . . . . . . 181

6.4.2 Sample STO005: $140 \mathrm{~nm}$ STO/LSAT measured in helium cryostat . . . . . . . . . . . . . . . 183

6.4.3 Sample STO004: $60 \mathrm{~nm}$ STO/LSAT measured in helium cryostat . . . . . . . . . . . . . 186

6.4.4 Summary of results - STO on LSAT . . . . . . . . . . . . 189

6.5 XRD measurements . . . . . . . . . . . . . . . . . 190

$6.6100 \mathrm{~cm}^{-1}$ feature . . . . . . . . . . . . . . . . . 192

6.7 Strain - phase transition relationship . . . . . . . . . . . . . . 194

6.8 STO strained thin films - conclusions . . . . . . . . . . . . . . . 197

7 Conclusions 201

7.1 BNT-BT - summary of results . . . . . . . . . . . . . 201

7.2 BNT-BT - suggested further work . . . . . . . . . . . . . 202

7.3 PT nanowires - summary of results . . . . . . . . . . . . . . . 202

7.4 PT nanowires - suggested further work . . . . . . . . . . . . . 203

7.5 STO thin films - summary of results . . . . . . . . . . . . . . 204

7.6 STO thin films - suggested further work . . . . . . . . . . . 205

$\begin{array}{ll}\text { A Paper in submission concerning PT nanowires } & 207\end{array}$

A.1 Nanopore-induced negative-pressure ferroelectric enhancement in freestanding $\mathrm{PbZrO}_{3}$ nanowires . . . . . . . . . 207

A.2 Abstract . . . . . . . . . . . . . . . . . . . . 208

A.3 Article . . . . . . . . . . . . . . . . . . . 208

A.4 References of this appendix . . . . . . . . . . . . . 216 
B Semi-classical equation describing resonant Raman scattering $\quad 219$

$\begin{array}{ll}\text { Bibliography } & 221\end{array}$ 


\section{Abbreviations used in this thesis}

- AFD: Anti-ferrodistortive. Refers to a distortion of the perovskite unit cell whereby neighboring oxygen octahedra rotate in opposite directions around the c-axis

- AFM: Atomic force microscope/microscopy. Analytical technique to create topological profiles at very small length scales

- BNT: Sodium bismuth titanate, $\mathrm{BiNaTiO}_{3}$. One of the end members of the BNT-BT system

- BNT-BT: Bismuth sodium titanate mixed with barium titanate, $\left(\mathrm{Na}_{0.5} \mathrm{Bi}_{0.5}\right)_{1-\mathrm{x}} \mathrm{Ba}_{\mathrm{x}} \mathrm{TiO}_{3}$. A lead-free ferroelectric and one of the materials studied in this thesis

- BT: Barium titanate, $\mathrm{BaTiO}_{3}$. One of the end members of the BNT-BT system

- DSO: Dysprosium scandate, $\mathrm{DyScO}_{3}$. Substrate material used by [1] to create room temperature ferroelectric STO thin films

- FDTD: Finite-difference time-domain. A method of computational modelling

- FEM: Finite element modelling. A method of computational modelling

- FE: Ferroelectric. Possessing an ordered arrangement of electric dipole moments that may be switched by an external electric field

- IR: Infra-red. Part of the electromagnetic spectrum 
- KLT: Potassium lithium tantalate, $\left(\mathrm{K}_{0.5} \mathrm{Li}_{0.5}\right) \mathrm{TaO}_{3}$. A perovskite ferroelectric studied in $[2]$

- LAO: Lanthanum aluminate, $\mathrm{LaAlO}_{3}$. A substrate material used in this study with a small lattice mismatch with STO

- $\mathrm{LO}_{x}$ : Longitudinal optical mode $x$. A vibrational mode of solids that can interact with light. Numbering is from low to high frequency

- LSAT: Lanthanum-strontium aluminate-tantalate, $\left(\mathrm{LaAlO}_{3}\right) \cdot\left(\mathrm{SrAl}_{0.5} \mathrm{Ta}_{0.5} \mathrm{O}_{3}\right)$. A substrate material used in this study with a small lattice mismatch with STO

- MPB: Morphotropic phase boundary. This is a kind of phase boundary that is highly sensitive to composition, and relatively insensitive to temperature. Vertical in a temperature-composition phase diagram

- NGO: Neodymium gallate, $\mathrm{NdGaO}_{3}$. A substrate material used in this study with a small lattice mismatch with STO

- PMR: Polar micro-regions. These are volumes inside STO and other materials that are ferroelectric in the paraelectric phase of the material (i.e. before the ferroelectric transition occurs)

- PT: Lead titanate, $\mathrm{PbTiO}_{3}$. A strong ferroelectric material, still used in some applications today

- PX: This refers to the specific phase of lead titanate studied in[3]

- PZT: Lead zirconate titanate, $\mathrm{Pb}_{1-\mathrm{x}} \mathrm{Zr}_{\mathrm{x}} \mathrm{TiO}_{3}$. The most technologically used ferroelectric material

- SEM: Scanning electron microscope/microscopy. Analytical technique to create images at very small length scales

- SERS: Surface enhanced Raman spectroscopy. A technique for enhancing Raman spectroscopy.

- SRO: Strontium ruthanate. Material often used as an electrical contact in STO heterostructures. Another perovskite, SRO has a very similar lattice constant to STO. 
- STO: Strontium titanate, $\mathrm{SrTiO}_{3}$. An 'incipient' ferroelectric and one of the materials studied in this thesis

- TEM: Transmission electron microscope/microscopy. Analytical technique to create images at extremely small length scales

- TERS: Tip enhanced Raman spectroscopy. A combination of atomic force microscopy plus SERS

- $\mathrm{TO}_{x}$ : Transverse optical mode $x$. A vibrational mode of solids that can interact with light. Numbering is from low to high frequency

- UV: Ultraviolet. Part of the electromagnetic spectrum

- XRD: X-ray diffraction. A common technique for structural analysis 


\section{List of Figures}

1.1 Left: cartoon of randomly aligned magnetic dipoles in a regular array such as a crystal. The material is paramagnetic. Right: The intensity of the magnetic field of such a collection of dipoles, $|B|$, plotted against distance from the surface of the material, d. The field strength drops away extremely rapidly with distance. . . . . . 3

1.2 Left: An ordered array of dipole moments such as in a single domain of a ferromagnetic material. Right: the magnetic field approaches that of a single stronger dipole in the geometric centre. . . . . . . . 4

1.3 The perovskite crystal structure, with a-site cations in red, b-site cations in green, and oxygen in blue. Shown left - cubic symmetry, and right - tetragonal symmetry. Note that neither of these crystal structures is ferroelectric. . . . . . . . . . . . . . . . 6

1.4 Two displacements of the b-site cation in tetragonal perovskites that can lead to ferroelectricity. . . . . . . . . . . . . .

1.5 A ferroelectric hysteresis loop. /itE is the electric field applied to the ferroelectric, and /itP is the resulting polarisation of the ferroelectric

1.6 Cartoon depiction of piezoelectricity. Applied stress results in a potential across the material, and conversely applied potential results in strain. . . . . . . . . . . . . . . . . . . . . . . 11

1.7 The distinctive 'Curie Weiss' behaviour of the dielectric permittivity of a ferroelectric material. The peak in the permittivitytemperature graph corresponds to the ferroelectric phase transition temperature. Both permittivity and inverse permittivity are shown (figure reproduced with permission from [5]) . . . . . . . . . . . 12 
1.8 Phase diagram of lead zirconate titanate, emphasizing the morphotropic phase boundary (Reprinted by permission from Macmillan Publishers Ltd: Nature[9], copyright 2004). . . . . . . . . . . . .

1.9 A Raman scattering event: incoming light interacts with a vibrational mode of the molecule, and is scattered with altered energy (frequency). Compare this with Rayleigh scattering, where scattered energy is the same as incoming. . . . . . . . . . . . . 16

1.10 Phonons. Shown above is a normal vibrational mode of a $1 \mathrm{~d}$ crystal. Below - two different energy levels of this vibration, wavefunction vs displacement. Left - the first excited state left (one phonon) and right - the second excited state (two phonons). . . . . . . . . 18

1.11 In Stokes scattering a vibrational mode in the material gains an energy level (phonon) and the scattered light has lower frequency (energy) . . . . . . . . . . . . . . . . 19

1.12 In anti-Stokes scattering a vibrational mode in the material loses an energy level (phonon) and the scattered light has higher frequency (energy). . . . . . . . . . . . . . . . . .

1.13 Raman spectrum of silicon. The Rayleigh scattered light can be seen in the centre, the Stokes scattered light on the right and the anti-Stokes scattered light on the left. Note that this means that light of higher frequency is on the left of the spectrum, not the right - ie, Raman shift is equal to the negative of the change in frequency. This is the usual way that Raman spectra are displayed. The arrows in the spectrum show the cut off points provided by the notch filter. The Rayleigh scattered light is in fact much stronger than is shown here - it is being heavily attenuated.

1.14 Soft phonon mode for the cubic-tetragonal phase transition in a perovskite. The atomic displacement vectors for the vibration match those for the transition. . . . . . . . . . . . . . . .

1.15 Real data showing the frequency of the anti-ferrodistortive soft mode in $\mathrm{SrTiO}_{3}$. The temperature $T_{a}$ in the figure is the temperature of antiferrodistortive phase transition in STO. Note that the frequencies are in this case measured by infrared spectroscopy. Figure reproduced with permission from [14] . . . . . . . . . . . . 26 
2.1 An early (1991) phase diagram of BNT-BT produced by bulk electronic measurements. Note the morphotropic phase boundary between the $\mathrm{F}_{\alpha}$ and $\mathrm{F}_{\beta}$ phases, and the triple point between those two phases and the AF phase (the top phase marked $\mathrm{P}$ is a paraelectric cubic phase). Although this phase diagram is now considered substantially incorrect, I include it to illustrate the state of knoledge of BNT-BT prior to my research. Copyright 1991 The Japan Society of Applied Physics, figure reproduced with permission from [16]. . .

2.2 Structure of 'PX' phase of lead titanate, found in nanowires. Left: space filling geometrical model, and right: balls and sticks atomic model. Note the unusual gap in the structure, giving significantly reduced density in this phase. Reprinted (adapted) with permission from [3]. Copyright 2011 American Chemical Society. . . . . . . . .

2.3 SEM image of PT nanowires produced via annealing of the less dense PX phase nanowires. The nanowire morphology can be seen to be retained through the annealing process. . . . . . . . . . . 34

2.4 Raman spectrum of tetragonal (and ferroelectric) lead titanate for reference. The cubic (paraelectric) phase of lead titanate has no first-order peaks. (C)2007 IEEE, Figure reproduced with permission from $[17] . \ldots \ldots \ldots \ldots \ldots \ldots$

2.5 Left: crystal structure in the cubic phase of STO, z-projection. Right: crystal structure of the AFD phase of STO characterised by anti-phase rotation of adjacent oxygen octahedra about the z-axis. Note the doubling of the unit cell caused by this transition. . . . . .

2.6 Temperature dependent Raman spectra of STO. The spectra have been vertically offset for ease of viewing. The second-order features and R-point modes activated by the AFD transition can be seen in these spectra. Reprinted with permission from [27]. Copyright 1968, American Institute of Physics. . . . . . . . . . . . . . .

2.7 Raman spectra of STO showing the $\mathrm{E}_{g}$ and $\mathrm{A}_{1 g}$ components of the AFD soft mode (and the hard mode at $150 \mathrm{~cm}^{-1}$ ). The spectra have been vertically offset for ease of viewing. Figure reproduced with permission from $[19] \ldots \ldots \ldots \ldots$. . . . . . . . . . . . . . . . . .

2.8 Temperature dependence of the AFD soft mode frequency. Reprinted figure with permission from[37]. Copyright 1968 by the American Physical Society. . . . . . . . . . . . . . . . . . 
2.9 Raman spectra showing the splitting of the $\mathrm{E}_{g}$ component of the ferroelectric soft mode upon phase transition to the orthorhombic ferroelectric phase, shown in this case in ${ }^{18} \mathrm{O}$ substituted STO. The spectra have been vertically offset for ease of viewing. Reprinted figure with permission from[38]. Copyright 2005 by the American Physical Society. . . . . . . . . . . . . . . . . . 46

2.10 Raman spectra of STO showing first-order scattering modes (labeled in red). The spectra have been vertically offset for ease of viewing. Reprinted figure with permission from[42]. Copyright 2010 by the American Physical Society. . . . . . . . . . . . . . . . . . . . 49

2.11 Close up of the $\mathrm{TO}_{2}$ mode in polycrystalline thin films. The spectra have been vertically offset for ease of viewing. Note the strongly asymmetric shape of the mode in this spectrum. Figure reproduced

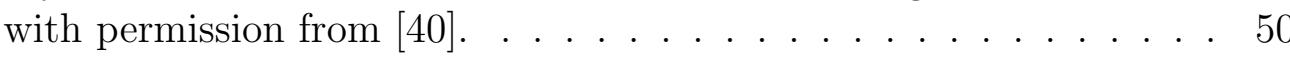

2.12 Temperature dependent Raman spectra of polycrystalline STO thin films. The spectra have been vertically offset for ease of viewing. In this Raman spectrum, the $\mathrm{TO}_{3}$ mode can be seen. Figure repro-

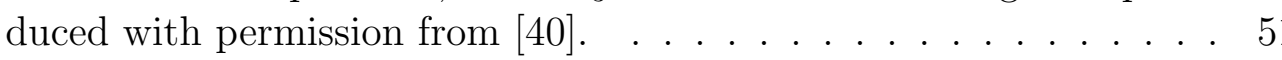

2.13 Left: Raw spectra and Right: frequency-temperature dependence of ferroelectric soft mode in Ca-substituted STO. The spectra have been vertically offset for ease of viewing. In this material, there is a ferroelectric phase below $20 \mathrm{~K}$. The softening to near zero and the splitting into three components below $\mathrm{T}_{C}$ can be seen in these figures (splitting of the $\mathrm{E}_{g}$ component of the AFD soft mode can also been seen in this figure). In the right hand panel of this figure, FMR refers to Raman scattering activated by ferroelectric microregions. Figure reproduced with permission from [43] . . . . . . . . . . . 52

2.14 Close up of the low-frequency range of Raman spectra on pure single crystal STO. The spectra have been vertically offset for ease of viewing. The shoulder that develops on the low-frequency side of the $74 \mathrm{~cm}^{-1}$ peak and subsequently softens is scattering from the ferroelectric soft mode. This mode softening is incomplete down to $0 \mathrm{~K}$ in pure STO. Reprinted with permission from [27]. Copyright 1968, American Institute of Physics. . . . . . . . . . . 53 
2.15 Temperature dependence of the first-order mode intensity in Casubstituted STO. Open squares: $\mathrm{TO}_{1}$ mode, open circles: $\mathrm{TO}_{2}$ mode, closed circles: dielectric loss in this material for comparison. Note the rapid rise to saturation in a curve somewhat like a Fermi-Dirac distribution. In the remainder of this thesis, this kind of temperature dependence will be referred to as an S-shaped curve. Figure reproduced with permission from [43] . . . . . . . . . 56

2.16 First-order mode intensity vs temperature in STO with higher Casubstitution levels. Both $\mathrm{TO}_{2}$ and $\mathrm{TO}_{4}$ modes are displayed, as marked Left: $6 \% \mathrm{Ca}$, Right: $12 \% \mathrm{Ca}$. Note the same S-shaped curve being shown as in 2.15 , but spread out much broader in temperature. Figure reproduced with permission from $[19] \ldots$. . . . . . . . .

2.17 Dielectric permittivity of STO with various levels of Ca-substitution, as marked. $0.2 \%$ substitution is sufficient to induce a ferroelectric phase. The phase transition temperature and peak permittivity both increase up to $1.07 \%$. Subsequently, the peak permittivity falls and broadens, and the transition temperature stagnates at around 37 K. Reprinted figure with permission from[47]. Copyright 1984 by the American Physical Society. . . . . . . . . . . . . . . . . 59

2.18 Raman spectra of ${ }^{18} \mathrm{O}$ substituted STO. The spectra have been vertically offset for ease of viewing. Left: The activation of the ferroelectric soft mode can be seen. These spectra collected in $x(y y)-x$ geometry. Right, a) $23 \%{ }^{18} \mathrm{O}$ substituted STO and b) $32 \%{ }^{18} \mathrm{O}$ substituted STO. These spectra collected in $x(y z)-x$ The splitting of the AFD soft mode can be seen in b) compared to the spectra resulting from no phase transition in a). Both the activation of the ferroelectric soft mode and the splitting of the AFD soft mode are strong indicators of a ferroelectric phase transition. Reprinted figure with permission from[38]. Copyright 2005 by the American Physical Society. . . . . . . . . . . . . . . .

2.19 Temperature dependent Raman spectra of STO ceramics. The spectra have been vertically offset for ease of viewing. The R-point AFD modes can be seen, as can the $\mathrm{TO}_{2}$ and $\mathrm{TO}_{4}$ first-order modes. The intense mode marked $\mathrm{X}$ is thought to be either a component of the AFD soft mode, or the ferroelectric soft mode. Figure reproduced with permission from $[36] . \ldots \ldots \ldots$. . . . . . . . . 63 
2.20 Figure showing the decay over time of the piezoelectric force microscopy signal from STO, comparison of strain and strain-free. Reprinted figure with permission from[42]. Copyright 2005 by the American Physical Society. . . . . . . . . . . . . . . .

2.21 Raman spectra of non-stoichiometric single-crystal thin films of STO. The spectra have been vertically offset for ease of viewing. $x$ is the $\mathrm{Sr}$ excess (or deficiency, for negative values). Spectra were taken at $10 \mathrm{~K}$, and non-stoichiometric samples show first-order modes. Additionally, there is a marked difference in the AFD modes between Sr-deficient and Sr-excess films. Figure reproduced with permission from [50]. . . . . . . . . . . . . . . . . 68

2.22 Temperature-strain phase diagram of STO. The graph was created from thermodynamic analysis. The range of transition is due to conflicting reports on parameters used in the modeling.Reprinted by permission from Macmillan Publishers Ltd: Nature[1], copyright

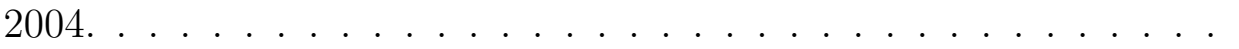

2.23 Figure showing temperature dependence of several factors. Upper: asymmetry factor, $q$, of $\mathrm{TO}_{2}$ mode in their spectra. Middle: Dielectric permittivity, showing a very slight ferroelectric-type peak. Lower: first-order mode intensity, compared with bulk. Reprinted figure with permission from[38]. Copyright 2005 by the American Physical Society. . . . . . . . . . . . . . . .

2.24 Raman spectra of STO with various levels of oxygen vacancies, all taken at $10 \mathrm{~K}$. The spectra have been vertically offset for ease of viewing. The spectra are identified by the amount of time spend in a reducing atmosphere. Reprinted figure with permission from[53]. Copyright 2007 by the American Physical Society. . . . . . . . . . . 76

2.25 Temperature dependence of first-order scattering in KLT. Above: 1\% Li, Below: $4 \% \mathrm{Li}$. the $4 \% \mathrm{KLT}$ has a ferroelectric phase transition at about $50 \mathrm{~K}$, and one can see the spectral changes associated with it. $1 \%$ KLT is not ferroelectric. Reprinted figure with permission from[2]. Copyright 1992 by the American Physical Society.

3.1 Schematic of UV optical path between sample and spectrometer. This figure is not drawn to scale, but it shows the light cone collected, the magnification caused by the positioning and numerical aperture of the chosen UV lens, and the filling of the spectrometer first mirror. Distances shown are measurements in $\mathrm{mm}$. . . . . . . . 
3.2 Sources of line broadening in Raman spectroscopy. Left: Dispersion. Higher dispersion = greater resolution. Right: focal length. Greater focal length $=$ greater resolution. . . . . . . . . . . . . . 95

3.3 More sources of broadening in Raman spectroscopy. The fabricated spectra have been vertically offset for ease of viewing. Left: Detection pixel size. Smaller pixels = greater resolution. Right:Slit width. Smaller slit width $=$ greater resolution. BUT - also smaller throughput. . . . . . . . . . . . . . . . 96

4.1 Spectra of BNT-BT taken by the author in a previous study. The spectra have been vertically offset for ease of viewing. The top (red) spectra are taken at high temperatures, and the bottom (black spectra) are taken at low temperatures. Left: 5\% Ba-substituted BNT, Right: 6\% Ba-substituted BNT. The arrows show features in the spectra including a discontinuous drop in the frequency of the $150 \mathrm{~cm}^{-1}$ mode, and the splitting of one peak into two near $300 \mathrm{~cm}^{-1}$. The spectra show no phase transition from low to high temperature in the $6 \%$ BT sample, in opposition to what was found for bulk dielectric measurements. . . . . . . . . . . . . . . . . . 103

4.2 Temperature-dependent spectra of BNT-BT taken by the author in a previous study. The spectra have been vertically offset for ease of viewing. The spectra show the $5 \%$ BT sample being taken up in temperature over the phase transition between rhombohedral and tetragonal symmetries. Inset: Fitted peak frequencies of the Raman modes seen in the spectra. A discontinuous change can be seen in these at $380 \mathrm{~K} . \ldots \ldots$. . . . . . . . . . . . . . 104

4.3 Phase diagram of BNT-BT based on Raman measurements of our samples. Zone I is ferroelectric and rhombohedral, zone II is ferroelectric and tetragonal, zone III is tetragonal, but is not ferroelectric. (Open symbols measure phase transitions as a function of temperature, closed symbols show implied phase transitions - 5\% sample is Rhombohedral and 6\% sample is Tetragonal at this temperature). Note the lack of any Raman signal of a phase transition between zones II and III, and contrast this with Fig. 2.1, the phase diagram in Takenaka et al. [16]. There is no structural change accompanying this electrical phase transition. . . . . . . . . . 106 
4.4 Raman spectrum of the poled $6 \%$ sample compared with spectra of the depoled samples at 5\% (Rhombohedral) and 6\% (Tetragonal). The spectra have been vertically offset for ease of viewing. The poled sample is dissimilar to the depoled sample of the same composition. Reprinted figure with permission from[4]. Copyright 2010 by the American Physical Society. . . . . . . . . . . . . . 108

4.5 Raman spectrum of the poled $6 \%$ sample compared to a line created from a 80/20 mix of spectra of depoled samples. The spectra have been vertically offset for ease of viewing. This suggests that the $6 \%$ poled sample is in a mixed-phase. Reprinted figure with permission from[4]. Copyright 2010 by the American Physical Society. . . . . . 109

4.6 Dielectric permittivity of the $6 \%$ samples - poled and depoled. A temperature of depoling is clearly seen and is marked. Below this temperature, there is a notable difference in the permittivity for poled and unpoled samples. Reprinted figure with permission from[4]. Copyright 2010 by the American Physical Society. . . . . . . . . . . 111

5.1 SEM images of PT nanowires showing morphology. The wires shown in d) e) and f) were used to obtain Raman spectra and have diameters of $650 \mathrm{~nm} 250 \mathrm{~nm}$ and $125 \mathrm{~nm}$ respectively. . . . . . . 117

5.2 Raman spectrum of a large PT nanowire used in this sample. The first order peaks are clear in the tetragonal, ferroelectric phase. . . . 118

5.3 Temperature dependent Raman spectra of a large PT nanowire. The spectra have been vertically offset for ease of viewing. This wire shows the tetragonal-cubic phase transition at $500{ }^{\circ} \mathrm{C}$, the same as bulk PT. . . . . . . . . . . . . . . . . . . . . . . . 119

5.4 Raman spectra of three wires at three temperatures. The spectra have been vertically offset for ease of viewing. In blue: $125 \mathrm{~nm}$ wire, red: $250 \mathrm{~nm}$ wire and black: $650 \mathrm{~nm}$. At $475^{\circ} \mathrm{C}$ all three wires are in the tetragonal phase. at $500^{\circ} \mathrm{C}$, the smaller two wires are tetragonal and the largest wire is cubic. At $600^{\circ} \mathrm{C}$, the larger two wires are cubic and only the smallest wire is still in the tetragonal phase. . . 121

5.5 Temperature dependence of soft mode frequency of the three wires showing softening. Recall that the $125 \mathrm{~nm}$ wire is still tetragonal at the highest temperature measured. Its soft mode frequency at $600^{\circ} \mathrm{C}$ is still above the frequency at which the mode disappeared in the other wires measured. . . . . . . . . . . . . . . . 123 
5.6 SEM images showing the smallest wires studied. A red circle in part a) shows on this figure the location and size of the laser spot used to make Raman measurements b) measurements made on the crossed wires can be used to make comparisons for the insufficiently resolved wires. . . . . . . . . . . . . . . . . . 125

5.7 SEM images showing the smallest wires measured. These are the highest resolution images of the wires measured prior to measurement.126

5.8 Raw temperature dependent Raman spectra collected from the region of sub $100 \mathrm{~nm}$ PT nanowires. The spectra have been vertically offset for ease of viewing. The spectra are dominated by the central frequency noise. The features above $400 \mathrm{~cm}^{-1}$ are sapphire lines from the substrate, and PT peaks can be seen at 85 and $287 \mathrm{~cm}^{-1}$. 127

5.9 Between $450{ }^{\circ} \mathrm{C}$ and $500{ }^{\circ} \mathrm{C}$, the spectral lines from the PT disappear, suggesting a tetragonal to cubic phase transition. The spectra have been vertically offset for ease of viewing. This temperature is not elevated as are the other nanowire $\mathrm{T}_{C} \mathrm{~s}$ studies in this work. . . 128

5.10 Temperature dependent Raman spectra of the region of sub $100 \mathrm{~nm}$ PT nanowires. The spectra have been vertically offset for ease of viewing. These spectra were produced by fitting a Lorentzian line to the central frequency noise and subtracting it from the spectrum. In these spectra the PT lines can be clearly seen, and the frequencies measured. Note the softening of the ferroelectric soft mode below $100 \mathrm{~cm}^{-1}$. The top line (black) is of the PT wires at $500^{\circ} \mathrm{C}$, and the disappearance of the PT lines can be clearly seen. . . . . . . . . . . 130

5.11 Temperature dependence of the ferroelectric soft mode in sub $100 \mathrm{~nm}$ PT wires (black squares) compared with $125 \mathrm{~nm}$ and $250 \mathrm{~nm}$ wires. The open symbol indicates that we cannot be certain whether this spectrum was affected by the morphology change or not. For the filled symbols, we can be confident that they were not. If the spectrum at $450 \mathrm{~K}$ is trusted, then this strongly indicates a comparatively lower $\mathrm{T}_{C}$ and the elevated frequency. If not, then the evidence for a lower $\mathrm{T}_{C}$ is not so clear. . . . . . . . . . . . . . . . 131

5.12 SEM images of Sub $100 \mathrm{~nm}$ PT nanowires before and after Raman measurement. The before picture has been rotated such that the substrate has the same alignment in both images. The wires have transformed in lumps. . . . . . . . . . . . . . . . . . 133 
5.13 SEM images of Sub $100 \mathrm{~nm}$ PT nanowires before and after Raman measurement. The before picture has been rotated such that the substrate has the same alignment in both images. Seen in this image is a region of nanowires that was not within the Raman laser spot. It can be seen that the nanowires have also disappeared from this region, ruling out the influence of the laser in the morphology change. 135

5.14 SEM images of Sub $100 \mathrm{~nm}$ PT nanowires before and after Raman measurement. The before picture has been rotated such that the substrate has the same alignment in both images. In this image we can see strong evidence of migration of the smaller wires into clumps on the surface of larger ones. Note also though, the vertically aligned wire in the middle of the after image. Although reduced in length, this particular smaller wire has retained its wire morphology. . . . . 136

5.15 SEM images of Sub $100 \mathrm{~nm}$ PT nanowires after Raman measurement. Another image taken far from the site of Raman spectroscopy showing changed morphology of the PT nanowires. In this case, there was not significant migration of lumps onto the larger PT structure shown. . . . . . . . . . . . . . . . . 137

5.16 SEM image of $125 \mathrm{~nm}$ PT nanowire measured in this study. Note the small lumps visible on the substrate near the wire. Although smaller than those in the sub $100 \mathrm{~nm}$ PT nanowires sample, they could be a sign that there were once smaller PT wires on this substrate.138

5.17 Raman spectra of sub $100 \mathrm{~nm}$ PT nanowires, both at $400{ }^{\circ} \mathrm{C}$, and both with a central frequency Lorentzian subtracted from the raw spectra. The spectra have been vertically offset for ease of viewing. The lower(black) spectrum was taken while temperature was rising, on the way up to $500^{\circ} \mathrm{C}$. The upper (red) spectrum is the same sample taken on the way down, after the spectral change seen at $500{ }^{\circ} \mathrm{C}$. The position of the ferroelectric soft mode is noticeably different in the two spectra, while the other features are identical. . 140

5.18 SEM images of three different PT nanowires of different diameters, and data for the tetragonality of these wires vs. wire diameter. The thinnest wire shows no circular voids. There are some voids in the middle wire, and many more in the thicker wire. The tetragonality of the wires is found to be enhanced. The enhancement is dependent on the diameter of the wires, with maximum enhancement of twice the bulk tetragonality found in wires of diameter of $115 \mathrm{~nm}$. . . . . 141 
6.1 Temperature dependent Raman spectra of STO/STO thin film. The spectra are vertically offset for ease of viewing. The spectra match very well those of single crystal STO samples, with mostly second order features visible. The TO and LO modes are absent. The arrows mark out the ferroelectric soft mode scattering, similar to [27] and thought to be caused by polar microregions. . . . . . . . . 147

6.2 Raman spectra of sample STO267 (red), and the LAO substrate on which it is grown (black), both at room temperature. Substrate peaks and second order features from STO can be seen in the thin film. . . . . . . . . . . . . . . . . . . . 149

6.3 Raman spectra of STO267 and LAO taken at $9 \mathrm{~K}$. At this temperature, there are many more lines in the film spectrum than in the substrate.

6.4 Temperature dependent Raman spectra of STO267 showing the emergence of the first order modes in STO. The spectra are vertically offset for ease of viewing. . . . . . . . . . . . . . . . . . 151

6.5 Temperature dependent Raman spectra of STO267, showing a close up on the $100 \mathrm{~cm}^{-1}$ feature. The spectra are vertically offset for ease of viewing. . . . . . . . . . . . . . . . . . . . . . . 152

6.6 Temperature dependent XRD spectra showing the emergence of the characteristic AFD superlattice peak at about $400 \mathrm{~K}$. The spectra have been vertically offset for ease of viewing. This work was not my contribution. . . . . . . . . . . . . . . . . 153

6.7 Temperature dependence of the three first-order mode peak heights visible in STO267. The vertical scales are not the same in each of the three plots. It can be seen that all three peaks intensities have the same temperature dependence. . . . . . . . . . . . . . 156

6.8 Temperature dependence of the $100 \mathrm{~cm}^{-1}$ and $\mathrm{TO}_{2}$ mode peak heights in sample STO267, taken from Lorentzian fits to the data. The intensities of the modes vary almost identically with temperature, which is a strong indication that the $100 \mathrm{~cm}^{-1}$ feature has the same origin as the other first order modes. . . . . . . . . . . . . . . 158

6.9 Raman spectra of sample STO267 at $80 \mathrm{~K}$ taken in two different experimental configurations - in black, the nitrogen cryostat and in red, the helium cryostat. There are some differences between the two spectra that can be attributed to experimental broadening, differences in throughput and differences in the volume being measured.160 
6.10 Temperature dependence of the $\mathrm{TO}_{4}$ peak height in sample STO267 as measured in the nitrogen cryostat. Note the long non-zero tail that remains above $200 \mathrm{~K}$, visible in this configuration due to the better surface selectivity. . . . . . . . . . . . . . . . 161

6.11 Temperature dependent Raman spectra of STO267 taken in the nitrogen cryostat showing the $\mathrm{TO}_{4}$ peak (marked with an arrow) remaining to high temperatures. The spectra have been offset both horizontally and vertically for ease of viewing . . . . . . . . . 162

6.12 Temperature dependence of the $\mathrm{TO}_{4}$ peak height from measurements in both the nitrogen and helium cryostats, scaled to match each other between $80 \mathrm{~K}$ and $160 \mathrm{~K}$. . . . . . . . . . . . 163

6.13 Raman spectra of sample STO263, and of the LAO substrate on which it is grown. Both spectra are taken at $9 \mathrm{~K}$, and this sample shows the same STO peaks that sample STO267 does, though they are weaker.

6.14 Comparison of the Raman spectra at $9 \mathrm{~K}$ of the $100 \mathrm{~nm}$ and $30 \mathrm{~nm}$ STO layers on LAO. The relative intensity of the different STO modes varies between the two samples. . . . . . . . . . . 165

6.15 Temperature dependence of the $\mathrm{TO}_{4}$ mode in sample STO263, put together from the nitrogen and helium cryostat measurements as for the previous sample. In this sample, a saturation of the mode intensity at low temperatures is much more obvious. . . . . . . . . 166

6.16 Temperature dependence of the $\mathrm{TO}_{4}$ modes of both STO on LAO samples. Measurements in both the nitrogen and helium cryostats are used to make this figure. The same pattern is seen in both samples, shifted $50 \mathrm{~K}$ higher in temperature in the thinner film, sample STO263. . . . . . . . . . . . . . . . . 167

6.17 Temperature dependent Raman spectra of sample STO267 taken in the nitrogen cryostat. The spectra have been horizontally and vertically offset for ease of viewing. Note the dip in intensity of all modes - both STO and substrate - in the middle of the temperature series, at about $180 \mathrm{~K} . \ldots \ldots$. . . . . . . . . . . 168

6.18 Peak heights of an STO and a substrate mode, variation with temperature. Left panel: Descending temperature. Right panel: ascending temperature. The dip in intensity of all modes is seen only with rising temperature. . . . . . . . . . . . . . . . 169 
6.19 Raman spectra of sample STO264 compared to the NGO substrate on which it is grown. In this case, there are very few modes which are seen in the sample and not the substrate - a feature near $40 \mathrm{~cm}^{-1}$ and one at $800 \mathrm{~cm}^{-1}$. Note that this spectrum of STO on NGO has a lower resolution $-10 \mathrm{~cm}^{-1}$ rather than the usual $5 \mathrm{~cm}^{-1} \ldots \ldots . .171$

6.20 Temperature dependent Raman spectra of NGO substrate. The spectra have been vertically offset for ease of viewing. The peaks at $180 \mathrm{~cm}^{-1}$ and $550 \mathrm{~cm}^{-1}$ marked with arrows not only have the same frequencies as the the $\mathrm{TO}_{2}$ and $\mathrm{TO}_{4}$ modes of STO, but they also show a very similar variation with temperature. It will be very difficult to use these peaks in the sample to infer anything about

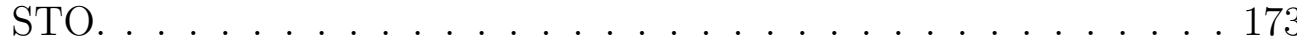

6.21 Temperature dependent Raman spectra of sample STO264, close up on $\mathrm{LO}_{4}$ peak. The spectra have been vertically offset for ease of viewing. The peak changes more sharply with temperature in this sample than in the other STO samples studied so far. . . . . . . . . 174

6.22 Temperature dependent Raman spectra of sample STO264, close up on the $\mathrm{TO}_{3}$ peak. The spectra have ben vertically offset for ease of viewing. This peak can be seen emerging from above substrate lines from about $159 \mathrm{~K}$. In this sample, $\mathrm{TO}_{3}$ has a different temperature dependence than the other TO and LO modes in STO. . . . . . . . 175

6.23 Figure showing fitting parameters of $\mathrm{LO}_{4}$ peak and underlying second order features. In black squares: fiting with all parameters free. Above $150 \mathrm{~K}$ the model becomes poor. This can be seen in the rapidly rising width parameter, blue circles. In red triangles is fitting with the width parameter constrained. The result is a better, though still not perfect representation of the $\mathrm{LO}_{4}$ peak intensity. . . 177

6.24 Temperature dependence of vibrational mode intensities in sample STO264. Note the difference between the $\mathrm{TO}_{3}$ and $\mathrm{TO}_{4}$ modes, and compare the changes at the dotted blue line in this figure to the changes at $50 \mathrm{~K}$ in Fig. 6.25 . . . . . . . . . . . . . . . . . 178

6.25 Temperature dependence of first-order scattering in KLT. Above: 1\% Li, Below: $4 \% \mathrm{Li}$. the $4 \% \mathrm{KLT}$ has a ferroelectric phase transition at about $50 \mathrm{~K}$, and one can see the spectral changes associated with it. Similar effects are seen in the Raman spectra for STO264 see Fig. 6.24. 1\% KLT is not ferroelectric. Figure reproduced with permission from $[2] \ldots \ldots$. . . . . . . . . . . . . 179 
6.26 Temperature dependent Raman spectra of the $35 \mathrm{~cm}^{-1}$ feature in sample STO264. The spectra have been vertically offset for ease of viewing. Note the splitting of one peak into two peaks accompanied by a sharp increase in low-frequency scattering between $160 \mathrm{~K}$ and 140 K, as marked by the arrows. . . . . . . . . . . . . . . . . . 180

6.27 Raman spectra of sample STO006 - $105 \mathrm{~nm}$ of STO grown on LSAT, and of the LSAT substrate by itself. The spectra were both taken at 8 K. . . . . . . . . . . . . . . . . . 182

6.28 Temperature dependent Raman spectra of sample STO006. The spectra have been vertically offset for ease of viewing. The emergence of the $\mathrm{TO}_{2}$ and $\mathrm{TO}_{4}$ modes together with the $100 \mathrm{~cm}^{-1}$ feature can be seen here.

6.29 Comparison of the Raman spectra of $105 \mathrm{~nm}$ STO grown on LSAT to $140 \mathrm{~nm}$ STO grown on LAO. Spectra are shown at five different temperatures across the range where first order scattering emerges, and each temperature is vertically offset for ease of viewing. Lighter colours are the thicker layer, darker colours are the thinner layer. The temperature dependence of the $\mathrm{TO}_{2}$ and $\mathrm{TO}_{4}$ modes is very similar between the two samples.

6.30 Temperature dependence of the first-order STO modes in $105 \mathrm{~nm}$ and $140 \mathrm{~nm}$ of STO grown on LSAT.

6.31 Raman spectra of sample STO004, a thinner film of STO grown on LSAT compared with the substrate. Only the $\mathrm{TO}_{2}$ mode is clearly seen above the substrate lines in this sample. . . . . . . . . . 188

6.32 Temperature dependence of the $\mathrm{TO}_{2}$ mode intensity in sample STO004. The transition is relatively sharp and at a relatively high temperature compared to the thicker STO/LSAT samples. . . . . . . . . 189

6.33 Temperature dependence of the $\mathrm{TO}_{2}$ mode intensity in all three STO/LSAT samples. The similarity of the two thicker films, and the contrast with the thinnest film can be seen. . . . . . . . . . . 190

6.34 Room temperature XRD spectra of STO thin films grown to various thicknesses on LSAT. A spectrum of STO/STO is provided for reference. The out-of plane lattice constant is measured in these spectra, and the variation of the lattice constant with film thickness cna be seen. There is clearly a correlation between strain and thickness, and there is also clearly a two-phase growth mode in the thickest sample. . . . . . . . . . . . . . . . . . . . 193 
6.35 Example of how $\mathrm{T}_{C}$ for the STO thin films was calculated. A tangent to the polar amplitude vs. T curve was drawn at the steepest point, and a horizontal line was drawn at the low amplitude saturation point (dotted red lines). The intercept of these two lines was taken as the phase transition temperature. . . . . . . . . . . 196

6.36 Phase diagram of biaxially strained STO. The phases marked and the grey shaded regions between them are copied from the theory presented in [1]. Red squares show calculated phase transition temperatures as found by the Raman spectroscopy measurements in this thesis. Orange bars show the temperature range over which the polar Raman features are changing in intensity. This may indicate a growth of polar volume, an increase in polar order parameter or a combination of the two. . . . . . . . . . . . . . . . 198

A.1 SEM image of the perovskite PT nanowires. b, Low-magnification TEM image showing the closed nanosized pores in the nanowires. c, High resolution image revealing that the monocrystallinity is not affected by the pores. d, TEM image of the area with $90^{\circ}$ domains. e, $c / a$ versus the thickness of the nanowires. . . . . . . . . 210

A.2 Spectra on single wires of diameters 125,250 and $650 \mathrm{~nm}$, at temperatures spanning the Curie temperature. The lowest frequency $\mathrm{E}(1 \mathrm{TO})$ feature was followed to investigate the diameter-dependent Curie temperature. The occasional peak around $40-50 \mathrm{~cm}^{-1}$ is spurious, possibly from the substrate. b, The frequency of the $\mathrm{E}(1 \mathrm{TO})$ line plotted vs temperature for the three wires, showing the line falling to $55 \mathrm{~cm}^{-1}$ before disappearing at $\mathrm{T}_{C}$. The thinner wires have a clear enhancement of the ferroelectric temperature range. . . 211

A.3 Temperature dependence of lattice parameters (a) and c/a (b) of the multiple-wire sample compared with the standard bulk data (ref. 17). The simulated lattice parameters and $c / a$ of PT under $0.4 \mathrm{Gpa}$ hydrostatic tensile stress are also included (see the text for further discussions). . . . . . . . . . . . . . . . . . . 213

A.4 a-c, TEM images showing the pore distribution in the nanowires with different thicknesses. d, Enhanced $c / a$ (red curve with square markers) of PT under hydrostatic tensile stress simulated by the first-principles calculations (the curve is reconstructed according to the data in ref. 9) and upshift of $\mathrm{T}_{C}$ (solid black curve) of PT under hydrostatic tensile stress predicted by the LGD theory. . . . . . . . 214 


\section{Chapter 1}

\section{Introduction}

This thesis is an experimental investigation of several systems of ferroelectric materials under conditions of strain. In particular it is concerned with the effect that strain has on the structural phase transitions of the studied materials.

Raman spectroscopy is the principal tool used to measure the structural phase transitions in the ferroelectric materials that are studied. Raman measures directly the energy of lattice vibrations, which can subsequently be linked to the structural properties of the materials.

In the remainder of this chapter, I will provide some theoretical and practical background information on the phenomenon of ferroelectricity, and on the technique of Raman spectroscopy.

Chapter two will provide background information on the particular materials that are studied in this thesis. The sodium bismuth titanate - barium titanate mixed ceramic system is the first, and it is a ferroelectric that is not under conditions of strain. In studies of this material, we can clearly see the methods of Raman spectroscopy, the importance of phase transitions and of soft modes applied in a simple material of scientific interest. The second material system isnanowires of lead titanate. In these samples, there is a tensile strain in the wires, which enhances their ferroelectric properties. The last material system is thin films of strontium titanate. Biaxial compressive strain is applied to the thin films by growing them on a substrate which has a lattice constant slightly smaller than that of strontium 
titanate.

Chapter three reports the samples used, and the experimental methods used in this thesis. It includes discussions about the Raman spectrometers and temperature control systems used in the thesis, comparing them and justifying the experimental choices made.

Chapters four, five and six report the results of the experiments performed on the mixed ceramic system, the nanowires and the thin films respectively. It discusses the interpretation of the Raman and other experimental results in terms of the structural phase transitions of the materials, and the role of strain in these systems where applicable.

In chapter seven I summarise the results of this work, and conclude with my ideas for further study leading on from this research.

\subsection{What is Ferroelectricity?}

Ferroelectricity is the electric analogue to the magnetic property of ferromagnetism. It is a property of materials that can retain an electric polarisation in the absence of an external electric field, and that can have the direction of that polarisation set by an external field.

Each electron and most nuclei have an intrinsic magnetic dipole moment. In a solid, one can visualise a large array of point magnetic dipoles coming from these particles. In most materials, the coupling between neighboring dipole moments is weak, and thermal energy will randomise the direction of the dipoles even down to $0 \mathrm{~K}$. Such materials are called paramagnetic. This is illustrated in Fig. 1.1. At points sufficiently far from the dipole array, the magnetic field drops off far quicker than the $r^{-3}$ dependence found from a single dipole, rapidly becoming undetectably small. This is because the contributions from all of the differently oriented dipoles become approximately equal in magnitude, and in net they cancel each other out.

Imagine now a material in which it is energetically favourable for neighboring 


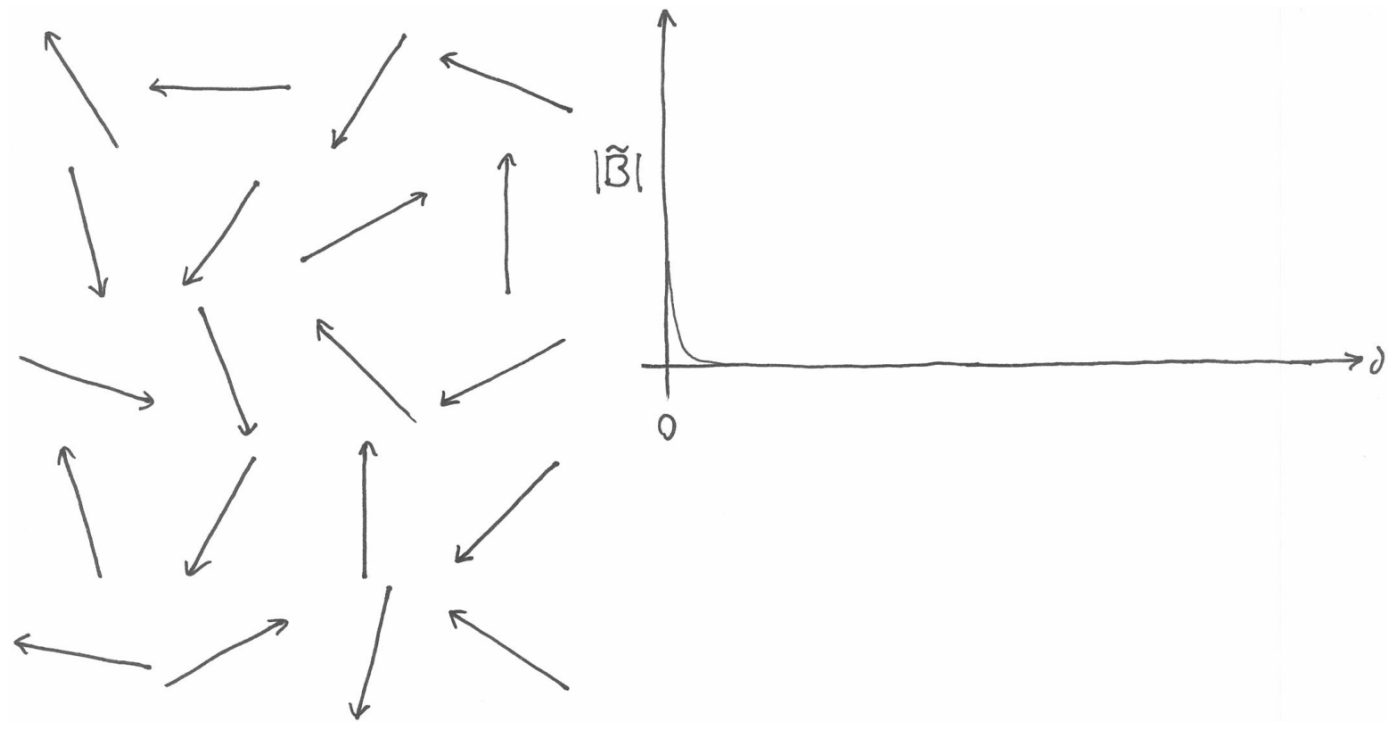

Figure 1.1: Left: cartoon of randomly aligned magnetic dipoles in a regular array such as a crystal. The material is paramagnetic. Right: The intensity of the magnetic field of such a collection of dipoles, - B - , plotted against distance from the surface of the material, d. The field strength drops away extremely rapidly with distance.

dipoles to align. In this case, large coherent domains are formed in which all of the dipoles point in the same direction. This is illustrated in Fig. 1.2. There is a huge difference in the magnetic field we measure - now at distances far from the array, the field measured is a sum without cancellation of all of the dipoles, and the measured field becomes very much like the far field of a single stronger dipole. Arrays with this property are called ferromagnetic.

The reality is more complicated, because it does cost energy to maintain a magnetic field such as this. So in order to minimise energy, one domain of single orientation does not extend over the whole crystal. Rather, many domains are formed. The domains are large compared to the dipole-dipole distance, but small compared to the dimensions of the whole crystal, and although all the dipoles in a single domain are similarly aligned, neighboring domains may point in different directions. Energy is lost as the macroscopic magnetic field is reduced, but gained as nearest-neighbor dipoles point in different directions. Eventually an optimal configuration of domains is formed, which still has a dominant dipole direction, and hence net magnetisation. 

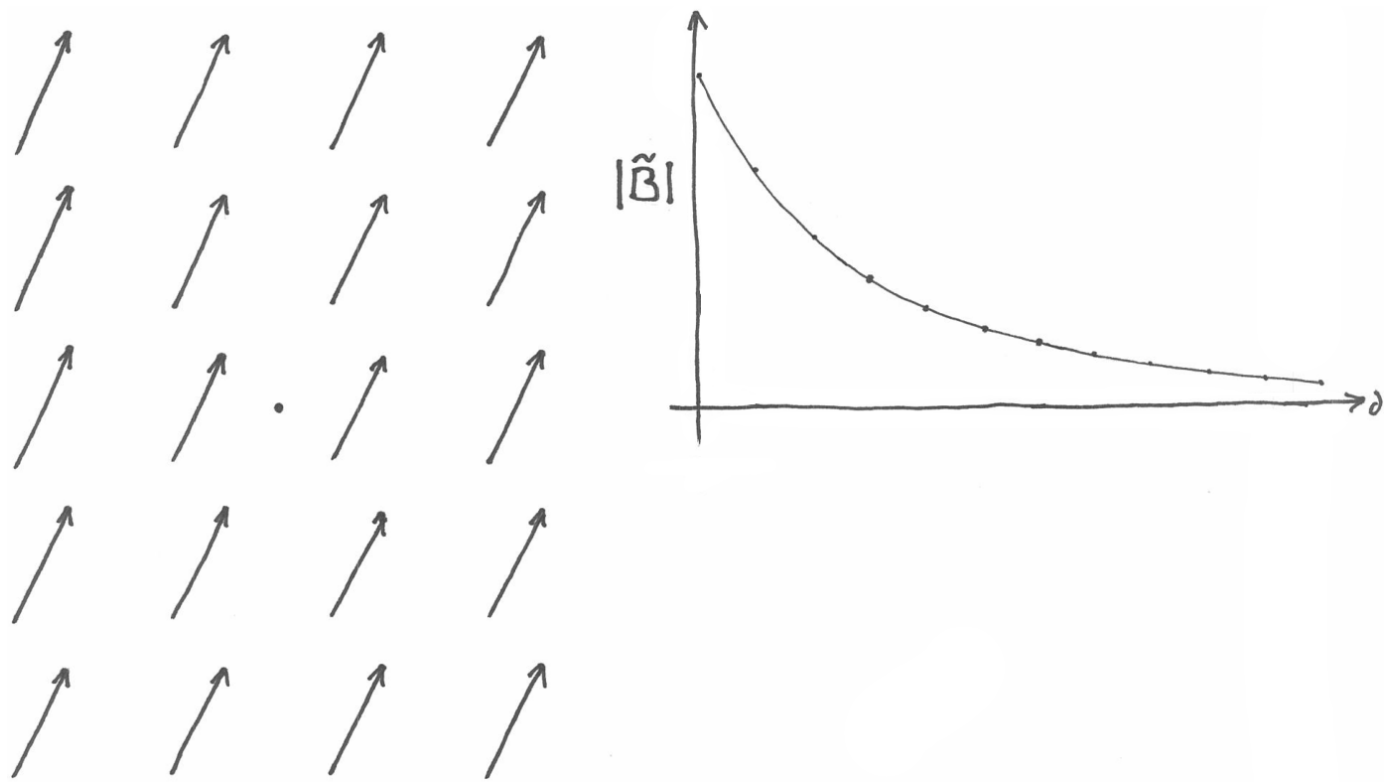

Figure 1.2: Left: An ordered array of dipole moments such as in a single domain of a ferromagnetic material. Right: the magnetic field approaches that of a single stronger dipole in the geometric centre.

Applying an external magnetic field to a ferromagnetic material will cause the magnetic dipoles of the domains to align with it. When the external magnetic field is removed, some domains will relax back into disordered directions, but the dominant polarisation of the material will now point in the direction that the external field did.

The necessary properties for a material to be ferromagnetic are: The ability to have magnetic dipoles (and therefore a magnetic field) in the absence of an external magnetic field; The ability of individual magnetic dipoles to form ordered arrays with other similar dipoles; And the ability of these dipoles to switch directions or rotate in response to an external magnetic field. With these properties, the material can hold a magnetic field of its own in the absence of an external magnetic field, and the direction of this internal magnetic field can be set by external poling.

Ferroelectricity is the same as ferromagnetism, except that one requires electric dipoles instead of magnetic ones, resulting in an electric field instead of a magnetic one. However, whereas magnetic dipoles are relatively easy to come across - many common metals such as iron for example have net magnetic dipoles - electric dipoles are much rarer in nature. While a magnetic dipole is a property that a single 
particle (such as an electron) can posess, any electric dipole requires necessarily an interaction of two particles to create a single dipole element - and they must be different types of particle, at that! The phenomenon is therefore more complex.

\subsubsection{Ferroelectric Materials}

The most common ferroelectrics are crystals with an asymmetric distribution of positive and negative charges in the unit cell. The uneven distribution creates a separation of charge, and as a consequence each unit cell carries a net electric dipole moment. These dipoles are the units of the array. Of the 32 crystal point groups, 10 fulfill the requirement of holding a net dipole. If the dipole can be switched, then the crystal is ferroelectric. Note that this switching is not trivial - it involves the movement of at least one ion core from one lattice position into another.

For example, I'll take the perovskite crystal structure as our basis (as this is the structure of the most common ferroelectrics, including those studied in this work). The unaltered structure is centrosymmetric at each atomic site. Moving from cubic to tetragonal or rhombohedral does not in itself alter the centrosymmetry (even though it creates unique axes). However, phase transitions from cubic to tetragonal or rhombohedral are often coincident with ferroelectric distortions. Fig. 1.3 shows the perovskite crystal structure, in cubic and tetragonal symmetries. A simple breaking of centrosymmetry might be the translation of the B-site cation along one of the [100] directions. After this change, the crystal no longer has inversion symmetry at any point. Instead, we have a unique axis along the [100] direction, in which there is a non-zero dipole moment. Looking at it in a different way, it has become impossible to construct a primitive unit cell in which the sum of ionic charge times vector distance over the unit cell is zero. $\mathrm{BaTiO}_{3}$ is an example of a material which achieves ferroelectricity via this particular ionic displacement.

Not all ferroelectrics have this particular displacement. For example, the rhombohedral phase in lead zirconate titanate(PZT), a material studied in this work, is ferroelectric because of a displacement of B-site cations in one of the [111] (pseudocubic) directions. Of course, one could still pole this crystal in a [100] direction. In this case, the dipoles in each unit cell would rotate to point in one of the four 

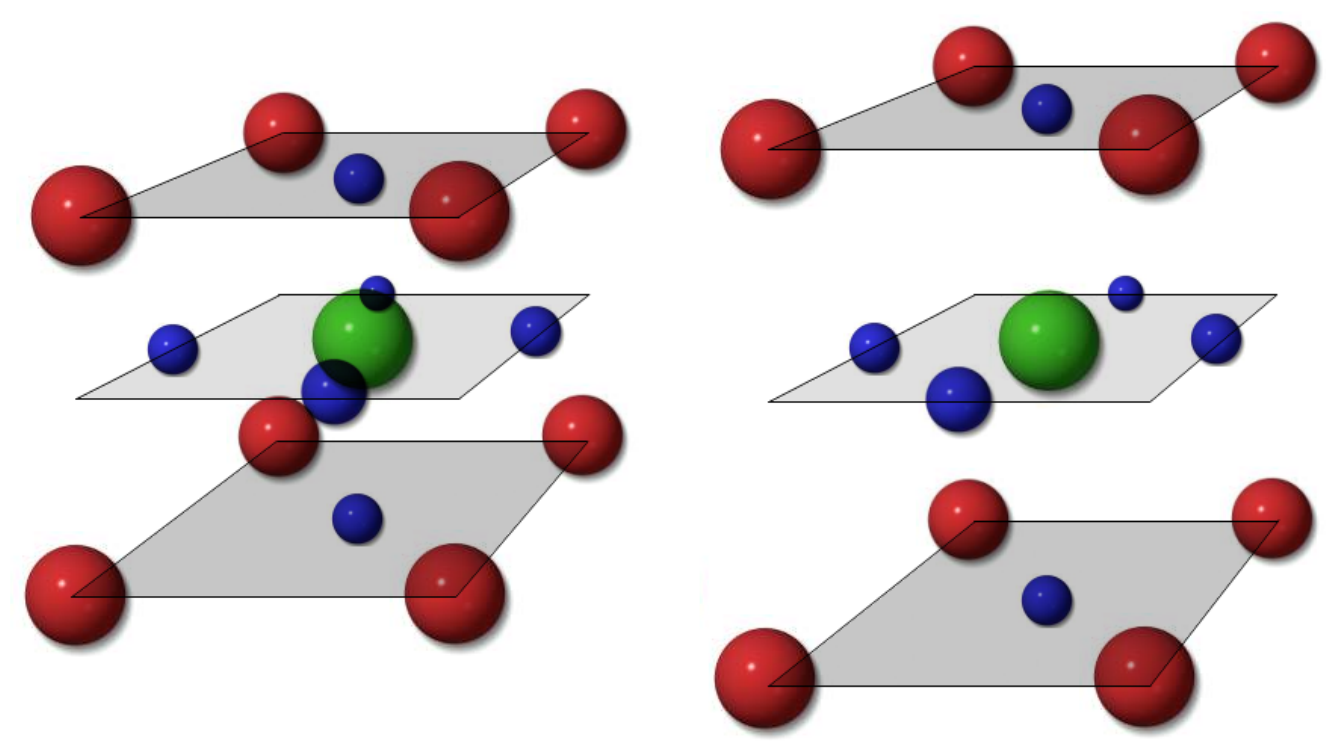

Figure 1.3: The perovskite crystal structure, with a-site cations in red, b-site cations in green, and oxygen in blue. Shown left - cubic symmetry, and right tetragonal symmetry. Note that neither of these crystal structures is ferroelectric. 

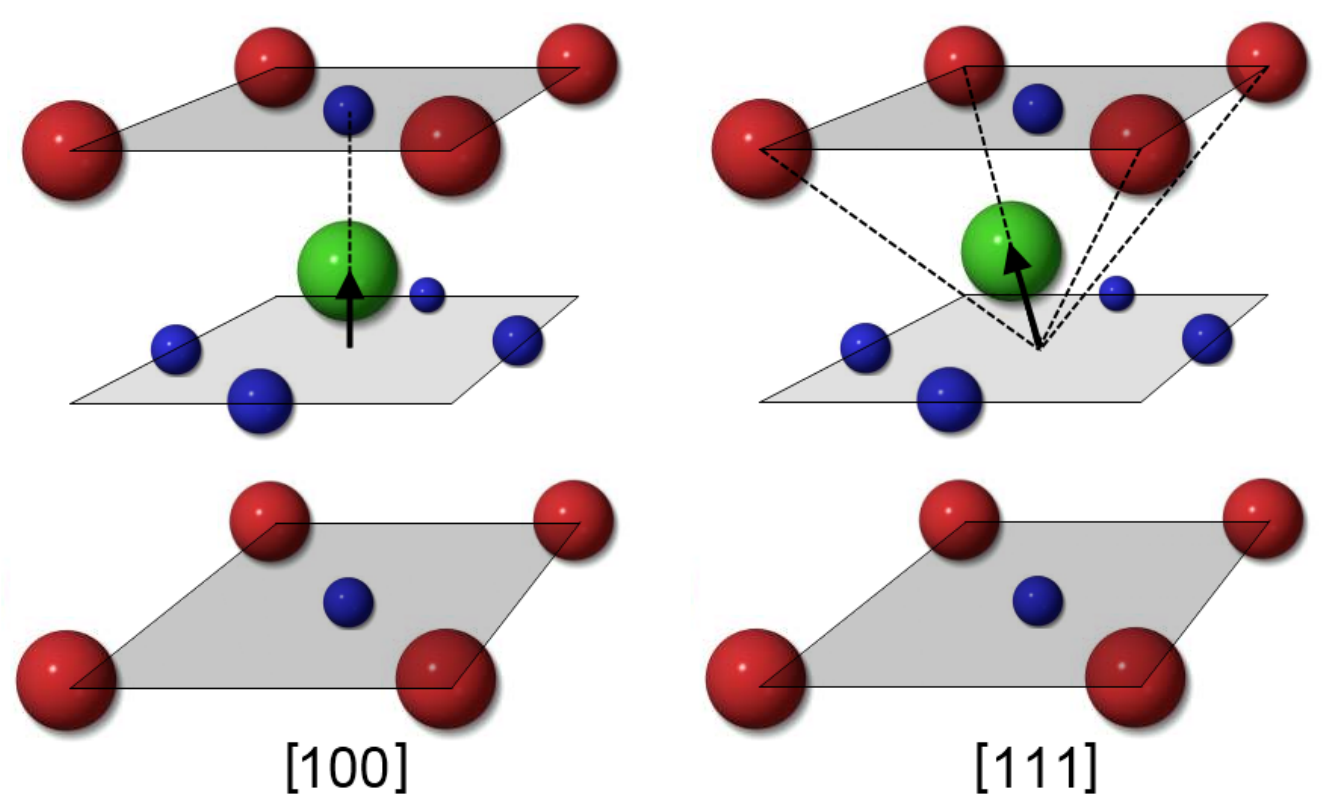

Figure 1.4: Two displacements of the b-site cation in tetragonal perovskites that can lead to ferroelectricity.

[111] directions that were closest to the direction of poling (and which therefore had the lowest potential energy). The net polarisation would be smaller than that of a [111] poled example of such a crystal, as the vector sum of individual dipoles still involves some degree of cancellation. Two different displacements leading to ferroelectricity in perovskites are illustrated in Fig. 1.4.

\subsubsection{Properties of Ferroelectrics}

The signature property of ferroelectrics is of course their electric dipole switchability. But ferroelectrics possess a number of other interesting material features as well.

The ferroelectric properties of materials are most directly observed via poling hysteresis loops - external electric field applied vs measured material polarisation. The key properties found from this experiment are the remnant polarisation at zero 


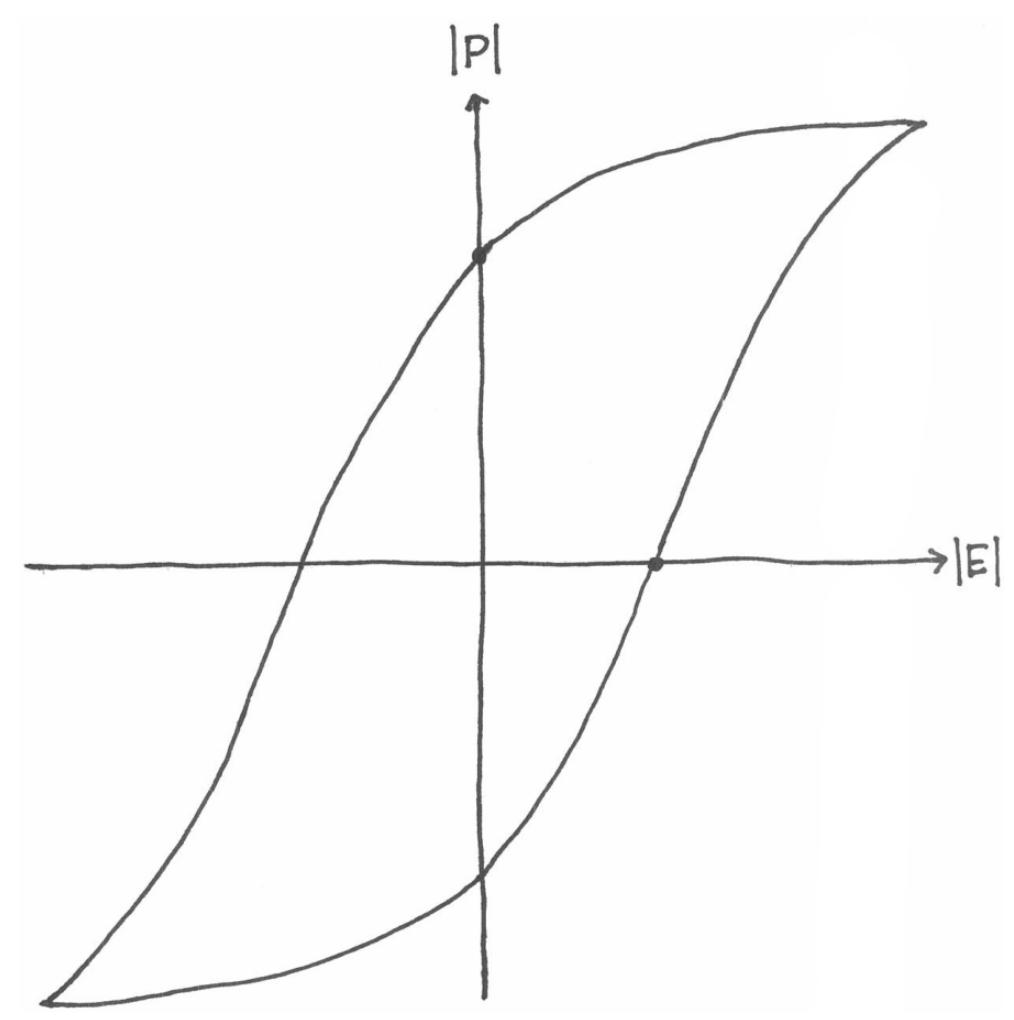

Figure 1.5: A ferroelectric hysteresis loop. /itE is the electric field applied to the ferroelectric, and /itP is the resulting polarisation of the ferroelectric

applied field (y-intercept), and the coercive field necessary to induce switching ( $\mathrm{x}$ intercept). A ferroelectric hysteresis loop is illustrated in Fig. 1.5.

Ferroelectrics are known for their large relative permittivities. This is easy enough to intuitively understand - ferroelectrics have switchable polarisation; they have great capacity to alter their structure to respond to electric fields, and in doing this, the electric field does work on them, effectively shielding the influence of the electric field beyond them. This results in a strong dielectric response. The uncommonly high permittivities of ferroelectrics make them effective dielectric components of capacitors and other devices. The dielectric properties of ferroelectrics are explained in more detail in section 1.1.3 below.

As the ferroelectric effect is so critically dependent on the symmetry of the crystal lattice, we always find in ferroelectrics a strong coupling between mechanical and electric forces. This is easy to conceptualise, as any change to the crystal lattice will necessarily change the dipole moment of the unit cell, causing a change 
in the ferroelectricity. This change can be continuous or it can be abrupt. All ferroelectrics also display piezoelectricity and pyroelectricity as a consequence of this strong coupling with the crystal lattice.

Pyroelectricity is the ability of a material to produce a voltage in response to a change in temperature - the change in temperature alters the crystal lattice, by thermal expansion or other methods, and this changes the polarisation vector (remember that ferroelectrics always have polar crystal lattices). The net effect is a potential across the crystal. This potential can be easily neutralised by movement of charge however, making the effect temporary. Pyroelectricity has limited technological applications, mostly being used in infrared sensors.

Piezoelectricity is the coupling of electrical and mechanical forces in a material. Pressure, vibrations, or other kinds of physical stresses applied to the material will generate an electric charge. The inverse is also true - any voltages applied to the material will result in expansions or contractions of the physical crystal. Piezoelectricity is illustrated in a cartoon in Fig. 1.6. Unlike pyroelectricity, piezoelectricity is an important an widely used material property of ferroelectrics. Piezoelectricity is quantified by the piezoelectric tensor, $d$. This quantity is a tensor, and it acts between electric and mechanical factors. It describes via coupled equations both the contribution to the electric displacement field, $\mathbf{D}$ of an applied stress $\mathbf{T}$, and (via its transpose) the contribution to strain, $\mathbf{S}$ of an applied electric field, E:

$$
\begin{gathered}
\mathbf{D}=d \mathbf{T}+\epsilon \mathbf{E} \\
\mathbf{S}=s \mathbf{T}+d^{T} \mathbf{E}
\end{gathered}
$$

where $\epsilon$ is the matrix for permittivity at constant stress, and $s$ is the matrix for compliance at constant electric field. So in general, $d$ is a third order tensor. There are many elements of this tensor, and although a high coefficient in any of them could be exploited in technology, usually the metric used to judge piezoelectric materials is the component $d_{33}$ which measures the field evolved in the z direction 
from an applied normal (ie - not shear) stress applied in the same direction. The units of $d_{i j}$ are $\mathrm{mV}^{-1}$ or $\mathrm{CN}^{-1}$, with higher values representing higher polarisations developing from smaller applied stresses. Higher values of $d_{i j}$ also mean higher mechanical deformations evolved from smaller electric fields, so a high value is best for both types of piezoelectric application.

\subsubsection{Ferroelectric Phase Transitions}

As ferromagnetic materials are raised in temperature, they eventually reach a point at which thermal energy is sufficient to randomise the direction of the individual magnetic dipoles, creating a disordered array with no net magnetisation. This effect is a phase transition and the temperature at which is occurs is called the Curie temperature, $\mathrm{T}_{C}$. This thermal destruction of order can occur in ferroelectric materials also, and the temperature is likewise called the ferroelectric Curie temperature, $\mathrm{T}_{C}$. In ferroelectrics, in contrast to ferromagnetics, it is also common for a material to lose its ferroelectricity on heating due to a structural phase transition into a centrosymmetric structure (ie, it is not the ordered directions of the dipoles that has been destroyed, but rather the existence of the dipoles themselves). In this case, the temperature point at which ferroelectricity is lost is still called the Curie temperature. The former kind of loss of ferroelectricity is known as a displacive phase transition, the latter as an order-disorder phase transition.

Ferroelectrics near to the Curie temperature exhibit an extraordinary increase in their piezoelectric coefficients[4] - smaller stresses cause larger polarisations. Many devices benefit from high piezoelectric coefficients - sensors become more sensitive, for example; spark elements can more easily create the voltages required to form a spark, and energy harvesters increase in efficiency. Hand in hand with the increase in piezoelectric coefficients comes a further extraordinary increase in the dielectric permittivity, both of them experiencing resonant-type peaks at the transition temperature. This increase in the piezoelectric and dielectric coefficients is driven by the flattening of the energy landscape near to the transitions - the potential energy levels of different polarisations and between different crystal geometries become very similar, and the barriers between them very shallow so they require only small stresses to substantially alter their crystal structures. In other 


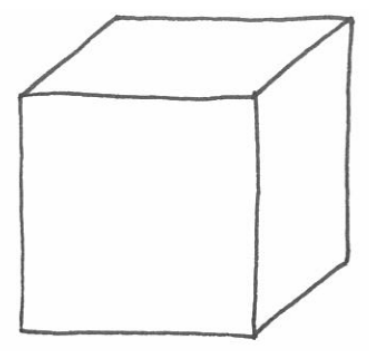

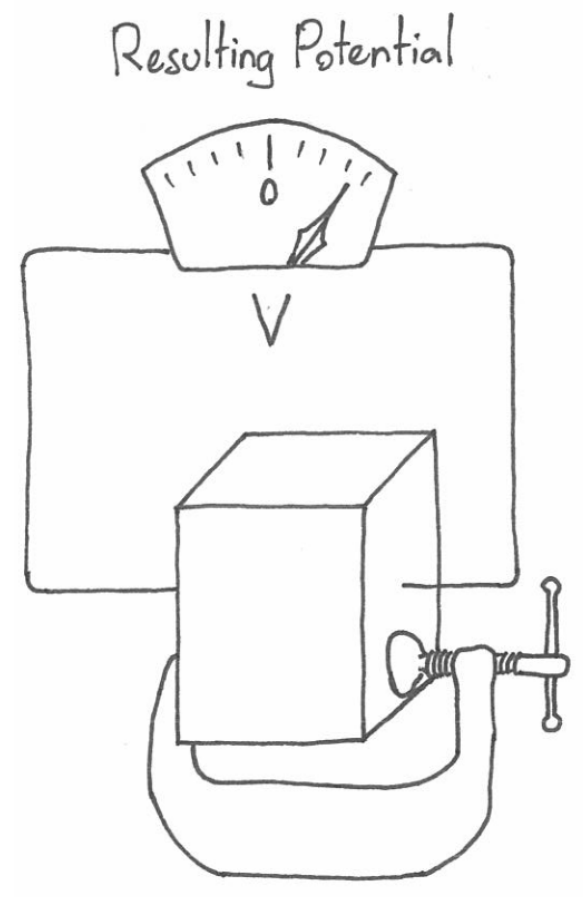

Applied Stress
Applied Potential

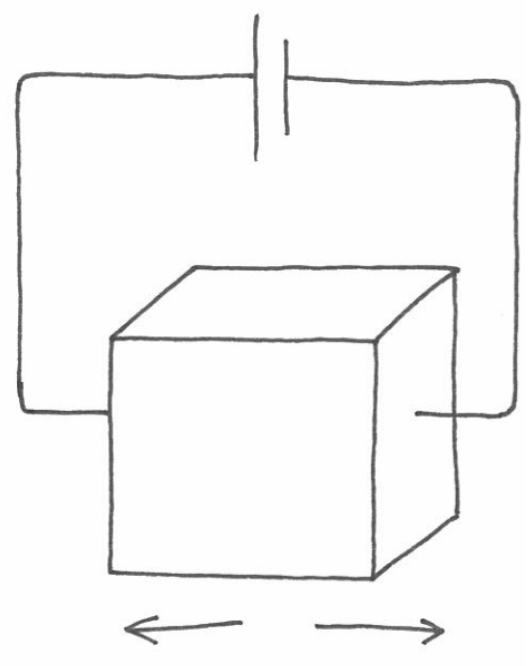

Resulting Strain

Figure 1.6: Cartoon depiction of piezoelectricity. Applied stress results in a potential across the material, and conversely applied potential results in strain. 

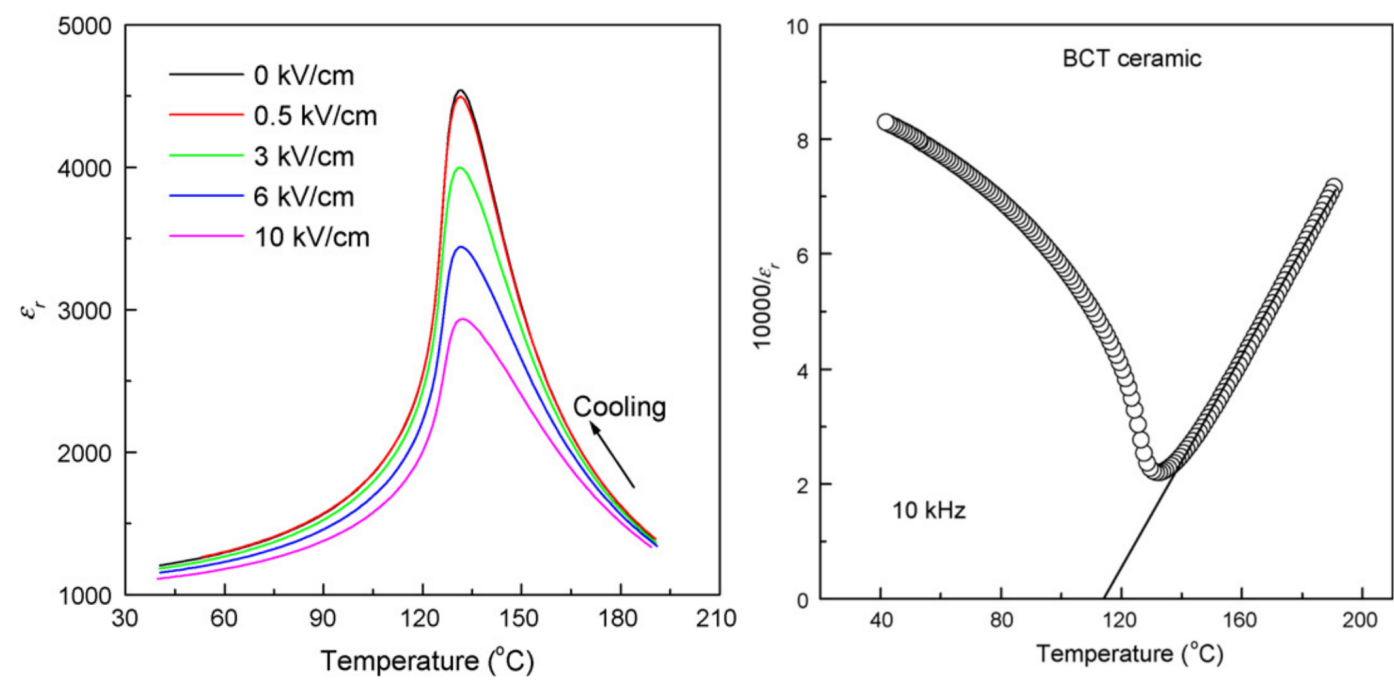

Figure 1.7: The distinctive 'Curie Weiss' behaviour of the dielectric permittivity of a ferroelectric material. The peak in the permittivity-temperature graph corresponds to the ferroelectric phase transition temperature. Both permittivity and inverse permittivity are shown (figure reproduced with permission from [5]).

words, switching polarisation from one direction to another becomes very easy.

The behaviour of the dielectric permittivity of a ferroelectric material, $\left(\mathrm{Ba}_{0.9} \mathrm{Ca}_{0.1}\right) \mathrm{TiO}_{3}$ is shown in Fig. 1.7, reproduced from [5]. This behaviour is typical of ferroelectric materials, and is described by the Curie-Weiss equation:

$$
\frac{1}{\epsilon_{r}}=\frac{T-T_{\mathrm{C}}}{C}, T>T_{\mathrm{C}}
$$

where $\epsilon_{r}$ is the relative dielectric permittivity, $T$ is the temperature of the material and $T_{\mathrm{C}}$ is the Curie temperature of the material. $C$ is some constant parameter. The relative permittivity rises proportional to $1 /\left(T-T_{\mathrm{C}}\right)$ above the Curie temperature, peaks at the Curie temperature and then falls again afterwards.

Among the phase transitions of ferroelectric perovskites, transitions between tetragonal and rhombohedral structures are of particular interest. In PZT, the most commonly used ferroelectric, a transition of this type occurs as a function of composition at around $48 \% \mathrm{Zr}$ content (depending slightly on temperature). The boundary is known as a morphotropic phase boundary (MPB). This MPB separates a rhombohedral phase on the Zr-rich side from a tetragonal phase on 


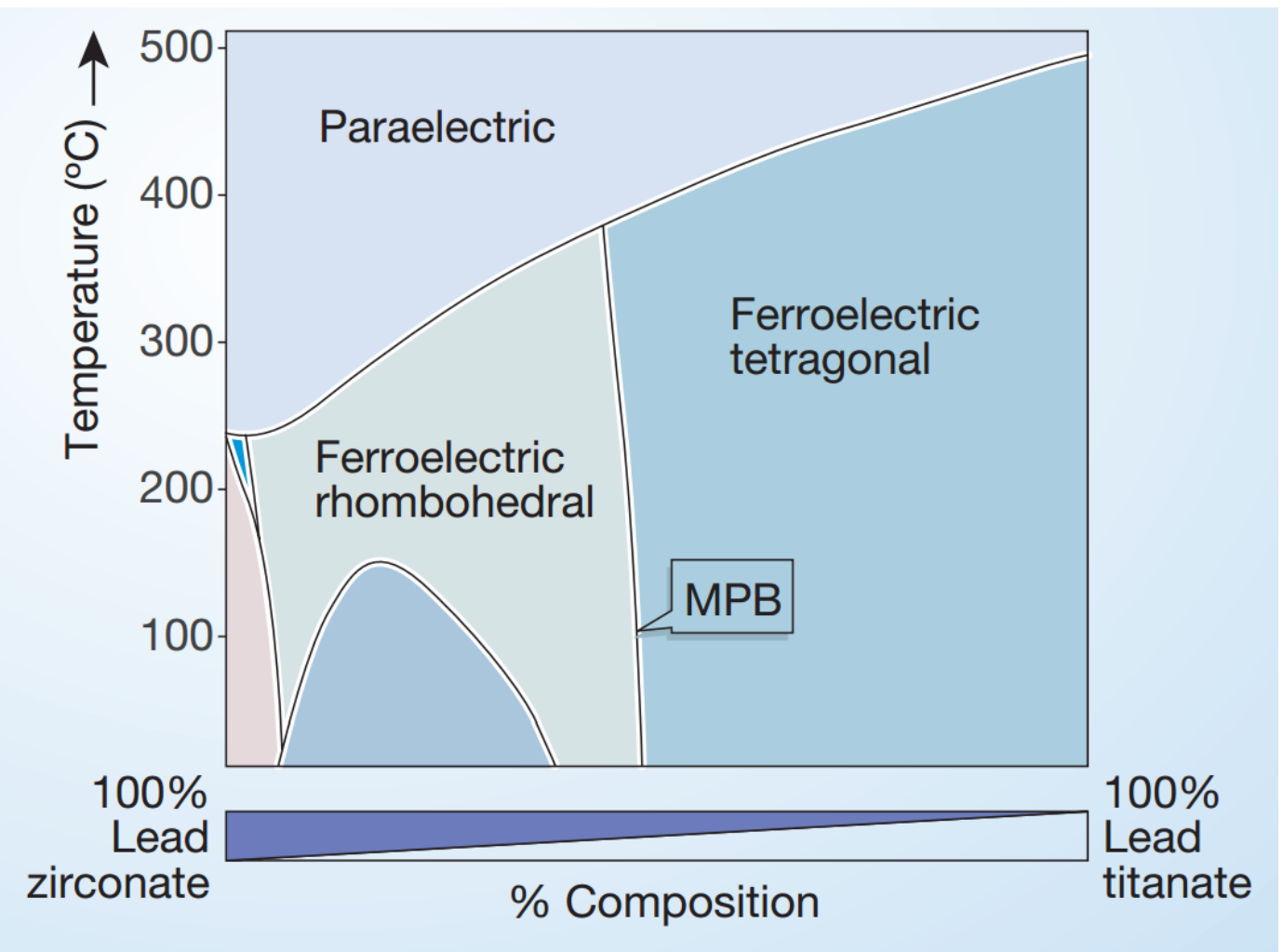

Figure 1.8: Phase diagram of lead zirconate titanate, emphasizing the morphotropic phase boundary (Reprinted by permission from Macmillan Publishers Ltd: Nature[9], copyright 2004).

the Ti-rich side, and is thought to be mediated by a monoclinic phase between the two[6]. Because of the rich variety of different unit cell polarisations all available to the material on the cusp of this transition (6 [001] directions, 8 [111] directions plus any other accessible to the intermediate monoclinic phase), polarisation switching becomes very easy. The unusually flat free energy profile results in tremendously enhanced piezoelectric coefficients in PZT of this composition[7][8]. Another added advantage of MPBs of this type is that they are relatively flat with regard to temperature. This means that any devices made with PZT of compositions near to the MPB to take advantage of the enhanced properties will remain functional over a wide range of temperatures. MPBs are commonly sought out in other materials as an indicator of strong ferroelectric properties. Fig. 1.8 is reproduced from [9]. It is a phase diagram of PZT, pointing out the MPB. 


\subsubsection{Ferroelectrics in Technology}

In technology, ferroelectric materials are currently used in: spark elements, such as cigarette lighters or the lighter for gas stoves (taking advantage of the piezoelectric effect); motion, sound and vibration sensors and actuators including types of microphone and car fuel injection systems (also taking advantage of the piezoelectric effect); and also in miniaturised radio-frequency electronics. In this application, it is the high dielectric permittivity rather than the piezoelectricity or ferroelectricity that is important - to make a capacitor of similar capacitance at a smaller size, one requires a dielectric material of higher permittivity. Ferroelectrics have also been trialled in lab or field situations for use in energy harvesting applications, turning impacts such as footfalls into electricity; and in non-volatile memory, storing bits as the polarisation state of a ferroelectric. There are also suggestions in literature that ferroelectrics may play an important role in micro electrical-mechanical machines in the future, if these become significant products. They could be used to convert electrical signals into movement in such devices. Of the most current economic importance are their use as transducers in all kinds of electronic devices to sense and create motion[10].

As mentioned earlier, most of the ferroelectrics used in technology are made from some composition of PZT, with modifications depending on what kind of frequency, power, stability, mechanical properties etc. are required from it. And while they are generally safe during the functional life of the product, both the manufacture and the disposal of the product containing PZT becomes a health and safety issue due to the lead content. There are also some concerns about the use of lead in medical electronics. Lead is, of course, a highly toxic chemical to the human body, even in small amounts. Lead-based damage to the nervous system is particularly dangerous, as it is largely irreversible.[11]

The health risks associated with lead in piezoelectrics has prompted lawmakers in many countries to order a gradual phase out of lead-containing materials in all electronic devices. In the European union, the 'Restriction of the Use of Certain Hazardous Substances' act 2006 and the 'Waste from Electrical and Electronic Equipment' act of 2005 restrict the manufacture of lead containing products [12]. However, due to the unsuitability of currently available lead-free piezoelectrics, 
an exemption is given to PZT in this legislation.This exemption is given in the expectation of a suitable replacement being developed to eventually replace it. This has driven commercial, and commercially focused government research groups to devote research to finding a functional lead-free piezoelectric, as there is a guaranteed market for such a new product. China, Korea, and some states of the USA have also introduced legislation against lead-containing piezoelectrics.

A body of research has been done on trying to find other perovskite systems that display piezoelectric properties of a similar strength to PZT, with some successes. The $\mathrm{Na}_{x} \mathrm{Bi}_{1-x} \mathrm{TiO}_{3}-\mathrm{BaTiO}_{3}$ and $\mathrm{K}_{x} \mathrm{Na}_{1-x} \mathrm{NbO}_{3}$ systems both show some promise, although neither is as good as PZT. Lead has shown itself to be conducive to stronger piezoelectric properties than other elements. No material with stronger piezoelectric properties than the lead based systems has yet been found, and it is reasonable to expect that continuing to search for replacements on a purely chemical basis may never yield the desired results. Computations based on synchrotron x-ray diffraction data show an unusually strong binding between the lead ion and the oxygen ions in lead titanate crystals, which is thought to be in part responsible for the high piezoelectric properties shown in PZT[13].

Fortunately, chemical substitution is not the only avenue of exploration open to us. It has long been known that nanoscale materials behave differently to the bulk due to increased surface energy, quantisation of energy levels and symmetry breaking. Research into the properties of ferroelectrics and piezoelectrics at the nanoscale are a promising direction for lead-free material discovery and enhancement. Lattice strain is also an interesting avenue of research, crystals that are externally constrained in their lattice constants have different lattice dynamics and properties than their fully relaxed bulk forms. In this work, both nanoscale modifications and lattice strain are tested for their effects on ferroelectric properties of materials.

\subsection{Raman Spectroscopy}

The technique of Raman spectroscopy is based on the Raman scattering, a type of inelastic scattering of light from materials. This type of scattering was discovered 
1.2. Raman Spectroscopy

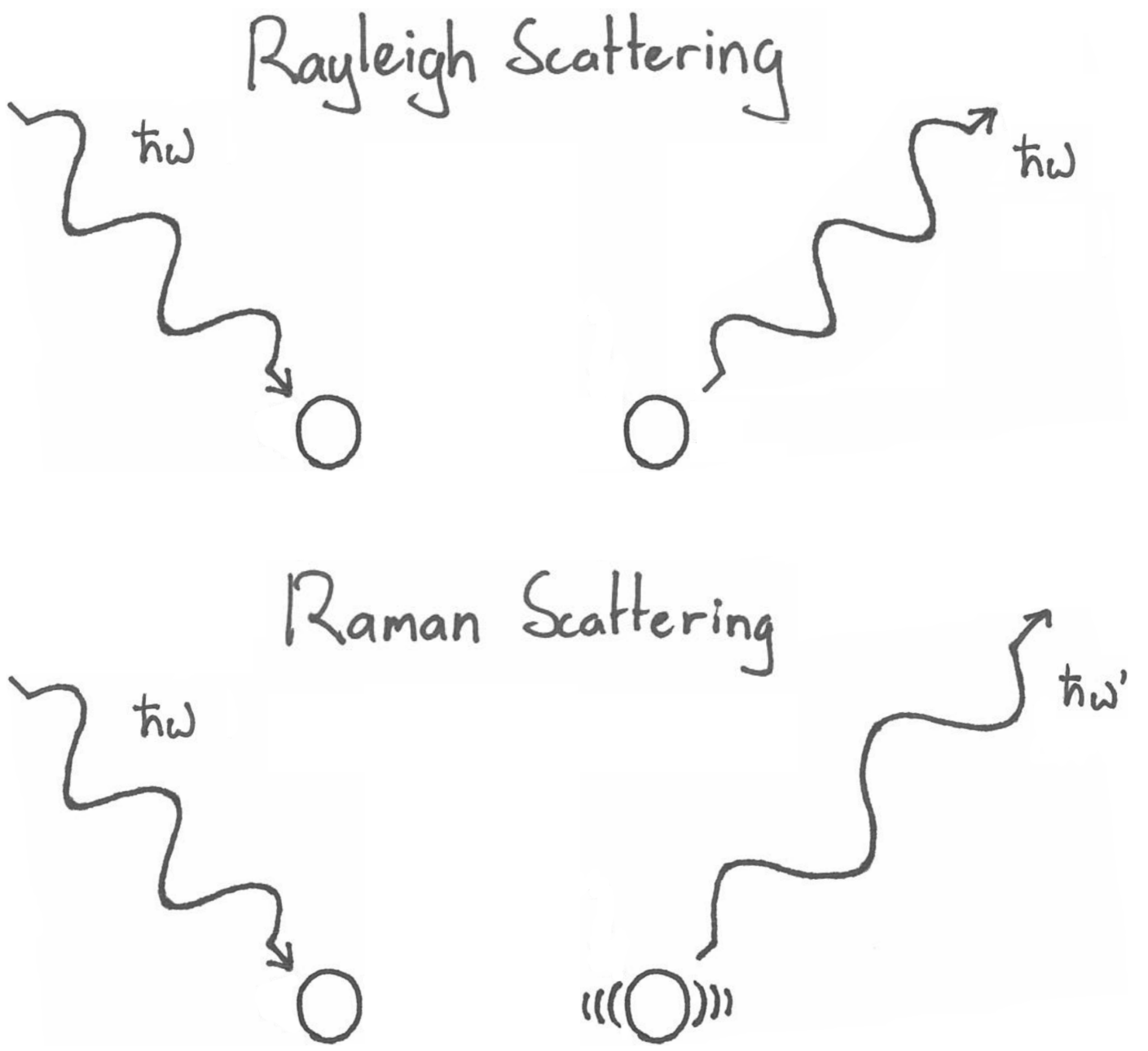

Figure 1.9: A Roman scattering event: incoming light interacts with a vibrational mode of the molecule, and is scattered with altered energy (frequency). Compare this with Rayleigh scattering, where scattered energy is the same as incoming.

by, and named after C. V. Ramon in 1928. When light is incident on a material, several things may happen. Most commonly, the light may be reflected, it may be transmitted through the material, or it may be absorbed by the material (typically by an electron promotion). Rarely, the light will be scattered not through the normal Rayleigh process but by interaction with a third entity, a normal vibrational mode in a molecule (or crystal). This is Roman scattering, and it is approximately $10^{7}$ weaker than Rayleigh scattering. A Roman scattering event is illustrated in Fig. 1.9.

16 


\subsubsection{What is a phonon?}

Atoms in molecules or crystals cannot vibrate independently. They are linked by bonds to other atoms, and as such only a finite number of vibrational patterns can be stable for any given structure; all others will decay rapidly with time. The stable vibrational patterns are known as the normal modes of the molecule or crystal. The normal modes can be characterised by a set of displacement vectors detailing how each atom moves in that vibration. Each normal mode has a characteristic frequency (which is representative of its energy), wavevector (which is representative of its momentum), and symmetry associated with it. In crystals, periodic boundary conditions apply, further restricting the allowed vibrations. Normal modes are oscillators, and as such their energy is quantised as $E=(n+1 / 2) \hbar \omega$. It is the energy quanta of these normal mode oscillations that we call phonons. The name is chosen to be analogous to photons, as the two concepts are very similar photons count the excitation number of normal electromagnetic radiation modes inside blackbody cavities, and phonons count the excitation number $(n)$ of normal vibrational modes in molecules or crystals. Saying that $n$ phonons of a given wavevector, frequency and symmetry are present in a crystal is therefore another way of saying that the normal oscillation mode in the crystal corresponding to that wavevector, frequency and symmetry is in the $n^{\text {th }}$ excited state. Phonons do not have a well defined spatial extent. They persist in a delocalised state in the crystal, over the area for which the periodic boundary and symmetry conditions hold. In Raman scattering, it is these vibrational normal modes that scatter the incident light. A phonon is illustrated in figure 1.10 .

\subsubsection{Raman scattering}

In solids, first-order Raman scattering involves (in addition to the incident and scattered light) either the creation of a new phonon, or the absorption of an existing phonon in the material upon which the light is incident. The former is called Stokes scattering, and the scattered light has a lower frequency(energy) than the incident. The latter is called anti-Stokes scattering, and the scattered light has a higher frequency than the incident. It is also possible to have multiple-phonon scattering, where the incident light interacts with more than one phonon. This is 


\section{This is a normal vibrational mode:}

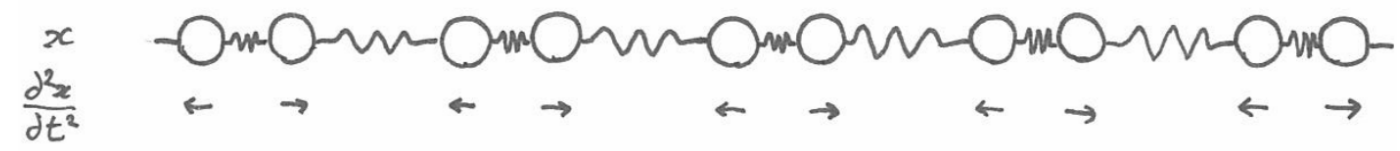

The amount of energy in the mode is quantised in phonons

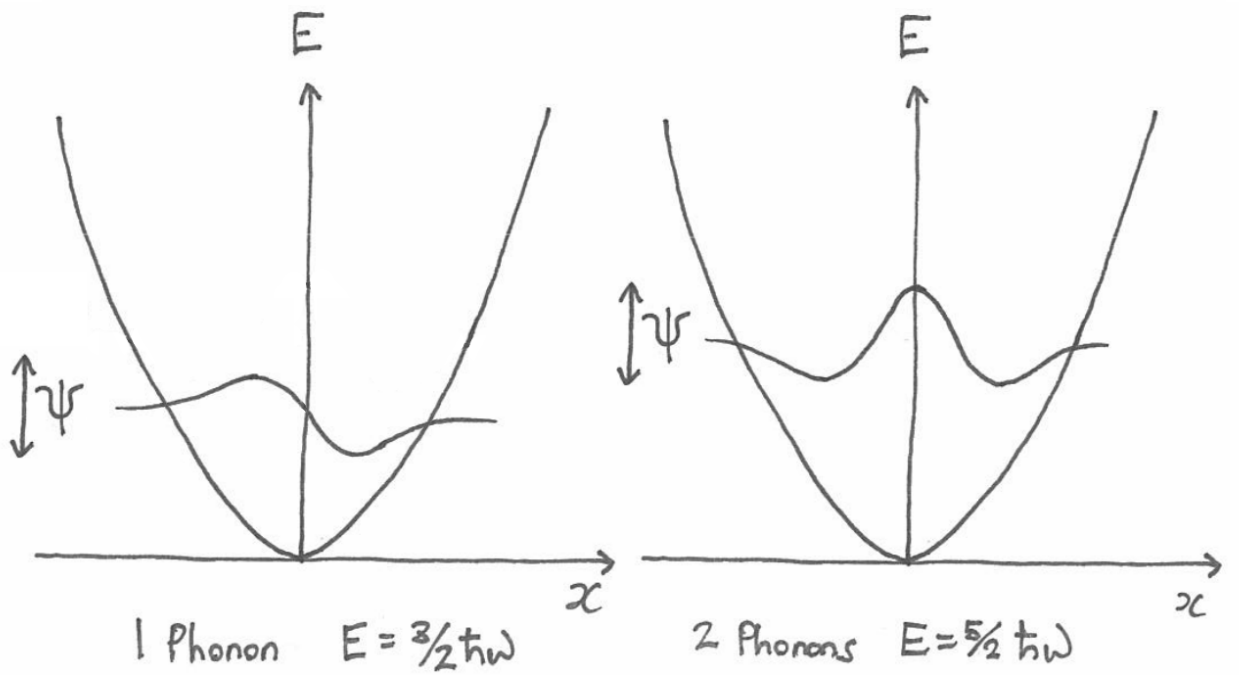

Figure 1.10: Phonons. Shown above is a normal vibrational mode of a 1d crystal. Below - two different energy levels of this vibration, wavefunction vs displacement. Left - the first excited state left (one phonon) and right - the second excited state (two phonons). 


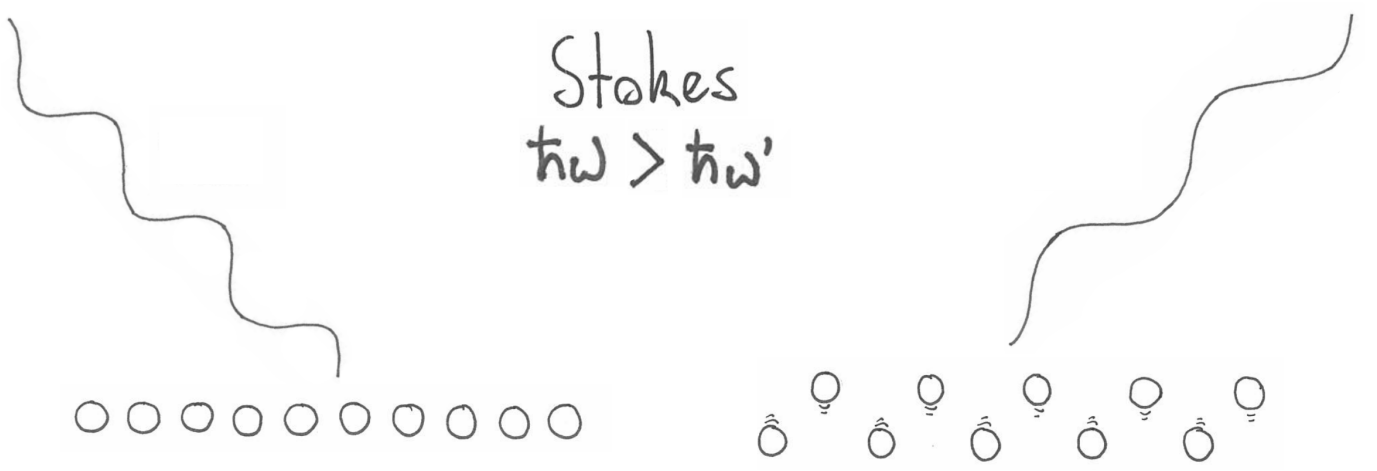

Figure 1.11: In Stokes scattering a vibrational mode in the material gains an energy level (phonon) and the scattered light has lower frequency (energy).

known as second (or higher) order Raman scattering. It is weaker and broader in frequency than first order scattering. In higher order Raman, it is possible for both creation of phonons and annihilation of phonons to occur in the same scattering event. Figs. 1.11 and 1.12 illustrate Stokes and anti-Stokes scattering respectively.

There is a strong difference in intensity between Stokes and anti-Stokes Raman scattering. For Stokes scattering to occur, there needs to be an occupied lowenergy vibrational state in the material the incident light strikes, and a vacant high-energy vibrational state for it to move into. The Stokes scattering probability is accordingly proportional to the number of low-energy vibrational states that are occupied in the material, and the number of high-energy vibrational states that are vacant. Conversely, the anti-Stokes scattering probability is proportional to the number of vacant low-energy states, and of occupied high-energy states. Typically at room temperature, the number of occupied low-energy states in materials is far greater than the number of occupied high-energy states, and so Stokes scattering is much more intense than anti-Stokes scattering. At elevated temperatures, antiStokes scattering can be more intense. In fact, from the ratio of Stokes to antiStokes scattering in a sample, the temperature of the sample can be deduced.

Unlike fluorescence, Raman scattering is an instantaneous event. The system does not go through an intermediate excited state between the incident and scattered light. It therefore preserves momentum and polarisation, and does not require a physical state at energy $E+\hbar \omega$ (the incoming light energy) in order to 


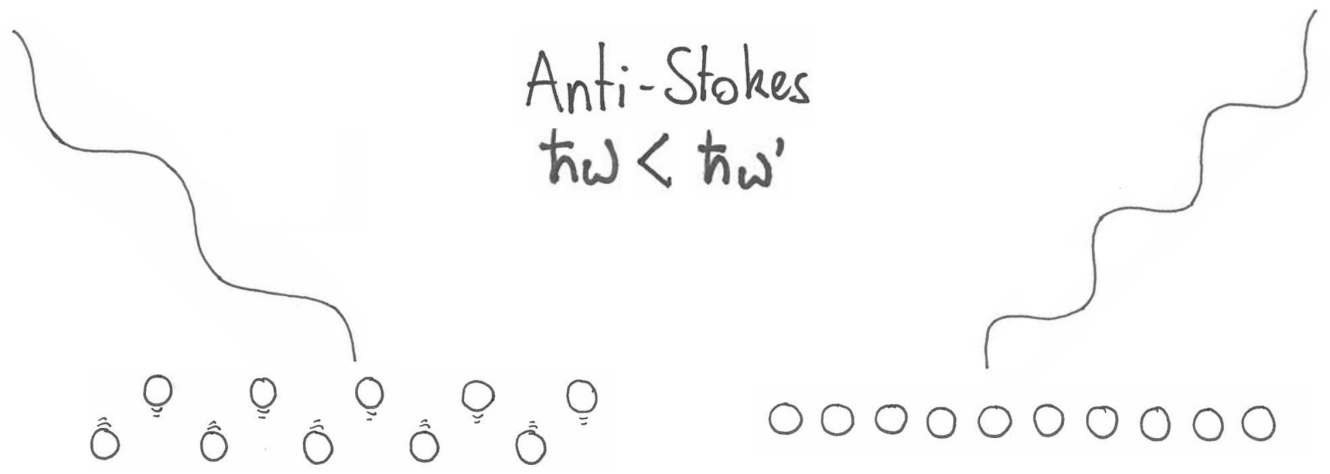

Figure 1.12: In anti-Stokes scattering a vibrational mode in the material loses an energy level (phonon) and the scattered light has higher frequency (energy).

occur. ${ }^{1}$ The presence of a physical electron transition in a material at an energy near to the excitation light's energy $\hbar \omega$ however, does increase the scattering cross section. This is known as resonant Raman spectroscopy. The phenomenon cannot be described in the classical picture, but comes out naturally from the full quantum and semi-classical mathematical formulae (in the semi-classical treatement, the electric field is continuous rather than quantised). Sticking to the tunneling analogy, the electron state lowers the barrier height between the incoming and Raman scattered light, thus increasing the tunneling probability (in tunneling one can lower the barrier sufficiently that the wave could find itself in the barrier region, and here the metaphor breaks down because resonant Raman spectroscopy can still never cause the material to exist in the 'virtual state').

The full mathematical treatment of resonant Raman scattering is beyond the scope of this thesis, but for a fuller understanding it is useful to examine the semiclassical equation for a given element of the Raman polarisability tensor. Such a treatment is found in appendix B of this thesis.

Not all phonons are Raman active. In order for a Raman scattering event to occur, there are certain symmetry conditions that must be fulfilled. First, Raman

\footnotetext{
${ }^{1}$ Many descriptions of Raman scattering explain it in terms of an intermediate 'virtual state,' but I personally dislike this visualisation - it was a source of confusion for me. The virtual state is merely a mathematical construction. The system spends no time in this state; one can never observe the system in it. It makes no more sense than to say that a particle in a double well physically passes through the barrier - it does not. Its wavefunction on either side of the barrier penetrates through it into the other allowed state and it seamlessly switches from one state to the other.
} 
scattering is related to the polarisability of molecules and materials. The polarisability relates the expressed polarisation of an object to the applied electric field, in the form of a second rank tensor. The Raman cross section, $\mathbf{R}$, is proportional to the first order derivative of the polarisability tensor with respect to the phonon displacements,

$$
\mathbf{R} \propto \partial \chi / \partial \mathbf{G}
$$

where $\chi$ is the polarisability tensor and $\mathbf{G}$ is a tensor describing the phonon displacements. If the partial derivative is zero for a mode, then it does not Raman scatter light. The displacements of the phonon mode must therefore alter the polarisability in order to undergo Raman scattering for the phonon in order for it to show Raman scattering. Secondly, the intensity of Raman scattered light is proportional to the inner product of the unit polarisation vectors of the incoming and outgoing scattered light:

$$
I \propto\left(e_{i} \cdot \mathbf{R} \cdot e_{s}\right)
$$

where $e_{i}$ and $e_{s}$ are the unit polarisation vectors discussed above. Thus, vibrational modes described by $\mathbf{G}$ may be visible in some polarisations of Raman measurement, and invisible in others.

So, some phonons are completely Raman silent, and others may only be accessed with particular polarisations of incoming and scattered light. What can be seen in Raman is, however, more than enough to make it an effective technique for chemical fingerprinting and (with the aid of computational models) structural determination. Experimentally, polarisation rotators are used to control the polarisation of incident laser light, and polarisors are used to remove from the scattered light all polarisations except that which is to be measured.

Because of the significant effect of polarisation on Raman measurements, there have been several formalisms developed for describing the polarisation condition of measurements. In this thesis, the following convention is used: Labels have the 
form $i(j k) l$ where $i, j, k, l$ are each one of $x, y$, or $z$ geometrical diretions. Typically, $z$ is chosen to be parallel to the [001] axis of crystals, and $x$ and $y$ are perpendicular to $z$ and to each other in the usual right handed manner. In all instances in this thesis, we denote $x, y$, and $z$ as [110], [110] and [001] respectively. The first letter, $i$, describes the direction of motion of the light incident on the sample, and the last letter, $l$, describes the direction of motion of the scattered light that is collected by the spectrometer. $j$ is the direction of the electric field vector of the incoming light, and likewise $k$ is the direction of the electric field vector of the scattered light that is collected by the spectrometer. So, for example, the label $x(y z)-x$ denotes an exciting laser beam along the [110] axis with electric field vector pointing along [1]̄0], and scattered light travelling back along the [110] axis with electric field vector pointing along [001]. So - in other words it is a back-scattering geometry which measures the Raman scattering polarised in-plane perpendicularly to the incident beam.

The symmetry conditions of Raman scattering are different to the symmetry conditions for infrared absorption, which is another technique used to probe vibrational modes. Because they are different, the two methods can complement each other, revealing more about the material by the use of both techniques. It is however entirely possible for a phonon mode to be active in both Raman and IR, and likewise for it to be inaccessible to both.

\subsubsection{Spectroscopic method}

The most easily observable result of Raman scattering, then, is scattered light with an altered frequency. If one excites a material with light of a single wavelength, then one can collect the scattered light, then disperse it by wavelength and measure the intensity of the dispersed light at each angle. Once the light of the excitation frequency is removed, the resulting spectrum shows the intensity of Raman scattered light, resolved by the frequency of the wave. The energy shift of the scattered light is numerically equal to the energy of phonons that are present in the material (and Raman active). Therefore, a Raman spectrum shows the energy of phonons in the measured material. A Raman spectrum of silicon is shown in Fig. 1.13. You can see the Rayleigh scattered light, the Stokes scattering from 


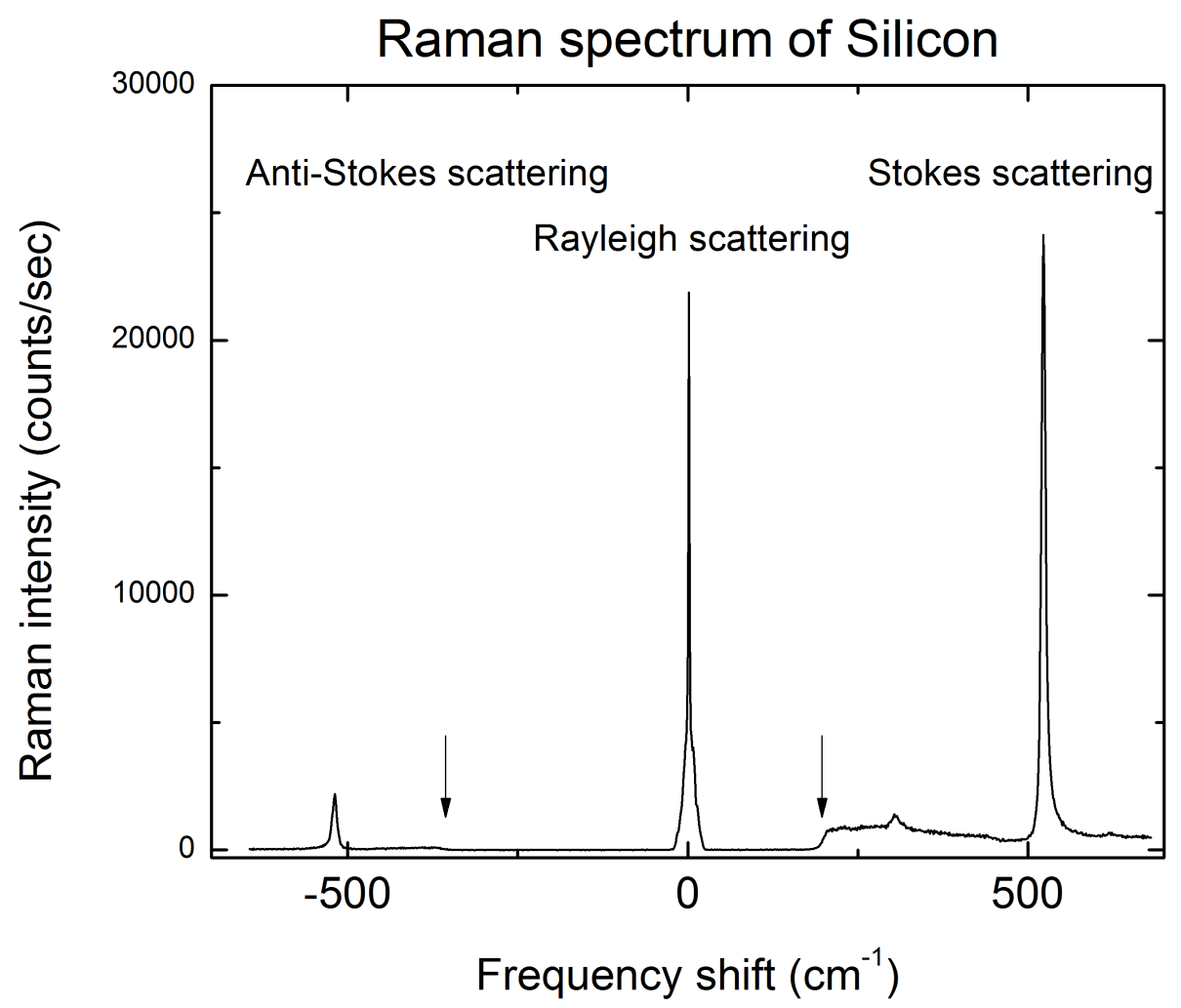

Figure 1.13: Raman spectrum of silicon. The Rayleigh scattered light can be seen in the centre, the Stokes scattered light on the right and the anti-Stokes scattered light on the left. Note that this means that light of higher frequency is on the left of the spectrum, not the right - ie, Raman shift is equal to the negative of the change in frequency. This is the usual way that Raman spectra are displayed. The arrows in the spectrum show the cut off points provided by the notch filter. The Rayleigh scattered light is in fact much stronger than is shown here - it is being heavily attenuated.

silicon modes and the anti-Stokes scattering from silicon modes Both first and second order scattering can be seen.

The technique can be augmented in various ways to increase its functionality or its power. For example, one can finely control the polarization of the incoming light and measure the polarisation of the scattered light to find out the orientation of single crystal samples and the symmetry of the phonon modes. Confocal microscopy is also compatible with Raman spectroscopy, allowing Raman to probe very small regions and build up 2D or even 3D images of chemical information on length scales of 1 micron. It is possible to build temperature- and pressurecontrolled cells in which to carry out experiments also - one need only to have an 
optically transparent window. This allows temperature and pressure dependent Raman spectra to be measured which is useful in studying phase transitions. One can also increase the scattering cross-section of Raman spectroscopy by using resonant Raman spectroscopy or surface enhanced Raman spectroscopy techniques of which more later.

The technique is often used as a fingerprinting method for chemical identification, along with IR spectroscopy. But for our research, we are most interested in it because it give us information about the structure of crystals. Shifts in parameters such as unit cell volume, c/a ratio, defect density etc. can result in changes to the Raman shift, strength and width of phonon peaks, and changes in crystal symmetry can cause more drastic changes, making new peaks appear, disappear, split or merge. The Raman spectrum of a crystal can therefore be used as a proxy for direct structural measurements (such as XRD), with theoretical models linking specific changes in vibrational states to changes in structure. It is these changes that will track as we apply external stresses to the ferroelectric materials we are studying.

\subsubsection{Soft mode spectroscopy}

One particular feature visible in Raman spectra that is notable around structural phase transitions in crystals is a phenomenon known as a soft mode. Excellent descriptions of soft modes and of their experimental uses are given in [14] and[15]. Soft modes are a particular kind of normal vibrational mode in crystals, whose (oscillating) set of displacement vectors are the same as those static displacement vectors that lead to a phase transition. In other words, if one were to take the current crystal structure, and impose upon that cell the displacements that describe the soft mode vibration, you would end up with the new structure. This is illustrated for a cubic to tetragonal phase transition in a perovskite in Fig. 1.14. At the phase transition temperature, this is in fact exactly what happens. As T tends towards $\mathrm{T}_{C}$, the frequency of this vibration collapses towards zero and the crystal lattice is left in the distorted state.

Looking at this from an energy perspective, as the phase transition is approached, the difference in potential energy of one structure to the other tends 


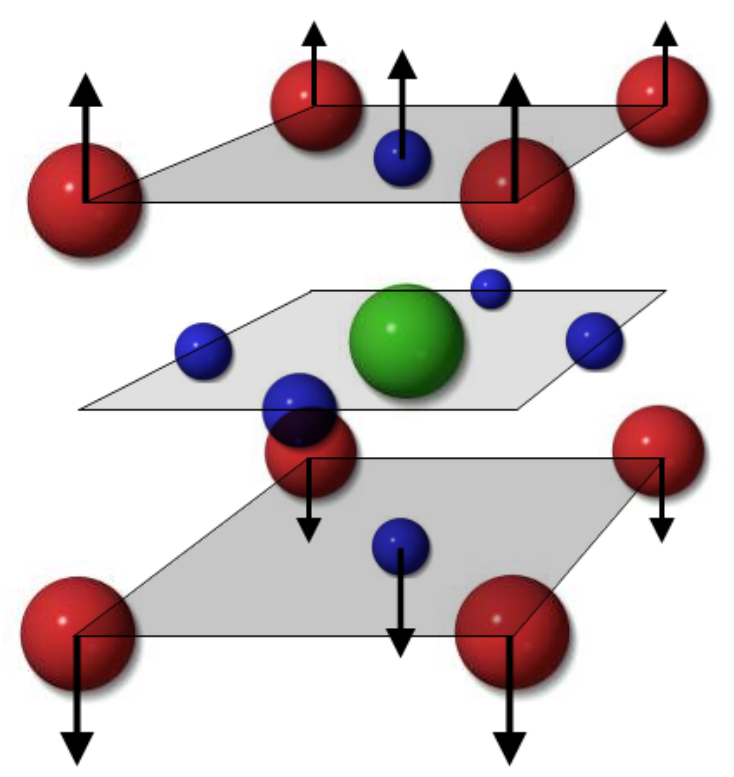

Figure 1.14: Soft phonon mode for the cubic-tetragonal phase transition in a perovskite. The atomic displacement vectors for the vibration match those for the transition. 


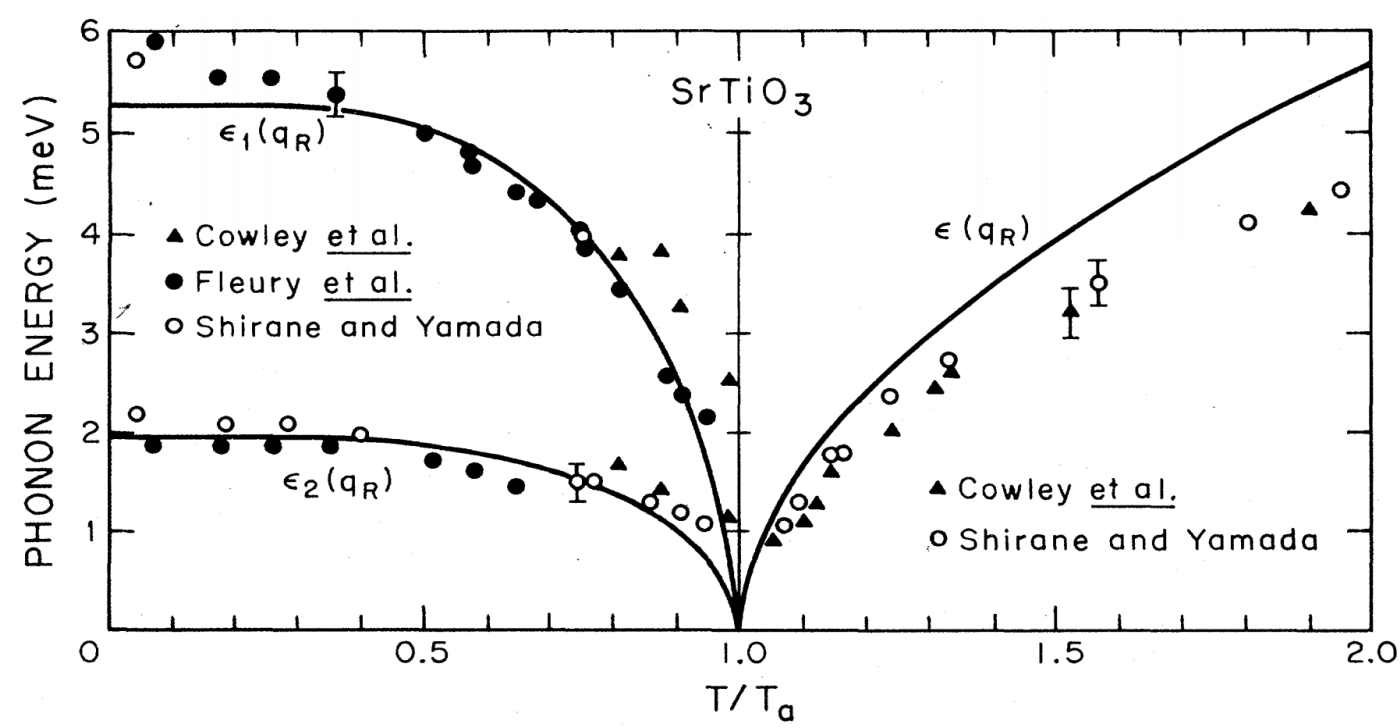

Figure 1.15: Real data showing the frequency of the anti-ferrodistortive soft mode in $\mathrm{SrTiO}_{3}$. The temperature $T_{a}$ in the figure is the temperature of antiferrodistortive phase transition in STO. Note that the frequencies are in this case measured by infrared spectroscopy. Figure reproduced with permission from [14].

towards zero, as does the energy of the barrier between them. We know this must be true, as the most stable structure changes when we cross the phase transition. With the energy barriers becoming so shallow, the restoring force acting on atoms in the soft-mode vibration gets progressively weaker. In an oscillator, this means a slower vibration.

The mechanics and behaviour of soft modes is intimately related to structural phase transitions, and the theory is not trivial. However, it is sufficient for our purposes to note that a mode whose frequency collapses towards zero as temperature is changed is an indication of a phase transition, and it turns out to be a very good one too: its rapid rate of change at the critical point allows a high degree of precision in measuring the transition temperature (or more generally, the critical value of any transition parameter). Fig. 1.15 shows a phase transition in strontium titanate (the cubic to anti-ferrodistortive one), and is reproduced from [14].

One final point that must be made in the Raman measurement of this phenomenon is that it is not always the case that the soft mode is Raman active, and it is not always the case that, should it be Raman active, it is Raman active on both sides of the phase transition. A transition may easily alter the symmetry of the 
crystal, and consequently the soft mode, which may make it symmetry disallowed to Raman scattering.

In this thesis, I have used the technique of Raman spectroscopy as a tool to study various ferroelectric systems, with a view to developing new materials which have better properties, and are free of lead. 


\section{Chapter 2}

\section{Background Information}

This chapter covers important background information about the material systems being studied. For the BNT-BT system and for lead titanate nanowires, only a brief introduction will be required, but for the STO thin films, a much more extensive review of the available literature is necessary. There are numerous reports of Raman studies of STO, but great variation is shown in the Raman spectra. It will take great care to pick through the available literature, and link features in the Raman spectra to structural, chemical and ferroelectric properties of thin films. Complicating the job is the fact that Raman spectra of strained thin films are very difficult to acquire experimentally, so there is a lack of direct comparisons. Instead, spectra of un-strained STO have to be used for the purpose of comparing and interpreting the results that we get. Accordingly, background information on STO will take up the majority of this chapter.

\section{$2.1 \mathrm{Bi}_{0.5} \mathrm{Na}_{0.5} \mathrm{TiO}_{3}-\mathrm{BaTO}_{3}$ system}

Chapter 4 of this thesis covers experiments performed on ceramic samples, both poled and depoled, of the $\mathrm{Bi}_{0.5} \mathrm{Na}_{0.5} \mathrm{TiO}_{3}-\mathrm{BaTO}_{3}$ (BNT-BT) system at various bismuth concentrations.

BNT-BT is one lead-free system that has shown some promising ferroelectric properties. Like PZT, it is a solid solution of two perovskite structured materi- 
als. the materials are sodium bismuth titanate (BNT) with barium titanate (BT). There are many similarities between PZT and BNT-BT. The ions of lead and bismuth are very similar in terms of their size and their number of electrons, including a lone electron pair which is thought to strongly contribute to piezoelectric lattice distortion. However, BNT-BT does not display the same strength of piezoelectric response that PZT does. By comparing PZT to similar materials that do not exhibit such remarkable piezoelectric response, one can gain better understanding of the sources of the response. This understanding can then be applied when searching for candidates for new piezoelectric materials, or when engineering optimal properties in existing ones. This provides motivation for the study of the BNT-BT material system.

BNT-BT has chemical formula $\left(\mathrm{Na}_{0.5} \mathrm{Bi}_{0.5}\right)_{1-x} \mathrm{Ba}_{x} \mathrm{TiO}_{3}$. The $0 \%$ BT endmember has $50 \%$ of a-sites occupied by $\mathrm{Na}$ ions, and $50 \%$ occupied by $\mathrm{Bi}$. The b-sites are all occupied with $\mathrm{Ti}$ ions. The addition of BT to the solid solution replaces $\mathrm{Na}$ and $\mathrm{Bi}$ with $\mathrm{Ba}$ ions while preserving the $1: 1$ ratio of $\mathrm{Na}$ to $\mathrm{Bi}$. The material fabrication, and the phase transitions measured by bulk electrical measurements were reported by Takenaka et al. in 1991[16], and this represented an effort to create a material with a MPB by mixing a rhombohedral ferroelectric with a tetragonal one. BNT-BT is cubic at high temperatures. At low temperatures and $x<5 \%$ the material is rhombohedral and ferroelectric. At low temperatures and $x>6 \%$ the material is tetragonal and ferroelectric. Between the two low temperature phases, somewhere between $5 \%$ and $6 \%$ barium content there is indeed a morphotropic phase boundary, which is similar to what is observed in PZT. Takenaka also identified a region of unknown polar behaviour, and labeled it as an anti-ferroelectric region (of unknown structure) in the phase diagram between ferroelectric low temperature phases and the paraelectric cubic phase for $x<15 \%$, which is a departure from the form of the phase diagram for PZT. Acceptable piezoelectric constant $\mathrm{d}_{33}$ values of $125 \times 10-12 \mathrm{CN}^{-1}$ near the MPB at room were found, although this is still a long way from the $\mathrm{d}_{33}$ values of PZT which typically lie between $300 \times 10-12 \mathrm{CN}^{-1}$ and $600 \times 10-12 \mathrm{CN}^{-1}$. Takenaka et al.'s phase diagram is reproduced from [16] in Fig. 2.1. Please note that this is an early view of the phase diagram which is considered wrong in many respects today. I show it to emphasise what was thought previously to my research on BNT-BT, and how my work has changed understanding of this material. 


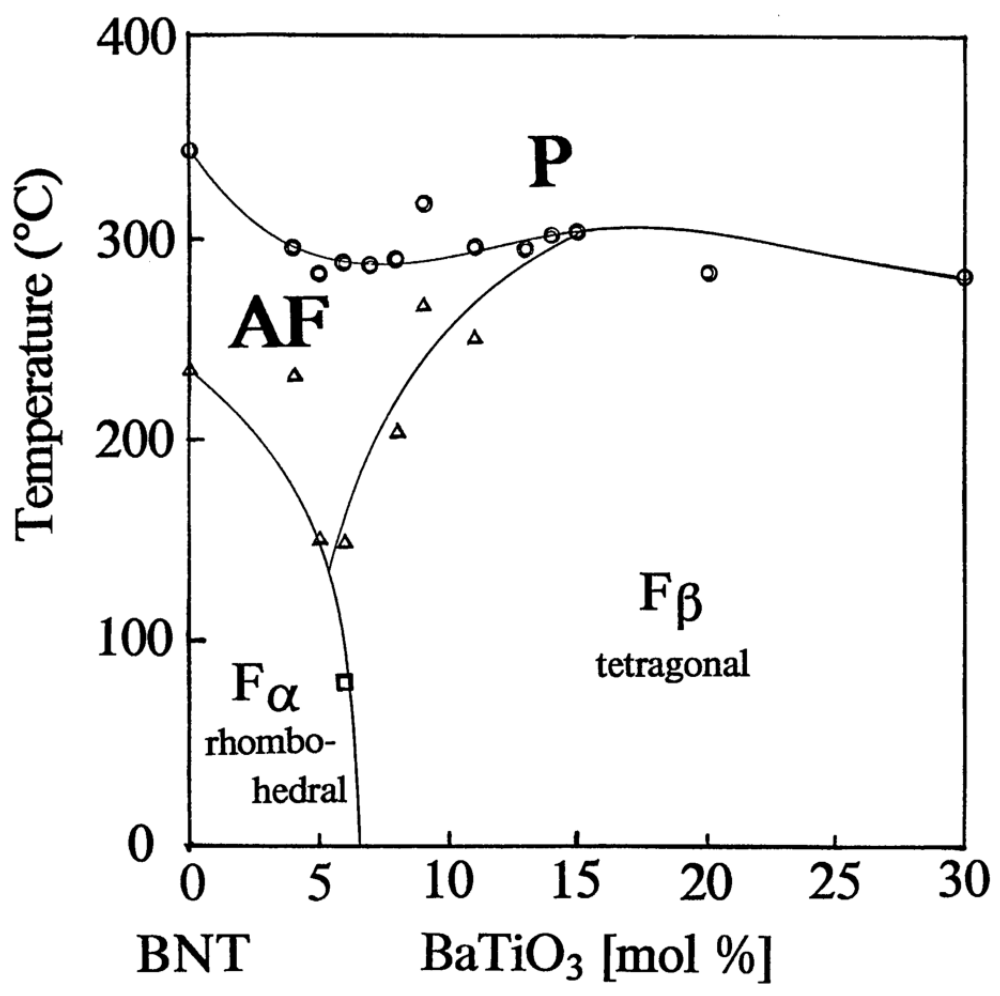

Figure 2.1: An early (1991) phase diagram of BNT-BT produced by bulk electronic measurements. Note the morphotropic phase boundary between the $\mathrm{F}_{\alpha}$ and $\mathrm{F}_{\beta}$ phases, and the triple point between those two phases and the AF phase (the top phase marked $\mathrm{P}$ is a paraelectric cubic phase). Although this phase diagram is now considered substantially incorrect, I include it to illustrate the state of knoledge of BNT-BT prior to my research. Copyright 1991 The Japan Society of Applied Physics, figure reproduced with permission from [16]. 


\subsection{Lead titanate nanowires}

Chapter 5 of this thesis concerns experiments performed on nanowires made of lead titanate (PT). Lead titanate was one of the first widely used and produced ferroelectric materials. It is an end member of the now far more common PZT solid solution. The material is well characterised, and well understood on macroscopic scales. There is, however, more scope for new research examining nanoscale structures of this material.

In nanowires, there exist many reasons to expect different behaviour from the bulk. There is a far greater ratio of surface atoms to bulk atoms in the nanowires compared to macroscopic ceramics giving greater instability to the material. The non-flat surfaces may also cause strain on the PT. There is a unique direction in the nanowires, and different symmetry than in the bulk material. The overall smaller scale will cause restrictions on the kind of patterns that can be adopted by mesoscopic structures such as domain walls and cystal grains. The result of these factors can be profound, and lead to new types of behaviour, including the emergence of new phases and phase transitions.

While it is true that this material is lead based, there are advantages to testing how a well understood system responds to these types of changes before diving into new materials. The knowledge gained will allow us to more effectively direct research in the future.

The particular nanowire samples studied in this work are synthesised through an unusual method, beginning with hydrothermal synthesis of nanowires in the 'PX' phase. The PX phase of lead titanate forms fibrous crystal bodies rather than a bulk solid crystal. TEM images of PX-phase wires have shown them to be single crystalline and rectangular prism-like in cross section. Previous work by the author and others had identified an unusual crystal structure in this phase as well[3]. First principles density functional theory computations were performed to match the structure to XRD spectra and to polarised Raman spectra of the hydrothermally synthesised wires (these calculations were not the contribution of the author of this thesis). Fig. 2.2 shows the structure. The crystal structure has a large unit cell with eight stoichiometric units in the cell. It consists of edge-shared $\mathrm{TiO}_{6}$ octahedra and edge-shared $\mathrm{PbO}_{5}$ pyramids; the pyramids and the octahedra 

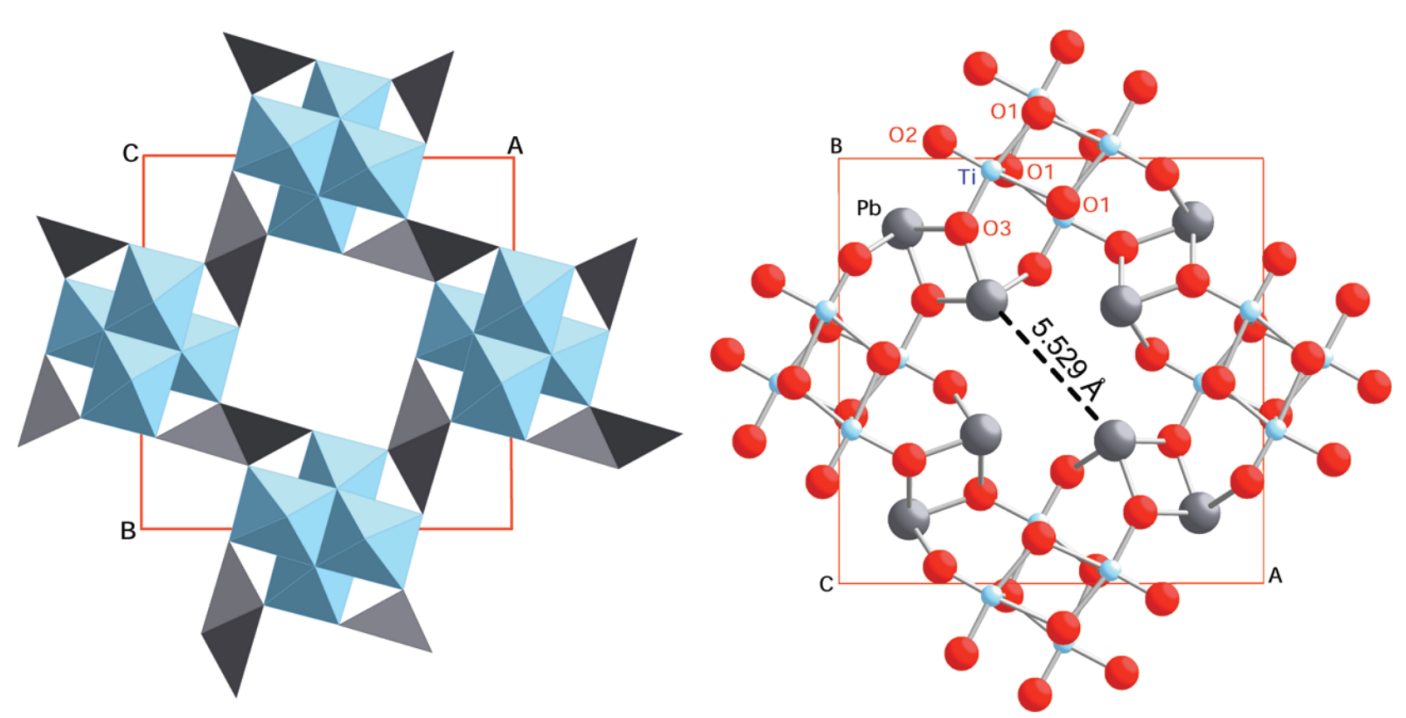

Figure 2.2: Structure of 'PX' phase of lead titanate, found in nanowires. Left: space filling geometrical model, and right: balls and sticks atomic model. Note the unusual gap in the structure, giving significantly reduced density in this phase. Reprinted (adapted) with permission from [3]. Copyright 2011 American Chemical Society.

are corner shared. It is a very different structure from the perovskite phase. Most interesting of all is the fact that there is a gap in this unit cell. An area in the middle of the cell has no atoms in it, a gap of minimum length 5.529 Åbetween two lead atoms. When the full crystal is built up by translation of the unit cells, these gaps line up and form cohesive channels through the material. One effect of these channels is that the density of this lead titanate phase is significantly less than the perovskite phase $\left(6.87 \mathrm{~g} / \mathrm{cm}^{3}\right.$ vs $\left.7.97 \mathrm{~g} / \mathrm{cm}^{3}\right)$. That difference in density causes some surprising results when the PX wires are transformed into the denser perovskite PT phase.

The wires can be transformed into normal perovskite phase lead titanate by annealing in air at $540{ }^{\circ} \mathrm{C}$, and when they are, they retain the nanowire morphology. Fig. 2.3 shows an SEM image of the annealed wires. This method of synthesis, contribution of Jin Wang, therefore is a new way of growing nanowires of lead titanate, which may have important technological applications. The structural perfection of these wires was unkown. It was expected that there may be large amounts of stacking defects in the nanowires due to the transformation into a more dense crystal structure. 


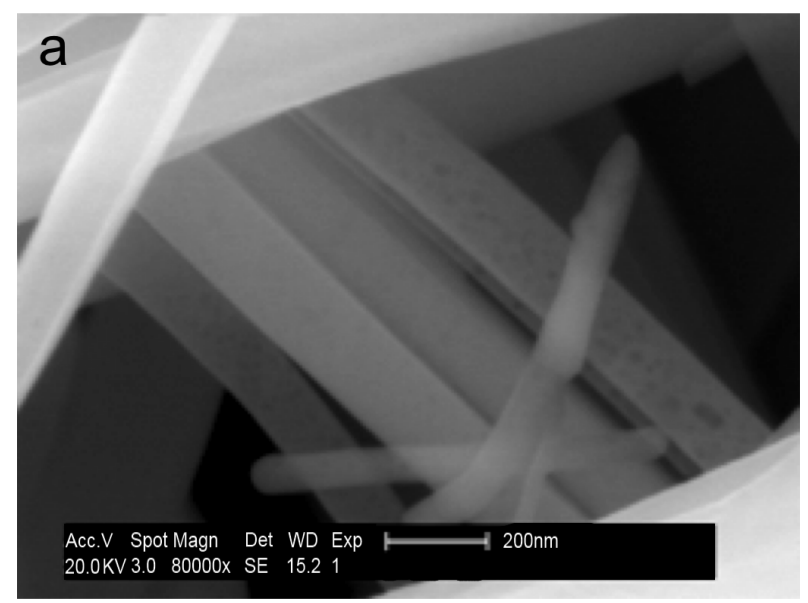

Figure 2.3: SEM image of PT nanowires produced via annealing of the less dense PX phase nanowires. The nanowire morphology can be seen to be retained through the annealing process.

Since Raman spectroscopy is an excellent tool for attaining structural information (by proxy) on small length scales, we decided to use this to try and detect signs of imperfections. One sign in particular that would be an easy to see indication of disorder in the wires is a lowering of the cubic-tetragonal phase transition temperature. So we performed temperature dependent Raman spectroscopy measurements on these wires to find out if that was the case.

Shown in Fig. 2.4 are Raman spectra of lead titanate single crystals at room temperature for reference, showing the spectrum of tetragonal PT. Cubic PT has no Raman active first order modes. The figure is reproduced from [17].

\subsection{STO Thin Films}

Strontium titanate is an interesting material case. It has been studied for many years due to its rich variety of phases displaying ferroic behaviour. These include anti-ferrodistortive[18], ferroelectric, anti-ferroelectric[19], and ferroelastic[20], each being expressed under the appropriate conditions. It is the ferroelectric properties that we are most interested in in the current study.

Like PT and BNT-BT, STO is a perovskite crystal, but unlike those mate- 


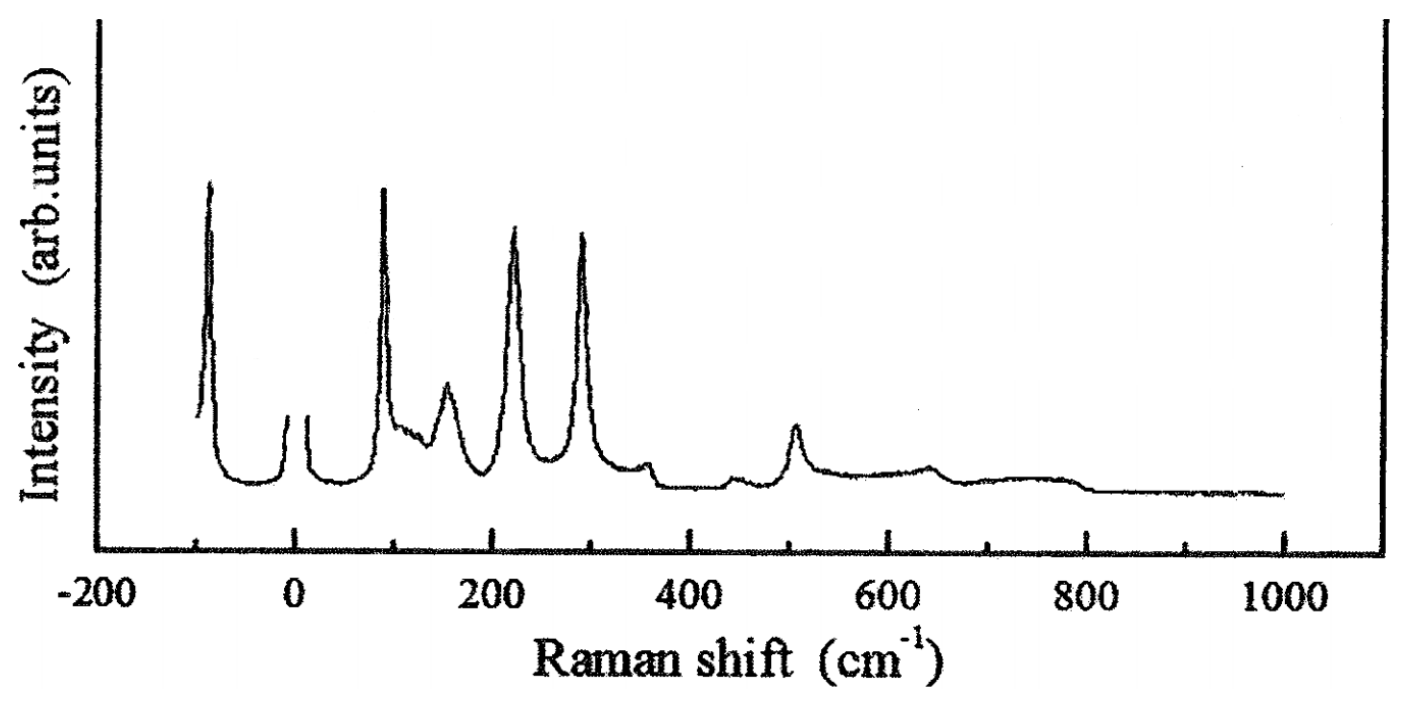

Figure 2.4: Raman spectrum of tetragonal (and ferroelectric) lead titanate for reference. The cubic (paraelectric) phase of lead titanate has no first-order peaks. (C)2007 IEEE, Figure reproduced with permission from [17].

rials, pure STO has no ferroelectric phase, even down to $0 \mathrm{~K}$. It does, however, display incipient ferroelectric behaviour (where the material is close to undergoing such a transition) at low temperatures. This can be seen in the behaviour of its dielectric susceptibility, and of its ferroelectric soft mode. The dielectric susceptibility of STO follows Curie-Weiss behaviour from high temperatures, apparently rising with a predicted Curie temperature of $38 \mathrm{~K}$. However, below about $70 \mathrm{~K}$, a deviation from Curie-Weiss behaviour is observed. The permittivity stops increasing, and instead levels out and becomes constant below 5-10 K [21]. The soft mode likewise starts softening as temperature is decreased, but the frequency stabilises and never completely softens [22]. This kind of behaviour has been called quantum paraelectricity, and is attributed to quantum fluctuations supressing the ferroelectric phase transition[23].

It does not take very much to overcome the quantum fluctuations and induce ferroelectricity in STO however. Electric fields, increased pressure, lattice strain, Ca substitution and ${ }^{18} \mathrm{O}$ isotopic substitution have all been shown to induce ferroelectricity in STO. STO is in fact very finely balanced, at a critical point between ferroelectricity and paraelectricity. Because of this, it makes a great playground in which to explore the mechanics of ferroeletricity and the influence that various factors have on it. 
Lattice strain is one such factor[24][25], and is is of great interest in technological applications. It is achieved by epitaxial growth on top of lattice-mismatched substrates, which can be relatively easily integrated into manufacturing processes. The 2D geometry also integrates well with existing electronic devices. Other advantages of this factor are the lack of other chemical modifications of the ferroelectric material required, and with it the need for fine control of dopant quantities, the ability of this factor to function well on the micro and nanoscales, and the passive nature of this factor, as compared to pressure or electric field induced ferroelectricity. One should be clear - there is no great interest in fabricating piezoor ferroelectric elements out of STO. Even with the best improvements, it still falls well short of the properties of PZT, or even of other less able piezoelectrics that see use. The interest here is to understand the effect of lattice strains upon ferroelectrics, with a view to using this factor to improve other materials which already display appealing properties.

The interest in lattice strain in STO followed on from observations of a ferroelectric phase transition occurring in STO under high pressure[26]. The effect of high pressure is to compress one or more of the crystal lattice constants, possibly preserving or possibly altering the symmetry of the unit cell. High pressure environments are impractical for most applications - they are tricky to maintain, and volatile. But lattice strain can be used to create an analogous compression of the crystal axes without the need for an externally pressurised environment. An additional advantage is that lattice strain can also create a corresponding expansion of crystal lattices, an option not available to pressure manipulation.

Chapter 6 of this thesis reports results of experiments performed on thin strained films of strontium titanate. The goal was to measure and understand the effect of lattice strain on STO through any changes visible in the Raman spectrum, particularly focused on the ferroelectric phase transition. In order to understand and make inferences from the spectra we recorded, there is a wealth of literature that must be presented. The important background for interpreting the Raman measurements is given in this section.

Pure single crystals of STO are cubic at room temperature[27]. At $105 \mathrm{~K}$, they undergo an anti-ferrodistortive (AFD) phase transition which is characterised by rotation of the oxygen octahedra about the c-axis in the material[28]. Adjacent 

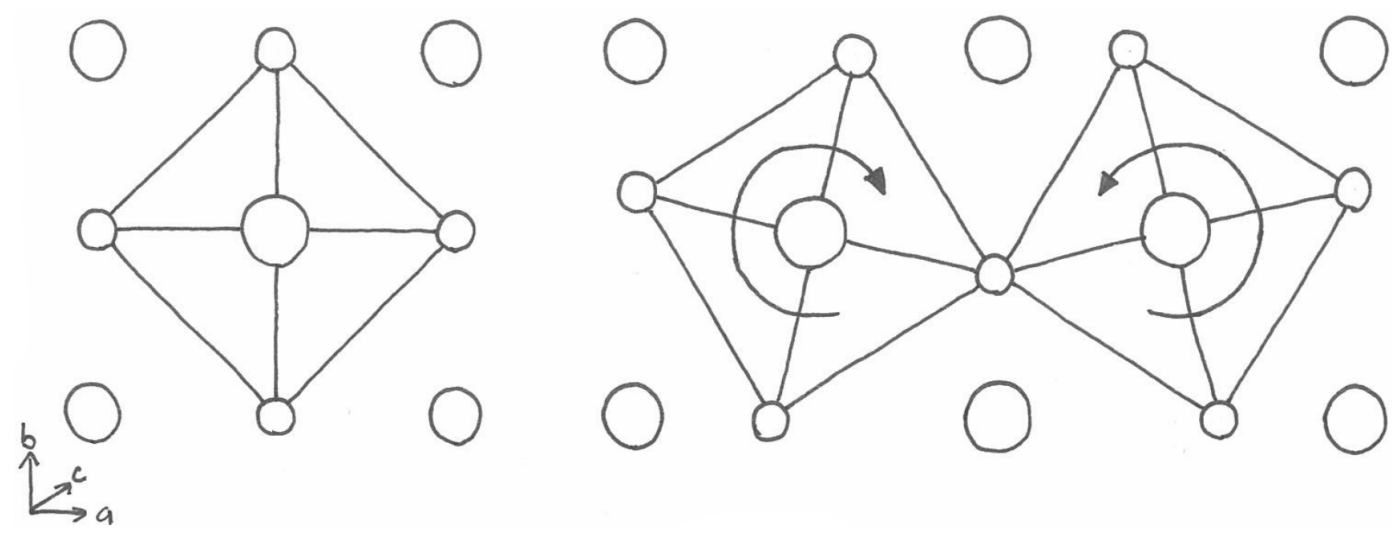

Figure 2.5: Left: crystal structure in the cubic phase of STO, z-projection. Right: crystal structure of the AFD phase of STO characterised by anti-phase rotation of adjacent oxygen octahedra about the z-axis. Note the doubling of the unit cell caused by this transition.

octahedra rotate in opposite directions, resulting in an expansion of the unit cell, and reduction of symmetry. This distortion is illustrated in Fig. 2.6. The material after this transition is no longer isotropic, but it is still paraelectric. And as mentioned in a previous section, the material shows signs of a frustrated ferroelectric phase transition at temperature below $35 \mathrm{~K}$, and a ferroelectric phase of this material is accessible by various perturbations. The ferroelectric phase usually has an orthorhombic structure, and Raman spectra have been taken of it in this phase so we have examples for comparison. With no alterations to the perfect bulk crystal however, STO undergoes no further phase transitions.

Some of the earliest attempts at creating thin films of STO were unsuccessful, yielding films with greatly reduced permittivity, and it was discovered in later years that this was due to bad film growth quality - small grain sizes with large grain boundaries, cracking and delaminating faults, dislocation defects etc. The poor crystal quality had a huge effect on the electrical properties, suppressing ferroelectricity and dielectric permittivity[29] and hardening the ferroelectric soft mode[30]. As this was recognised, and better film growth methods were applied, results started to improve[31]. Modern interest in ferroelectric STO, and in strainengineering of ferroelectrics was sparked by a paper in Letters to Nature in 2004[1]. The authors, Haini et al., had grown thin films of STO via reactive molecular beam epitaxy on $\mathrm{DyScO}_{3}$ (DSO) [110] and $\left(\mathrm{LaAlO}_{3}\right)_{0.29} \times\left(\mathrm{SrAl}_{0.5} \mathrm{Ta}_{0.5} \mathrm{O}_{3}\right)_{0.71}$ (LSAT) [100] substrates. These substrates had lattice mismatch of $+1 \%$ and $-0.9 \%$ re- 
spectively. The films were $50 \mathrm{~nm}$ thick, low enough to limit the amount of strain relaxation. They then performed dielectric measurements on the material, and found a very strong and clear peak in the dielectric permittivity of the STO/DSO sample, peaking at $293 \mathrm{~K}$ with a value of nearly $\epsilon_{r}=7000$. This was a strong indication of a ferroelectric phase transition, raised from below zero to almost $300 \mathrm{~K}$ - a hugely significant increase in phase transition temperature. They did not find evidence of ferroelectricity in the STO/LSAT film. Further confirmation of ferroelectricity in these films in the form of polarisation hysteresis loops was provided later by [32]. Much work has been done since then, both theoretical and experimental on strained thin films of STO, and other materials in order to understand and reproduce this enormous enhancement of Curie temperature. But, due to experimental difficulties, little work has been reported on Raman measurements of strained STO thin films. This work presents temperature dependent Raman spectra on STO strained on three different substrates, relating them to other types of measurements and to theory.

\subsubsection{Enhancing Raman signals from thin films using UV excitation}

While methods of thin film growth and fabrication are well established, methods for characterising thin films are more difficult to achieve. It is difficult to detect the signal from such small layers as $<100 \mathrm{~nm}$, and traditional methods are being replaced. Raman spectroscopy is no exception. Measuring the spectra of strained thin films presents us with difficulty experimentally: the scattered intensity measured from such films is greatly reduced compared to bulk, this is due to the much smaller scattering volume that the laser is able to address. The scattering volume for a typical laser Raman spectroscopy experiment has a cross sectional area on the order of a $1 \mu \mathrm{m}$ diameter circle, and its extent into the sample is dependent upon the optical absorption of the material. Silicon, as an example material has an attenuation length of several micron in the visible part of the electromagnetic spectrum. In the case of a $100 \mathrm{~nm}$ thin film however, the penetration is limited to $100 \mathrm{~nm}$, resulting in a scattering volume that is smaller by an order of magnitude or more. Films used in this study are between $30 \mathrm{~nm}$ and $100 \mathrm{~nm}$ thick.

Though annoyingly long and time consuming, the former problem could be 
solved by increasing integration times for each measurement. Other factors such as stability of the sample, temperature controller and laser power have to be considered in such measurements, and any experiment requiring repeated measurements at slightly different parameters (such as temperature dependence) becomes agonizingly time consuming. However, for strained thin films we are faced with another compounding problem - interference from the substrate.

When the laser light has penetrated through the thin film, it doesn't just go away. It travels further into the substrate, where it can and will undergo Raman scattering processes, and get collected by our spectrometer and obfuscate the signal from the thin film. For most applications, one can solve this problem simply by using a material which is Raman inactive. However for the measurement of strained thin films, one is severely limited in what substrate materials one can use. One needs to select a substrate with the exact right lattice spacing and geometry, while keeping within an acceptable range of mechanical and chemical inertness parameters. The thermal expansion of the substrate must be considered as well. It is therefore rare that one has the luxury of choosing a Raman inactive material, and in fact with each substrate used in this study, there is a prominent Raman signal from the substrate material that must be dealt with.

For these reasons, it is highly desirable to investigate the possibility of enhancing the amplitude of the Raman signal from thin films, as well as the contrast over the substrate. The method we chose used ultraviolet (UV) excitation to reduce the penetration depth for greater surface selectivity. UV Raman spectroscopy is done the same way as normal Raman spectroscopy, except that the laser used for excitation is in the UV region of the electromagnetic spectrum. The technique, and its application to ferroelectric thin films was brought to attention by an article in Science magazine in 2006[33].

UV excitation has three main advantages over visible excitation for collecting Raman spectra from thin ferroelectric films. First of all, our materials of interest: the ferroelectric perovskites, while largely transparent in the visible, are strongly absorbing in the UV. Penetration depth of optical fields into the material are greatly reduced as a consequence. For example, in the ferroelectric material $\mathrm{BaTiO}_{3}$, penetration depth $(1 / \alpha)$ at $514.5 \mathrm{~nm}$ (green) excitation is on the order of several micron, whereas at $325 \mathrm{~nm}$ (ultraviolet) excitation the penetration depth 
is reduced to about $50 \mathrm{~nm}$. This absorption drastically reduces the scattering from the substrate, this scattering being one of the main obstacles to measuring signals from thin films. The second main factor in the advantage of UV is that ferroelectric materials tend to have band gaps in the region of $300-350 \mathrm{~nm}$. The presence of allowed electron transitions near the energy of excitation (and, as Raman scattering alters only slightly the light wavelength, near the energy of the scattered light too) results in a resonant enhancement of Raman scattering. Lastly, all Raman scattering increases with increasing excitation frequency, there is an $\omega^{4}$ factor in the equation for scattering cross section[34]. The first of these three factors is specific to the materials being studied (though it would apply to other wide band-gap materials also), the second applies specifically to thin film systems, and the third factor is of general application to the measurement of weak Raman signals.

However, UV has disadvantages as well, mostly related to greater losses in optical elements such as lenses and mirrors at this frequency compared to visible light. In particular, it is difficult to craft lenses with high numerical aperture in the UV. Specialised mirrors and mirror coatings can be crafted with reflectance nearly as good as visible light mirrors, but it is much harder to find suitable materials with high refractive indices in this region of the spectrum. The advantages of UV measurements are offset by the poorer optical transmission through excitation of the sample, and collection of the Raman scattered light. These experimental drawbacks make UV Raman worse overall for most applications, hence the more common use of visible or near infrared excitation light.

The experimental apparatus for making the UV Raman measurements were designed and created for use in the measurements of STO thin films, details are provided in section 3.2.3. The method was successful, allowing us to reveal new information about lattice dynamics in our STO samples.

\subsubsection{Raman features of STO}

Although only a limited number of Raman spectra of strained STO thin films have been published so far, it is important for the interpretation of our Raman spectra that we have a solid understanding of the Raman features of STO under all kinds of perturbations. What follows is a list of Raman features of STO available in the 
literature.

Second order features

In the cubic phase of STO (highest temperature phase), only second order features are present in the Raman spectrum[27]. First order features are Raman silent due to symmetry considerations. There are four notable bands visible. At room temperature, these bands are:

- $80 \mathrm{~cm}^{-1}$ narrow peak

- $250-450 \mathrm{~cm}^{-1}$ broad band. Multiple peaks

- $600-750 \mathrm{~cm}^{-1}$ broad asymmetric band. Multiple peaks

- $1040 \mathrm{~cm}^{-1}$ weak broad peak

As temperature decreases, the $80 \mathrm{~cm}^{-1}$ band decreases in intensity, becoming undetectable below $40 \mathrm{~K}$. the $250-450 \mathrm{~cm}^{-1}$ band also undergoes dramatic changes upon lowering temperature, becoming asymmetric, losing much of its high-frequency intensity. The $600-750 \mathrm{~cm}^{-1}$ lowers in intensity but is otherwise unaltered with dropping temperature, and the $1040 \mathrm{~cm}^{-1}$ band does not change at all. Fig. 2.6 is reproduced from [27] and shows the second order features in STO, as they vary with temperature.

First order hard modes associated with AFD distortion

Once STO has undergone its AFD distortion, the lowering of symmetry activates several first order Raman modes. The doubling of the unit cell caused by this distorion folds vibrational modes from the R-point of the cubic Brillouin zone to the zone centre in the AFD Brillouin zone. These modes therefore become Raman active, as the momentum conservation condition is now satisfied in the new symmetry. 


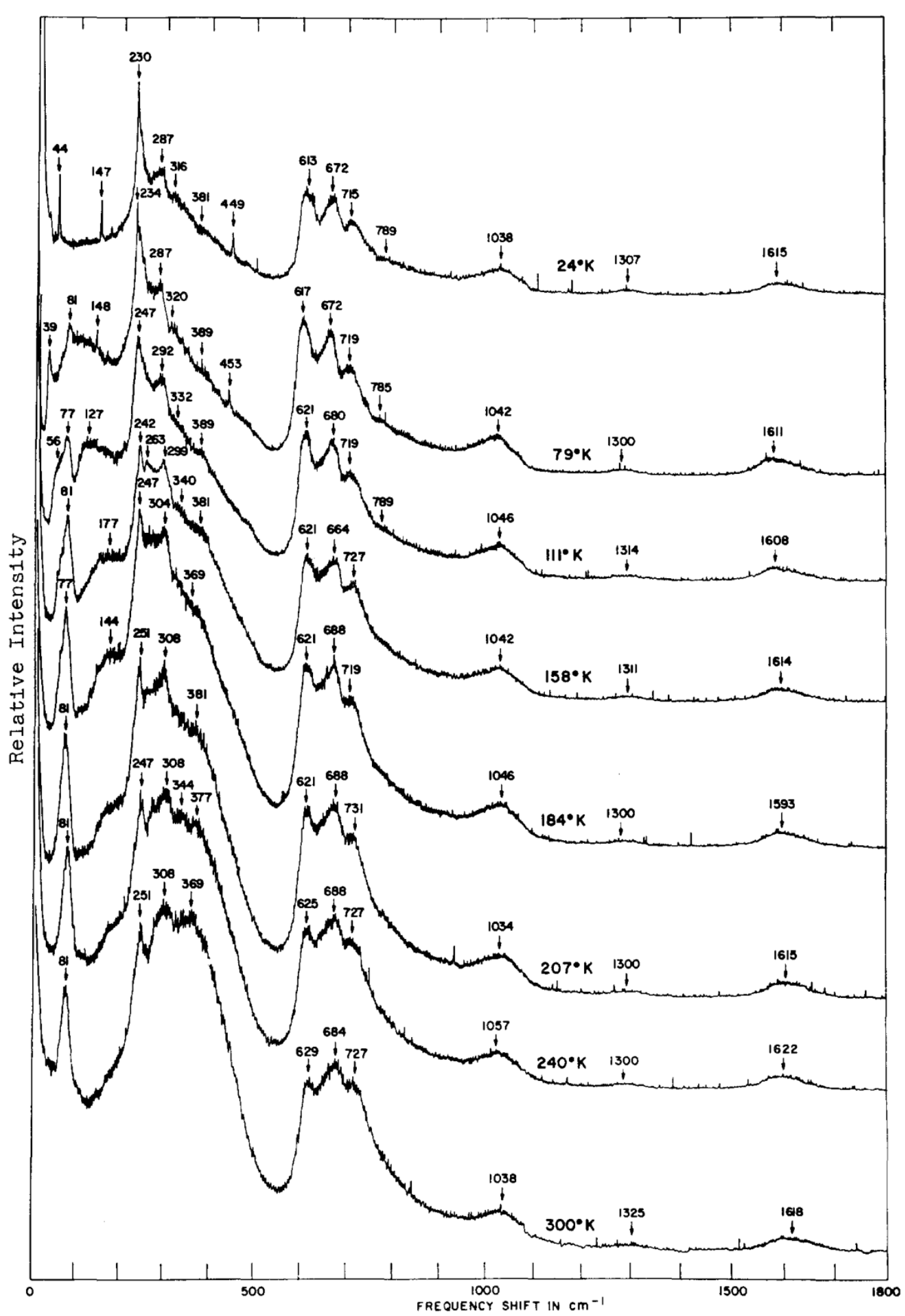

Figure 2.6: Temperature dependent Raman spectra of STO. The spectra have been vertically offset for ease of viewing. The second-order features and R-point modes activated by the AFD transition can be seen in these spectra. Reprinted with permission from [27]. Copyright 1968, American Institute of Physics. 
- $145 \mathrm{~cm}^{-1}$, symmetry $\mathrm{E}_{g}$ in tetragonal phase

- $230 \mathrm{~cm}^{-1}$, symmetry $\mathrm{B}_{2 g}$ in tetragonal phase

- $450 \mathrm{~cm}^{-1}$, symmetry $\mathrm{B}_{1 g}$ in tetragonal phase

These modes appear suddenly in the spectrum after the STO undergoes an AFD phase transition and the remain reasonably constant in amplitude and in frequency down to $0 \mathrm{~K}$. These modes can be seen in the spectra of Nilsen and Skinner, Fig. 2.6, with further spectra and analysis of the second order and AFD modes in [35]

Anti-ferrodistortive soft mode

The AFD soft mode is Raman inactive in the cubic phase. Below the AFD transition, the mode is activated, and it has two components of different symmetry[19]. The frequencies stated below are the near- $0 \mathrm{~K}$ frequencies of the modes.

- $15 \mathrm{~cm}^{-1}$, symmetry $\mathrm{E}_{g}$ in tetragonal phase

- $50 \mathrm{~cm}^{-1}$, symmetry $\mathrm{A}_{1 g}$ in tetragonal phase

These soft modes both soften to zero frequency as the anti-ferrodistortive phase transition temperature is approached. Petzelt et al. notes that the frequency of the $\mathrm{A}_{1 g}$ symmetry soft mode is a measure of the octahedral tilting (the AFD primary order parameter), and that the splitting between these two soft modes is a measure of the secondary order parameter: the tetragonal distortion[36]. Of special note is that the $\mathrm{E}_{g}$ symmetry soft mode is further altered if the material undergoes a ferroelectric phase transition. This $\mathrm{E}_{g}$ mode is split into two components of different frequency as the symmetry is lowered from tetragonal to orthorhombic, one at $15 \mathrm{~cm}^{-1}$ and one at $20 \mathrm{~cm}^{-1}$. Figs. 2.7 through 2.9 are reproduced from [19], [37], and [38] respectively. They show the AFD soft modes in various samples of STO, as described in their captions. 


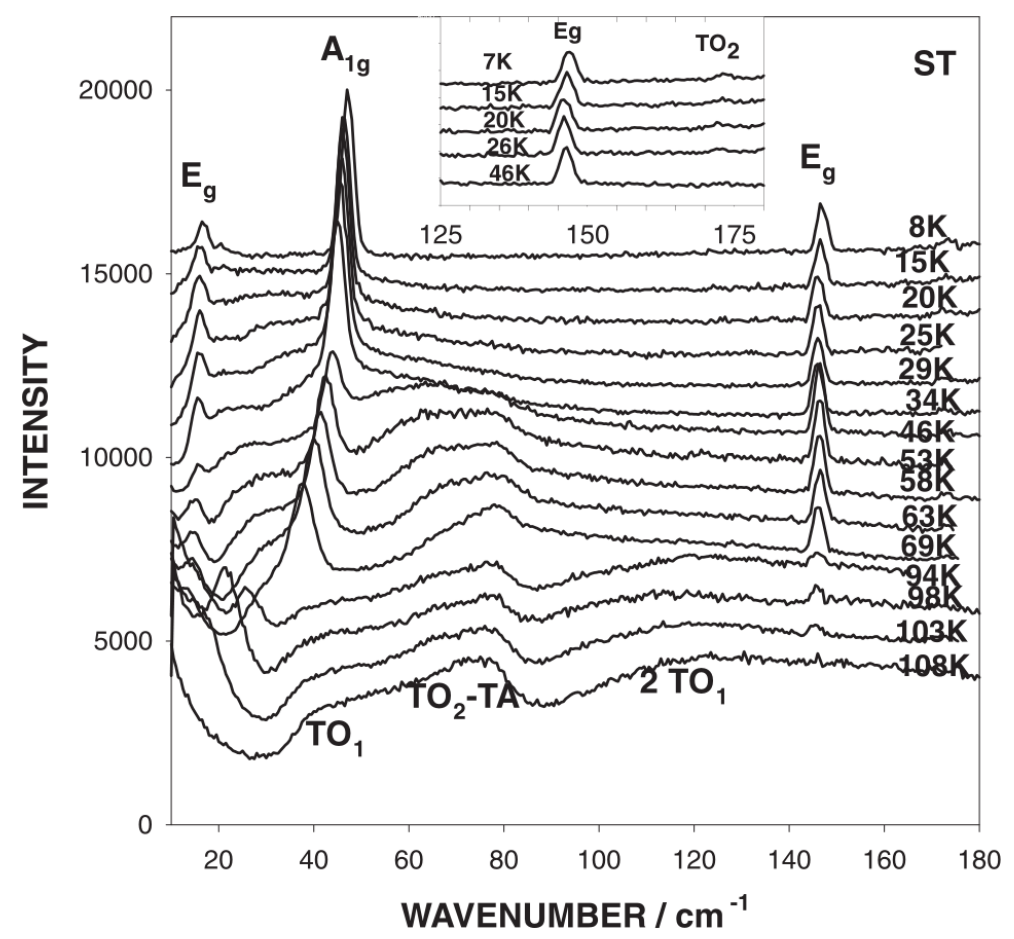

Figure 2.7: Raman spectra of STO showing the $\mathrm{E}_{g}$ and $\mathrm{A}_{1 g}$ components of the AFD soft mode (and the hard mode at $150 \mathrm{~cm}^{-1}$ ). The spectra have been vertically offset for ease of viewing. Figure reproduced with permission from [19]. 


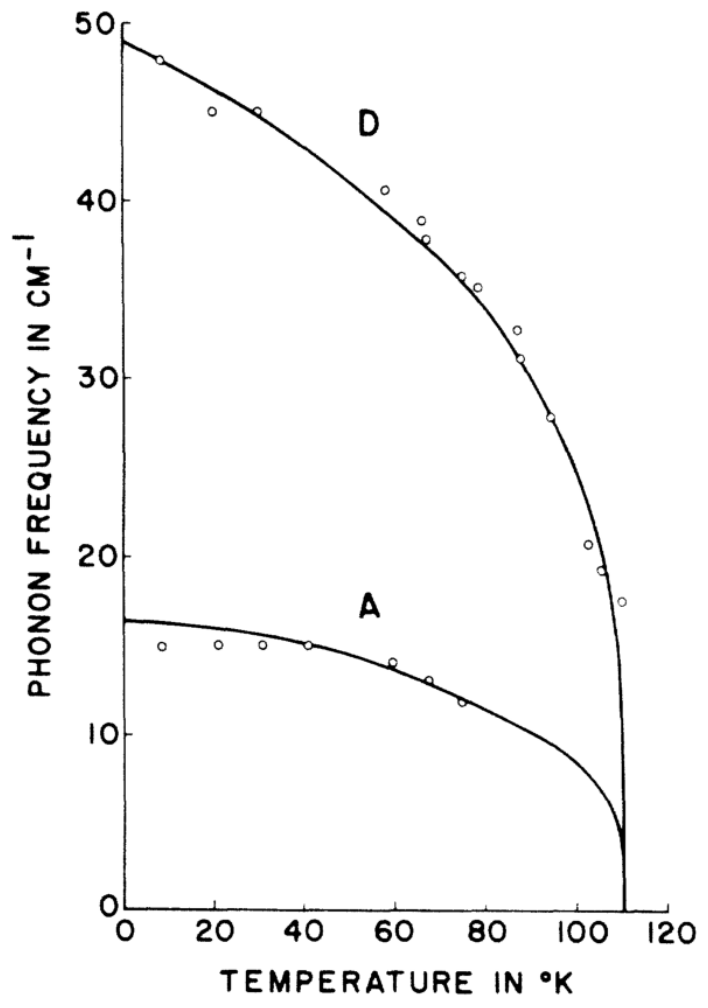

Figure 2.8: Temperature dependence of the AFD soft mode frequency. Reprinted figure with permission from[37]. Copyright 1968 by the American Physical Society. 


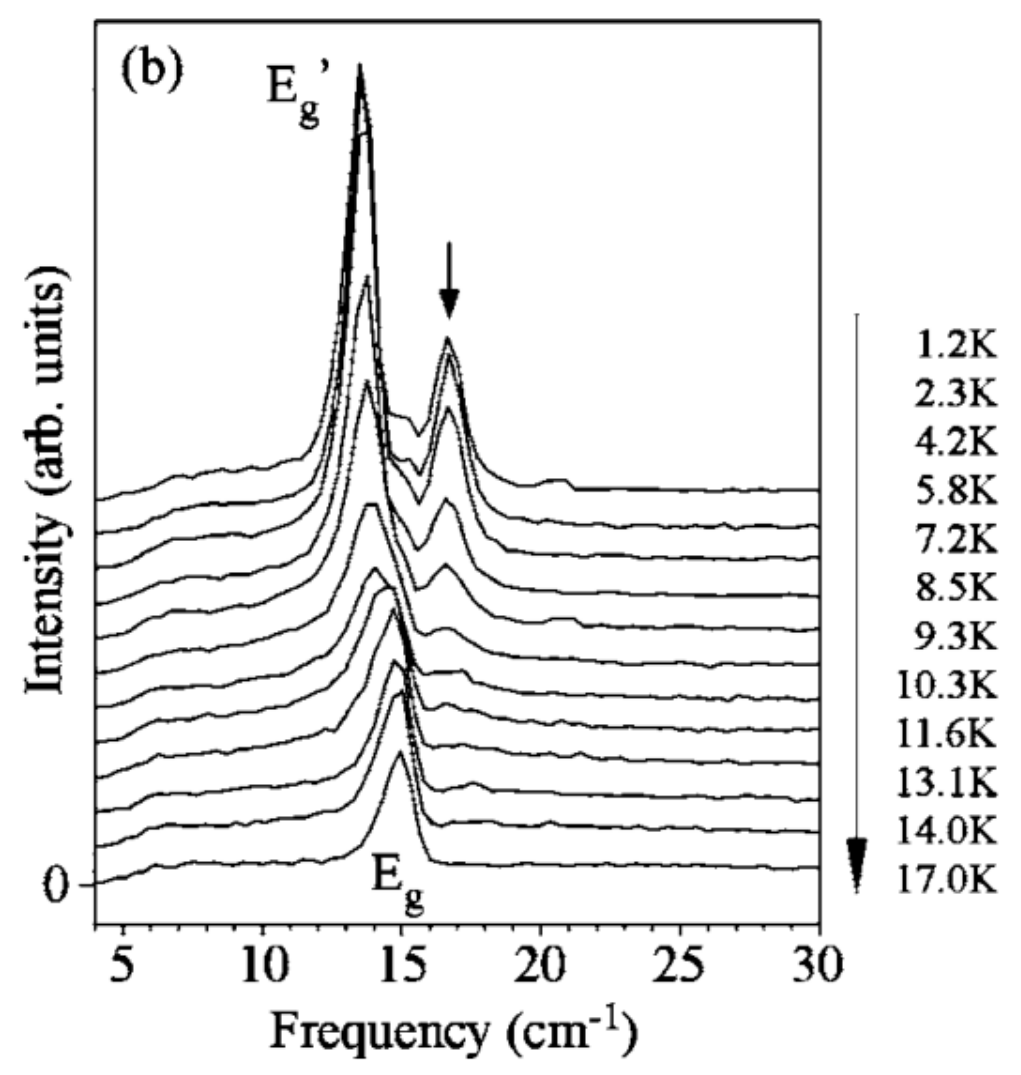

Figure 2.9: Raman spectra showing the splitting of the $\mathrm{E}_{g}$ component of the ferroelectric soft mode upon phase transition to the orthorhombic ferroelectric phase, shown in this case in ${ }^{18} \mathrm{O}$ substituted STO. The spectra have been vertically offset for ease of viewing. Reprinted figure with permission from[38]. Copyright 2005 by the American Physical Society. 
First order hard modes associated with FE distortion

These modes are first order and are Raman forbidden in the cubic phase. However, they are often seen in Raman spectra of cubic phase STO nonetheless, made active by defects in the lattice, grain boundaries, disorder or strain. Typically these factors create polar micro-regions (PMR) in which these phonon mode transitions are Raman active[39]. This explains their presence in the Raman spectra at low intensities. The frequencies of these modes, and their assigned names in the literature are listed below[40], and an example of Raman spectra showing these modes is found in Fig. 2.10.

- $175 \mathrm{~cm}^{-1}\left(\mathrm{TO}_{2}\right)$

- $265 \mathrm{~cm}^{-1}\left(\mathrm{TO}_{3}\right)$

- $500 \mathrm{~cm}^{-1}\left(\mathrm{LO}_{3}\right)$

- $550 \mathrm{~cm}^{-1}\left(\mathrm{TO}_{4}\right)$

- $800 \mathrm{~cm}^{-1}\left(\mathrm{LO}_{4}\right)$

Different samples reported in literature display different combinations of these peaks. $\mathrm{TO}_{2}$ and $\mathrm{TO}_{4}$ are the most consistently seen across all samples. In cases of (near) displacive phase transitions such as those in calcium substituted STO or ${ }^{18} \mathrm{O}$ isotope substituted STO, the appearance of these first order modes is sudden, and indicative of a first order phase transition. However, in most other samples in which these peaks are seen, such as pure ceramics, thin films, non-stoichiometric samples etc., these peaks increase in intensity only gradually with decreasing temperature over a course of more than $100^{\circ} \mathrm{C}$. In some samples, small intensities of these modes persist up to room temperature, or even higher. In the latter, weak temperaturedependence case they are typically cited as a sign of polar micro-regions in the material.

The $\mathrm{TO}_{2}$ hard mode is often seen to have a strong asymmetry which can be modeled with a Fano function[41]. This is given as evidence of the polar microregions, showing, it is argued, the existence of a continuum of states interacting 
with the polar hard mode[40]. The theory behind this is that the Fano asymmetry arises from the interaction between a mode of a well defined frequency with a broad continuum of states. In the case of STO disordered polar microregions are usually assigned as the reason for the broad continuum.

The $\mathrm{TO}_{3}$ mode is one of the more rarely observed hard modes. In several papers, it is argued that this mode only appears in the presence of a distortion that has a spatial extent on the order of a phonon wavelength or longer, and that the observation of this mode then implies a long-range lowering of symmetry in the material[2]. This argument is on the basis of observations in a different perovskite, potassium lithium tantalate (KLT). The argument goes: The $\mathrm{TO}_{3}$ mode in perovskite structured materials is non-polar, while the other TO and LO modes are polar. Therefore, $\mathrm{TO}_{3}$ cannot be activated by polar micro-regions in the same way that the other hard modes can be. There must be a true structural distortion which breaks centrosymmetry to activate this mode. The anti-ferrodistortive phase, while tetragonal, is still centrosymmetric, so it will not show the $\mathrm{TO}_{3}$ mode. In the case studies below, all of the samples for which the STO does not undergo a ferroelectric phase transition do not display this peak. Where it has been seen in STO, the peak has quite a low intensity compared to the polar TO modes, and even to second order scattering. It is sharp enough to be picked out individually, however.

Ferroelectric soft mode

The ferroelectric soft mode in STO is called $\mathrm{TO}_{1}$ in the tetragonal AFD phase. This name comes from IR measurements, in which the mode is active at all temperatures. It has $\mathrm{E}_{u}$ symmetry in the AFD phase, and splits into three components below the ferroelectric phase transition. The low temperature frequencies as measured in Bianchi et al. are given below[43].

- $5 \mathrm{~cm}^{-1}$

- $10 \mathrm{~cm}^{-1}$

- $15 \mathrm{~cm}^{-1}$ 


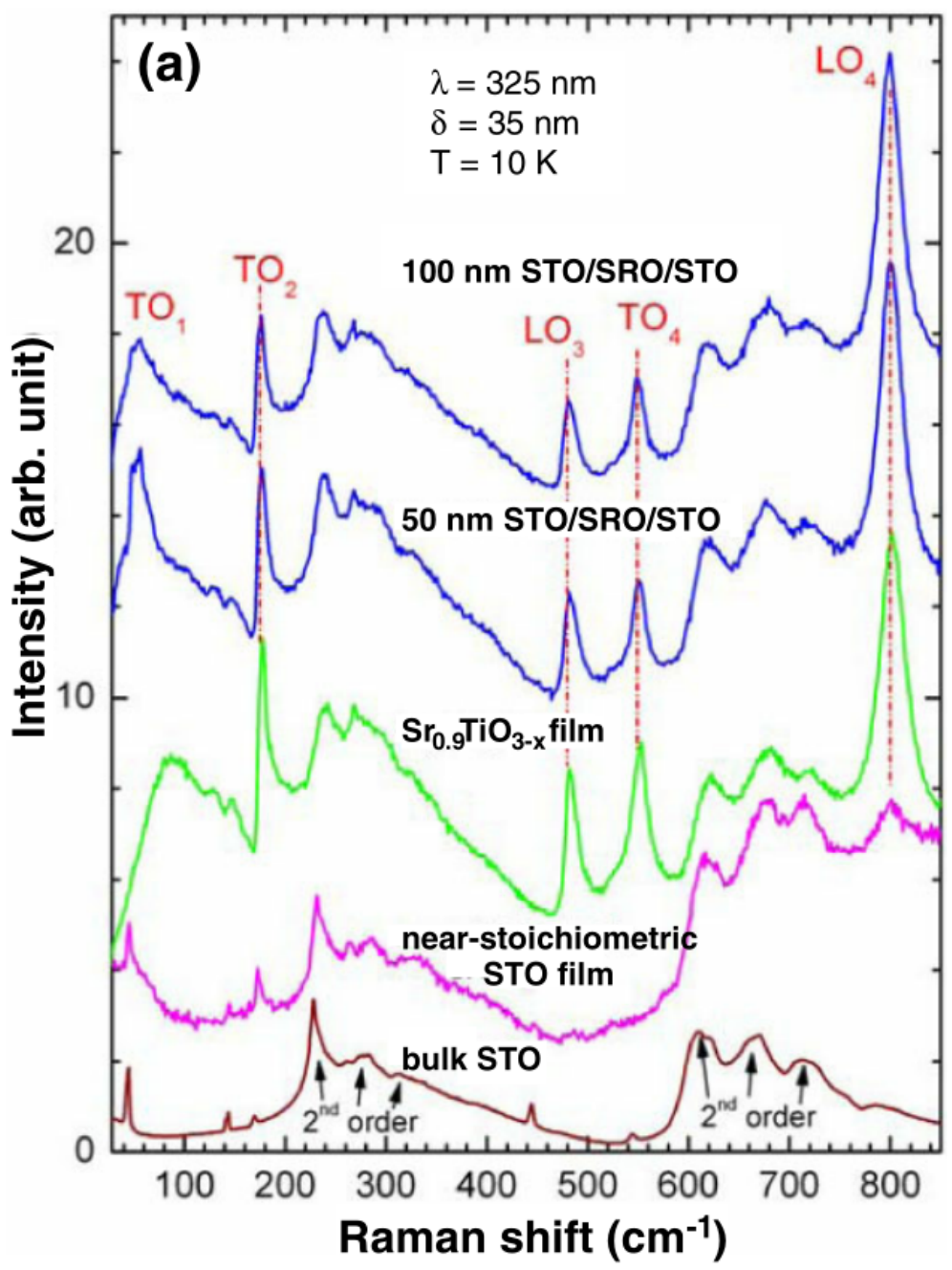

Figure 2.10: Raman spectra of STO showing first-order scattering modes (labeled in red). The spectra have been vertically offset for ease of viewing. Reprinted figure with permission from[42]. Copyright 2010 by the American Physical Society. 


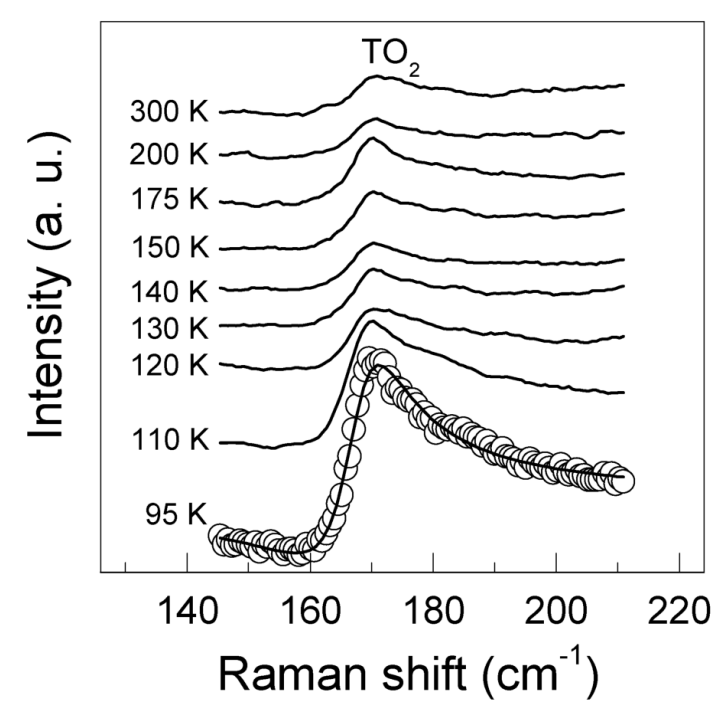

Figure 2.11: Close up of the $\mathrm{TO}_{2}$ mode in polycrystalline thin films. The spectra have been vertically offset for ease of viewing. Note the strongly asymmetric shape of the mode in this spectrum. Figure reproduced with permission from [40].

The low temperature splitting into three components shows the ferroelectric phase to have greatly lowered symmetry. In $0.7 \%$ Ca substituted STO this transition occurs at around $18 \mathrm{~K}$. This mode is tremendously intense in the ferroelectric phase, compared to all other modes present. Its proximity to the laser line makes it difficult to detect however. The ferroelectric soft mode in the ferroelectric phase of Ca-substituted STO is shown in Fig. 2.13, reproduced with permission from [43]. Above the phase transition, this phonon is Raman disallowed. However, there is in STO a weak second order Raman peak which follows the temperature/frequency behaviour of twice the $\mathrm{TO}_{1}$ mode as measured by infrared spectroscopy, where the $\mathrm{TO}_{1}$ mode is active. This second order Raman peak can be confidently assigned as $2 \mathrm{TO}_{1}$.

Also present in many STO samples is PMR-activated $\mathrm{TO}_{1}$ scattering mentioned in the previous section. This peak follows closely the softening of the $\mathrm{TO}_{1}$ mode in pure STO, and has the small intensity expected of limited volume defect induced scattering. Interestingly, signs of this scattering can be seen even in the nominally pure single crystals studied by Nilsen and Skinner. Fig. 2.14 is reproduced with permission from [27]. It shows the weak PMR activated soft mode scattering in a nominally pure STO single crystal. 


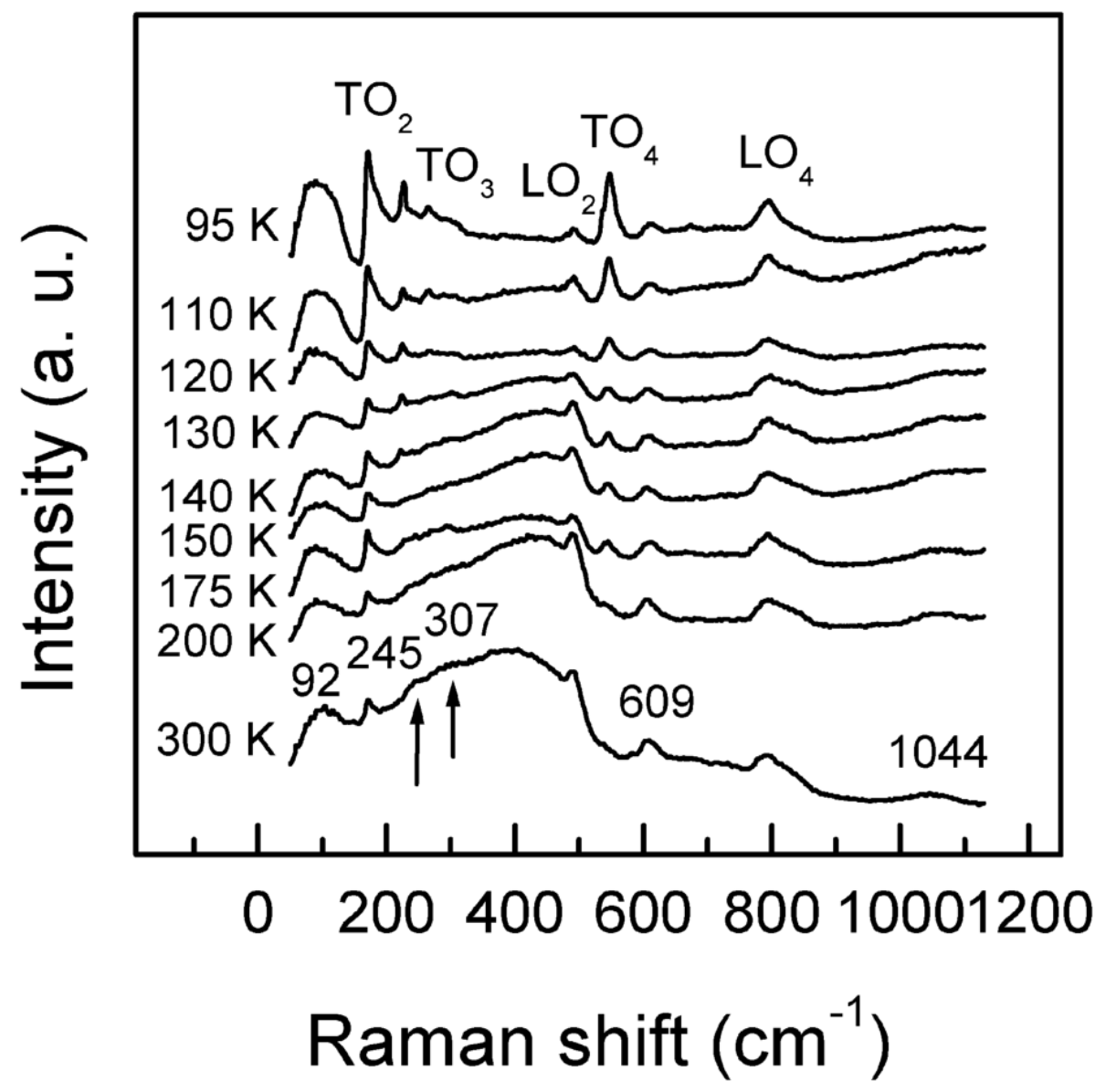

Figure 2.12: Temperature dependent Raman spectra of polycrystalline STO thin films. The spectra have been vertically offset for ease of viewing. In this Raman spectrum, the $\mathrm{TO}_{3}$ mode can be seen. Figure reproduced with permission from [40]. 

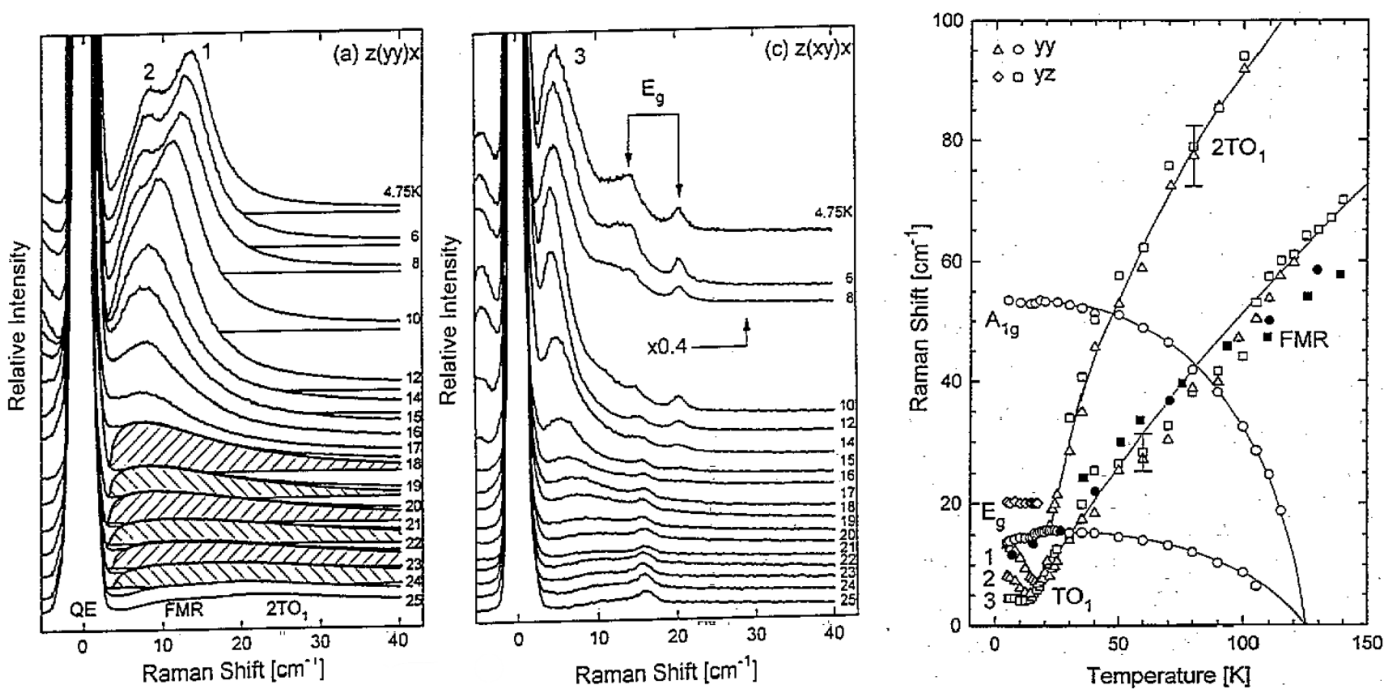

Figure 2.13: Left: Raw spectra and Right: frequency-temperature dependence of ferroelectric soft mode in Ca-substituted STO. The spectra have been vertically offset for ease of viewing. In this material, there is a ferroelectric phase below $20 \mathrm{~K}$. The softening to near zero and the splitting into three components below $\mathrm{T}_{C}$ can be seen in these figures (splitting of the $\mathrm{E}_{g}$ component of the AFD soft mode can also been seen in this figure). In the right hand panel of this figure, FMR refers to Raman scattering activated by ferroelectric microregions. Figure reproduced with permission from [43].

There is another feature in the Raman spectrum of STO which some authors associate with FE distortions. It has a frequency of between 70 and $120 \mathrm{~cm}^{-1}$, and is a relatively strong, broad, single peak (this is a separate peak from the $80 \mathrm{~cm}^{-1}$ second order feature). This mode is likely also the ferroelectric soft mode, judging by infrared measurements of the $\mathrm{TO}_{1}$ mode in various systems[44][45]. Some influences such as grain boundaries, cracks and other imperfections can cause the mode to display far less softening than in the pure STO case, resulting in a mode of greatly elevated frequency. It is often activated in the Raman simultaneously with other first order STO modes in polar microregions, although the mode at this elevated frequency is not a sign of ferroelectricity.

\subsubsection{Specific examples}

Now that we know the language of the STO Raman spectrum, we will examine several examples of STO systems with various syntheses and alterations. These 


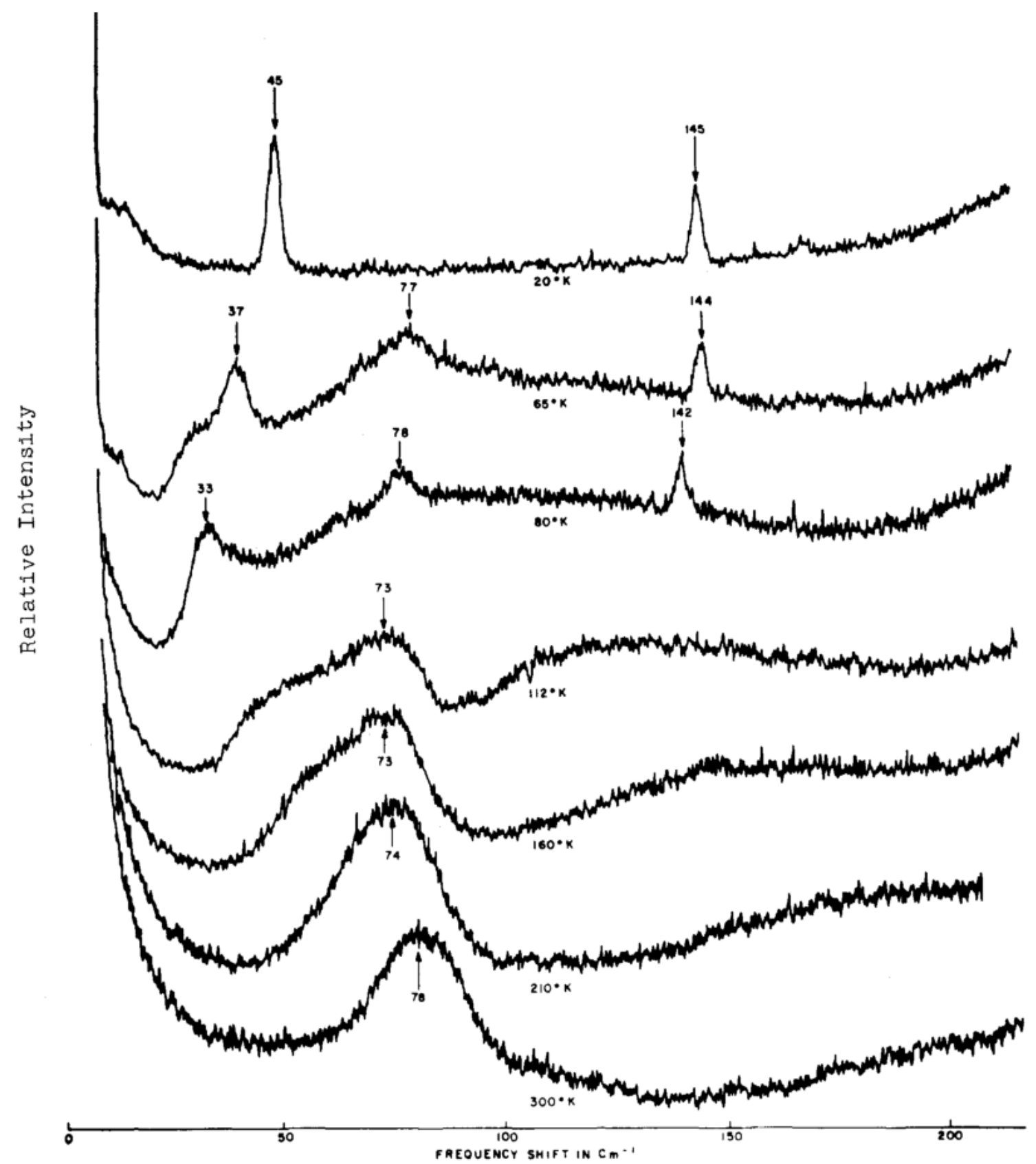

Figure 2.14: Close up of the low-frequency range of Raman spectra on pure single crystal STO. The spectra have been vertically offset for ease of viewing. The shoulder that develops on the low-frequency side of the $74 \mathrm{~cm}^{-1}$ peak and subsequently softens is scattering from the ferroelectric soft mode. This mode softening is incomplete down to $0 \mathrm{~K}$ in pure STO. Reprinted with permission from [27]. Copyright 1968, American Institute of Physics. 
case studies will take us more into detail on the behaviour of the STO modes under various conditions, and where they can be linked with ferroelectric phases or not. The pure STO single crystal case (work of Nilsen and Skinner[27]) was used to describe the second order features, and will not be discussed further here except to point out that the same group fabricated a second single crystal that was discoloured by impurities, and that in this crystal small peaks were detected at 176 and $554 \mathrm{~cm}^{-1}$, the $\mathrm{TO}_{2}$ and $\mathrm{TO}_{4}$ peaks. This shows that only a small amount of impurities are necessary to activate these modes. All of the experiments reported in these case studies are the work of other groups, and not the author.

Case study 1: Ca-substituted single crystal STO

This case study examines the work of Bianchi et al.[43] and Ouillon et al.[19]. The former studies a single crystal of STO with Ca-substitution in the a-site cation. $0.7 \%$ of $\mathrm{Sr}$ has been replaced with Ca. In this crystal, the usual second order spectrum is observed at room temperature. The AFD hard and soft modes start to be detected at $125 \mathrm{~K}$, a slightly elevated temperature from the bulk case.

Scattering regions associated with $\mathrm{TO}_{1}$ scattering and $2 \mathrm{TO}_{1}$ are observed to high temperatures. The $\mathrm{TO}_{1}$ scattering is symmetry disallowed, although as we saw even in single crystals it can be weakly seen. The appearance of the $\mathrm{TO}_{1}$ scattering in the crystal is explained by local symmetry breaking regions - tiny volumes that nucleate around defects, primarily $\mathrm{Ca}$ ions. The defects polarise the small regions around them, breaking the symmetry, and thus allowing first order Raman scattering. This phenomena is also sometimes referred to as ferroelectric microregions, and it has been well studied in STO and other highly polarisable materials[46]. Higher dielectric permittivity and lower temperatures cause the regions to grow. As the material is able to distort from smaller E-fields, distortions penetrate deeper into the material, polarising larger volumes. Lowering of the defect concentration causes this scattering to disappear, and this is observed in ultra pure $\mathrm{KTaO}_{3}$.

Fig. 2.13 (reproduced from [43]) shows important features of the Raman spectrum of this Ca-substituted STO sample. At $20 \mathrm{~K}$ the $\mathrm{TO}_{1} \mathrm{FE}$ soft mode has soft- 
ened to approximately $5 \mathrm{~cm}^{-1}$, and at this point it splits into three components, whose frequency begin rising again and whose amplitude dramatically increases with falling temperature. It is necessary to measure the material in multiple different polarisation conditions in order to see all of the components. Simultaneously, in this sample the splitting of the $\mathrm{E}_{g}$ symmetry AFD soft mode into two components was observed. The splitting of these modes prompted the authors to assign orthorhombic symmetry to the ferroelectric mode below $20 \mathrm{~K}$ in this sample. Finally, simultaneous with the splitting of these modes, the appearance of $\mathrm{TO}_{2}$ and $\mathrm{TO}_{4} \mathrm{FE}$ hard modes is observed. Fig. 2.15 is reproduced from [43] and shows the temperature dependence of these modes in open circles. They show a shape similar to a Fermi-Dirac distribution, with a saturation at low temperature, a drop off of intensity around the phase transition temperature, here at $15 \mathrm{~K}$, and a tail of intensity above the transition temperature. The width of the entire shape, from high saturation to low saturation is in this case approximately $20 \mathrm{~K}$. This pattern cannot be verified is not exactly a Fermi-Dirac distribution. Accordingly, in the rest of this thesis I will simply refer to this pattern as an 'S-shaped curve'.

This material has been independently verified to be ferroelectric with a $\mathrm{T}_{C}$ dependent on Ca level by dielectric measurements and poling[47]. The polarisation directions were quoted as the [110] pseudocubic directions of these crystals in this study, and it was noted that this contrasts to the polarisation directions of STO where ferroelectricity had been induced by uniaxial stress, which were [100].

It can be concluded that small amounts of calcium substitution in STO leads to the emergence of a low temperature ferroelectric phase, $\mathrm{T}_{C} \leq 35 \mathrm{~K}$. The transition is into a orthorhombically structured material with ferroelectric polarisation directions along the [110] pseudocubic directions. The main indicators of this phase transition in the Raman spectrum are the splitting of the AFD $\mathrm{E}_{g}$ soft mode and the $\mathrm{FE} \mathrm{E}_{u}$ soft mode into two and three components respectively, the critical softening of the FE soft mode, and the appearance, and rapid rise in intensity of polar hard modes $\mathrm{TO}_{2}$ and $\mathrm{TO}_{4}$.

Studies have also been done on STO crystals with higher levels of Ca-substitution. Fig. 2.16 is reproduced from [19], and shows the polar mode intensity temperature dependence for such samples. Very low frequency data was not available in this study, but there are some interesting differences in the temperature dependence of 


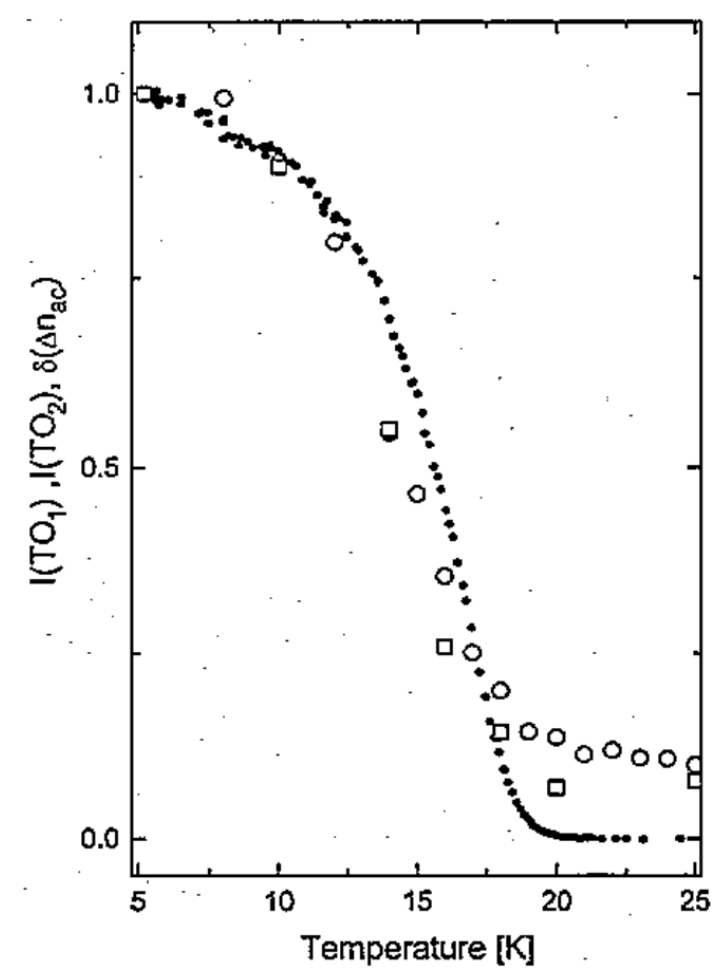

Figure 2.15: Temperature dependence of the first-order mode intensity in Casubstituted STO. Open squares: $\mathrm{TO}_{1}$ mode, open circles: $\mathrm{TO}_{2}$ mode, closed circles: dielectric loss in this material for comparison. Note the rapid rise to saturation in a curve somewhat like a Fermi-Dirac distribution. In the remainder of this thesis, this kind of temperature dependence will be referred to as an S-shaped curve. Figure reproduced with permission from [43]. 

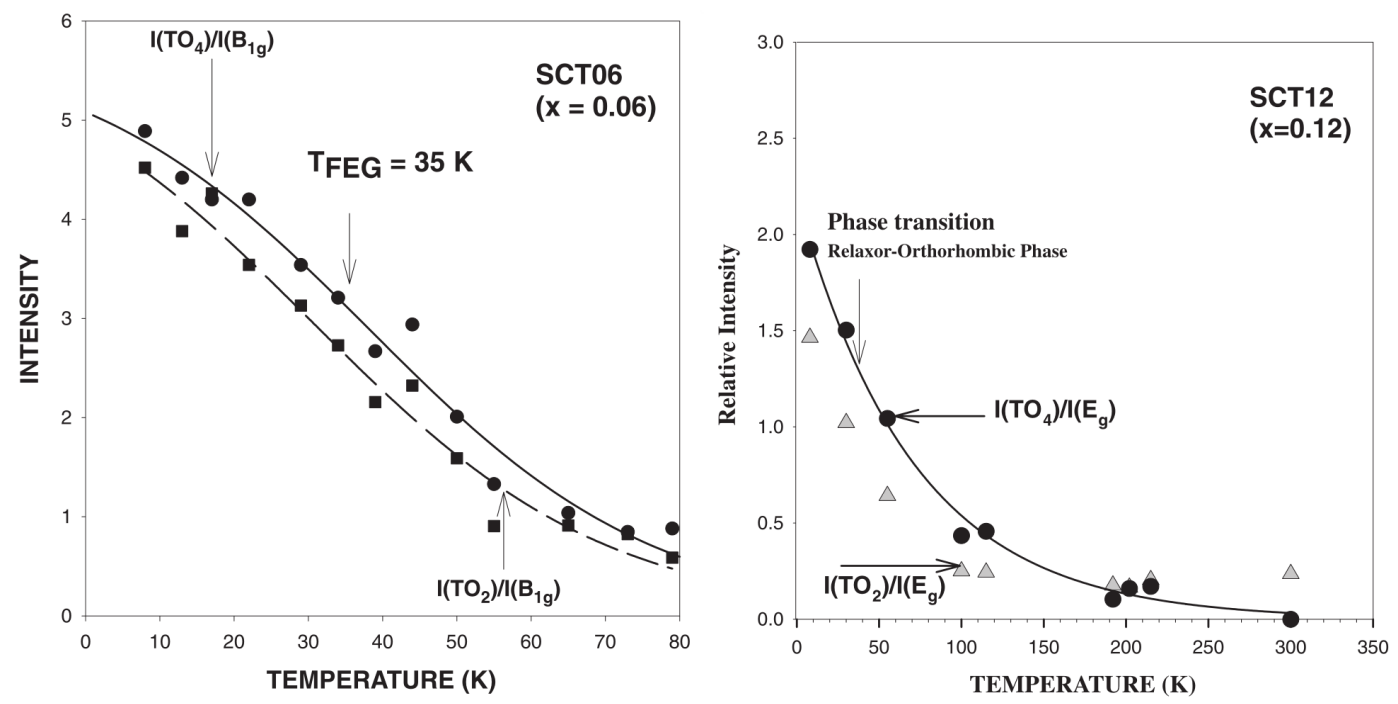

Figure 2.16: First-order mode intensity vs temperature in STO with higher Casubstitution levels. Both $\mathrm{TO}_{2}$ and $\mathrm{TO}_{4}$ modes are displayed, as marked Left: $6 \%$ Ca, Right: $12 \%$ Ca. Note the same S-shaped curve being shown as in 2.15, but spread out much broader in temperature. Figure reproduced with permission from [19].

the $\mathrm{TO}_{2}$ and $\mathrm{TO}_{4}$ modes. The S-shaped curve noted in the $0.7 \%$ Ca substituted case persists, but higher Ca substitution results in the broadening of this S-shaped curve. In the $6 \%$ substituted case, the S-shaped curve has a width of about $80 \mathrm{~K}$, and in the $12 \%$ substituted case it has a width of over $200 \mathrm{~K}$. Because the effect on the central position of the s-curve is less than the broadening, in the latter case the material never reaches a saturation point in terms of its polar hard mode intensities. In addition to the changes in the temperature dependence of the polar hard modes, a splitting in the $150 \mathrm{~cm}^{-1} \mathrm{Eg}$ mode is seen at low temperatures in both samples. The authors of this study assign it to an additional orthorhombic distortion related to local polar ordering, distinct from the AFD tilt. This transition occurs at around $80 \mathrm{~K}$ for $6 \% \mathrm{Ca}$, and around $100 \mathrm{~K}$ for $12 \% \mathrm{Ca}$. Finally, in the $12 \%$ Ca sample only, two additional modes appear below $30 \mathrm{~K}$ at $80 \mathrm{~cm}^{-1}$ and $125 \mathrm{~cm}^{-1}$. These modes are similar in position and behaviour to modes found in anti-ferroelectric 30\% Ca SCTO, and are assigned as such. Conversely, studies have also been done on smaller levels of Ca-substitution in STO[48]. These did not show complete softening of the ferroelectric soft mode, nor splitting in any of the modes. 
Fig. 2.17 is reproduced from [47], and shows the permittivity for samples of various Ca substitution levels. In the dielectric permittivity, the peak height reduces, and the width broadens with increasing Ca substitution from $0.7 \%$ to $12 \%$. Nevertheless, there does still exist a peak at $12 \%$, so one concludes that the $6 \%$ and $12 \%$ samples are both still ferroelectric, albeit weakly, and that their Raman spectra therefore show features that can be associated with ferroelectric STO. The data on the temperature dependence of $\mathrm{TO}_{2}$ and $\mathrm{TO}_{4}$ in this material shows us that a saturation of polar mode intensity is not a strict necessity for a ferroelectric phase of STO. It does, however, show a clear link between the magnitude of the Curie permittivity peak of STO and the temperature range over which the polar mode intensities are changing in the Raman spectrum. The broader a temperature range, the weaker the permittivity peak.

Case study 2: ${ }^{18}$ O substituted STO

The experiments described in this case study are done by Taniguchi et al. [38]. These samples are single-crystal STO pieces which have been baked in an atmosphere of ${ }^{18} \mathrm{O}$ gas, resulting in the uptake of ${ }^{18} \mathrm{O}$ atoms into the STO replacing ${ }^{16} \mathrm{O}$. Raman spectra from two samples are given: one with $23 \%{ }^{18} \mathrm{O}$ substitution, and one with $32 \%{ }^{18} \mathrm{O}$ substitution. The group sought to induce a ferroelectric phase in STO above room temperature by replacing some of the oxygen ions with heavier isotopes. This would be expected to change the lattice dynamics, and the hope was that given the fine balance in STO it would be sufficient to change the phase diagram. They were successful in creating ferroelectric STO samples by this method.

Raman data is shown in the frequency range of $4-80 \mathrm{~cm}^{-1}$ in Fig. 2.18, reproduced from the [38]. In this range, both AFD soft modes are seen. Below $10 \mathrm{~K}$, the $\mathrm{E}_{g}$ AFD soft mode splits into two components for the $32 \%$ substituted case, while the $23 \%$ substituted case does not show this splitting. Concurrently to the splitting, the $32 \%$ substituted sample shows the emergence of the $\mathrm{E}_{u} \mathrm{TO}_{1}$ ferroelectric soft mode. The soft mode is shown not to soften all the way to zero, and at temperature $10 \mathrm{~K}$, the mode begins hardening again, reaching a frequency of $8 \mathrm{~cm}^{-1}$ at $1.2 \mathrm{~K}$, which was the lowest temperature measured. This fact that the 


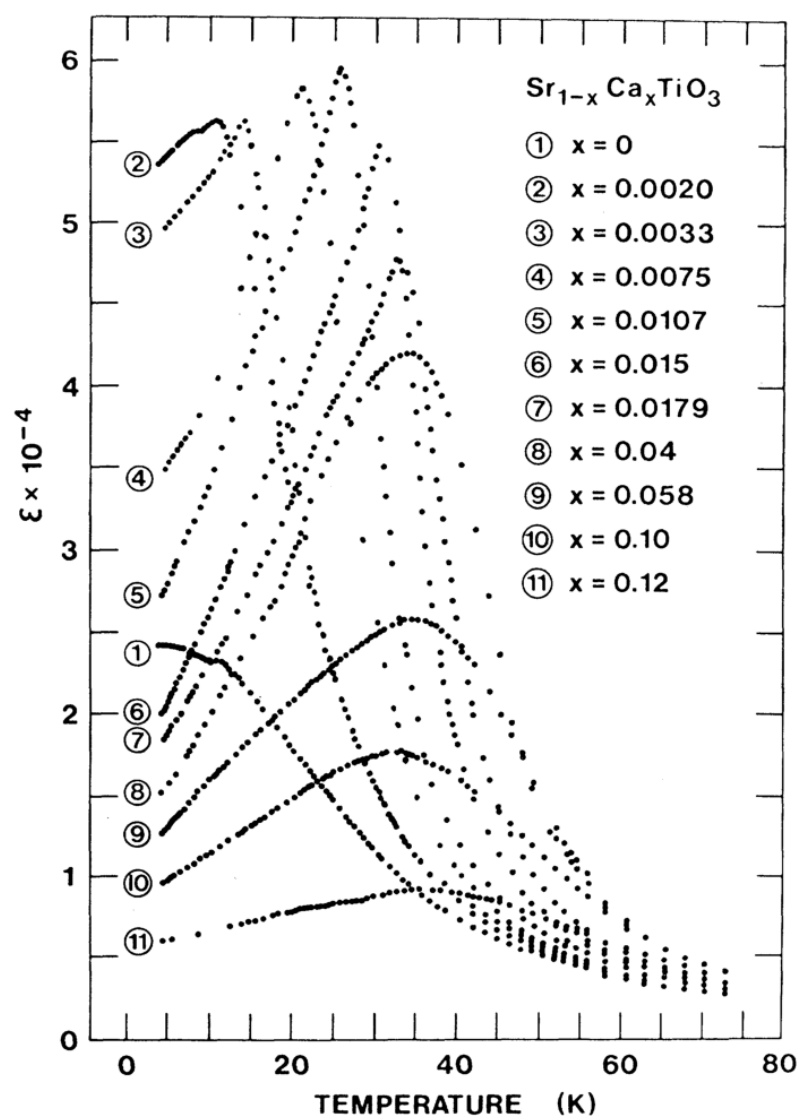

Figure 2.17: Dielectric permittivity of STO with various levels of Ca-substitution, as marked. $0.2 \%$ substitution is sufficient to induce a ferroelectric phase. The phase transition temperature and peak permittivity both increase up to $1.07 \%$. Subsequently, the peak permittivity falls and broadens, and the transition temperature stagnates at around $37 \mathrm{~K}$. Reprinted figure with permission from[47]. Copyright 1984 by the American Physical Society. 
mode does not soften to zero is interpreted by the authors as being caused by the simultaneous contributions to the Raman spectrum of ferroelectric regions, and paraelectric regions in the crystal. In this study the ferroelectric soft mode $\left(\mathrm{TO}_{1}\right)$ appears to have only a single component below the nominal transition temperature. However, as this peak was only shown by the authors in one polarisation, this result is not necessarily inconsistent with the three component splitting displayed by the calcium-substituted samples of Bianchi et al. Some of the polarisations shown by Bianchi et al. show only a single peak of this mode. It is worth noting that the incomplete softening discussed by the authors of this paper is also seen in the Bianchi paper.

Electrical measurements have been carried out on ${ }^{18} \mathrm{O}$ substituted STO samples [21][49]. The measurements have shown them to be ferroelectric, by peaks in the electrical permittivity, and by D vs E hysteresis loops when the substitution factor is high enough. $25 \%$ substitution is low enough that it still results in the quantum paraelectric behaviour of pure STO, but above this concentration ferroelectric anomalies in the permittivity are seen. In addition, the peak permittivity values are higher even than in pure STO. This contrasts to the Ca-substituted samples, for which the permittiviy peaks were slightly below the max value of permittivity in pure STO.

The $32 \%{ }^{18} \mathrm{O}$ substituted single crystal sample is therefore another example of STO that has become orthorhombic and ferroelectric, signaled in the Raman by the splitting of the $\mathrm{E}_{g} \mathrm{AFD}$ mode, and the emergence of the $\mathrm{E}_{u} \mathrm{TO}_{1}$ soft mode to intensities significantly higher than the second-order STO features.

Case study 3: Pure ceramic STO

The next system to examine is pure ceramic STO, experiments reported by Petzelt et al.[36]. In this system, as in the previous one, the AFD phase transition is found to be slightly elevated, in this case to $130 \mathrm{~K}$. It is interesting to note however that the $\mathrm{E}_{g}$ component of the AFD soft mode in the low temperature phase softens to an unusually high frequency $-40 \mathrm{~cm}^{-1}$ compared to $18 \mathrm{~cm}^{-1}$ in the pure single crystal case. The authors suggest that the high frequency of this 

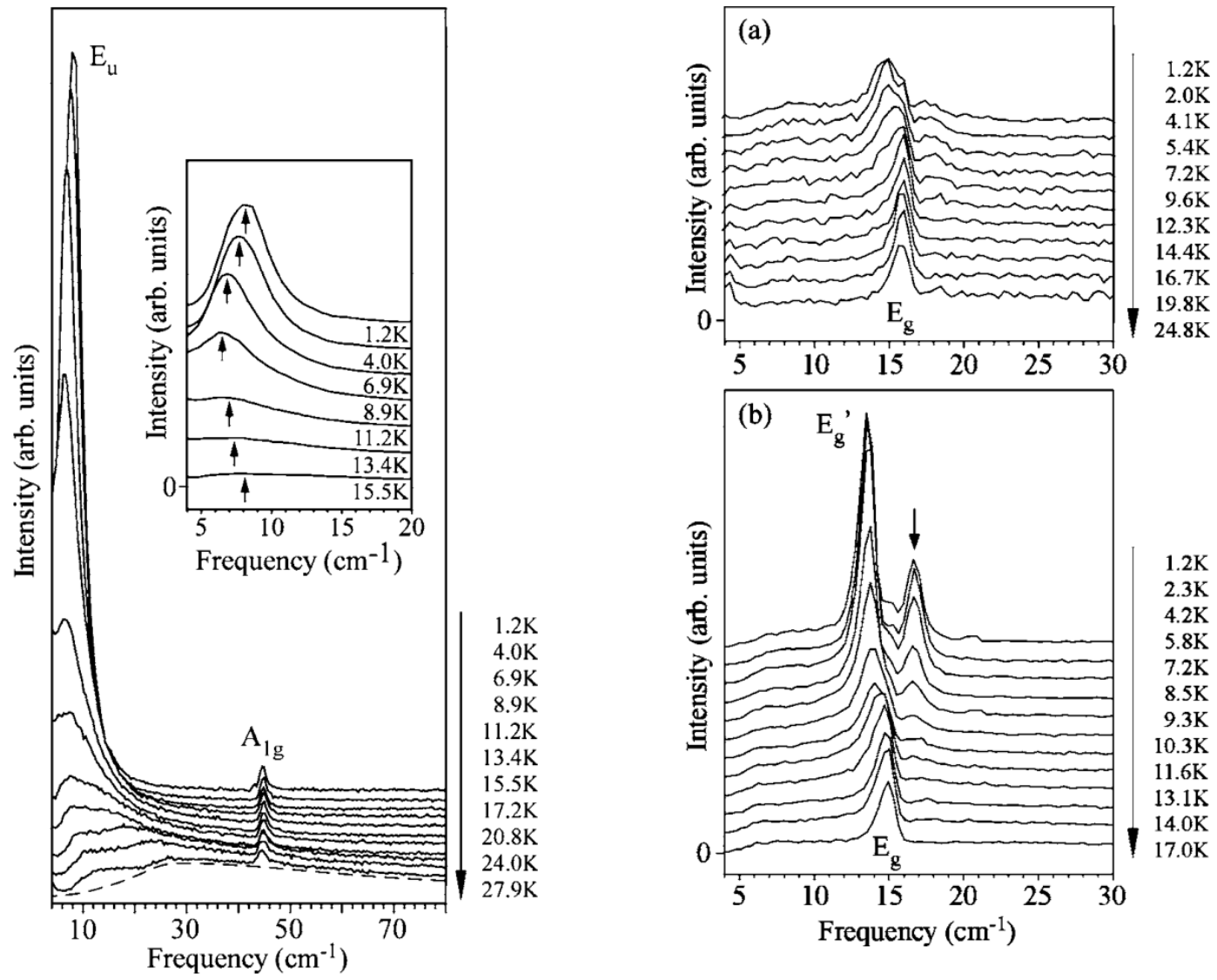

Figure 2.18: Raman spectra of ${ }^{18} \mathrm{O}$ substituted STO. The spectra have been vertically offset for ease of viewing. Left: The activation of the ferroelectric soft mode can be seen. These spectra collected in $x(y y)-x$ geometry. Right, a) $23 \%{ }^{18} \mathrm{O}$ substituted STO and b) $32 \%{ }^{18} \mathrm{O}$ substituted STO. These spectra collected in $x(y z)-x$ The splitting of the AFD soft mode can be seen in b) compared to the spectra resulting from no phase transition in a). Both the activation of the ferroelectric soft mode and the splitting of the AFD soft mode are strong indicators of a ferroelectric phase transition. Reprinted figure with permission from[38]. Copyright 2005 by the American Physical Society. 
component is a sign that tetragonality in the AFD phase of this ceramic sample is unusually low. The authors discuss possible sources for this reduced tetragonality, and suggest that it may be caused by a clamping effect of grain boundaries.

In this sample, $\mathrm{TO}_{2}$ and $\mathrm{TO}_{4}$ polar hard modes are seen well above $0 \mathrm{~K}$. $\mathrm{TO}_{2}$ in particular is weakly visible even at room temperature. These modes gradually increase in intensity as temperature is lowered. The authors of this paper show the temperature dependence with an exponentially increasing-type line to guide the eyes. I think it is more likely that what is observed is the lower half of what would a similar S-shaped curve to the Ca-substituted sample, if not for the limit of zero temperature. Certainly, it would not make physical sense for this intensity to increase without bound. In contrast to the $0.7 \%$ calcium substituted sample, the temperature response takes place over a much wider temperature range - there is a significant change over at least $100 \mathrm{~K}$ - and assuming that the same saturation pattern would be observed, that $100 \mathrm{~K}$ range accounts for only up to half of the temperature range that would be needed for saturation. Fig. 2.19 is reproduced from [36] and shows the Raman spectra recorded from these samples.

Dielectric measurements have been made on pure ceramic STO, and the behaviour is similar to single crystal STO[22]. No peaks are detected, Curie-Weiss type behaviour is seen at high temperatures, and is followed by a deviation from Curie-Weiss resulting in a saturation of permittivity below $20 \mathrm{~K}$. No frequency dispersion is detected, qualitatively similar behaviour to the pure STO crystal case. Signs of a frustrated ferroelectric transition are shown, but the samples cannot be said to enter a ferroelectric phase, even though the $\mathrm{TO}_{2}$ and $\mathrm{TO}_{4}$ hard modes are clearly seen in the Raman spectrum. As we have learned from our previous studies of BNT-BT, the dielectric measurements on their own are not necessarily a signal that the crystal structure has followed the same pattern. However, one of two things in the Raman spectrum gives us confidence in the lack of ferroelectricity. The authors assign one mode, labeled ' $\mathrm{X}$ ' in their figure as the $\mathrm{E}_{g}$ component of the AFD soft mode. If they are correct, then we can see that the $\mathrm{E}_{g} \mathrm{AFD}$ soft mode has not split into two components, and this is sufficient to show that the structural symmetry has not fallen into orthorhombic. If they are incorrect in this assignment, then this is most likely the ferroelectric soft mode, which shows a hardening compared to the single crystal case. This has been seen before in polycrystalline samples. The fact that this mode is hardened, and that it has not 


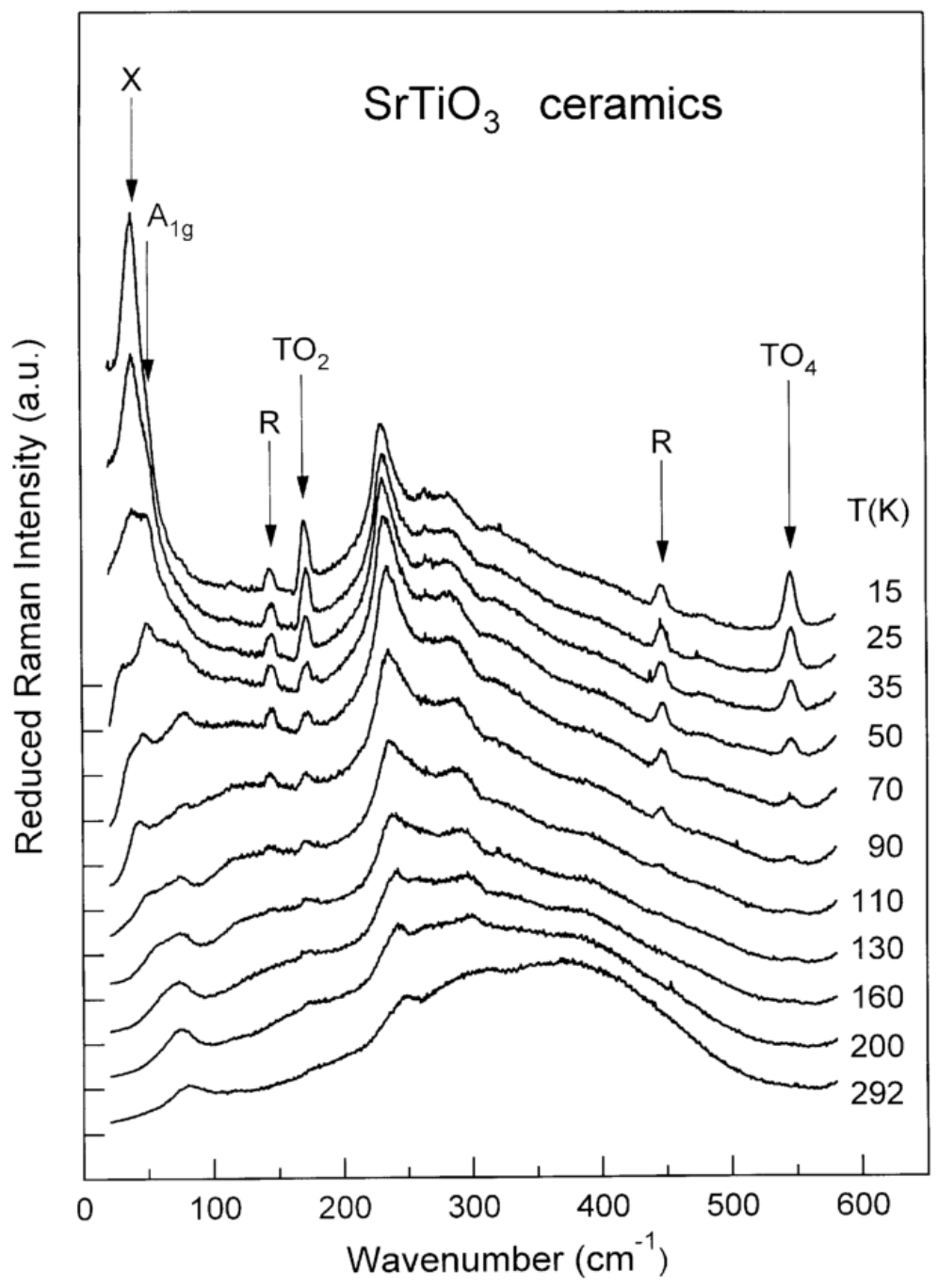

Figure 2.19: Temperature dependent Raman spectra of STO ceramics. The spectra have been vertically offset for ease of viewing. The R-point AFD modes can be seen, as can the $\mathrm{TO}_{2}$ and $\mathrm{TO}_{4}$ first-order modes. The intense mode marked $\mathrm{X}$ is thought to be either a component of the AFD soft mode, or the ferroelectric soft mode. Figure reproduced with permission from [36]. 
split into components is also a direct Raman feature that supports the lack of a ferroelectric phase, so whatever the interpretation of this feature it suggests that there is no further phase transition after the AFD phase transition on cooling.

The authors interpret the presence of the Raman-silent polar modes in the Raman spectrum in the paraelectric phase thus: Near grain boundaries in this ceramic, there exists a surface layer of frozen polarisation. This polarisation can cause local symmetry breaking regions, in which the polar first order phonons become Raman active. The slow increase in intensity with temperature then corresponds to the usual increase in the volume of the local symmetry breaking regions. Because the polarisation is 'frozen in,' and not free to rotate, these grain boundaries do not enhance the dielectric permittivity of the material like polar phase volumes of STO usually do.

The 2007 study[22] also compared samples with low concentrations of grain boundaries (large-grained ceramics) to samples with high grain boundary concentrations (fine-grained ceramics). The results of this research showed very clearly that the fine-grained ceramics had more pronounced loss of dielectric permittivity, greater soft mode hardening and more intense first-order Raman scattering. This was good evidence that the grain boundaries were having these effects, and were undesirable in the growth of technologically useful STO.

So this, then, is a material which does not undergo a ferroelectric phase transition, as shown by permittivity measurements. It does not undergo an associated tetragonal to orthorhombic structural phase transition either. And yet the polar hard modes $\mathrm{TO}_{2}$ and $\mathrm{TO}_{4}$ are Raman activated, albeit with noticeably different temperature dependence to ferroelectric STO. The presence of $\mathrm{TO}_{2}$ and $\mathrm{TO}_{4}$ polar hard modes in STO samples cannot therefore be used on their own as indicators of ferroelectricity - they have shown themselves to be active both in ferroelectric phases, and non-ferroelectric phases. The temperature dependence of these mode intensities is of more importance in interpreting these peaks to determine the nature of the crystal. With rapid rises in intensity with respect to temperature, one can be more confident of ferroelectric properties. If the rate of intensity increase is slow, then ferroelectricity is likely not present in the sample. 
Case study 4: Single crystal strain-free thin films

The studies described in this section are the work of Jang et al.[42]. These samples were grown by pulsed laser deposition. Starting from a single crystal STO [001] substrate, a $50 \mathrm{~nm}$ strontium ruthanate layer was grown as a bottom electrode, then the STO film was grown on top of this. Temperature dependent Raman spectra are shown for this sample. The first thing of note in this sample is that there are no features in these spectra that can be assigned to the AFD soft mode or the AFD $450 \mathrm{~cm}^{-1}$ hard mode. There is a feature that corresponds to the AFD $150 \mathrm{~cm}^{-1}$ hard mode, but it is just barely visible. The lack of features/weakness of features associated with the AFD transition is a trait common to many thin-film STO systems, as well as other nano-structured STO samples, and will be discussed in case study 5 (single crystal non-stoichiometric strain-free films).

(Note - one should for sure expect to see the AFD hard modes from the substrate at least, which is pure STO. However, the lower electrode layer of SRO acts as a mirror, meaning that the Raman does not probe the substrate in this case.)

This sample has multiple first-order peaks from the polar phonons visible at room temperature. $\mathrm{TO}_{2}, \mathrm{LO}_{3}$, and $\mathrm{LO}_{4}$ can all be seen at $300 \mathrm{~K}$, and $\mathrm{TO}_{4}$ can be seen as well below $250 \mathrm{~K}$. The peaks all increase in intensity as temperature decreases. At $10 \mathrm{~K}$ an additional peak is visible at $50 \mathrm{~cm}^{-1}$, which the authors assign as $\mathrm{TO}_{1}$, the ferroelectric soft mode. Unfortunately, the authors of this study did not present spectra between $50 \mathrm{~K}$ and $10 \mathrm{~K}$. Without observing the temperature dependence of the mode, it is difficult to properly assess that assignment. However, the absence of this peak at higher temperatures does lend it some credence. The temperature dependence might also show hardening or softening of this mode, which would tell us more about the lattice dynamics. The high frequency of this peak compared to the pure STO $\mathrm{TO}_{1}$ frequency suggests that some effect is hardening the mode, usually an indicator of suppression of ferroelectric properties. However, as the temperature dependence of this peak is not shown it is difficult to have much confidence in this.

Dielectric measurements show the material to not undergo a ferroelectric tran- 

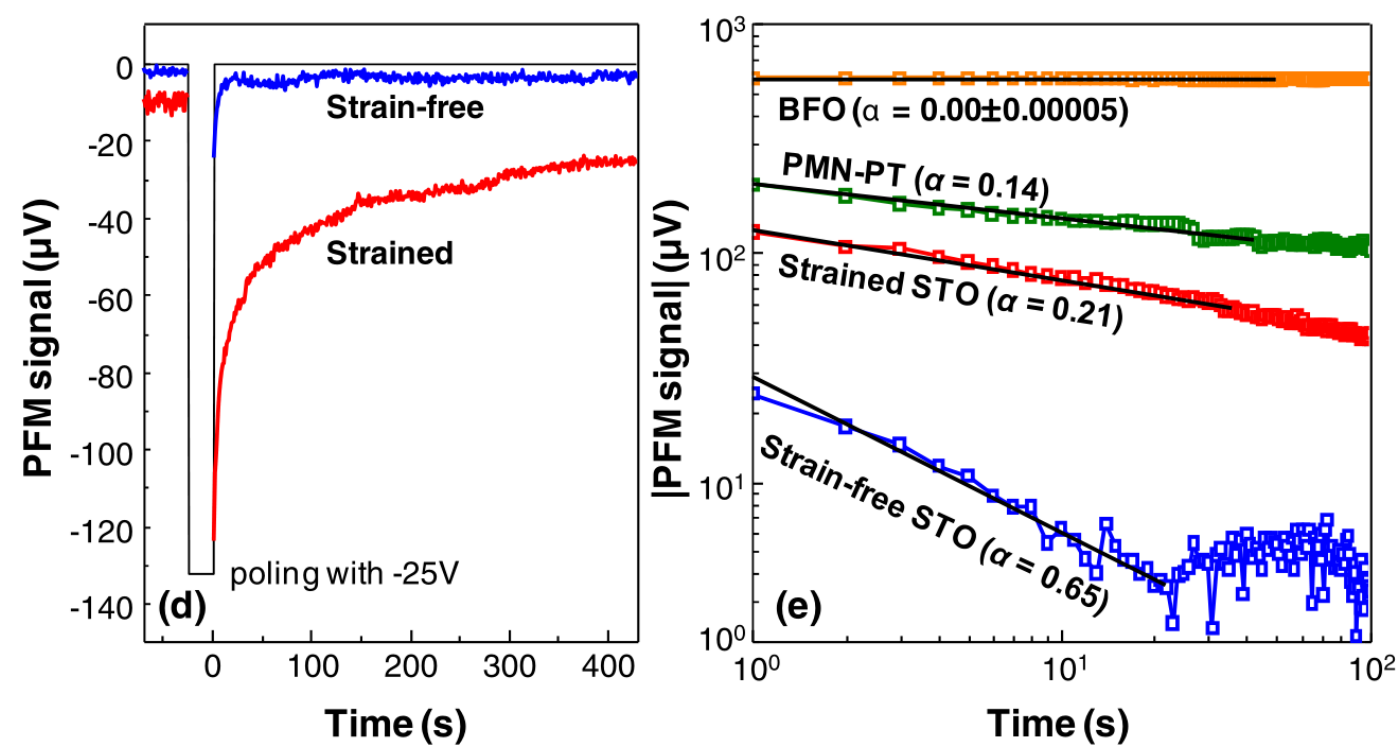

Figure 2.20: Figure showing the decay over time of the piezoelectric force microscopy signal from STO, comparison of strain and strain-free. Reprinted figure with permission from[42]. Copyright 2005 by the American Physical Society.

sition. The permittivity shows the usual STO pattern of Curie-Weiss behaviour at higher temperature followed by a saturation to a constant value at near zero temperature. Piezoelectric force measurements done on this sample do show a very small hysteresis loop however. This hysteresis loop rapidly decays in intensity over time. The decay shows the ferroelectricity displayed by this sample to be relaxor dominated, rather than displacive. Even then, the effect is weak. The intensity is almost an order of magnitude less, and in addition the time constant for the decay is much shorter than other relaxor ferroelectrics. Fig. 2.20 is reproduced from [42], it shows the magnitude of polarisation hysteresis loops over time. The low intensity and short decay can be seen in this figure.

The authors of this paper propose that the appearance of the first order Raman modes may be induced by impurities in the thin film caused by a small amount of Sr-Ti non-stoichiometry (and corresponding oxygen vacancies, or interstitials). This explanation is based upon the similarity of the Raman spectra of their sample to intentionally Sr-deficient STO thin films. The similarity between the Raman spectra is very strong. Other than some differences in the peak around $90 \mathrm{~cm}^{-1}$, the features are identical, and the relative intensities almost the same (the polar features are slightly weaker in the film studied in this paper than in the intention- 
ally Sr-deficient film, by about 15-20\%).

So the STO/SRO/STO films grown in this paper are an interesting case. They do show ferroelectricity, but it is very weak, almost to the point of insignificance. One possible explanation is that the piezoelectricity measured in these samples come from local polar micro-regions, making up a small volume fraction of the whole film. The spectral features of STO visible in this sample are polar hard modes $\mathrm{TO}_{2}, \mathrm{LO}_{3}, \mathrm{TO}_{4}$ and $\mathrm{LO}_{4}$ with a temperature dependence showing variation in intensity over about $200 \mathrm{~K}$, and a mode assigned to $\mathrm{TO}_{1}$. The mode assigned as $\mathrm{TO}_{1}$ in this paper occurs at a much higher frequency than that of strongly ferroelectric STO samples, which indicates a suppression of ferroelectricity.

Case study 5: Single crystal non-stoichiometric strain-free films

The studies described in this section are the work of Tenne et al.[50]. This paper is of particular interest, as the researchers used the same method of UV Raman spectroscopy that I used to study the samples in the STO section of this thesis. These samples were $100 \mathrm{~nm}$ thick films, grown by molecular beam epitaxy directly on Ti-terminated STO[001] substrates. The samples were grown with intentionally altered Sr-Ti ratios to measure the effect that this non-stoichiometry has on the phonon modes of STO thin films. This study was done using UV Raman spectroscopy, so one can be confident that the pure STO substrate is contributing negligible amounts to the observed Raman spectra, even though there is no intervening reflective layer. Fig. 2.21 is reproduced from the paper. It shows Raman spectra of the non-stoichiometric strain-free STO thin films.

One of the films grown was stoichiometric to within uncertainties (measured as $x=1.02 \pm 0.4$ by Rutherford backscattering, where $x$ is the Sr stoichiometry). The Raman spectrum of this sample at $10 \mathrm{~K}$ does show clearly the AFD $\mathrm{A}_{1}$ component of the AFD soft mode at $50 \mathrm{~cm}^{-1}$, and shows weakly the AFD hard mode at $145 \mathrm{~cm}^{-1}$. The AFD hard mode at $450 \mathrm{~cm}^{-1}$ is not seen in this spectrum. In addition, the $\mathrm{FE}$ hard modes $\mathrm{TO}_{2}$ and $\mathrm{LO}_{4}$ are both visible, though very weak. No other FE hard modes are visible.

The spectra of the Sr-deficient samples do not show any AFD peaks. One 


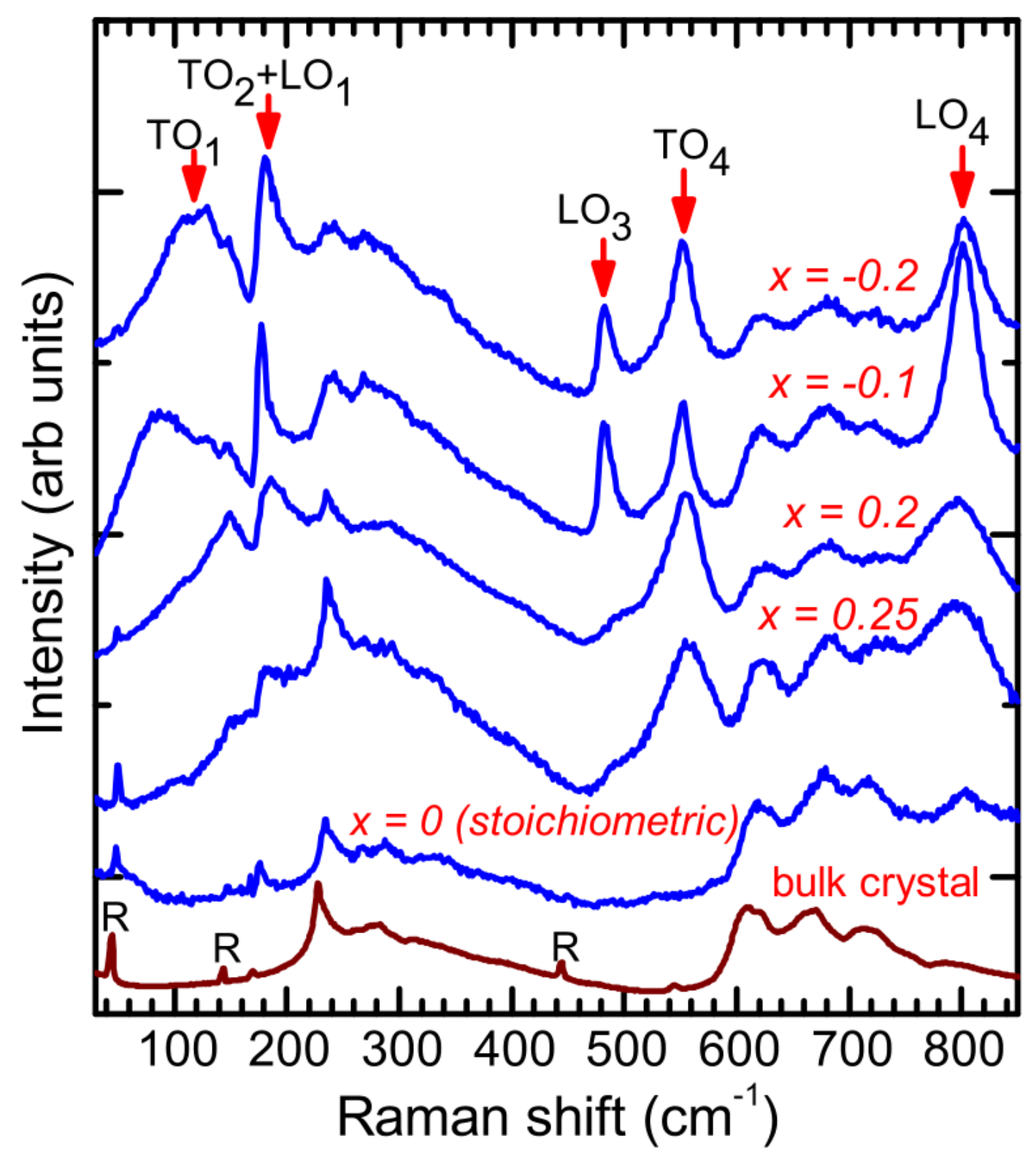

Figure 2.21: Raman spectra of non-stoichiometric single-crystal thin films of STO. The spectra have been vertically offset for ease of viewing. $x$ is the Sr excess (or deficiency, for negative values). Spectra were taken at $10 \mathrm{~K}$, and non-stoichiometric samples show first-order modes. Additionally, there is a marked difference in the AFD modes between Sr-deficient and Sr-excess films. Figure reproduced with permission from [50]. 
could possibly convince one's self that there is a feature at $50 \mathrm{~cm}^{-1}$ in some of the spectra that is the $\mathrm{A}_{1 g}$ component of the AFD soft mode, but it is not present in every spectrum. For certain the intensity is at least ten times smaller than in the stoichiometric case. The $\mathrm{TO}_{2}, \mathrm{LO}_{3}, \mathrm{TO}_{4}$ and $\mathrm{LO}_{4}$ hard modes are all strong and clear, with the $\mathrm{TO}_{2}$ mode showing Fano asymmetry, suggesting that local polar symmetry-breaking regions are present. The Sr-deficient samples also have a peak near $90-110 \mathrm{~cm}^{-1}$ (depending on $\mathrm{Sr}$ content), which they assign as $\mathrm{TO}_{1}$. As with the previous example, temperature dependence is not shown over the crucial range, so it is difficult to assess whether this is the ferroelectric soft mode or not. $\mathrm{TO}_{3}$ is not seen in this sample.

The Sr-rich samples do show the same AFD modes as were seen in the stoichiometric film; they is almost as strong as in the stoichiometric film. The same four polar hard modes are also present. However, $\mathrm{TO}_{2}$ and $\mathrm{LO}_{3}$ are both much weaker than in Sr-deficient films, and all four peaks are much broader. $\mathrm{The}^{\mathrm{TO}_{2}}$ peak is still strongly asymmetric.

Between the two types of stoichiometry, there is a very clear contrast in the behaviour of the AFD modes. Strontium vacancies suppress these modes strongly, while excess strontium suppresses them only weakly, resulting in them still appearing in the Raman spectra. The reasons for this are not discussed, but Sr-vacancies offer a possible explanation for the lack of these modes in Raman spectra of other thin films in existing in the literature.

The temperature dependence of the non-stoichiometric films was measured, and the by now familiar pattern of slowly decreasing polar hard mode intensity with rising temperature was observed. The width of the S-shaped curve they depict is over $300 \mathrm{~K}$, with the intensity not appearing to saturate at low temperatures. In the case of Sr-rich films, the modes stay Raman active up above $400 \mathrm{~K}$, the highest temperature shown. The temperature dependence of the AFD modes (where seen) is not well shown by the figures presented. However, they show no reason to doubt that the normal AFD phase transition occurs somewhere between 100 and $150 \mathrm{~K}$. The AFD lines are absent above this temperature.

The authors do not present electric measurements, but say that the near-exact match of their spectra to previously measured STO thin films (the samples in 
case study 4) shows their films to have the same properties as those ones. On this basis they claim them to be ferroelectric, but see the above case study for my reservations about the weakness of the ferroelectric effect in these samples. The electric properties of the Sr-rich films are not discussed at all. However, a later paper made these measurements on non-stoichiometric STO[51]. It must be noted that the samples in that study were bulk polycrystalline, and not thin film. However, clear indication of very weak ferroelectricity $\left(P_{r}=0.004 \mu \mathrm{C} / \mathrm{cm}^{2}\right)$ is seen in those samples.

It can be seen from these samples that STO is quite sensitive to the stoichiometry, with Sr-Ti imbalances able to induce local symmetry breaking regions. It also shows us that Sr-Ti imbalances can very easily be introduced to samples if great care is not taken in the deposition process. It shows us that one sign of Sr-vacancies may be a lack of AFD modes in STO samples. And finally, it shows us spectra that we know not to be associated with any significant ferroelectricity in STO: Polar TO and LO hard modes with weak temperature dependence (although they have relatively narrow widths), and a broad peak around $100 \mathrm{~cm}^{-1}$ which is possibly the $\mathrm{TO}_{1}$ ferroelectric soft mode showing a hardening compared with pure single crystal STO.

Case study 6: Single crystal strained films

There are a number of examples of thin strained STO films which have been shown to display ferroelectric properties, sometimes even up to room temperature. Unfortunately, almost without exception no Raman spectra of these samples have been published. The first results of room temperature ferroelectricity came from Haeni et al.[1], showing electrical measurements made on $50 \mathrm{~nm}$ STO grown by molecular beam epitaxy on the [110] surface of $\mathrm{DyScO}_{3}$. In the same study, $50 \mathrm{~nm}$ STO grown by molecular beam epitaxy on $[001]\left(\mathrm{LaAlO}_{3}\right)_{0.29} \times\left(\mathrm{SrAl}_{0.5} \mathrm{Ta}_{0.5} \mathrm{O}_{3}\right)_{0.71}$ (LSAT) was measured. A strong peak in dielectric permittivity was found at just below $300 \mathrm{~K}$ for the STO/DSO. No peak was found for STO/LSAT. Similarly, another group found a ferroelectric permittivity peak in $60 \mathrm{~nm}$ STO grown on [110] $\mathrm{NdGaO}_{3}$ (NGO)[42]. The dielectric peak found in this case was far broader and weaker than the STO/DSO peak, and the transition temperature was $110 \mathrm{~K}$, 


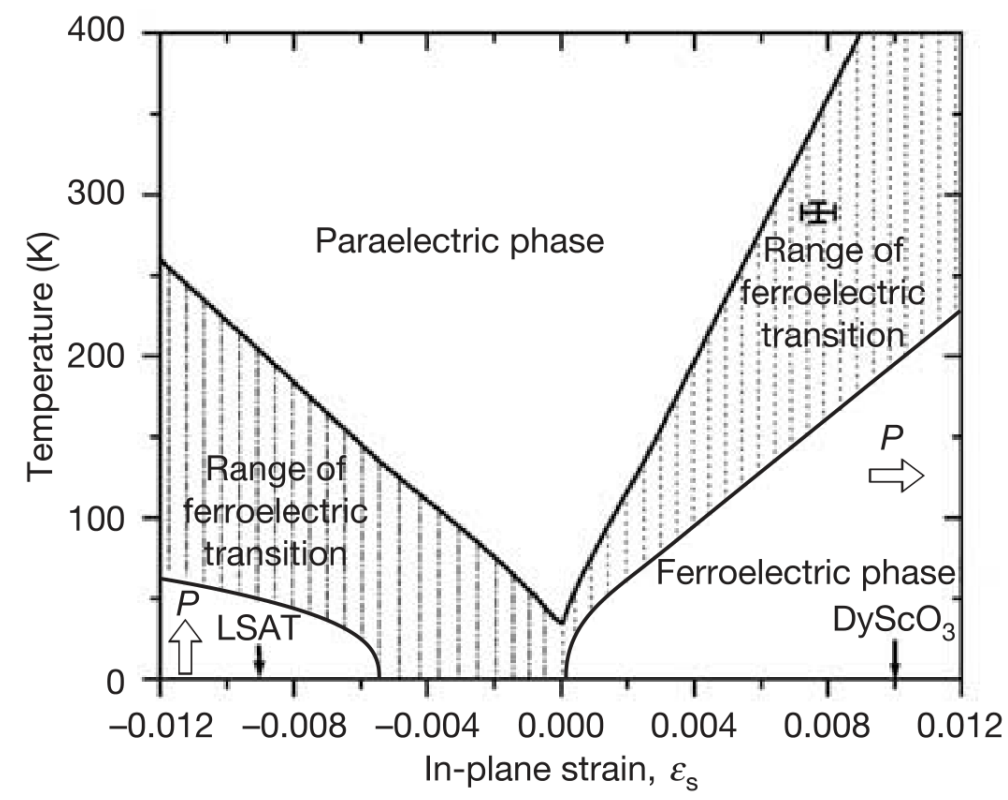

Figure 2.22: Temperature-strain phase diagram of STO. The graph was created from thermodynamic analysis. The range of transition is due to conflicting reports on parameters used in the modeling. Reprinted by permission from Macmillan Publishers Ltd: Nature[1], copyright 2004.

well below room temperature. DSO has a lattice mismatch of $+1 \%$ with STO (tensile), and NGO has a mismatch of $-1.2 \%$ (compressive).

There is a body of theoretical work done on STO. The Haeni paper presents a figure showing the expected electric phase of epitaxially grown STO[100] under a given strain condition, based on thermodynamic analysis. That figure is reproduced here, in Fig. 2.22. Both compressive and tensile biaxial strain can induce a ferroelectric phase in STO, provided that the strain is large enough. Larger compressive strains are required to produce the same increase in Curie temperature as smaller tensile strains. The predicted phase transition temperatures for biaxially strained STO depend on the precise values of elastic and electrostrictive properties of STO, over which there is some variation in experimental results. However, both the STO/DSO and STO/LAO measured transitions are within the range predicted by this theory.

Another group grew STO films on LAO samples - though this was not true epitaxy because an intermediate SRO layer was grown, and the films were much thicker at $500 \mathrm{~nm}$ or more[52]. At $5 \mathrm{~K}$ the AFD hard and soft modes are not 
visible. $\mathrm{TO}_{2}$ is present and shows the usual Fano asymmetry. $\mathrm{TO}_{4}$ is also present, as is $\mathrm{TO}_{3}$, and there is a broad peak near $100 \mathrm{~cm}^{-1}$, similar to that seen in the previous case studies. The temperature dependence of the $\mathrm{TO}_{4}$ and $\mathrm{TO}_{2}$ (but not $\mathrm{TO}_{3}$ ) modes is shown, and it is highly unusual. It exhibits not a gradual rise, but a rise with a peak in the middle of it, at $85 \mathrm{~K}$. The modes reach their maximum intensity at around $50 \mathrm{~K}$, after beginning the rise at around $100 \mathrm{~K}$. This punctuated S-shape curve is broader than the Ca-substituted single crystal, but narrower than non-stoichiometric thin films, and other samples that do not undergo a ferroelectric phase transition. The authors do not offer an explanation for the peak at $85 \mathrm{~K}$, but they do note the similarity between the imaginary part of the dielectric function and the measured polar hard mode temperature dependence. Fig 2.23 is reproduced from [52] and shows the dielectric function and first-order mode intensity in these films.

Dielectric measurements were done also on this sample, and there is a ferroelectric anomaly-like peak in the permittivity for this sample, occurring at $35 \mathrm{~K}$, the whole peak very broad. The value of peak is a little under $\epsilon_{r}=1000$, much smaller than the peak measured in Haeni et al.'s STO/DSO film of just under 7000. This would seem to indicate that the STO does go into a ferroelectric phase at around $35 \mathrm{~K}$, and that the ferroelectricity was fairly weak. Low frequency Raman spectra are not provided for this sample, so we have no way of knowing if the ferroelectric soft mode appears, or if there is any splitting of the $\mathrm{E}_{g}$ AFD soft mode component. Likewise, temperature dependence was not displayed for the $\mathrm{TO}_{3}$ hard mode, so one cannot tell if its appearance or a peak in its intensity coincides with the peak in the permittivity - or with the peak in polar hard mode intensity at $85 \mathrm{~K}$. The differences between this Raman spectrum, and that of other materials with no permittivity peak are: the appearance of the $\mathrm{TO}_{3}$ mode at low temperatures, and the temperature dependence of the $\mathrm{TO}_{2}$ and $\mathrm{TO}_{4}$ modes including a comparatively rapid rise in line strengths, a saturation above $0 \mathrm{~K}$, and the interesting extra peak.

Single crystal stained thin films then, in summary, are capable of undergoing a ferroelectric phase transition as shown by a peak in the dielectric permittivity - so long as the degree of lattice strain is sufficiently high. Both theoretical and experimental studies have shown this. There is a dearth of Raman spectra of such samples due to experimental difficulties, so presentation of these results would be valuable to the understanding of the interaction between lattice dynamics and 


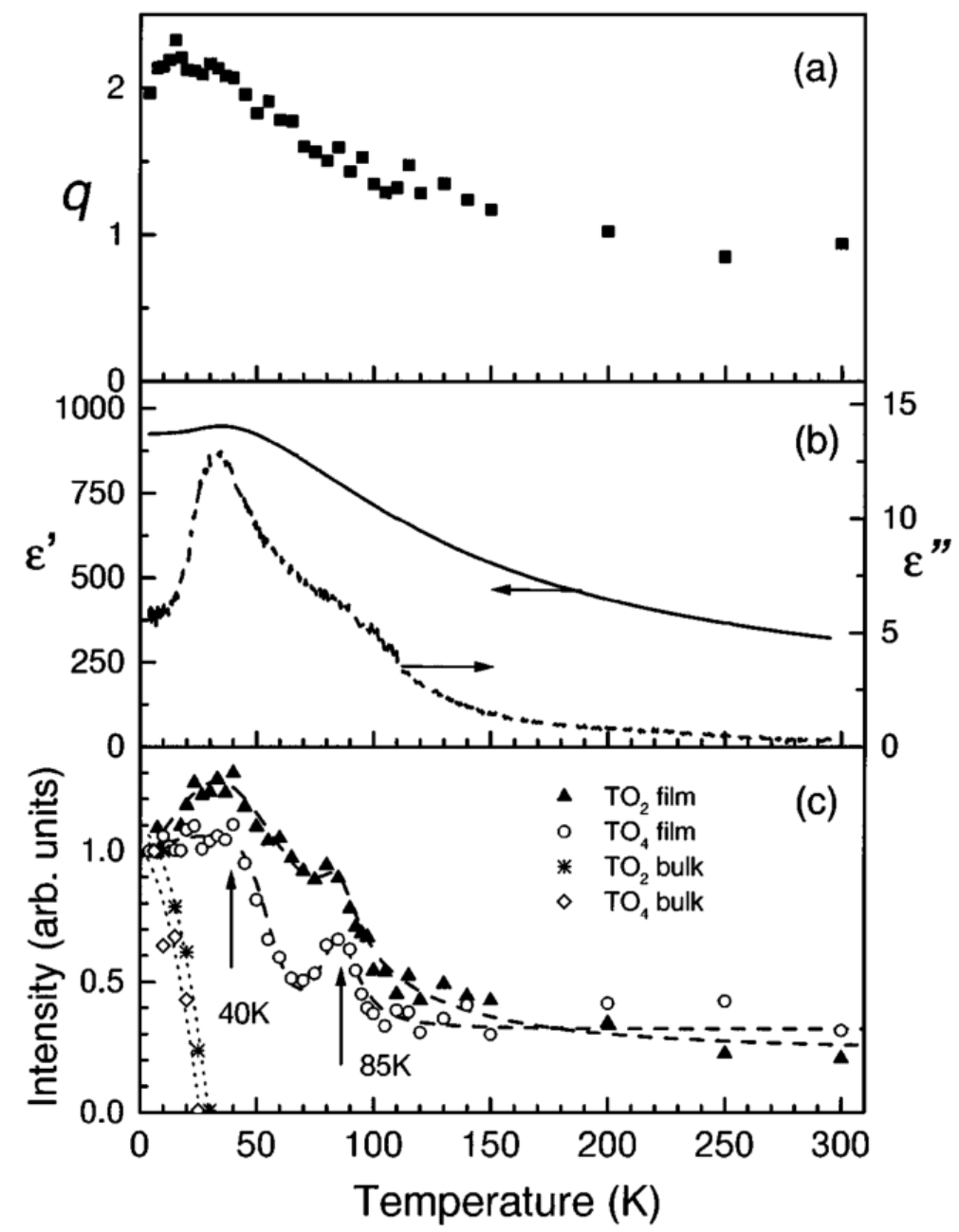

Figure 2.23: Figure showing temperature dependence of several factors. Upper: asymmetry factor, $q$, of $\mathrm{TO}_{2}$ mode in their spectra. Middle: Dielectric permittivity, showing a very slight ferroelectric-type peak. Lower: first-order mode intensity, compared with bulk. Reprinted figure with permission from[38]. Copyright 2005 by the American Physical Society. 
ferroelectricity in STO and other ferroelectrics.

Case study 7: Polycrystalline thin films

Thin films can also be grown in polycrystalline, rather than epitaxial structures. The studies described in this section are the work of Du et al.[40]. These samples were grown to $260 \mathrm{~nm}$ in thickness on top of quartz substrates, and shown to be polycrystalline by XRD (grains crystallised in random directions). Raman spectra of these samples were taken only down to $95 \mathrm{~K}$. The low temperature spectra show $\mathrm{TO}_{2}, \mathrm{TO}_{3}, \mathrm{TO}_{4}$, and $\mathrm{LO} 2, \mathrm{LO}_{4}$ polar hard modes, and a broad peak around $100 \mathrm{~cm}^{-1}$ which is likely the ferroelectric soft mode in addition to the second order STO features. The $\mathrm{TO}_{2}$ mode is strongly asymmetrical, to a higher degree than other thin film samples. This is possibly related to the grain boundaries in the film, possessing a greater range of continuous disordered states for the $\mathrm{TO}_{2}$ mode to interact with. The temperature dependence of the polar modes is shown, and the result is relatively steady intensities of $\mathrm{TO}_{2}$ and $\mathrm{TO}_{4}$ from room temperature down to $130 \mathrm{~K}$. Below $120 \mathrm{~K}$, a rapid rise in intensity begins. Unfortunately, the paper only reported spectra down to liquid nitrogen temperature, so the low temperature and possible saturation behaviour are unknown. The long period of constant intensity is quite different from many other samples studies, and it too may have to do with the grain boundaries. Polar modes may be active in these regions due to increased defects, decreased stoichiometry or relaxation of long-range symmetry rules. As these boundary regions persist to high temperatures, the modes therefore persist in these regions too even though there is no signal from the grain bulk.

Electrical measurements are not available for this sample at time of writing, unfortunately. Studies of a much thicker $(1 \mu \mathrm{m})$ polycrystalline film have been done[29]. They measured a Curie-Weiss behaviour followed by saturation, with no peak developing down to $15 \mathrm{~K}$. The film used by this group was grown on a different substrate (STO) in addition to being much thicker, so any potential strain effects in the Du film will not be expressed in those measurements. However, due to the non-epitaxial nature of both films, it is unlikely that lattice mismatch will have a strong effect. 
The important takeaways from these examples are the grain boundary effect causing first order Raman lines to persist up to room temperature and beyond, and also the appearance of $\mathrm{TO}_{3}$ in a sample which is not ferroelectric.

Case study 8: Oxygen reduced STO single crystals

The studies described in this section are the work of Tenne et al.[53]. The samples in this case are single crystals of pure STO which have been annealed in a reducing environment at high temperatures for various lengths of time to produce oxygen vacancies in the crystal. The oxygen vacancies make these materials conducting, and therefore not ferroelectric. But seeing as the stoichiometry of STO samples in literature is often in question, it is of value to see the Raman spectra of STO which we know to have significant oxygen vacancies for comparison's sake. Fig. 2.24 is reproduced from this paper, and shows the Raman spectra of the STO crystal in various states of reduction, all at $10 \mathrm{~K}$.

The as prepared, not-reduced sample is not completely pure, as very weak $\mathrm{TO}_{2}$ and $\mathrm{TO}_{4}$ peaks can be seen in it. The AFD features and second-order features are visible, the same as in pure STO. As oxygen vacancies are gradually added to STO, the first noticeable change in the Raman spectrum is the appearance of a broad peak at $135 \mathrm{~cm}^{-1}$. This occurs at a level of oxygen vacancies that gives resistivity of $135 \mathrm{~m} \Omega \mathrm{cm}$. When the oxygen vacancy level is further increased, a noticeable increase in the intensity of $\mathrm{TO}_{2}$ and $\mathrm{TO}_{4}$ polar hard modes is observed. This starts at a resistivity level of $15 \mathrm{~m} \Omega \mathrm{cm}$. Simultaneously, additional modes appear; a doublet mode at $500 \mathrm{~cm}^{-1}$ and a sharp narrow peak at $700 \mathrm{~cm}^{-1}$. Both are weak, but visible in the spectra. Finally, the intensity of the new modes increases significantly. Resistivity is now $13 \mathrm{~m} \Omega \mathrm{cm}$. All of these changes to the spectrum are completely reversible by re-oxygenating the sample: upon annealing in a furnace with flowing ultra pure oxygen, the original Raman spectrum is recovered. Although the polar features appear at low oxygen concentrations, there is no splitting in the $50 \mathrm{~cm}^{-1}$ AFD soft mode, so we can rule out a lowering of crystal symmetry to an orthorhombic, and therefore ferroelectric phase.

In another investigation, STO substrates were reduced, and the optical con- 


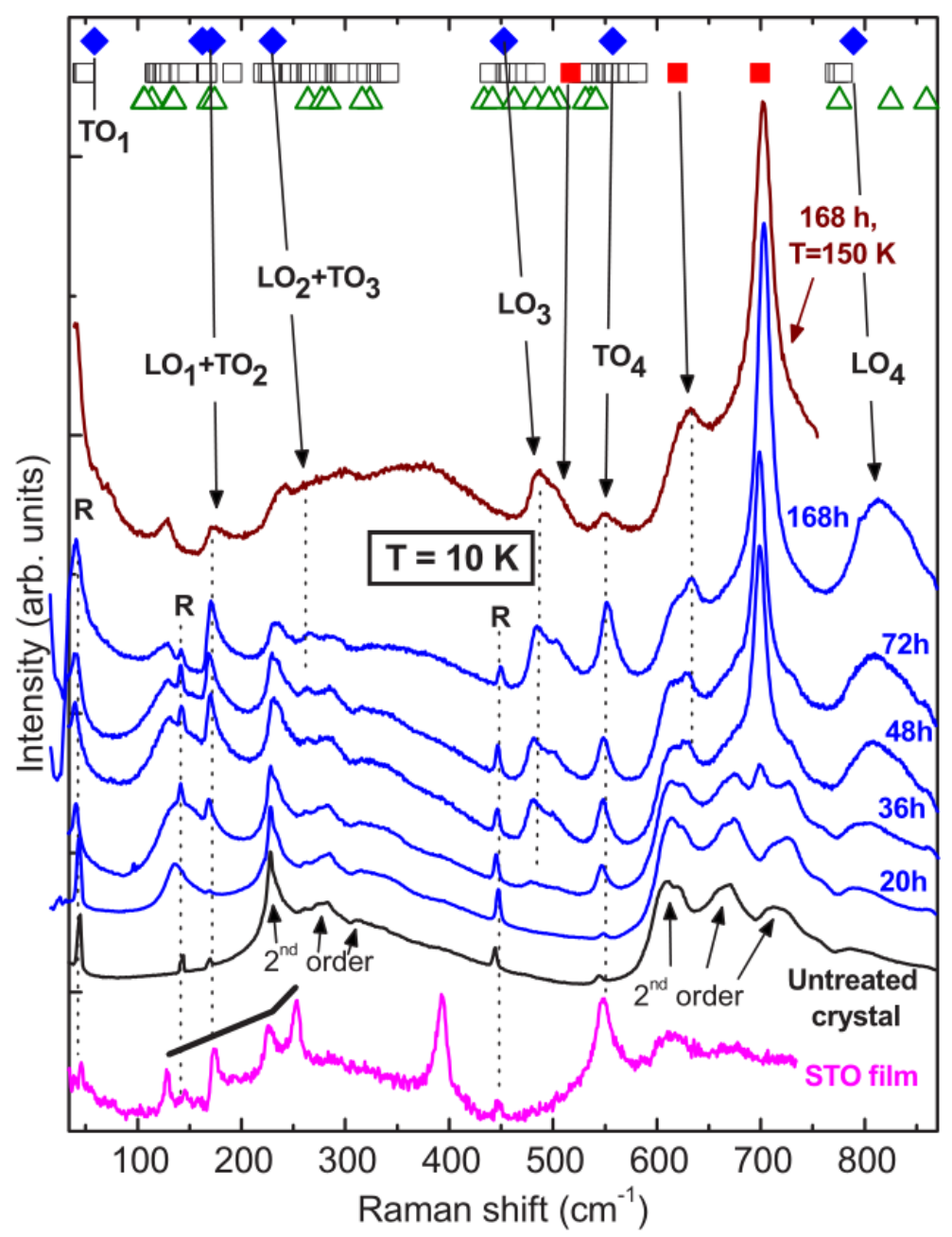

Figure 2.24: Raman spectra of STO with various levels of oxygen vacancies, all taken at $10 \mathrm{~K}$. The spectra have been vertically offset for ease of viewing. The spectra are identified by the amount of time spend in a reducing atmosphere. Reprinted figure with permission from[53]. Copyright 2007 by the American Physical Society. 
ductivity measured with respect to frequency to find the effect of the oxygen vacancies on the optical modes[45]. In this measurement technique, the $\mathrm{TO}_{1}$ mode can be seen, unlike in Raman where it is silent. The measurements in this study are not temperature dependent, and we have only room-temperature information. Nonetheless, the spectra do show that there is a shift to higher frequency of the $\mathrm{TO}_{1}$ mode with increasing oxygen vacancy concentration. Also shown is the same information for Nb-doped STO. Here the carrier concentration is considerably larger than in the reduced STO substrates, and the $\mathrm{TO}_{1}$ mode can be seen to have shifted significantly higher in frequency - from about $90 \mathrm{~cm}^{-1}$ in the pure STO case to about $180 \mathrm{~cm}^{-1}$. The $\mathrm{TO}_{2}$ and $\mathrm{TO}_{4}$ modes have shifted slightly higher in this sample also. This dramatic hardening of the $\mathrm{TO}_{1}$ mode warrants some reflection. As the dielectric to ferroelectric (corresponding to tetragonal to orthorhombic) phase transition is driven by the freezing of this soft mode, a hardening such as this would be expected to have the effect of lowering the phase transition temperature. No data is presented for temperature dependent measurements of oxygen-reduced STO, so we cannot be certain, but the results shown do imply that oxygen vacancies will suppress ferroelectricity in STO, and more generally that current carriers will suppress ferroelectricity in STO. This is not surprising of course, as conduction will tend to inhibit ferroelectricity.

In another study, nominally unstrained STO films (grown on STO/SRO heterostructures) that were grown with lowered oxygen partial pressures were found to be weakly ferroelectric, with remnant polarisation of $1.5 \mu \mathrm{C} / \mathrm{cm}^{2}$ at $100 \mathrm{~K}[54]$ (compare this with $8 \mu \mathrm{C} / \mathrm{cm}^{2}$ at $100 \mathrm{~K}$ in the strained STO/DSO film[32]). The authors of that paper did not think that oxygen vacancies alone could be responsible for the ferroelectricity, and later published an explanation based on linked O-O-Sr vacancy clusters - these clusters carry a defect dipole moment that can express ferroelectricity. The ferroelectricity was not, they suggest coming from the STO, but from these defects[55]. Significantly, the films were found by XRD to be in the tetragonal phase, and not the orthorhombic phase where ferroelectricity is normally found in STO.

In summary, these studies show us the effect in the Raman spectrum of oxygen vacancies, and the effect of oxygen vacancies on the ferroelectric soft mode. The result is that oxygen vacancies are not on their own able to induce a phase transition from the tetragonal to the orthorhombic, ferroelectric phase. They can, 
however, activate first order Raman modes in STO. The oxygen vacancies also create new lines in the Raman spectrum simultaneously with activating the firstorder STO lines, so one can tell whether the first order scattering is caused by oxygen vacancies or not. This can help us in identifying whether oxygen vacancies may be having an influence on the samples of STO we study.

Case study 9: $\mathrm{K}_{1-x} \mathrm{Li}_{x} \mathrm{TaO}_{3}$ crystals

The studies described in this section are the work of Toulouse et al.[2]. This example is not of STO, but of other ferroelectric perovskite materials, including $\mathrm{K}_{1-x} \mathrm{Li}_{x} \mathrm{TaO}_{3}$ (KLT). Many papers writing on STO make arguments based on the $\mathrm{TO}_{3}$ mode, and the literature trail leads back to this paper. The mode is much more clearly seen, and more easily studied in KLT than in STO. It is therefore worthwhile to study its behaviour in this system to aid in the interpretation of the $\mathrm{TO}_{3}$ peak in STO. The comparison is not so bad, as both STO and KLT are perovskite oxides, and the phase transitions in KLT are very similar to those in STO, and other (incipient) ferroelectrics. The crystals used in this study can be confirmed to be ferroelectric below $30 \mathrm{~K}$ by dielectric permittivity measurements. Fig. 2.25 shows the temperature dependence of the $\mathrm{TO}_{2}$ (polar) and $\mathrm{TO}_{3}$ (nonpolar) Raman line intensities in KLT. Note how the $\mathrm{TO}_{2}$ peak appears at higher temperature than $\mathrm{TO}_{3}$. The onset of $\mathrm{TO}_{2}$ intensity occurs simultaneously to the onset of remnant polarisation being detected. At lower temperature, the $\mathrm{TO}_{3}$ peak starts to emerge. As it does, there is a sudden drop in intensity of the $\mathrm{TO}_{2}$ mode. The temperature at which this occurs also corresponds to the temperature at which the dielectric function has its peak.

The explanation given by the authors of this paper, which has been accepted and carried forwards through the literature is that the $\mathrm{TO}_{3}\left(\mathrm{~F}_{2 u}\right)$ mode is optically silent in the cubic phase, appearing in neither IR or Raman measurements. This is a point of difference to the other hard modes $\mathrm{TO}_{2}, \mathrm{TO}_{4}$ and $\mathrm{LO}_{4}$ and to the soft mode $\mathrm{TO}_{1}$, which are all IR active (or 'polar'). Because of the polarity of those modes, they are able to couple to even very small local polar regions, and by this become Raman active. The appearance of $\mathrm{TO}_{2}, \mathrm{TO}_{4}, \mathrm{LO}_{4}$ therefore can be linked to the appearance of polar micro-regions in the material, even where a 


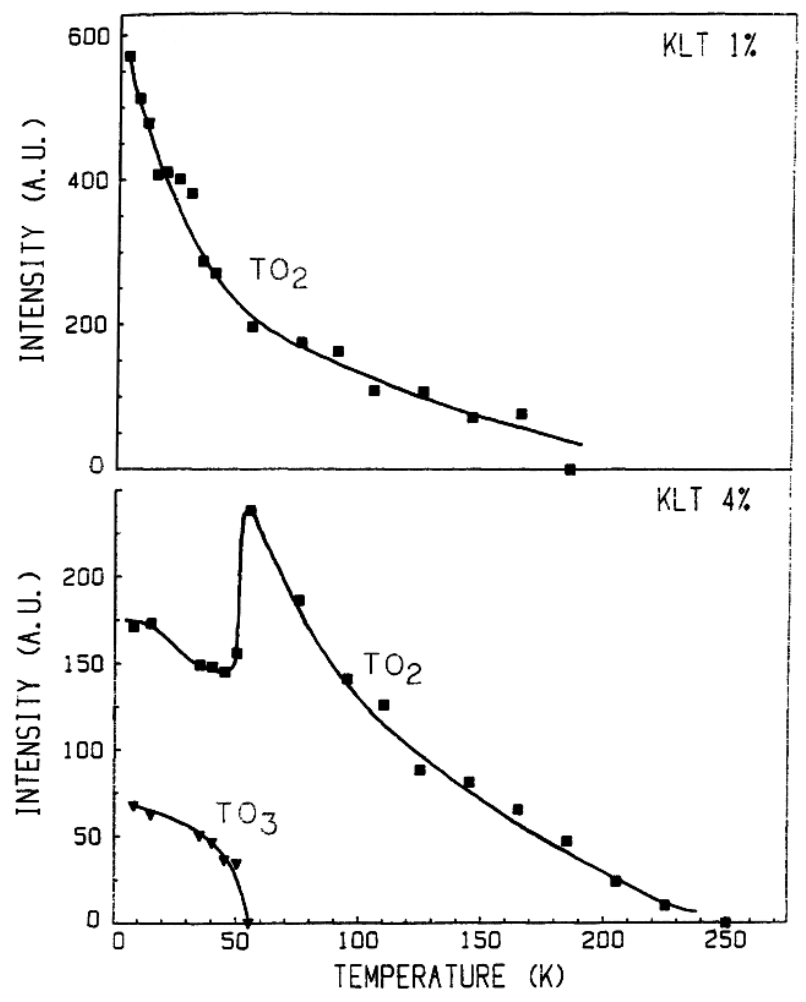

Figure 2.25: Temperature dependence of first-order scattering in KLT. Above: 1\% Li, Below: $4 \% \mathrm{Li}$. the $4 \% \mathrm{KLT}$ has a ferroelectric phase transition at about $50 \mathrm{~K}$, and one can see the spectral changes associated with it. $1 \% \mathrm{KLT}$ is not ferroelectric. Reprinted figure with permission from[2]. Copyright 1992 by the American Physical Society. 
phase transition throughout the material does not occur. $\mathrm{TO}_{3}$, as it does not have this access to polar activation, is a much better indicator of a phase transformation into a cohesive structure that lacks centrosymmetry. I am somewhat skeptical of this proposal. I am not aware of any mechanism whereby polarity of a mode can activate Raman scattering, a different process which depends on different features of the vibrations. Certainly, the authors propose no mechanism. However, this much can be unambiguously said: in the high temperature, high symmetry phase of KLT, none of the modes are active. In the low temperature, low symmetry phase, all four modes are active. There exists a region of temperature in between these phases in which the polar modes are active, and the silent mode is not. Possibly there exists an intermediate phase of different symmetry between the two established phases that could explain this.

At least in KLT, the $\mathrm{TO}_{3}$ mode appears concurrently with a ferroelectric phase transition, with behaviour independent of polar ferroelectric modes. It is a little lacking in rigour to assert that the same must be true of the $\mathrm{TO}_{3}$ mode in STO. In the cases I have studied in STO, there have been a few cases where $\mathrm{TO}_{3}$ appears. In some of those cases, ferroelectricity was measured directly through permittivity measurements or hysteresis loops. In the polycrystalline thin film case, ferroelectricity is very unlikely, although we cannot rule it out because we do not have electrical measurements on the same sample as the one $\mathrm{TO}_{3}$ was detected in in the Raman. It seems likely on this basis, that there are some non-ferroelectric phases of STO which can still show $\mathrm{TO}_{3}$. However, the fact that there exist lowsymmetry, non-ferroelectric phases in which $\mathrm{TO}_{3}$ is silent is still helpful - it must be used in combination with other spectral features.

\subsubsection{General themes in STO systems}

Polar micro-regions and the percolative phase transition

A major theme in the literature on STO is that of polar microregions (also variously referred to as ferroelectric micro-regions, and local symmetry breaking regions). Whatever the nomenclature, the term refers to an inhomogeneity within 
STO where there is a coexistence of phases in some temperature ranges inside the material[39]. These regions are attributed to various different sources, such as grain boundaries, interstitial cation defects, or ${ }^{18} \mathrm{O}$ isotopes and the mechanism described is always largely the same. These defects in the system can cause electric fields. This can be an intrinsic effect, such as with interstitial ions, or it can be achieved by causing one or more unit cells to enter a structure that has a dipole moment, such as with ${ }^{18} \mathrm{O}$ substitution. The electric fields extend a short distance into the material, and induce a lower symmetry phase in the unit cells around the defects. In these regions, the vibrational modes that are Raman silent in the cubic phase of STO are no longer silent, and are seen in the Raman spectrum. The ability of electric fields to induce lower symmetry phases in STO is well established[29][56]. As the temperature is lowered, the polar regions grow. In some samples, they grow large enough to start merging and percolating, eventually forming a cohesive phase of lower symmetry throughout the whole crystal. This growth and percolation of polar microregions is thought to be the main mechanism for the ferroelectric phase transition in STO systems, a very different mechanism to a first-order displacive phase transition that is coherent throughout the material. Even in cases where the phase transition occurs over a very small temperature range, there is strong evidence for this mechanism as a mediator of the phase transition[38].

The cited paper further makes a distinction between local ferroelectric regions, and local symmetry breaking regions that are not ferroelectric. They deduce that both types of region are present in their samples, which are prepared with concentrations of ${ }^{18} \mathrm{O}$ near to, but under the critical concentration for ferroelectricity. It is important to be aware of this compexity in the ferroelectric phase transition of STO when interpreting the Raman spectra.

Ferroelectric soft mode behaviour

Another major theme in the literature for STO is the behaviour of the ferroelectric soft mode $\left(\mathrm{E}_{U}\right.$ symmetry in the tetragonal phase, often called $\mathrm{TO}_{1}$ in IR and Raman studies). This mode is more easily seen in IR measurements, so I will be drawing on IR as well as Raman measurements in discussing this mode. As mentioned in section 2.3, the ferroelectric soft mode in pure STO softens towards 
zero, appearing to go towards a phase transition by the freezing of the mode at zero frequency. However, at roughly the same temperature that deviation from CurieWeiss behaviour in the permittivity is detected, the mode slows in its softening, never falling to zero and reaching only about $10 \mathrm{~cm}^{-1}$.

Various factors can act to either slow down the softening further, or to increase it such that it does reach near zero levels, and subsequently start hardening again. In both of the most clear examples of ferroelectric STO, Ca-substituted[43] and ${ }^{18} \mathrm{O}$ substituted single crystals[38], softening to near zero and then hardening is observed in this mode, and it is a very clear indication of a phase transition. Conversely, in many cases where permittivity is considerably weaker than in pure STO, a striking lack of hardening is observed[57]. In studies of fine-grained ceramics of $\mathrm{STO}[22]$, the dielectric permittivity was found to be on the order of one tenth the strength of single crystals, and the ferroelectric soft mode softens no further than $60 \mathrm{~cm}^{-1}$, about six times the frequency of the single crystal case. The presence of free conductors in the material also has this effect[58]. Electric fields have also been shown to harden the soft mode in STO thin films[59][30] and in ${ }^{18} \mathrm{O}$ substituted $\mathrm{STO}[60]$. In the latter case, the E-field also caused a splitting of the soft mode. There have also been reported cases where the ferroelectric soft mode softens to a value greatly above zero frequency $\left(40 \mathrm{~cm}^{-1}\right)$, then reverses direction and starts hardening again from that raised frequency[44]. This sample was shown not to be ferroelectric by permittivity measurements, and shows a similar weakness of permittivity to the other 'weak ferroelectric mode softening' cases.

So in general, a ferroelectric soft mode which displays strong softening and subsequent hardening is a good sign of ferroelectricity that we may see in the Raman. Conversely, a soft mode at frequencies greatly above zero frequency suggests no ferroelectricity, and a weakened dielectric response. These may not be hard and fast rules, but they are what is commonly seen in the literature.

Muddying the waters of this analysis somewhat is the common existence of polar microregions. In each of the different types of region, there is potentially a different soft mode behaviour. One must keep this in mind when interpreting the Raman spectra. This is explicitly used in the analysis of[38] to explain the overall Raman spectrum, as a sum of contributions from the ferroelectric regions and the non-ferroelectric regions. 


\subsubsection{Summary of Raman signatures of ferroelectricity in STO}

A summary of the features of the STO Raman spectrum that signal, or may signal ferroelectricity is useful before examining my results. For clarity, the features that are assigned here as being associated with ferroelectricity are those that are associated with a peak in dielectric permittivity.

Strong indicators of a ferroelectric phase in the Raman:

- Ferroelectric soft mode $\left(\mathrm{E}_{u}\right.$ symmetry in AFD tetragonal phase; $75 \mathrm{~cm}^{-1}$ at $300 \mathrm{~K}$, drops towards $0 \mathrm{~cm}^{-1}$ as temperature tends towards $0 \mathrm{~K}$ )

- Temperature dependent softening above $\mathrm{T}_{C}$, eventually reaching nearzero frequency

- Hardening, and huge increase in intensity below $\mathrm{T}_{C}$

- Splitting into three components below $\mathrm{T}_{C}$

- AFD lower-frequency soft mode component $\left(\mathrm{E}_{g}\right.$ symmetry in AFD tetragonal phase, $20 \mathrm{~cm}^{-1}$ at $15 \mathrm{~K}$ )

- Splitting into two components below $\mathrm{T}_{C}$

NB - although a strongly softening ferroelectric soft mode is a very good indication of a ferroelectric phase transition, a hardened ferroelectric soft mode is conversely a strong indicator of a lack of a ferroelectric phase.

Inconclusive indicators of a ferroelectric phase:

- Polar hard modes - $\mathrm{TO}_{2}\left(175 \mathrm{~cm}^{-1}\right), \mathrm{TO}_{4}\left(450 \mathrm{~cm}^{-1}\right), \mathrm{LO}_{4}\left(800 \mathrm{~cm}^{-1}\right)$

- Rapid rise in polar hard mode intensity - only rise over a small temperature range is a good indicator of ferroelectricity. A rise occurring over larger temperature ranges is an indicator of nucleation and growth of local symmetry breaking regions, but not necessarily of a macroscopic phase transition. 
- Non-polar hard mode $\mathrm{TO}_{3}\left(267 \mathrm{~cm}^{-1}\right)$

- This mode, silent in the cubic and tetragonal phases of STO is active in the orthorhombic, ferroelectric phase. There is evidence of nonferroelectric phases of STO in which this mode is silent where other first order modes are active. However, there also exist non-ferroelectric phases of STO in which this mode is active. This mode is overall less prone to be activated by polar micro-regions and other precursors to ferroelectricity than the polar hard modes.

None of these hard modes are as good an indicator of a ferroelectric phase as either of the soft modes. However, their higher frequency makes them easier to observe. In both cases, temperature dependence of the modes can tell us far greater amounts about the symmetry and phase transitions than a spectrum at low- $\mathrm{T}$ alone. Obviously, the temperature range over which the mode intensity increases is a continuous variable, and although there is a clear phase transition at one end, and confirmed cases of non-ferroelectric STO at the other, the forms taken in between are not well described by either 'displacive phase transition' or 'paraelectric.' It is difficult to draw a line separating the two, so this factor should be considered together with other, more reliable features for best results. There is also a continuous scale of ferroelectric properties in STO - seen most clearly in the substantial body of literature on Ca-substituted STO. There is a threshold at around 1\% Ca-substitution activating a ferroelectric phase transition. increased Ca-substitution beyond this threshold is associated with a broader temperature range of polar mode intensity increase, and simultaneously with weaker ferroelectric properties.

Using the method of temperature dependent ultraviolet Raman spectroscopy, I have measured STO thin films epitaxially grown on various lattice-mismatched substrates, and analyzed the results using the above framework. 


\section{Chapter 3}

\section{Experimental methods and materials}

In this chapter, the experimental methods and materials are detailed. First, I list all of the samples used in this thesis, including details on their preparation. Next, the experimental techniques and apparatus are described. Finally, I discuss the choice of Raman spectrometers and temperature control systems available to me.

\subsection{Sample information}

\subsubsection{BNT-BT system}

The samples used in this section of the thesis were ceramic pellets of $\left(\mathrm{Na}_{0.5} \mathrm{Bi}_{0.5}\right)_{1-\mathrm{x}} \mathrm{Ba}_{\mathrm{x}} \mathrm{TiO}_{3}$, with $x=0,0.025,0.05,0.06,0.07$, and 0.09. They were fabricated by standard solid-state synthesis using $\mathrm{Ba}$ and $\mathrm{Na}$ carbonates and $\mathrm{Ti}$ and $\mathrm{Bi}$ oxides. For the poling study, the ceramics were poled at $333 \mathrm{~K}$ in a field of $60 \mathrm{kV} / \mathrm{cm}$ for 10 minutes. The synthesis was carried out by Naama Klein at the Swiss Federal Institute of Technology (EPFL). 


\subsubsection{PT nanowires system}

The samples used in this section of the thesis were nanowires of PT. The preparation of these wires is detailed in the following papers[3][61], and is repeated here for completeness: First, nanowires of $\mathrm{PTiO}_{3}$ were prepared in the 'PX' phase using hydrothermal methods. The sample was prepared with a hydrothermal method similar to what is reported by Cheng et al.[61]. "For PX-phase PT, 4 mmol Ti $\left(\mathrm{OC}_{4} \mathrm{H}_{9}\right)_{4}$ was first dissolved in $8 \mathrm{~mL}$ of ethanol and the resulting transparent solution was hydrolyzed in $8 \mathrm{~mL}$ of deionized $\mathrm{H}_{2} \mathrm{O}$. Subsequently, $20 \mathrm{mmol}$ of $\mathrm{KOH}, 5.2 \mathrm{mmol}$ of $\mathrm{Pb}\left(\mathrm{CH}_{3} \mathrm{COO}\right)_{2} \cdot 3 \mathrm{H}_{2} \mathrm{O}$, and $0.050 \mathrm{~g}$ of polyvinyl alcohol (PVA) were added and mixed under stirring. The volume of the final feedstock was adjusted to $40 \mathrm{~mL}$ with deionized $\mathrm{H}_{2} \mathrm{O}$. The ratio of $\mathrm{Pb} / \mathrm{Ti}$ in the starting material was kept to 1.3 to ensure a complete reaction of $\mathrm{TiO}_{2} \cdot \mathrm{n}\left(\mathrm{H}_{2} \mathrm{O}\right)$ gel. The $40 \mathrm{~mL}$ feedstock was transferred to a $50 \mathrm{~mL}$ Teflon-lined autoclave and held at $200^{\circ} \mathrm{C}$ for $3.5 \mathrm{~h}$. The products were washed with deionized $\mathrm{H}_{2} \mathrm{O}$ until a neutral $\mathrm{pH}$ is achieved and then washed in $10 \mathrm{wt} \% \mathrm{CH}_{3} \mathrm{COOH}$ solution to remove the remnant $\mathrm{PbO}$. The final pure PX-phase sample was obtained by another cycle of washing with deionized water and dried at $60^{\circ} \mathrm{C}$ in air."

In order to transform the PX-phase wires into perovskite PT phase, the PX nanowires were annealed in air at about $540^{\circ} \mathrm{C}$. The resulting wires were between 20 and $500 \mathrm{~nm}$ in thickness. The above material synthesis was performed by Jin Wang at the Swiss Federal Institute of Technology (EPFL).

For Raman measurements and microscope and scanning electron microscope (SEM) imaging, we wanted to look at single nanowires. Therefore, a small mass of PT wires were diluted into isopropyl alcohol. The solution was sonicated to separate the wires, and then the dilute solution was evaporated onto sapphire substrates. This yielded disperse nanowires such that a single wire could be put into the Raman laser spot. Two separate samples of wires dispersed onto substrates are used in this study. Sample 1 had a higher concentration of nanowires dispersed onto the surface. Sample 2 was was more dilute. 


\subsubsection{STO thin films}

The following samples of strained thin STO films grown by pulsed laser deposition (PLD) were used in this section of the thesis:

- STO016: $50 \mathrm{~nm}$ of STO grown by PLD on a (001) STO substrate

- STO267: $100 \mathrm{~nm}$ of STO grown by PLD on a $\mathrm{LaALO}_{3}$ (001) substrate

- STO263: $30 \mathrm{~nm}$ of STO grown by PLD on a $\mathrm{LaALO}_{3}$ (001) substrate

- STO264: $50 \mathrm{~nm}$ of STO grown by PLD on a NdGaO3 (110) substrate

- STO004: $60 \mathrm{~nm}$ of STO grown by PLD on a $\left(\mathrm{LaAlO}_{3}\right)_{0.29} \times\left(\mathrm{SrAl}_{0.5} \mathrm{Ta}_{0.5} \mathrm{O}_{3}\right)_{0.71}$ (LSAT) (001) substrate

- STO005: $140 \mathrm{~nm}$ of STO grown by PLD on an LSAT (001) substrate

- STO006: 10 nnm of STO grown by PLD on an LSAT (001) substrate

The samples were synthesised by using pulsed-laser deposition to create epitaxial growth onto the substrates listed. The substrates have lattice mismatches with STO and so the epitaxial films are under strain, which is predicted to cause ferroelectric phase transitions in STO[1]. On some of the samples, in-plane XRD measurements have been made to find the actual strain in the samples. These measurements are not my contribution. XRD measurements for the other strained films are not available at time of writing, but are planned. These samples together with their lattice mismatch and measured strain (where available) are presented in tabular form below.

\begin{tabular}{lllll}
\hline Sample name & Substrate & Lattice mismatch & Thickness & Measured strain \\
\hline STO016 & STO & $0 \%$ & $50 \mathrm{~nm}$ & $\mathrm{n} / \mathrm{a}$ \\
\hline STO263 & LAO & $-3.06 \%$ & $30 \mathrm{~nm}$ & $1.1 \%$ compressive \\
\hline STO267 & LAO & $-3.06 \%$ & $100 \mathrm{~nm}$ & $0.7 \%$ compressive \\
\hline STO264 & NGO & $-1.0 \%,-1.3 \%$ & $50 \mathrm{~nm}$ & $1.3 \%$ compressive \\
\hline STO004 & LSAT & $-0.95 \%$ & $60 \mathrm{~nm}$ & not available \\
\hline STO005 & LSAT & $-0.95 \%$ & $140 \mathrm{~nm}$ & not available \\
\hline STO006 & LSAT & $-0.95 \%$ & $105 \mathrm{~nm}$ & not available \\
\hline \hline
\end{tabular}


The synthesis of these samples was carried out by Tomoaki Yamada at the Tokyo Institute of Technology and Nagoya University, except for those samples grown on LSAT, which were synthesised by the author at Nagoya University. In Chapter Six of this thesis, a strain is estimated for those films that we do not have a measured value for.

\subsection{Experimental methods}

\subsubsection{BNT-BT}

The samples were placed into a Linkam THMS600 liquid nitrogen cooled cryostat, and mounted on the microscope stage of a Jobin-Yvon T64000 Raman spectrometer operated in triple subtractive mode. Spectra were detected in quasi-backscattering geometry (distorted from true backscattering geometry by the microscope objective). The detection unit was a liquid nitrogen cooled charge-coupled device. The $514.5 \mathrm{~nm}$ line of a Ar-Kr gas laser was used for excitation with power kept at $100 \mu \mathrm{W}$ incident on the sample, and a monochromator was used to exclude plasma lines. A 0.9 numerical aperture microscope objective was used for imaging and collection. 1800 line/mm diffraction gratings were used in the premonochromator of the T64000 as well as the third stage spectrometer. This experimental setup gave a spot size of diameter $3 \mu \mathrm{m}$ and resolution of $5 \mathrm{~cm}^{-1}$. As the samples were ceramic, the spectra are of mixed polarisation.

The samples were first cycled down to $73 \mathrm{~K}$, and then up above $500 \mathrm{~K}$, above the transition temperature for depoled samples, and then back to ambient temperatures again. This was done to preserve the poling at low temperatures, and to observe any hysteresis resulting from the cycling above the phase transition temperature.

\subsubsection{PT nanowires}

Sample 1 (the more concentrated sample of PT nanowires dispersed on sapphire) was loaded into the Linkam THMS600 cryostat and the T64000 Raman spectrom- 
eter. The same basic configuration was used as described for BNT-BT, with the following exceptions: Additional microscope objectives, of numerical aperture 0.50 and 0.25 were used for imaging, though for the collection of the spectra the 0.9 numerical aperture objective was still used resulting in a spot of diameter $3 \mu \mathrm{m}$. The laser power was increased to as much as $1 \mathrm{~mW}$ incident on the spot in order to increase signal from the smaller samples (note though that this was the power in the whole spot and that especially in the case of the smallest wires, the spot would be considerably larger than the wires measured). Because of the higher power, we checked for laser heating effects by seeing if there was any difference in the transition temperature with low and high laser powers. For this, the larger wires with the highest signal were used and no effect of laser heating was measured. The entrance slits were closed further than for the BNT-BT measurements resulting in a higher resolution of $3 \mathrm{~cm}^{-1}$. The crystal orientation of the nanowires was not known at the time of study, and different samples had different in-plane orientations. The excitation light was polarised in-plane (with distortion caused by focusing of the microscope objective), and light was collected in all polarisations to form the spectra.

Bundles of wires were measured with the spectrometer as the easiest wires to identify and excite with the laser spot. The sample was heated up to $600{ }^{\circ} \mathrm{C}$ and down to liquid nitrogen temperature, in an initial investigative study to which individual and smaller wires could be compared. Using the optical camera alone, small wires were unable to be located. So the next step was to use electron microscopy to find appropriate wires.

Sample 1 was imaged in a scanning electron microscope, with the goal of locating and identifying nanowires of different diameters. Ordinarily for making SEM images on weakly conducting samples, one would coat the sample with a few nm of gold or platinum to conduct away charge accumulated by the imaging beam. Such a coating is commonly made before SEM imaging to prevent buildup of charge. Charge building up on the sample is a consequence of firing electrons at it. It ruins image contrast, and happens over time, proportional to the electron imaging flux, and inversely proportional to the conductivity of the sample. Giving a very thin conductive overlayer to a sample helps transport away the charge that builds up, and allows long integration images which would otherwise be impossible on low conductivity samples. Our samples are low conductivity, however we wanted 
not to coat the samples at this stage of the experiment because we wanted not to alter the Raman signal. This decision did impact on the resolution and image quality we were able to achieve. Charging of the substrate meant that we could not get high resolution images of nanowires with the smallest diameters, $<100 \mathrm{~nm}$. Rough images of these wires were taken in this initial run, including maps. These images were good enough to locate the wires in the optical camera, by using larger wires and structures as landmarks. However, they do not give an accurate image or measure of the diameter of the smallest of the wires.

Sample 1 was then loaded into the Linkam THMS600 cryostat and the T64000 Raman spectrometer. Wires identified and measured for size in the initial SEM images were located using the series of microscope objectives of different magnifications. The temperature controller was then used to make temperature dependent Raman measurements of these samples. The signal from the smaller wires was very weak, which made long collection times ( $\sim 10$ minutes) necessary to see a signal. However, due to the spatial drift of the microscope stage and the small areas being focused on, the laser spot would drift off the smallest wires in this time period. Additional mechanical clamping of the cryostat to the microscope stage alleviated the problem somewhat, however on some samples it was necessary to take many one minute collections instead of a single longer one, and add the results together in post.

After measurement of sample 1 failed to yield any nanowires for study below $100 \mathrm{~nm}$ in diameter, the more dilute sample 2 was examined. First SEM imaging was performed on it in the same way described above, and this imaging was able to find a large number of sub $100 \mathrm{~nm}$ wires.

Sample 2 was subjected to temperature dependent Raman spectroscopy, from room temperature up to $500^{\circ} \mathrm{C}$ where a step change was observed in the spectra, and then were cooled back to room temperature.

Finally, after Raman measurements of sample 2 was complete, another round of SEM imaging was done on this sample, this time with gold coating in order to increase the conductivity of the surface and allow better imaging of the smallest wires. 


\subsubsection{Strained STO thin films}

UV Raman spectroscopy was the tool used to measure these strained STO thin films due to its surface selectivity. Making these measurements necessitated the creation of new optical paths for excitation and imaging around the T64000 spectrometer.

The as-prepared films were measured using a Jobin-Yvon T64000 Raman triple spectrometer, run in triple subtractive mode in two different configurations. In the first configuration, samples were mounted in a closed-cycle helium cryostat, which could measure down to $8 \mathrm{~K}$ in pressure of approximately $1 \times 10^{-5}$ Torr, and were measured. The excitation source was the $325 \mathrm{~nm}$ line of a He-Ne gas laser, and a solid plasma line filter was used to exclude plasma lines. The detection element was a liquid nitrogen cooled charge coupled device, and the dispersive elements used were 2400 line/mm diffraction gratings, blazed in the UV. The sample-window distance in the cryostat was too great to use a microscope objective for imaging and collection, so an alternative system of UV lenses needed to be constructed. The laser beam was focused to a spot on the sample using a fused silica lens with a focal length of $150 \mathrm{~mm}$, numerical aperture 0.084. To image the spot on the spectrometer's focal plane, a fused silica lens with focal length $90 \mathrm{~mm}$ and numerical aperture 0.34 was used. This collection lens was mounted on linear stages to grant fine three-axis translational control over its position. Raman spectra were collected at approximately 45 degrees to the excitation, and unlike in microscope objectives, this focusing lens did not significantly alter the angle of incident light. A schematic of this imaging system is shown in Fig. 3.1. This optical setup resulted in a spot size on the sample of $55 \mu \mathrm{m}$, and a magnification of 9.2 between the sample and the spectrometer imaging plane - therefore the spot size at the spectrometer entrance was roughly $500 \mu \mathrm{m}$. The total power within this spot was approximately $3 \mathrm{~mW}$. The larger spot size restricted our ability to improve resolution without losing signal, so the resolution in most of the spectra of STO thin films is $5 \mathrm{~cm}^{-1}$ - somewhat less well resolved than in BNT-BT. For some spectra, the resolution lower than this figure at about $10 \mathrm{~cm}^{-1}$. Where this is the case, it is noted in the caption to the spectra. The light incident on the sample is of mixed polarisation relative to the sample crystal axes, and the collected light is likewise of mixed polarisation in these experiments. 


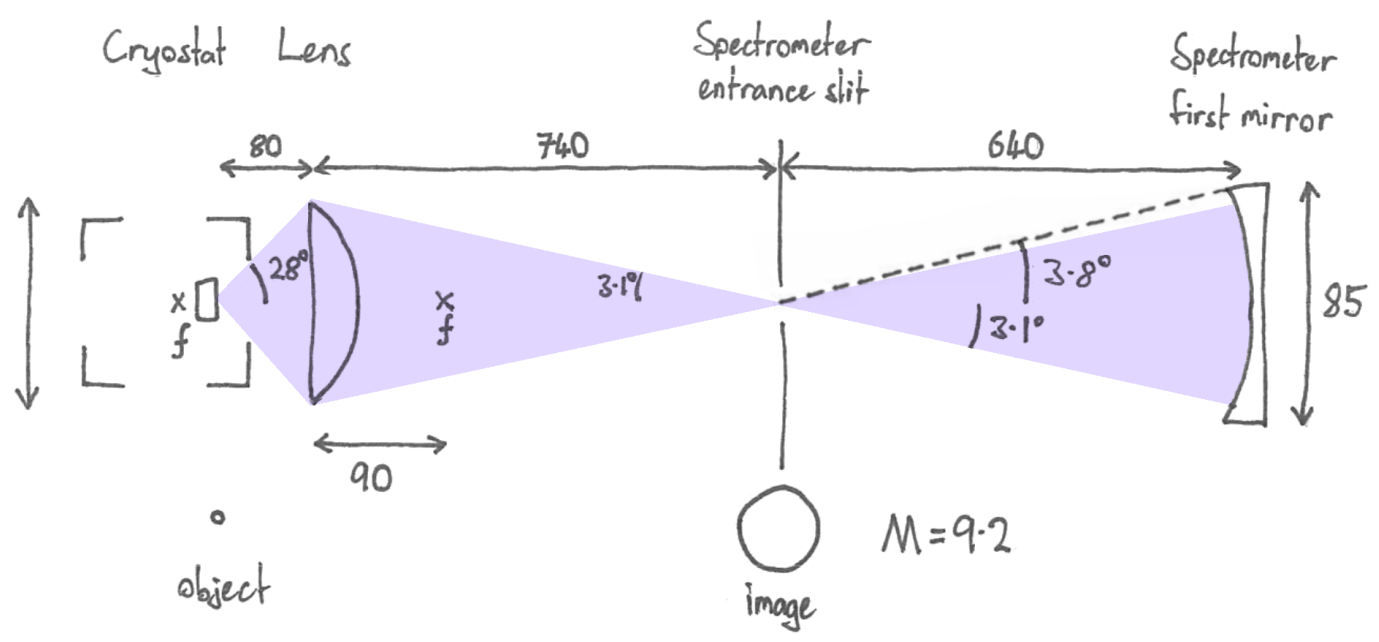

Figure 3.1: Schematic of UV optical path between sample and spectrometer. This figure is not drawn to scale, but it shows the light cone collected, the magnification caused by the positioning and numerical aperture of the chosen UV lens, and the filling of the spectrometer first mirror. Distances shown are measurements in $\mathrm{mm}$.

In choosing the optical components for the imaging in this configuration, we faced a trade off between throughput and maximum resolution. The signal from these thin films is very weak, and making temperature dependent measurements is a slow process. For such long timeframe measurements, sources of error other than random counting noise start to have greater effects - unequal response of CCD pixels, instability in the laser power and temperature control, interference from cosmic rays to name a few. It is more optimal to collect with a higher throughput and shorter collection time to reduce sources of error, so we wanted to collect the largest possible solid angle of scattered light from the sample. However, as the solid angle of collection grows larger, the mismatch between the effective numerical aperture of the spectrometer, and that of the lens system also grows. The result of this is a magnification of the laser spot image upon the slits of the spectrometer. The magnification of the spot image restricts our ability to improve resolution of the spectra by closing slits. The solid angle collected by the chosen system was a 28 degree cone, and this resulted in a magnification factor of 9.2. This produced an image of the laser spot that was approximately $500 \mu \mathrm{m}$ in diameter. Using this light cone resulted in a slight under-filling of the first spectrometer mirror, so none of the collected light was lost in the dispersion. 
The second configuration had the samples mounted in the Linkam THMS600 stage. With this stage, it was possible to use microscope objectives to collect light from the sample - but not to reach temperatures below $77 \mathrm{~K}$. The configuration was the same as the other UV T64000 lens configuration reported above with the following exceptions: A UV-transparent microscope objective of numerical aperture 0.32 was used for both imaging and collection. The resolution and laser power in this configuration were the similar to the helium cryostat configuration, but the spot size was approximately $35 \mu \mathrm{m}$. In this configuration, both incident light and collected light were of mixed polarisation relative to the crystal axes.

In addition to Raman spectroscopy, lab-XRD was performed on the samples grown on LSAT substrates. a Bruker D8 Discover XRD system was used to perform the measurement, with excitation from the copper $\mathrm{K} \alpha$ line. In these experiments, the out-of-plane lattice constants of the samples were measured.

\subsection{Experimental apparatus}

The vast bulk of the work in this thesis consisted of taking temperature dependent Raman spectra of various samples, so a more in depth investigation of the temperature control and Raman spectrometer systems that were used is warranted.

\subsubsection{Discussion of spectrometer systems}

The main difference between the T64000 and the Labram spectrometers is in the resolution that each system is able to achieve. The T64000 is able to achieve much higher resolutions than the Labram, but it does so at the cost of higher throughput. The Labram has significantly higher counts/sec in normal conditions.

There are four main elements of a spectrometer that influence the resolution. In no particular order, they are dispersion, focal length, detection resolution, and slits. See Figs. 3.2 and 3.3. Dispersion is the ability of the spectrometer to split light into different frequency components, it is a measure of the angular spread. Higher dispersion will push light rays further apart in space, resulting in higher 
resolution. The dispersive elements in both the Labram and T64000 are the same. Diffraction gratings are used, and both systems have gratings up to 2400 lines per mm available. Focal length is another factor in resolution of spectrometers. The greater the distance between the dispersive element and the detector, the further apart light of different frequencies will be in a direction transverse to the beam path. Therefore, at the CCD where the signal is detected, each pixel will collect a smaller range of frequency, and the resolution will be consequently higher. Put another way, the same frequency range would be covered by more pixels in the longer focal length case. In this category, the Labram has a slight resolution advantage. Its dispersion length is $800 \mathrm{~mm}$ compared to $640 \mathrm{~mm}$ for the T64000. (Note though that the T64000 can be run in additive mode, in which each of the three sets of gratings is used to disperse, giving an effective focal length of $1920 \mathrm{~mm}$. This mode was not utilised in the current study, as it has far inferior capacity to detect low frequency signals, and it suffers from an overall drop in intensity when the measurements we would like to make are already weak).

The detector resolution also influences the spectral resolution of the spectrometer. The smaller the detection elements spatially, the higher a spectral resolution is achieved. Both the Labram and the T64000 use charge coupled devices for detection, and so the size of the pixels determines the resolution. The CCDs used by each system are the same in terms of their pixel size.

Those three factors determine the resolution assuming a single light beam entering the spectrometer on-axis. However, sample beams (and sample images) have non-zero spatial extent. Light entering the spectrometer at a point that is not the optical axis will be dispersed in the same way - but from a different point of origin on the dispersion element. This blurs out the spectrum picked up by the detector, leading to decreased resolution. In order to reduce the broadening from this factor, it is necessary to place slits on the optical axis, and close them down as much as possible. The T64000 has considerably better tools for reducing this broadening than the Labram. Whereas the labram has only a single pinhole for this purpose, the T64000 has a series of three slits. Each of the slits has good tools for finely controlling the position of the beam within the slit, making the alignment process considerably easier. The fact that the slits are single dimension restriction, rather than the pinhole which is $2 \mathrm{D}$ restriction gives the T64000 much better throughput at small slit widths as well - closing a pinhole decreases the light 

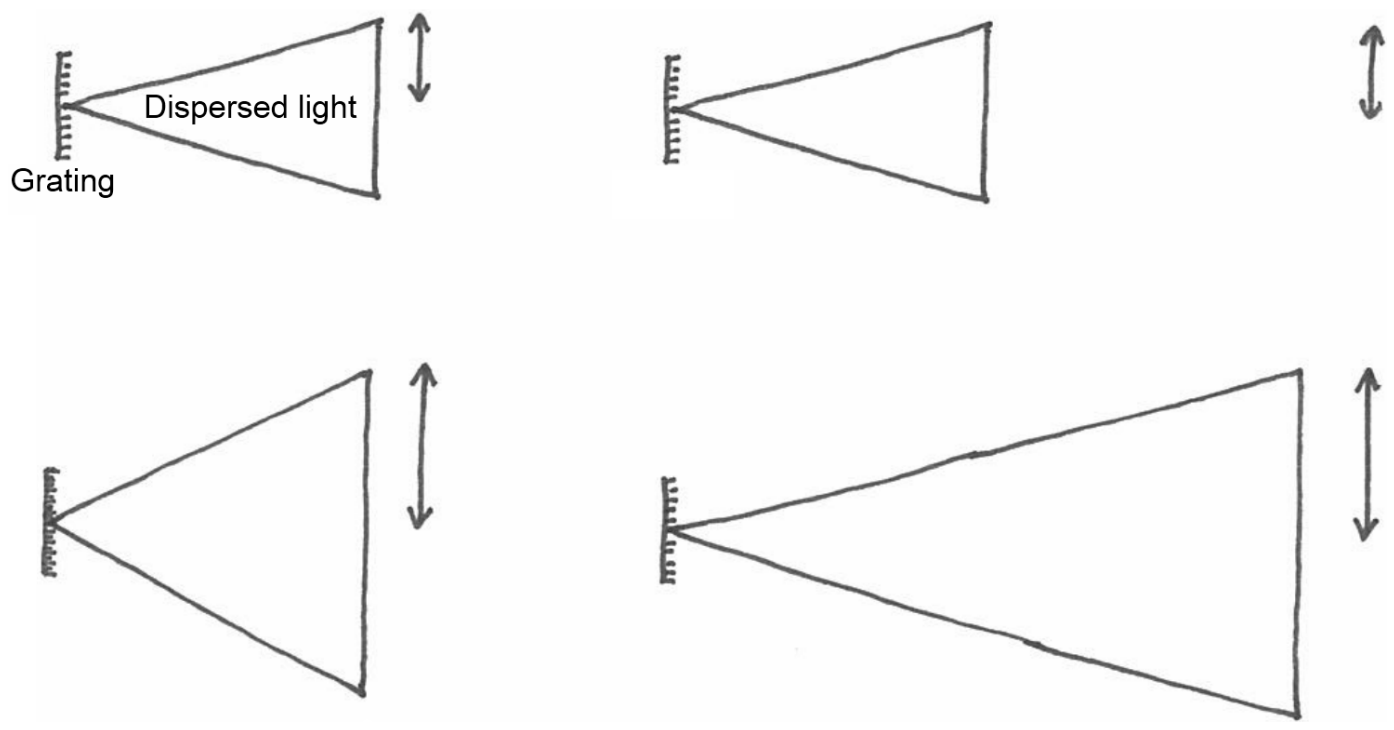

Figure 3.2: Sources of line broadening in Raman spectroscopy. Left: Dispersion. Higher dispersion $=$ greater resolution. Right: focal length. Greater focal length $=$ greater resolution .

cross section as $1 / d^{2}$ whereas closing a $1 \mathrm{D}$ slit only reduces it by $1 / d^{1}$. When run in triple subtractive mode, the T64000 uses two of its three slits to achieve higher spectral resolution (the first and third slits are used for this purpose). The second slit is used to reduce the spectral window, and reject the Rayleigh scattered line.

As the slit-width induced broadening is the dominant factor in the overall instrumental broadening for the experiments we want to perform, the advantage in high resolution measurement goes to the T64000. The system is easier to align for high resolution measurements, and has a higher throughput during them due to the superior number, fine control and geometry of the slits.

The difference in the method of Rayleigh light rejections is the second major difference between the two spectrometers. The Labram system uses a notch filter to achieve rejection of the Rayleigh scattered light. Notch filters are sophisticated band stop filters. They operate, in general, by using a periodic fluctuation inside a material at the exact wavelength of the light to be rejected inside the material. The effect of this is that the material becomes highly reflective to light of that wavelength, without affecting other wavelengths overly much, a product of constructive interference. The best notch filters for Raman spectroscopy are made by recording an interference pattern between two lasers in a gel structure. This gives 

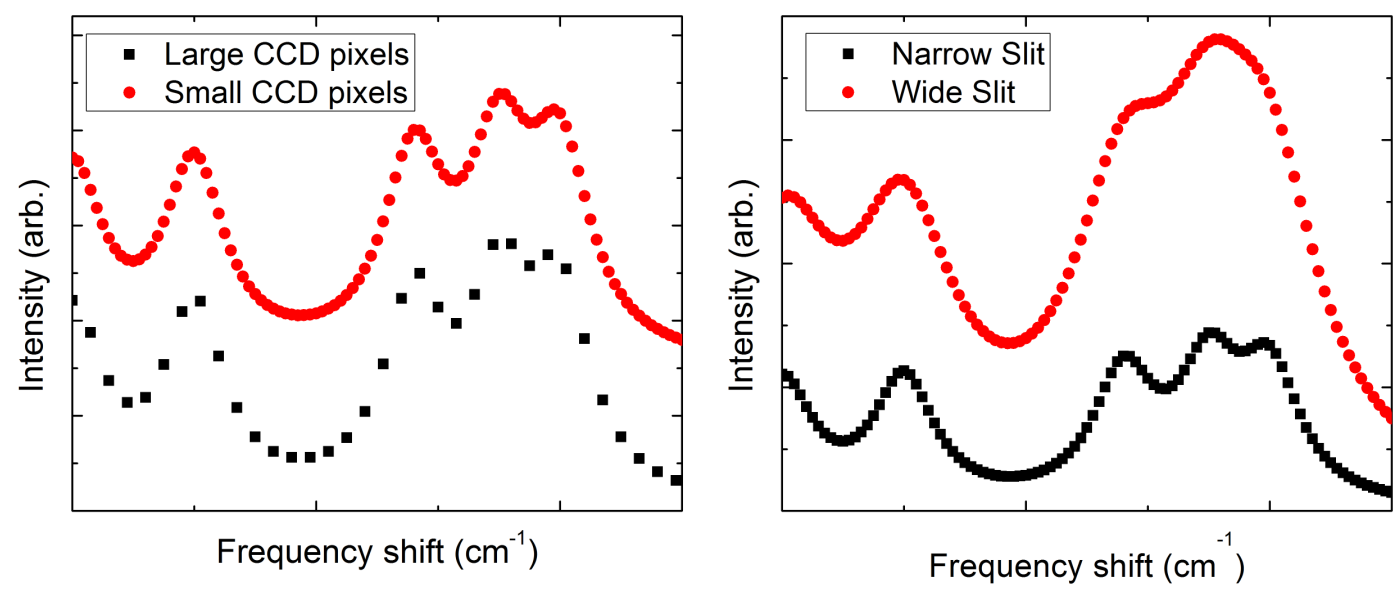

Figure 3.3: More sources of broadening in Raman spectroscopy. The fabricated spectra have been vertically offset for ease of viewing. Left: Detection pixel size. Smaller pixels $=$ greater resolution. Right:Slit width. Smaller slit width $=$ greater resolution. BUT - also smaller throughput.

a high level of coherence to the pattern, which results in a very narrow band stop interval, and the sinusoidal pattern is superior to a square-wave pattern as might be created by thin solid layers due to the lack of reflections.

As discussed above, the T64000 (when run in triple subtractive mode) uses an intermediate slit to reject Rayleigh scattered light. The collected light is gathered, then dispersed in the pre-monochromator. The light is then windowed by the slit, blocking the Rayleigh scattered light. After this filtering, the light is then refocused onto the third stage spectrometer grating and dispersed onto the CCD. This method allows for very fine adjustment of the spectral window, and also has a much sharper spectral cut-off than that provided by a notch filter. When working close to the laser line and attempting to resolve peaks in the low wavenumber region $\left(<100 \mathrm{~cm}^{-1}\right)$ the $\mathrm{T} 64000$ is the best of the two systems to use.

\subsubsection{Discussion of temperature control systems}

Two temperature control systems were used in the Raman experiments of this thesis. The first is a Linkam THMS 600 nitrogen cooled cryostat, and the second is a closed-cycle helium cryostat. The Linkam stage's main advantage is its short window-to-sample distance, close enough to allow a long working distance micro- 
scope objective to focus and image the laser spot. Using a microscope objective has numerous advantages, including higher throughput, smaller spot size, short depth of field and confocal ability, and microscope (white light) imaging to pick out small features. However, there are one or two disadvantages to using microscopes as well. The main one is that polarisation information of the incoming light is easily lost due to the high angles the objective turns the beam at. The result is mixed polarisation excitation, which is less useful for making polarisation dependent measurements. The degree of mixing is proportional to the numerical aperture of the objective of course, so using a low N. A. objective can usually mitigate this problem to a sufficient degree for most experiments. The helium cryostat system requires a macroscopic lens to create a focused spot on the sample. In theory, a lens with focal length a minimum of $30 \mathrm{~mm}$ could be used with this cryostat, but in practice a $150 \mathrm{~mm}$ lens is used. This allowed more space between the focusing and collecting lenses so that adjustments could be made. The longer focal length lenses have a greater depth of field, and a smaller numerical aperture, so the spot they can create on the sample is larger and less focused than that of the microscope, leading to lower throughput. While the separation of focusing and collection lenses in this set up does allow for measurements to be made in a number of configurations besides back-scattering, the need to align two lenses instead of one is a hassle. It is not always the case that a small spot size is an advantage, however. When measuring microstructured samples, or anything else that may be expected to show variation on a micron length scale, it is indispensable. However, when measuring samples that are consistent on a micron length scale, it makes the measurements more sensitive to precise positioning of the sample. When measuring the thin STO films, the short depth of field given by the microscopes gave a variation in the signal as the sample surface shifted up and down due to thermal expansion and contraction. This introduced a new source of experimental error into the measurements. However, using the long focal length lenses in the macroscopic configuration, the depth of field was much larger than the sample, and the variation in sample height was not sufficient to produce an altered signal. As a consequence, the macro measurements are more consistent than the micro measurements in that case.

The main advantage of the helium cryostat is of course its low temperature range. Refrigeration in the helium cryostat is achieved via closed-cycle expansion 
and contraction of helium gas through the Gifford-McMahon cycle. The sample is mounted on a copper block using vacuum grease for good heat conduction inside a cold finger. The refrigerator is always on at constant power, and variable resistive heating is used to control the temperature, the heating is controlled automatically by a microcontroller. This allows measurements in practice of temperatures as low as $8 \mathrm{~K}$. This cryostat is not designed to cope with high levels of heat extraction though, so it cannot be operated above room temperature. Temperature control in the Linkam cryostat is achieved through flow of liquid nitrogen through the metal hearth on which the sample rests, and resistive heating in the same hearth. Both the resistive heating and the nitrogen flow rate are variable, and the function is similar to a coarse-fine control system, with coarse adjustments made using the nitrogen flow level, and fine adjustments made by varying the resistive heating. The nitrogen can be controlled manually, and the resistive heating is automatic, controlled by computer program. The nitrogen flow is not closed cycle, and nitrogen is consumed in the process. Because of this, continuous measurements are limited by the capacity of the nitrogen dewar, giving a limit of as low as one or two hours at maximum flow rate (i.e., minimum temperature). This time limit has been problematic on occasion in this work. The Linkam has a temperature range of between $-195^{\circ} \mathrm{C}$ to $600{ }^{\circ} \mathrm{C}$. At high temperatures, water cooling flow is required to avoid overheating of the outside of the cryostat, and melting of electronic components. We also noticed that operating near the high temperature limit in the Linkam resulted in cracking of the cryostat window. Whether this was caused by high temperatures alone, or by the change in temperature during cooling from high temperatures we are not certain. One final note about the temperature range of the two systems is that the Linkam is considerably more agile through most of its temperature range. Changes in temperature can be made rapidly, at $40 \mathrm{~K}$ per minute without introducing faults or breakages and potentially higher, though this was not tested. The helium cryostat is very slow on cooling from room temperature to below $50 \mathrm{~K}$. That process takes one or two hours. However rapid cooling is achieved from about $50 \mathrm{~K}$ down to $8 \mathrm{~K}$. The cooling operates in a cyclical process, which is able to extract only a certain amount of heat energy in a cycle. Most materials, including the materials from which the cryostat is built show a rapid decline in heat capacity as temperature tends towards $0 \mathrm{~K}$. Thus, less heat extraction is required to lower the temperature in the $<50 \mathrm{~K}$ range and faster cooling is achieved. Although the rate of heat extraction can be expected to vary somewhat 
as the temperature difference between the reservoir and the sample decreases, this effect is comparatively small and the heat capacity effect dominates the rate of temperature change.

The last significant point of difference between the systems is the helium cryostat operates under high vacuum, whereas the Linkam operates at ambient pressures. The pressure in the helium cryostat is lowered using two pumps in series; a diaphragm pump for rough pumping, and a small turbo pump. The operating pressure is in the order of $1 \times 10^{-5}$ Torr. Taking Raman spectra of a sample in vacuum is advantageous, in that one will measure no nitrogen (or other atmospheric) lines in the Raman spectra. These lines are typically weak, but when one is measuring a weakly scattering sample, as is the case in the following experiments, then the removal of these lines is of great benefit to the interpretation of the spectrum in the low wavenumber region. The Linkam cannot be run in high vacuum because the window is not designed to handle high pressure differentials. However, the atmospheric measurements can be advantageous in some circumstances as well. One does not have to use air to fill the cryostat chamber. During the course of measurements we have charged the chamber with pure nitrogen, helium, and argon gases. One can also flow gas through the chamber during experiments, which may be useful in the case of studying the reaction of a chemical to different gas conditions. It is also, of course, possible that a material may change directly in response to a low pressure environment, making low pressure measurements impossible. Materials may evaporate or sublimate, for example.

For most of our experiments, the deciding factors are the accessible temperature range, and the greater throughput of the microscope objectives. Accordingly, the Linkam is used except in circumstances where low frequency or low temperature measurements are required. 


\section{Chapter 4}

\section{Results - BNT-BT}

This chapter reports the results of Raman experiments made on BNT-BT ceramics. BNT-BT is a lead free ferroelectric, so it has technological interest, and it is a bulk material which makes it easily accessible to experiments. Raman spectroscopy measurements of this material were absent from the literature, so it was therefore a good material to begin measurements on, as an easy experiment for which we could make a valuable contribution. I collected Raman spectra of this material and examined them for signals of a structural phase transition in the material, and the implications are discussed. The findings of this section were published in 2010 in [4]. It must be pointed out that some of the data presented in this section was collected prior to the beginning of the Ph. D. programme. The most important contribution made to this work as part of the Ph. D. was the poling study, section 4.3 .

A list of samples used in this chapter may be found in section 3.1.1, and the experimental method used in section 3.2.1.

\subsection{Raman study}

We conducted a study of the BNT-BT system by Raman spectroscopy to find out more about the structural phase transitions of BNT-BT, about which there had been some questions[62][63]. Raman measurements were missing from the 
literature on this material, and we felt that they could help resolve the outstanding questions.

We discovered that whereas bulk electrical measurements show a discontinuous change at about 350-400 K for $x>6 \%$, there is no sign of a discontinuous change at this (or any nearby) temperature in the Raman spectrum. This contrasts to the case for $x<5 \%$, for which both Raman and bulk electrical measurements show a discontinuous change.

Fig. 4.1 summarises these results. In the figure is shown the Raman spectra of two samples at near $100 \mathrm{~K}$ : BNT-5\%BT and BNT-6\%BT, and the same two samples at $400 \mathrm{~K}$. The key features of the low temperature $5 \%$ spectrum are: Peaks at $140 \mathrm{~cm}^{-1}$ and $290 \mathrm{~cm}^{-1}$, and a doublet peak at $530 \mathrm{~cm}^{-1} / 590 \mathrm{~cm}^{-1}$. In the case of the $6 \%$ sample, the $140 \mathrm{~cm}^{-1}$ peak is at lower frequency $120 \mathrm{~cm}^{-1}$ and has decreased in intensity; the 290 peak has split into a doublet at $260 \mathrm{~cm}^{-1} / 320 \mathrm{~cm}^{-1}$, the lower half of the doublet at $530 \mathrm{~cm}^{-1}$ has increased in intensity; and the upper half is at higher frequency $620 \mathrm{~cm}^{-1}$. The former spectrum shows BNT-BT in rhombohedral symmetry, and the latter in tetragonal symmetry. The exact same spectral changes are seen upon raising the $5 \%$ sample to $400 \mathrm{~K}$ - the softening of the $140 \mathrm{~cm}^{-1}$ mode, the splitting of the $290 \mathrm{~cm}^{-1}$ mode and the hardening of the $590 \mathrm{~cm}^{-1}$ mode. Note that the modes are all also broader, corresponding to the increased temperature. The high temperature $6 \%$ spectrum shows almost identical features, including the broadening, and this shows the two samples have the same local structure at this temperature. I also provide a more detailed view of the spectra of the $5 \%$ sample going through the phase transition in Fig. 4.2

\subsection{Phase diagram}

Our interpretation of these results is that the tetrahedral phase has a long range polar coherence at temperatures below the electrical phase transition. Near the electrical phase transition, the size of coherent ferroelectric domains shrink as the temperature rises and the moments cancel out in average. The structural symmetry however, does not change over this region - there is no accompanying structural phase transition. Starting from the pure BNT, $0 \mathrm{~K}$ point in the phase diagram, 


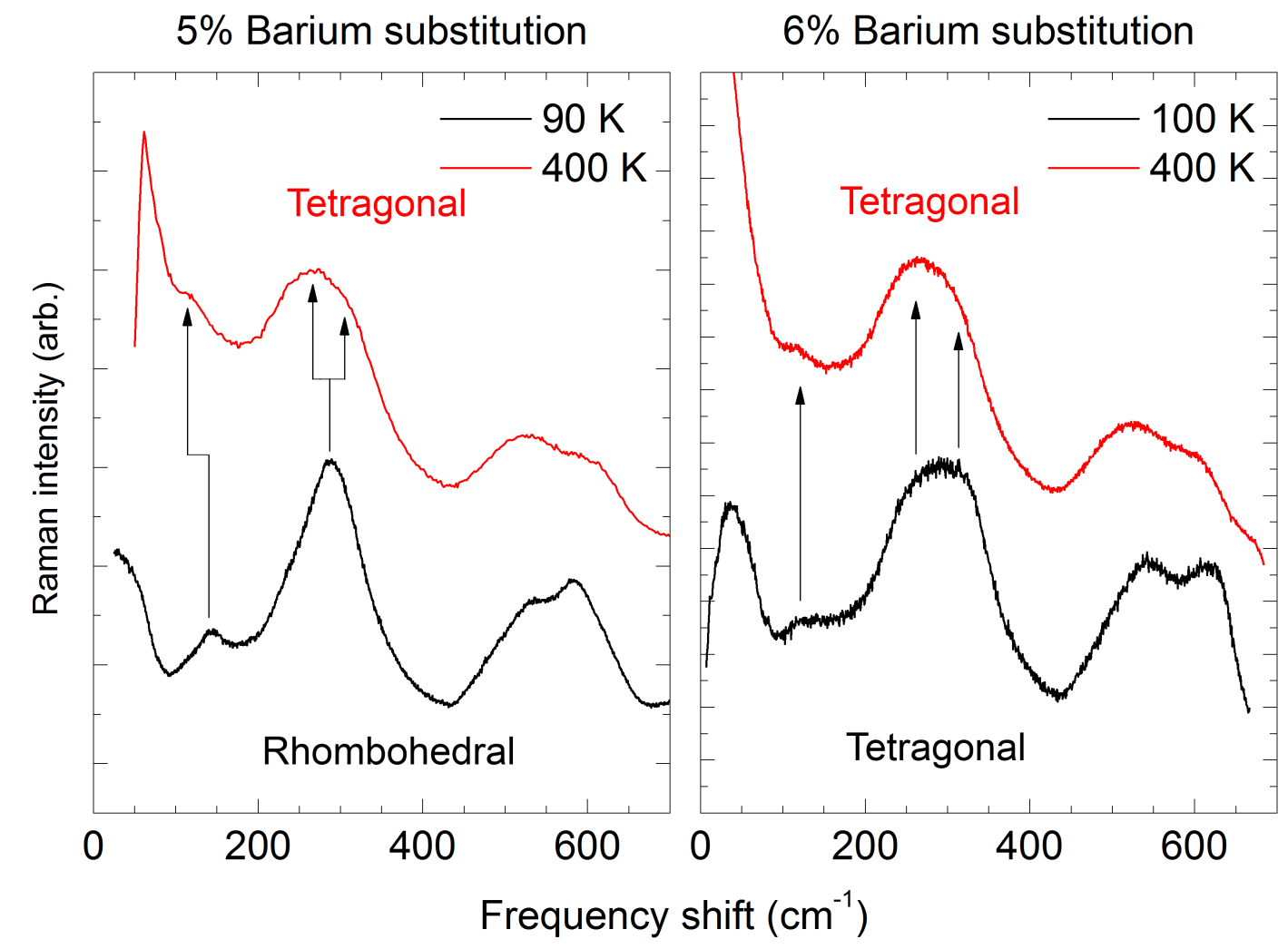

Figure 4.1: Spectra of BNT-BT taken by the author in a previous study. The spectra have been vertically offset for ease of viewing. The top (red) spectra are taken at high temperatures, and the bottom (black spectra) are taken at low temperatures. Left: 5\% Ba-substituted BNT, Right: 6\% Ba-substituted BNT. The arrows show features in the spectra including a discontinuous drop in the frequency of the $150 \mathrm{~cm}^{-1}$ mode, and the splitting of one peak into two near $300 \mathrm{~cm}^{-1}$. The spectra show no phase transition from low to high temperature in the $6 \% \mathrm{BT}$ sample, in opposition to what was found for bulk dielectric measurements. 


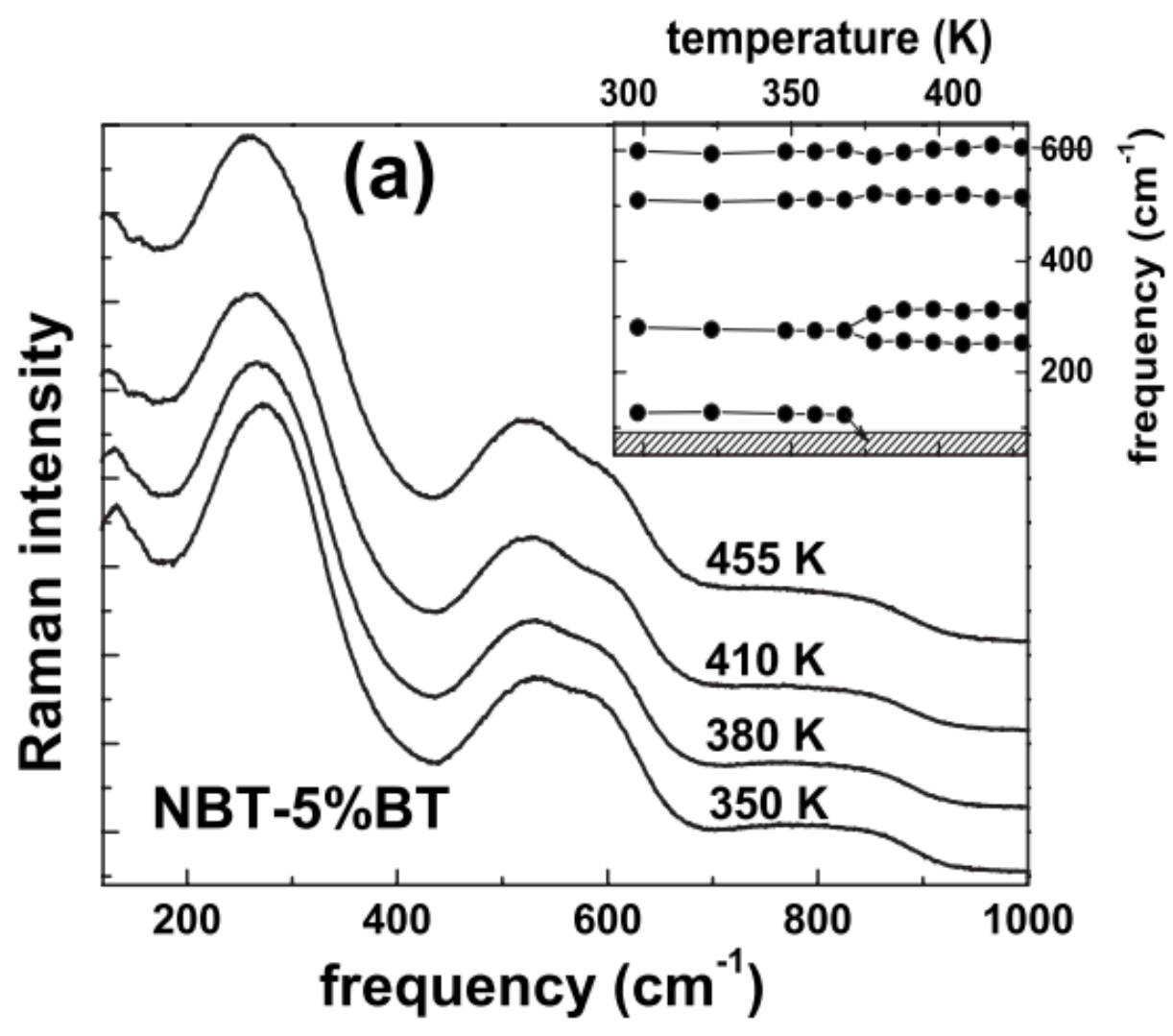

Figure 4.2: Temperature-dependent spectra of BNT-BT taken by the author in a previous study. The spectra have been vertically offset for ease of viewing. The spectra show the $5 \%$ BT sample being taken up in temperature over the phase transition between rhombohedral and tetragonal symmetries. Inset: Fitted peak frequencies of the Raman modes seen in the spectra. A discontinuous change can be seen in these at $380 \mathrm{~K}$. 
either increasing the concentration of BT above $5 \%$ or increasing the temperature above $375 \mathrm{~K}$ will transform the material from rhombohedral into tetragonal geometry, both cases in a first-order phase transition. This phase extends above and to the right of the rhombohedral phase, and eventually after further increases in temperature there is a transition to cubic structure. This explanation is consistent with $\mathrm{x}$-ray diffraction (XRD) studies which do measure a discontinuous change in this region of the phase diagram: with the domains small and omnidirectional enough to result in a null macroscopic polarization, the long-range average signal that would be picked up by XRD would appear different to that of an ordered configuration of domains. The different results are reconciled as Raman is a probe of local structure[64], whereas XRD is a probe of structure over longer length scales. Using Raman spectroscopy, we were able to discover the nature of the phase transition between the ferroelectric and non-ferroelectric regions of $x \geq 6 \%$ BNT-BT (as marked in the Takenaka phase diagram) is not displacive, but order-disorder driven. The change in electrical response occurs not because of a change in the crystal structure in the material, but because coherent long rage polar order is lost over this transition.

Fig. 4.3 Shows the phase diagram of BNT-BT reflecting our Raman spectroscopy results. Open points are step changes in electrical measurements of our BNT-BT samples, closed points are step changes in the Raman spectra. There is an electrical phase transition in high BT samples above $350 \mathrm{~K}$, as shown by the empty points. However, there is no structural phase transition accompanying it as shown by the absence of filled circles. Structurally, the phase marked Zone III in this diagram, and marked as antiferroelectric 'AF' in Takenaka's diagram is the same phase. The point at $350 \mathrm{~K}$ and $5,5 \%$ Barium substitution is therefore not a structural triple point.

This research suggests that we may be missing part of the picture in attributing the remarkable piezoelectric properties of PZT to an MPB on its own. It is a result supporting the conclusion of another recent paper studying a different ferroelectric system: that the termination of a MPB at a tricritical point between ferroelectric rhombohedral, ferroelectric tetragonal and paraelectric cubic is a crucial factor in the enhancement of $\mathrm{d}_{33}[65]$. A tricritical point of this kind is present in PZT, and also in the new material studied by this paper which resulted in a similarly strong enhancement of $\mathrm{d}_{33}$ as that found in PZT - but not in the BNT-BT system 


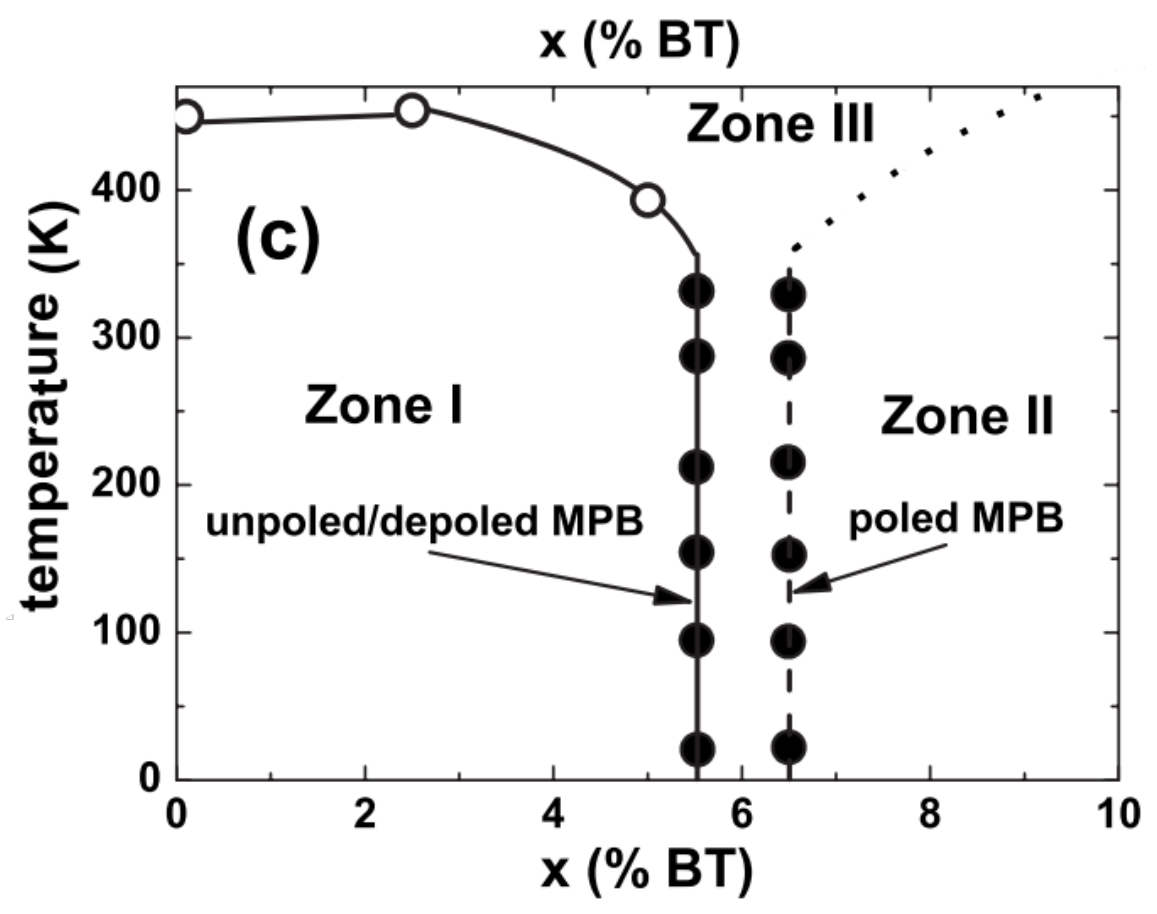

Figure 4.3: Phase diagram of BNT-BT based on Raman measurements of our samples. Zone I is ferroelectric and rhombohedral, zone II is ferroelectric and tetragonal, zone III is tetragonal, but is not ferroelectric. (Open symbols measure phase transitions as a function of temperature, closed symbols show implied phase transitions - 5\% sample is Rhombohedral and 6\% sample is Tetragonal at this temperature). Note the lack of any Raman signal of a phase transition between zones II and III, and contrast this with Fig. 2.1, the phase diagram in Takenaka et al. [16]. There is no structural change accompanying this electrical phase transition. 
according to the Raman data. A tricritical point would in theory create an even flatter free energy landscape than an MPB, as not two but three different structures coexist under those conditions at near-identical energies, granting access to a huge range of states to mediate polarisation rotation.

Further to the above result, the research proves the value of Raman spectroscopy to the study of ferroic phase transitions. Until the result was published, it was widely thought that each of the phases observed by Takenaka corresponded to a different structural phase. This shows the importance of measuring directly the local structure rather than bulk average structure, and Raman spectroscopy is an excellent tool for making these types of measurement.

As bulk dielectric measurements that suggested a phase boundary between the ferroelectric tetragonal region and the anti-ferroelectric region are carried out on samples that have been electrically poled, due diligence required that we also carried out Raman experiments on similarly poled samples. We sought to find out if the Raman spectra of the poled samples show a structural phase transition or not. If so, it would suggest a change in the structure is caused by poling, which would also be an interesting result.

\subsection{Poling study on BNT-BT}

Samples of BNT-BT produced the same way as the samples used above were then poled in an electrical field - see section 3.2.1

The 5\% Ba-substituted poled sample showed no significant changes from the non-poled samples in their Raman spectra, and in fact show no differences across the temperature range accessible to this apparatus. Similarly, samples with $x<5 \%$ showed no difference. However, the $6 \%$ sample showed dramatic differences. At ambient temperatures, and when cycled down to $80 \mathrm{~K}$, the sample displays a single peak at $280 \mathrm{~cm}^{-1}$ rather than a doublet, the $600 \mathrm{~cm}^{-1}$ peak is not hardened and the low frequency peak is not softened; its spectrum is strikingly similar to the rhombohedral spectrum, and we conclude that the act of poling has shifted the sample into the rhombohedral phase. Fig. 4.4 shows spectra the poled $6 \%$ sample 


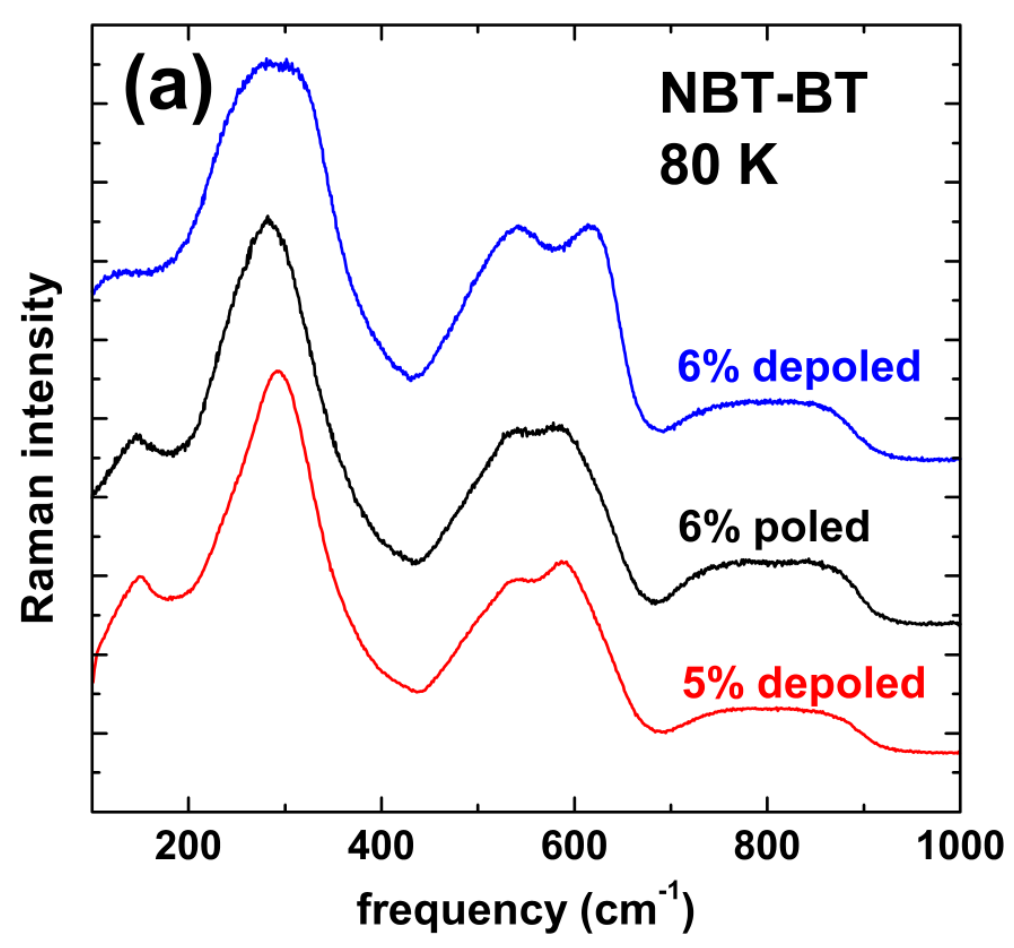

Figure 4.4: Raman spectrum of the poled $6 \%$ sample compared with spectra of the depoled samples at 5\% (Rhombohedral) and 6\% (Tetragonal). The spectra have been vertically offset for ease of viewing. The poled sample is dissimilar to the depoled sample of the same composition. Reprinted figure with permission from[4]. Copyright 2010 by the American Physical Society.

of BNT-BT compared with the spectra of depoled $5 \%$ and $6 \%$ BNT-BT measured at room temperature - note that the spectrum of the poled sample looks more similar to the $5 \%$ unpoled spectrum than to the $6 \%$ unpoled spectrum. A closer examination of the poled spectrum shows that there is a broadening of the peaks compared to the unpoled samples, indicating that the poled sample is in a mixed phase. Fig. 4.5 shows results of fitting the poled spectrum to a weighted sum of the $5 \%$ and $6 \%$ unpoled samples, suggesting that there is about a $20 \%$ contribution by the tetragonal phase in the poled sample.

Upon heating the sample above $375 \mathrm{~K}$, the poled sample goes through the rhombohedral-tetragronal phase transition in the same way the $x<5 \%$ samples do; the $280 \mathrm{~cm}^{-1}$ peak spits into two, the $600 \mathrm{~cm}^{-1}$ peak hardens and strengthens and the low frequency peak softens. Fig. 4.6 shows dielectric permittivity measurements of the poled samples compared with the unpoled ones (these measurements not my contribution). At low temperatures, the unpoled samples show a clear fre- 


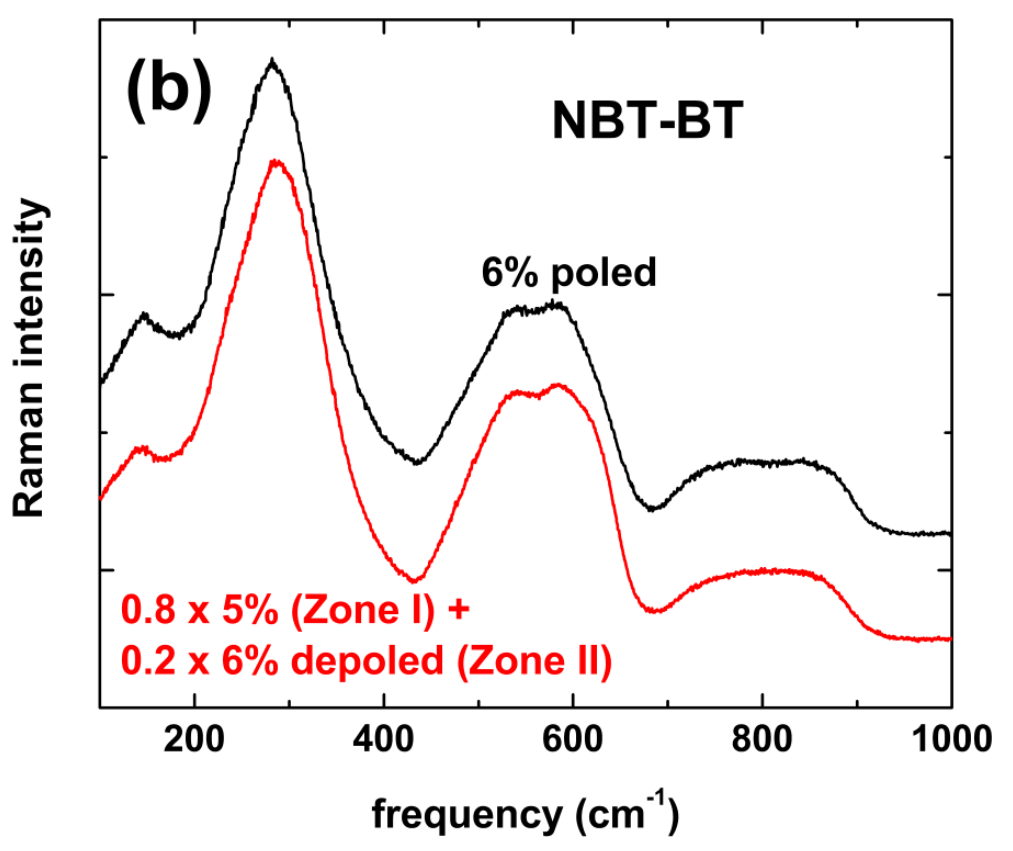

Figure 4.5: Raman spectrum of the poled $6 \%$ sample compared to a line created from a $80 / 20$ mix of spectra of depoled samples. The spectra have been vertically offset for ease of viewing. This suggests that the $6 \%$ poled sample is in a mixedphase. Reprinted figure with permission from[4]. Copyright 2010 by the American Physical Society. 
quency dispersion, but the poled samples are independent of frequency. However, at $375 \mathrm{~K}$, the same temperature as we observe the phase transition in the Raman spectra, the lines for the different frequencies split apart, matching the unpoled sample. The BNT samples show a peak in permittivity at just under $600 \mathrm{~K}$. this peak corresponds to the paraelectric to anti-ferroelectric phase transition, above the phase transition we are investigating in this study.

On return to room temperature, the rhombohedral phase is never recovered, as measured by the Raman spectra. The sample was tested again after several days to be sure, as it takes the $5 \%$ (depoled) sample a day or so to completely revert to the rhombohedral phase after being cycled above the transition temperature.

\subsection{Effect of poling on the phase diagram}

The obvious interpretation is that cycling the poled $6 \%$ sample above $375 \mathrm{~K}$ depoles the sample, and subsequently the equilibrium phase of a $6 \%$ substituted sample is returned to tetragonal, so no transformation back to rhombohedral is observed. Seen in another way, the potential energy of this system contains a term describing the interaction of the dipole moments (the self-dipole term). This term favours the rhombohedral, ferroelectric phase (refer to Fig. 2.1). When the dipole moments are lined up, which can be caused by poling, this term is large. In the $6 \%$ sample, the energy difference between tetragonal and rhombohedral is small and this is sufficient to change the equilibrium phase at room temperature of the material to rhombohedral. Now in the rhombohedral phase, the poling order of the material is more stable, and results in a higher remnant polarisation once the external field is removed. The energy contribution at this point is still large enough that the equilibrium phase is rhombohedral. Finally, given enough thermal energy the order of the dipole moments will be destroyed, randomising the directions and lowering the contribution of the self-dipole term to zero. When the material is subsequently lowered in temperature, the self-dipole term remains zero, so the equilibrium phase is back to being tetragonal, and no phase transformation back to rhombohedral occurs.

The $7 \%$ and higher poled samples also had identical temperature-dependent 


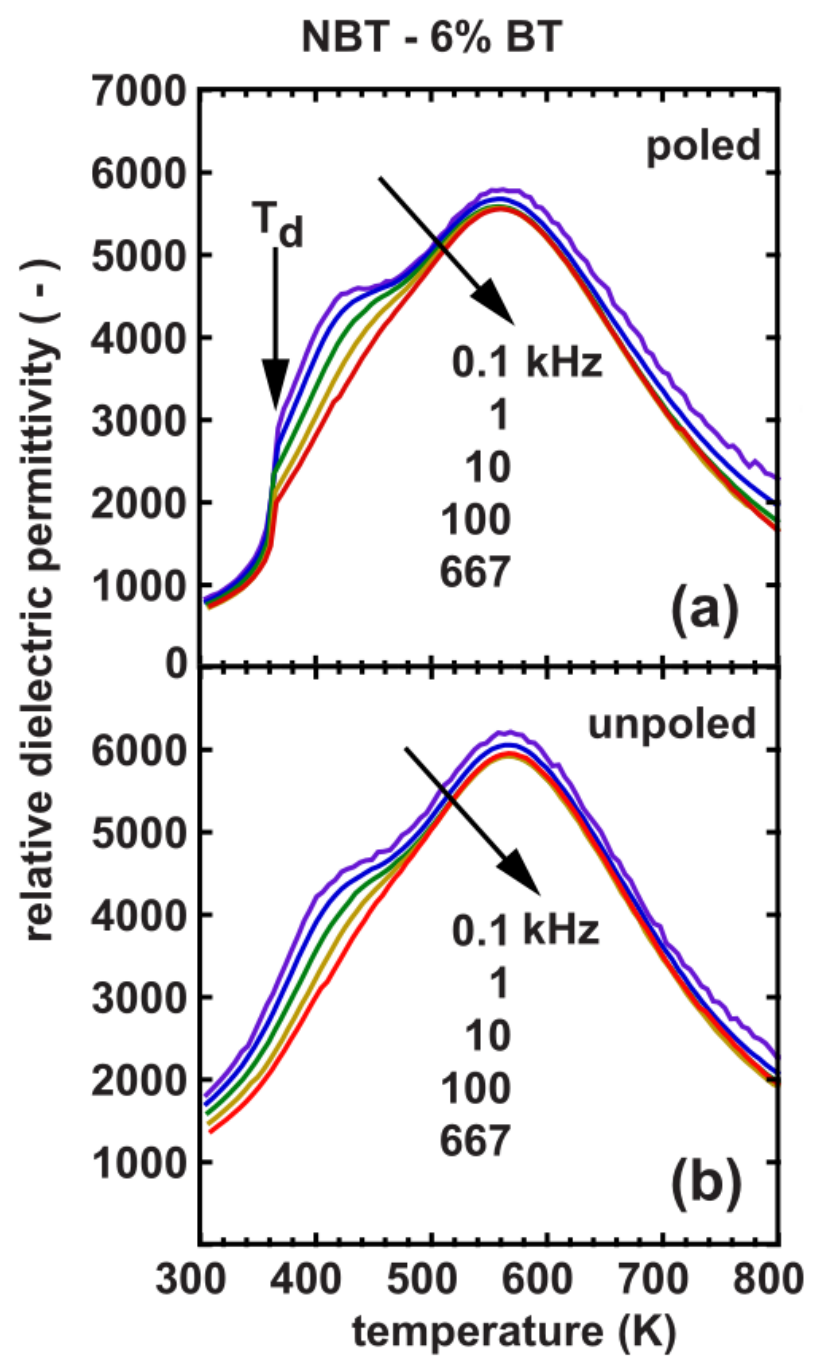

Figure 4.6: Dielectric permittivity of the $6 \%$ samples - poled and depoled. A temperature of depoling is clearly seen and is marked. Below this temperature, there is a notable difference in the permittivity for poled and unpoled samples. Reprinted figure with permission from[4]. Copyright 2010 by the American Physical Society. 
Raman spectra to their unpoled versions, so it can be seen that poling the samples has significant effects only very close to the morphotropic phase boundary, where the phase of the BNT is most unstable. The poling stabilises the rhombohedral phase in favour of the tetragonal one, shifting the phase boundary to the right by $1 \%$ or so. The unstable nature of BNT-BT near this morphotropic phase boundary indicates that there is indeed a significant flattening of the free energy near this boundary. This facilitates easy switching between polarization directions, and is the source of the enhancement of the piezoelectric response. However, as noted in the earlier result, the morphotropic phase boundary does not terminate in a triple point, as PZT does.

The fact that the spectra we measured showed a mixed phase might be significant, but it is probably not. At least one obvious explanation for the observed phase mixing exists: It is reasonable to expect that at some substitution level without poling, the material would also be in a mixed phase. That we did not detect this in the unpoled sample could be explained by the fact that we only have coarse variation in composition, $1 \%$ steps, and the shift of the MPB in the phase diagram to the right is known to be a shift of less than $1 \%$. It is reasonable to interpret that the shift put the region of mixed phase in the phase diagram over the exact composition that we measured.

\subsection{BNT-BT conclusions}

The Raman data on BNT-BT that we collected showed that the electrical phase transitions in this system did not wholly correspond to the structural phase transitions in it. Although there is a step change in the electrical properties between phases designated as FE and tetragonal ferroelectric in previous work, both those regions of the phase diagram were shown to have the same structural phase. The electrical step change is caused by a loss of long-range order in the tetragonal ferroelctric phase.

In electrically poled samples, we find a comparative stabilisation of the rhombohedral phase over the tetragonal phase in samples near $6 \%$ substitution. The effect is weak, applying only very near to the morphotropic phase boundary, and 
furthermore is an inherently hysteretic effect, with thermal destruction of polar order sufficient to revert the material back to the unpoled phase diagram. Poling the samples does not affect the electrical step change discussed above.

The results suggest that a tricritical point is a crucial component in the highest piezoelectric enhancements found in PZT and other ferroelectrics; And that the low enhancement of BNT-BT compared to this and other systems can be explained by the absence of such a point in the phase diagram, and correspondingly a lessflat free energy landscape. Whereas before it was assumed that BNT-BT had a tricritical point such as this, the Raman spectra have proven that not to be the case. 


\section{Chapter 5}

\section{Results - PT Nanowires}

This chapter reports the results of experiments on the lead titanate nanowire samples. Raman spectroscopy and SEM images are obtained for this system. The wires were found to have an anomalously enhanced cubic-tetragonal structural phase transition temperature when their diameter is close to $100 \mathrm{~nm}$. TEM are subsequently taken of these samples (TEM not the contribution of the author). The images subsequently revealed a similar enhancement of the tetragonality in the wires, also diameter dependent and peaking at $115 \mathrm{~nm}$. A possible reason for this enhancement is discussed. A list of sample used in this chapter may be found in section 3.1.2, and the experimental method used in section 3.2.2.

\subsection{Sample $1->100 \mathrm{~nm}$ PT nanowires}

\subsubsection{Initial SEM imaging}

Initial Raman spectroscopy measurements were made on bundles of PT nanowires in order to establish collection parameters that would result in the highest possible throughput. Next, SEM images were taken of sample 1 to locate and identify single nanowires of a range of diameters that could be measured by Raman spectroscopy. Fig. 5.1 are SEM images showing the morphology of the wires. Wires on the substrate are typically clustered together into bundles, with a few wires of similar 
orientation stuck together on the substrate. Individual wires diameters on sample 1 varied from a little over $100 \mathrm{~nm}$ to around $1 \mu \mathrm{m}$, with lengths as much as tens of micron. Some wires also showed a divergence, with one stem splitting into several thinner branches. More rarely, a single wire was seen on the substrate (ie - not a bundle). Singular wires are preferred for Raman measurements, so that inhomogeneity of the wires will not affect the results.

SEM images d), e), and f) in Fig. 5.1 show the three wires that were measured by Raman spectroscopy in this study. They have diameters of $650 \mathrm{~nm}, 250 \mathrm{~nm}$, and $125 \mathrm{~nm}$ respectively. Note that the wires of $650 \mathrm{~nm}$ and $125 \mathrm{~nm}$ are not totally singular. In the case of the $650 \mathrm{~nm}$ wire, there are a few smaller wires present on the larger wire. However, the contribution from the large wire should hopefully dominate the spectrum. For the $125 \mathrm{~nm}$ wire, there are two wires that make up most of the bulk, and there are a few other wires besides. Although this is not ideal, this was the best example on the substrate of smallest-diameter wire by itself we were able to find. All of the wires present in this small bundle at least have similar diameter, so hopefully the spectrum still gives us a reasonable approximation of a single nanowire. In this case, the laser spot was directed to the upper part of the wire, although because of the size of the spot, part of the other wires would also have been illuminated.

\subsubsection{Raman features}

The Raman spectrum from the $650 \mathrm{~nm}$ thick wire at $100{ }^{\circ} \mathrm{C}$ is shown in Fig. 5.2. The lead titanate peaks are clearly visible, and there is a small peak at $414 \mathrm{~cm}^{-1}$ visible from the sapphire substrate. The size of this wire is large enough that most of the laser spot is filled with the wire, so signal to noise is strong in this case. This sample was taken up in temperature until the tetragonal-cubic phase transition was found, to give us an example of what to expect in the Raman spectra for this event. Fig. 5.3 shows the results. In these spectra, there is a narrow peak at $42 \mathrm{~cm}^{-1}$. This is a plasma line. Note that all the PT lines are weaker than at low temperature. The peak at $71 \mathrm{~cm}^{-1}$ (at $470{ }^{\circ} \mathrm{C}$ ) is the most prominent, and has the most significant behaviour. The peak shows a dramatic softening as the temperature is raised from $470{ }^{\circ} \mathrm{C}$ to $500{ }^{\circ} \mathrm{C}$, dropping from $71 \mathrm{~cm}^{-1}$ to $54 \mathrm{~cm}^{-1}$ at 


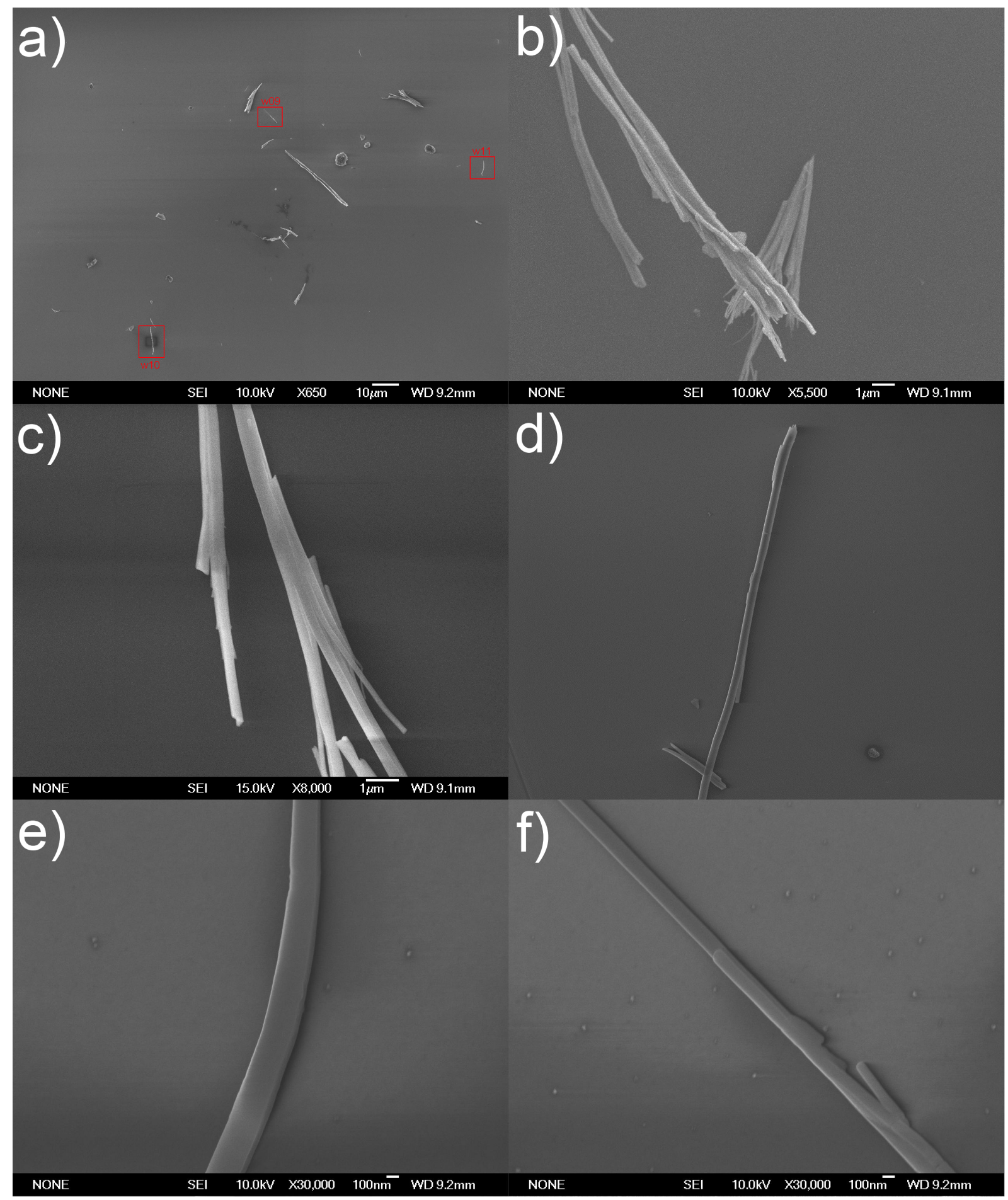

Figure 5.1: SEM images of PT nanowires showing morphology. The wires shown in d) e) and f) were used to obtain Raman spectra and have diameters of $650 \mathrm{~nm}$ $250 \mathrm{~nm}$ and $125 \mathrm{~nm}$ respectively. 


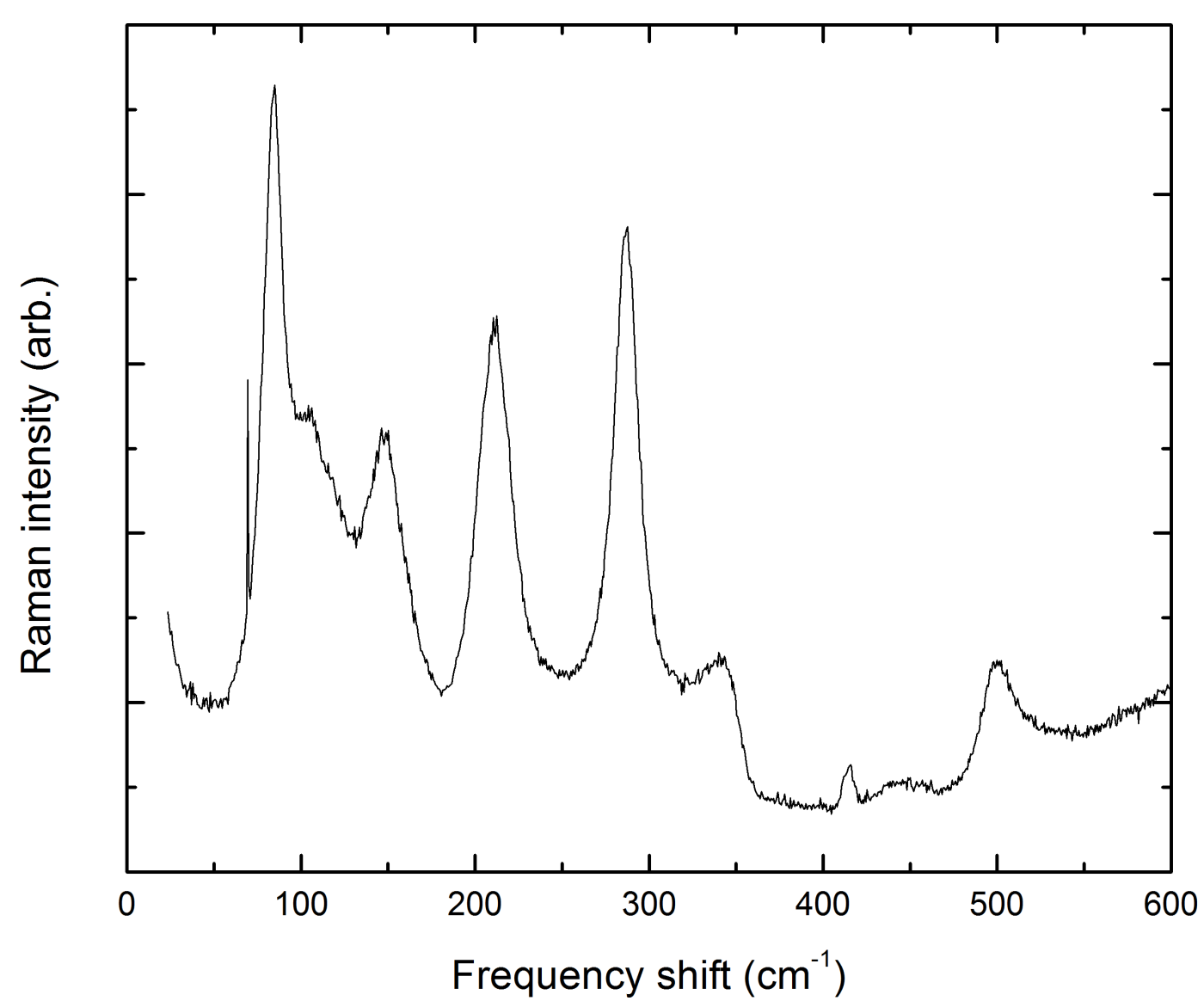

Figure 5.2: Raman spectrum of a large PT nanowire used in this sample. The first order peaks are clear in the tetragonal, ferroelectric phase.

$495^{\circ} \mathrm{C}$ just before disappearing. The other PT peaks in the spectrum also weaken significantly between $495^{\circ} \mathrm{C}$ and $500^{\circ} \mathrm{C}$. All these modes are Raman silent in the cubic phase, so the assignment of this spectral change as corresponding to the tetragonal-cubic phase transition is easy to make.

Fig. 5.4 shows the Raman spectra of the three different wires $-650 \mathrm{~nm}, 250 \mathrm{~nm}$ and $125 \mathrm{~nm}$ thick - at three different temperatures $-400^{\circ} \mathrm{C}, 500^{\circ} \mathrm{C}$ and $600{ }^{\circ} \mathrm{C}$. One can notice that the signal to noise ratio in the thinner wires is less in general than in the thicker wire, and that the signal to noise ratio also is inconsistent between measurements for the thinner wires. This variation is due to experimental difficulties of centering and holding the laser spot directly over the thinner wires, numerous readjustments of position needed to be made to keep the sample in the spot, and small differences in position would undoubtedly have occurred. It is also difficult to focus the microscope objective perfectly for the thinner wire 


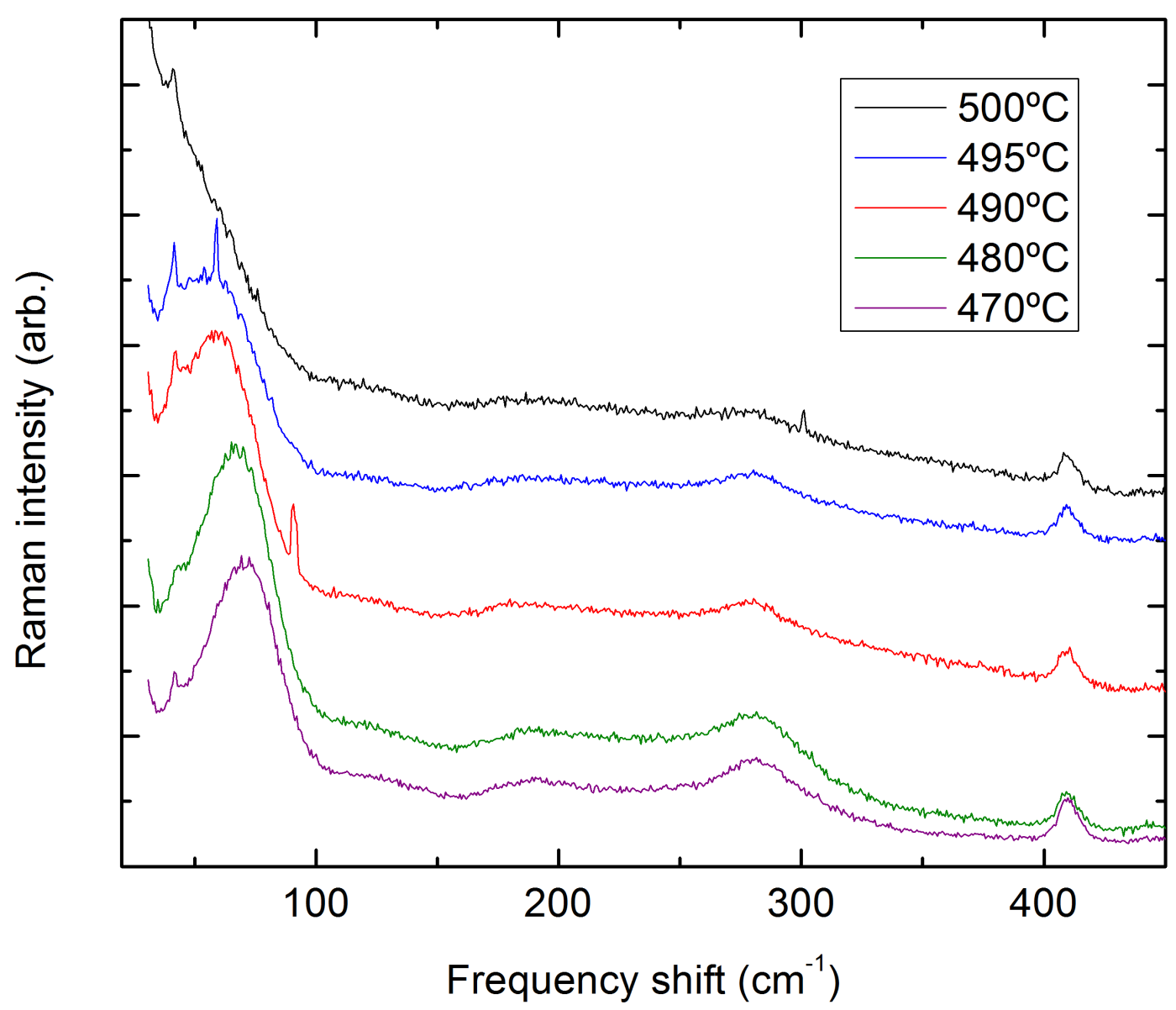

Figure 5.3: Temperature dependent Raman spectra of a large PT nanowire. The spectra have been vertically offset for ease of viewing. This wire shows the tetragonal-cubic phase transition at $500{ }^{\circ} \mathrm{C}$, the same as bulk PT. 
measurements. Usually one can achieve optimum focus by collecting spectra for a few seconds and adjusting until maximum Raman signal is attained, but in these cases the signal from the sample is too weak to make a noticeable peak in a recording of a few seconds. As a consequence, it is quite likely that the collection conditions are not ideal, and not $100 \%$ consistent. This can be seen in the spectra. This makes it difficult to use the amplitude of peaks as a reliable indicator for structural changes in the wires. However, the focusing and positioning imperfections will not affect the frequency of the peaks measured, so these can still be considered reliable. We use the frequencies - in particular, the softening of the lowest frequency mode - as our indication of the phase transition. The softening behaviour of the vibrational modes in bulk PZT is well established[66].

At $400{ }^{\circ} \mathrm{C}$, all three wires are clearly in the tetragonal phase. At $500^{\circ} \mathrm{C}$, the $650 \mathrm{~nm}$ wire has lost almost all of the intensity of the tetragonal peaks, and displays predominantly the cubic Raman spectrum, and the $250 \mathrm{~nm}$ wire, while still in the tetragonal phase shows a pronounced softening in the low frequency peak. The $125 \mathrm{~nm}$ wire remains tetragonal, with minimal softening shown. At $600{ }^{\circ} \mathrm{C}$, both the $650 \mathrm{~nm}$ and $250 \mathrm{~nm}$ wire have lost their tetragonal modes and show predominantly the cubic spectrum, but the $125 \mathrm{~nm}$ wire is still quite clearly in the tetragonal phase, with some softening of the low frequency mode.

\subsubsection{Temperature dependence and phase transition}

The low frequency region of the spectra of all three samples was fitted to two Lorentzian lines using a least-squares fitting method. One peak modeled the soft mode, while the other peak modeled the background with the central frequency noise. The results of the peak fitting are displayed in Fig. 5.5. The points are the central frequencies of the Lorentzians fitted to the experimental data. The cubic to tetragonal transition temperature is $500^{\circ} \mathrm{C}$ in the $650 \mathrm{~nm}$ wire, which is the same behaviour as the bulk material. It is raised to about $530^{\circ} \mathrm{C}$ in the $250 \mathrm{~nm}$ wire. In the $125 \mathrm{~nm}$ wire, it is still in tetragonal at $600{ }^{\circ} \mathrm{C}$ as mentioned above. The $125 \mathrm{~nm}$ wire shows noticeably broader peaks than the larger wires. $600^{\circ} \mathrm{C}$ is the upper limit of the temperature control apparatus, so we can only project a transition temperature. A simple square-root fit to the data predicts a transition 


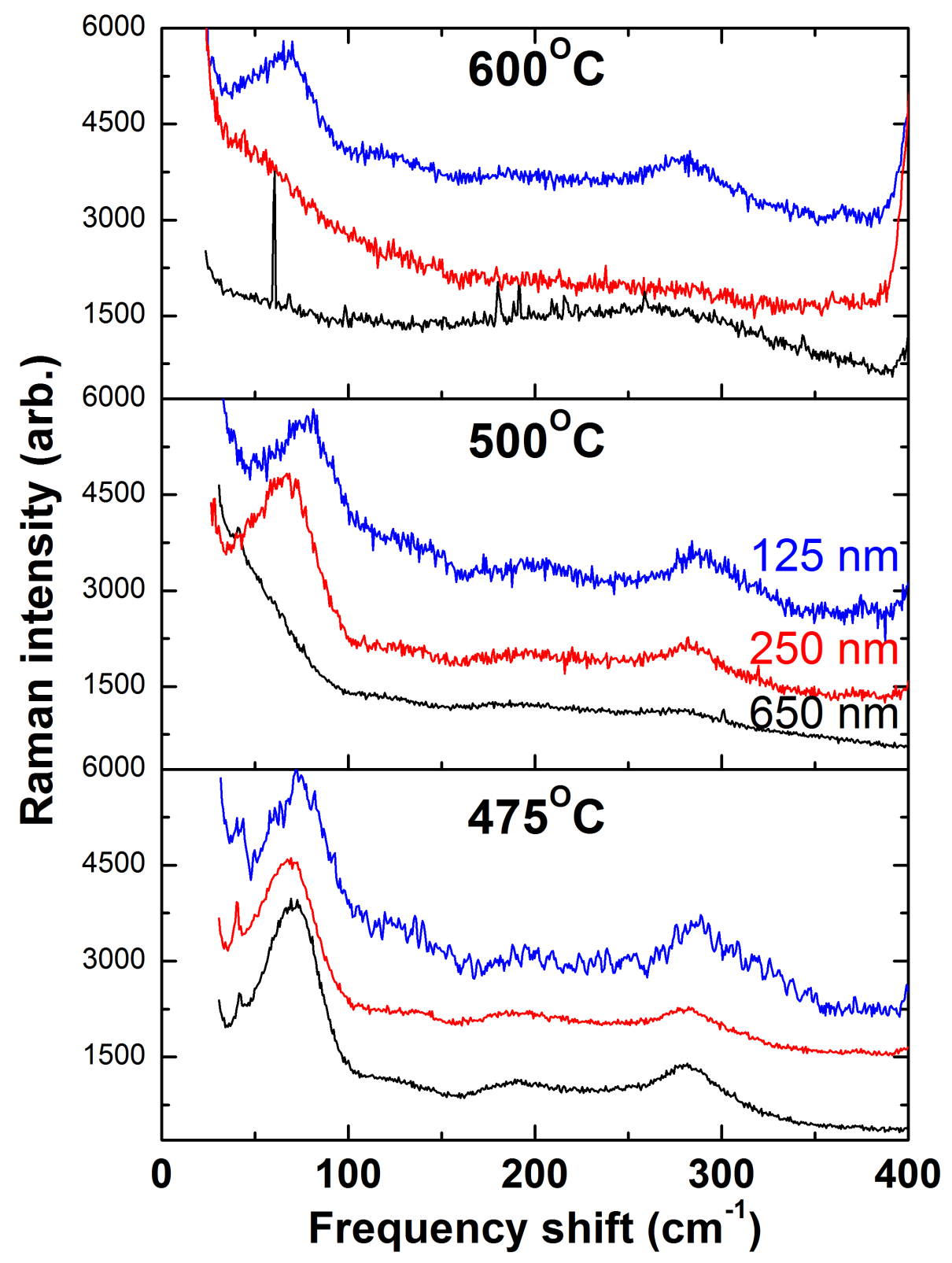

Figure 5.4: Raman spectra of three wires at three temperatures. The spectra have been vertically offset for ease of viewing. In blue: $125 \mathrm{~nm}$ wire, red: $250 \mathrm{~nm}$ wire and black: $650 \mathrm{~nm}$. At $475{ }^{\circ} \mathrm{C}$ all three wires are in the tetragonal phase. at $500{ }^{\circ} \mathrm{C}$, the smaller two wires are tetragonal and the largest wire is cubic. At $600^{\circ} \mathrm{C}$, the larger two wires are cubic and only the smallest wire is still in the tetragonal phase. 
of about $675^{\circ} \mathrm{C}$. It should be noted that this projection is very rough - it is based on a noisy data set which does not show sharp frequency variation over the region we have data for. However, even a $100{ }^{\circ} \mathrm{C}$ increase in transition temperature which we do have unambiguous measurements for - should be seen as a hugely significant change in structural dynamics from the bulk case.

So the increase in transition temperature that we measure in these wires is strongly dependent on the diameter of the wires. On this sample however, we were unable to find any wires under $100 \mathrm{~nm}$ to measure by Raman spectroscopy.

\subsection{Sample $2-<100 \mathrm{~nm}$ PT nanowires}

\subsubsection{Initial SEM imaging}

A sample of PT nanowires dispersed with a higher dilution on a different substrate was studied in a similar manner. On this sample, SEM imaging was done prior to any Raman measurement or temperature changes. The imaging was able to pick up a multitude of nanowires smaller than $100 \mathrm{~nm}$. Figs. 5.6 and 5.7 show preliminary SEM images of these regions of wires. In part a) of Fig. 5.6, a red circle shows the location where we directed the Raman laser spot in subsequent measurements. part a) of Fig. 5.7 shows a higher zoom image of this area. For these SEM images, the sample was not coated in a thin conductive layer. The images are therefore of lower resolution than is ideal, and we were unable to measure properly the diameter of these wires. High resolution SEM images would be taken after the Raman measurements, we just needed some rough images to tell us where to direct our laser spot. One can see the regions of nanowires in these images. The circle superimposed on the image is the approximate size of the laser spot for Raman imaging. The region shown was chosen as the best candidate to try and get a spectrum from.

The wires we were looking at now are very much smaller both in length and in width than the size of the laser spot of our Raman measurement. So measuring a single nanowire of this size would mean very little volume of PT was being excited, resulting in a weak signal. A region containing many sub $100 \mathrm{~nm}$ wires in 


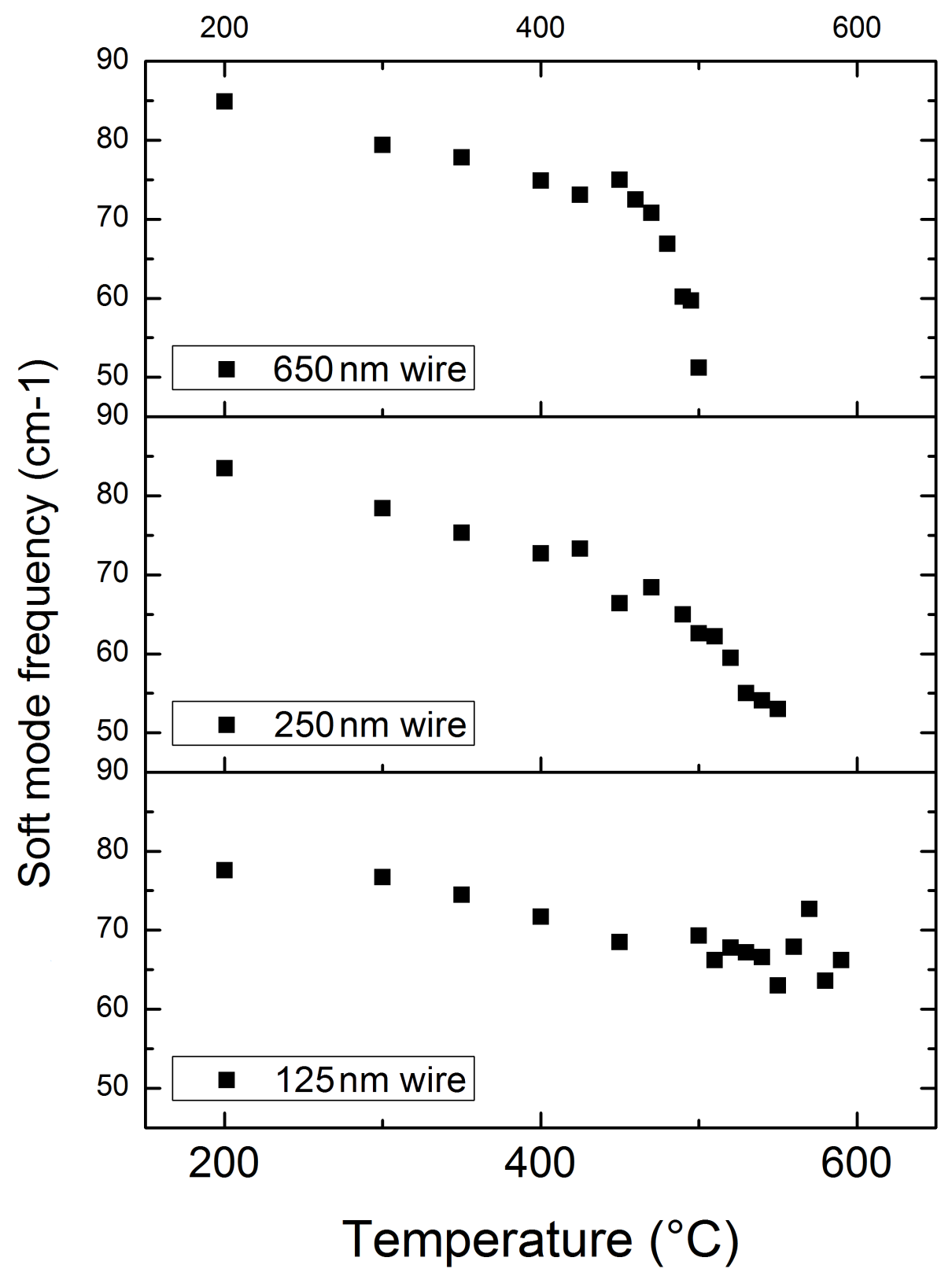

Figure 5.5: Temperature dependence of soft mode frequency of the three wires showing softening. Recall that the $125 \mathrm{~nm}$ wire is still tetragonal at the highest temperature measured. Its soft mode frequency at $600^{\circ} \mathrm{C}$ is still above the frequency at which the mode disappeared in the other wires measured. 
the space of a laser spot was therefore advantageous to increase the total scattering volume of PT we could address in the measurement. The spot chosen had no wires overlapping, which was a nice bonus. Several wires would be illuminated during a single measurement, and we do not have an accurate measurement of the diameter of the wires, but every wire in the measuring region would be of diameter well below $100 \mathrm{~nm}$. Our best estimate of the average diameter of nanowires in this region is $60 \pm 20 \mathrm{~nm}$. This figure was obtained from the SEM image in Fig. 5.7 part b.

\subsubsection{Raman spectra}

Fig. 5.8 shows raw Raman spectra taken in this spot. The measurements were far from trivial due to the weakness of the signal. However, with sufficiently long integrations (these samples were integrated for 10 minutes each) some PT peaks can be made out from the noise - both the random component and the low frequency component. The low frequency component is very pronounced in these measurements - the slit width had to be increased in order to achieve a reasonable strength of signal, so the laser line itself was broadened and it dominates the spectrum at low frequency.

First of all, the small sharp peak at $87.5 \mathrm{~cm}^{-1}$ in all the spectra is a plasma line. The features at $377,415,450$ and $479 \mathrm{~cm}^{-1}$ are lines from the sapphire substrate. From the PT, the most easily seen peak is the $287 \mathrm{~cm}^{-1}$ peak. This can be seen in the raw spectrum at each temperature. In the clearer spectra at $300{ }^{\circ} \mathrm{C}$ and $400{ }^{\circ} \mathrm{C}$, the other PT features at about 85,150 and $210 \mathrm{~cm}^{-1}$ can be made out by eye. Fig. 5.9 shows a comparison of the spectrum at $450{ }^{\circ} \mathrm{C}$ to that at $500^{\circ} \mathrm{C}$. At $500{ }^{\circ} \mathrm{C}$, there is a big drop in overall intensity of the spectrum. Also significant is the complete disappearance of the PT line at $287 \mathrm{~cm}^{-1}$. The sapphire lines remain, and their intensity is not significantly changed from the $450^{\circ} \mathrm{C}$ case.

To better reveal the PT lines, a Lorentzian line was fitted to the central frequency signal in each spectrum, and the line subtracted from the raw data. The result is shown in Fig. 5.10. The plasma line has also been removed in these spectra, for ease of viewing. In these spectra, the signal to noise is very weak, but it is much easier to pick out the PT features. The soft mode can be seen softening in 


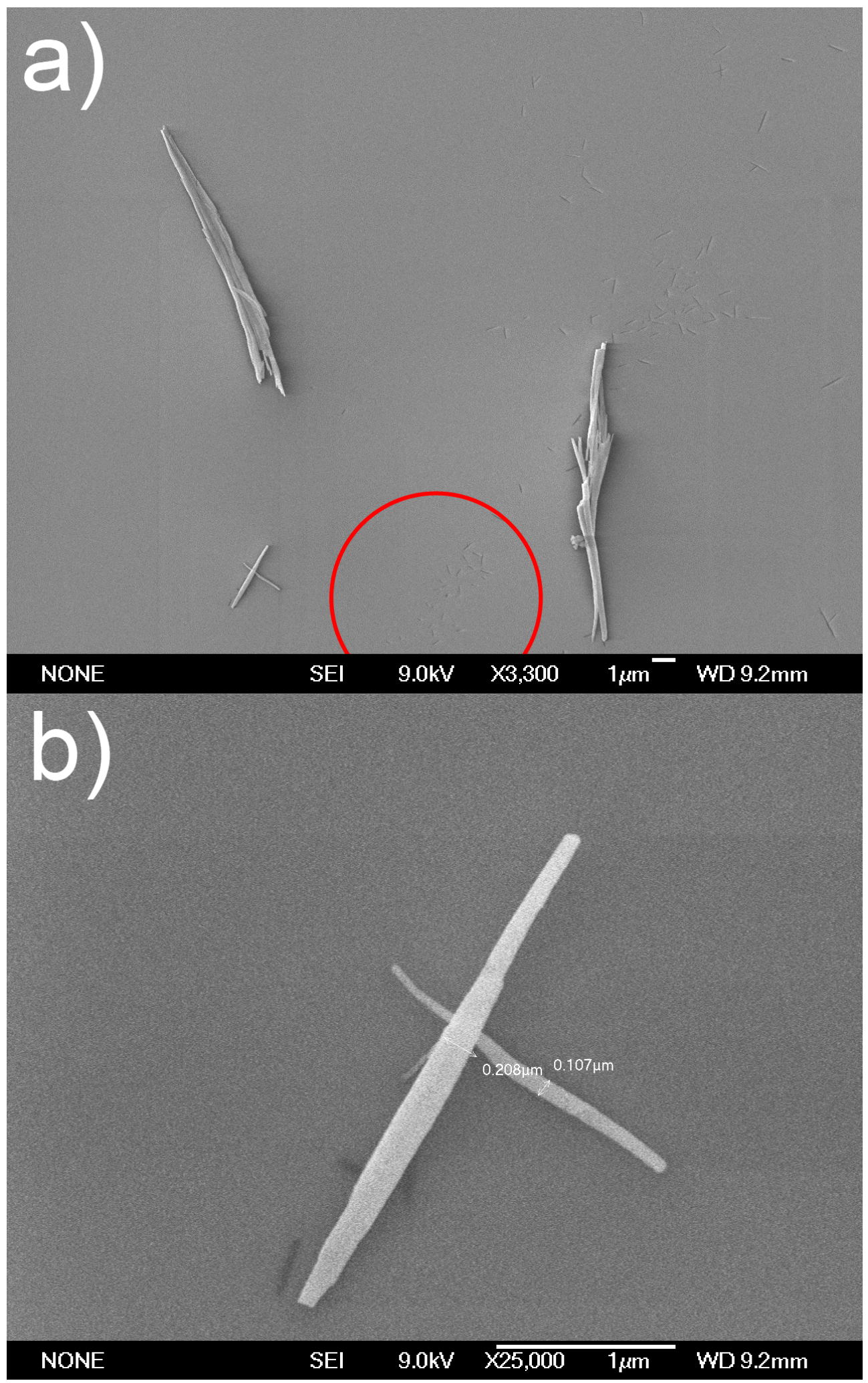

Figure 5.6: SEM images showing the smallest wires studied. A red circle in part a) shows on this figure the location and size of the laser spot used to make Raman measurements b) measurements made on the crossed wires can be used to make comparisons for the insufficiently resolved wires. 


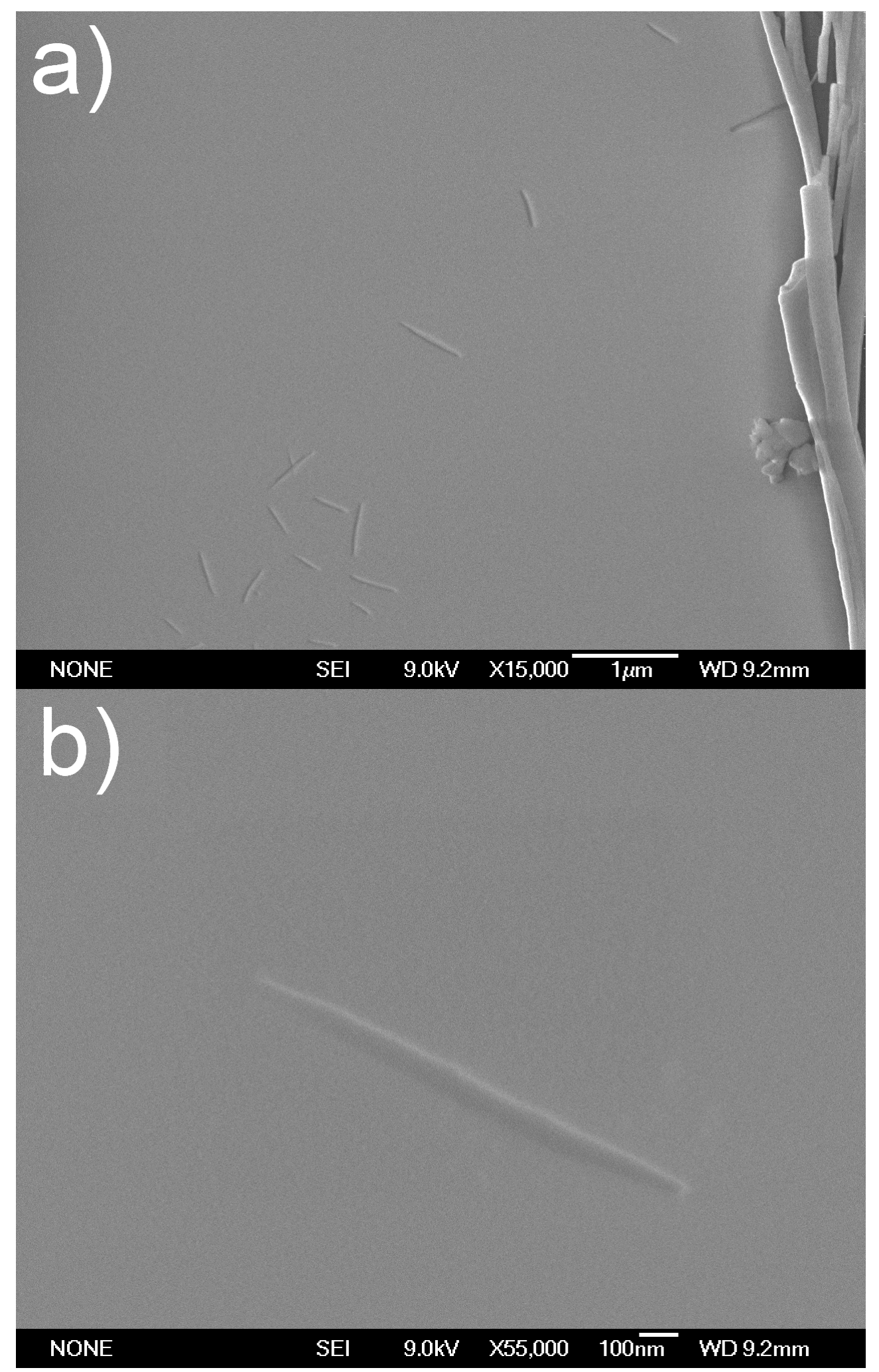

Figure 5.7: SEM images showing the smallest wires measured. These are the highest resolution images of the wires measured prior to measurement. 


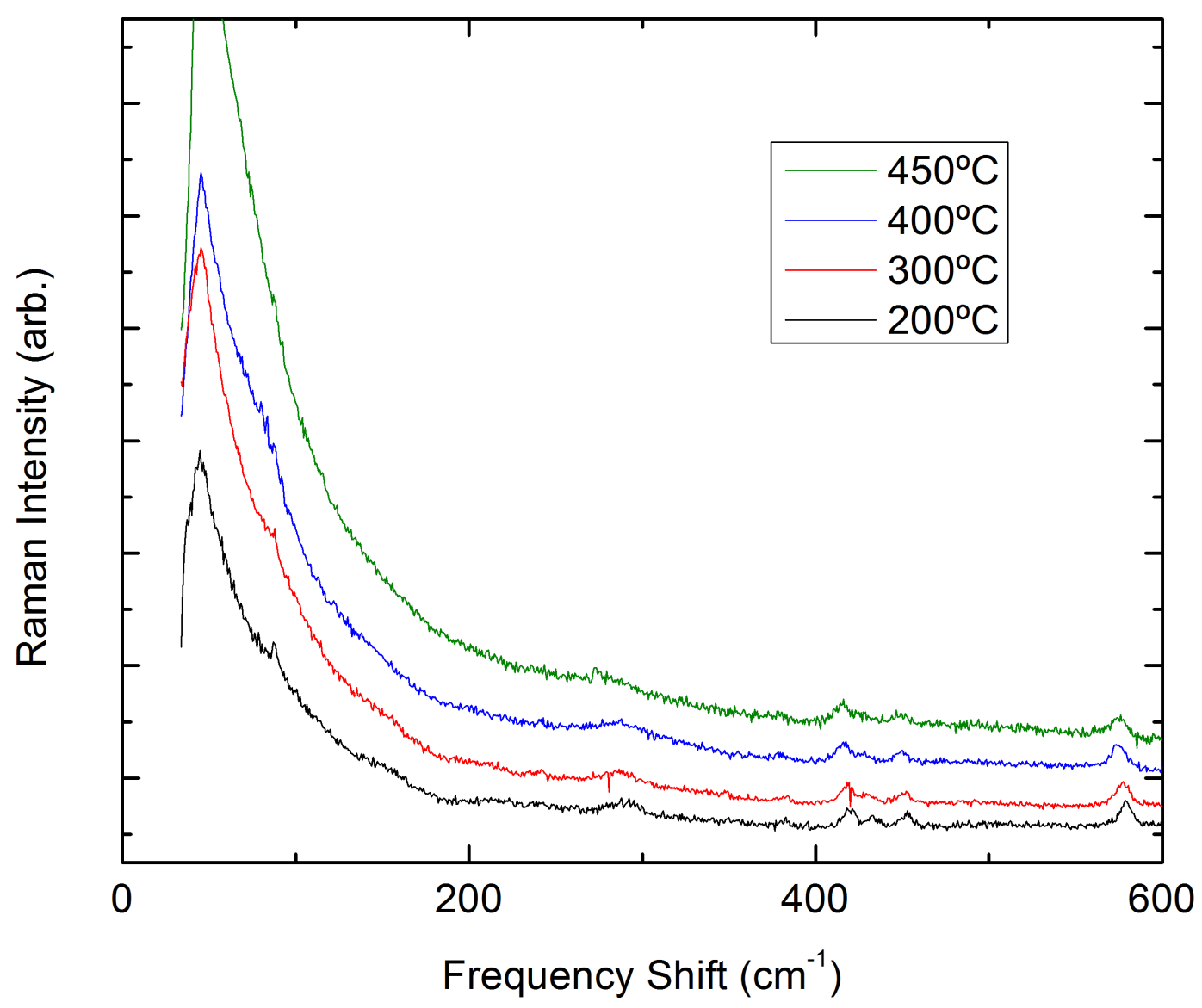

Figure 5.8: Raw temperature dependent Raman spectra collected from the region of sub $100 \mathrm{~nm}$ PT nanowires. The spectra have been vertically offset for ease of viewing. The spectra are dominated by the central frequency noise. The features above $400 \mathrm{~cm}^{-1}$ are sapphire lines from the substrate, and PT peaks can be seen at 85 and $287 \mathrm{~cm}^{-1}$. 


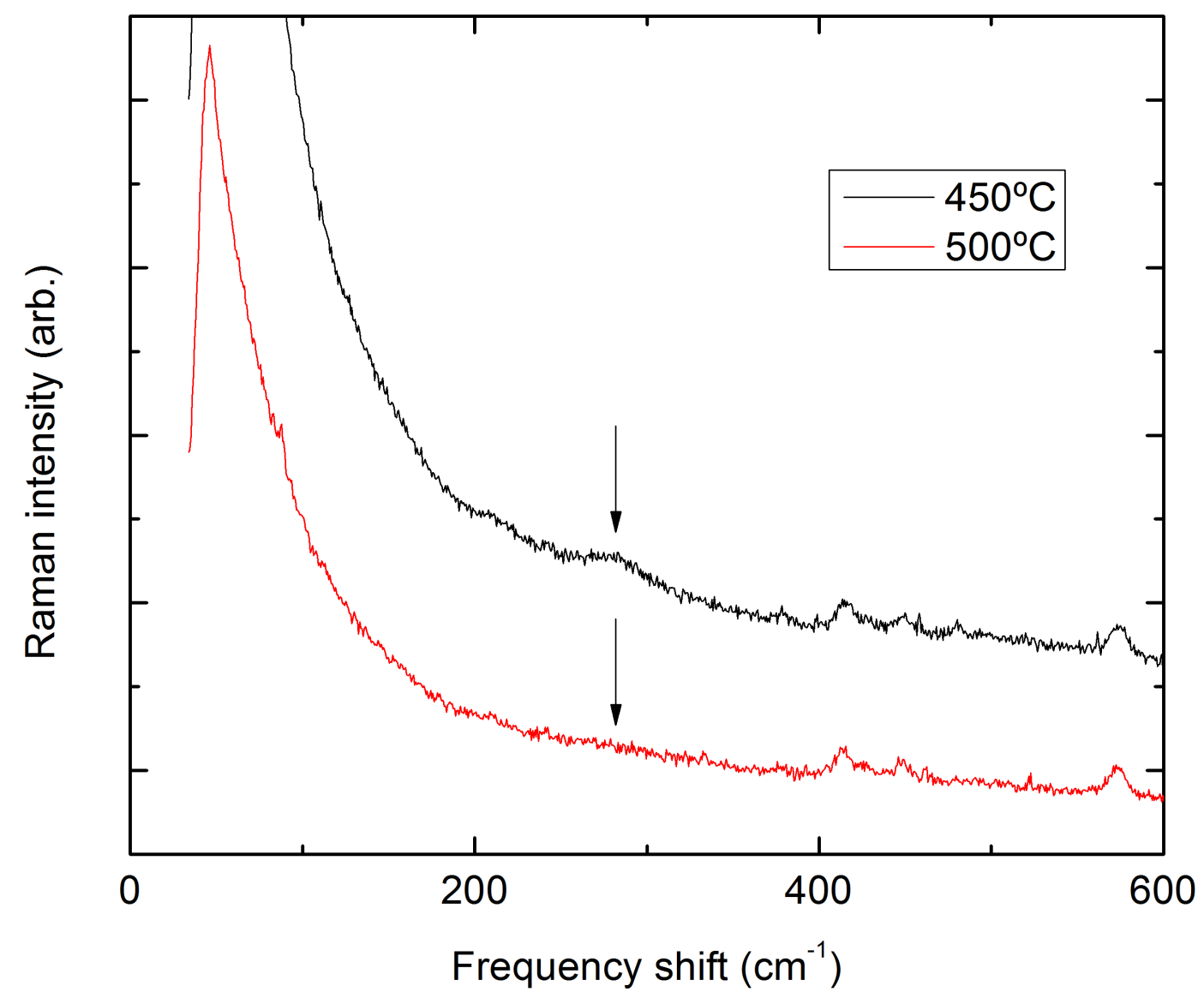

Figure 5.9: Between $450{ }^{\circ} \mathrm{C}$ and $500{ }^{\circ} \mathrm{C}$, the spectral lines from the PT disappear, suggesting a tetragonal to cubic phase transition. The spectra have been vertically offset for ease of viewing. This temperature is not elevated as are the other nanowire $\mathrm{T}_{C} \mathrm{~s}$ studies in this work. 
these spectra, from about $96 \mathrm{~cm}^{-1}$ at $200{ }^{\circ} \mathrm{C}$ down to about $74 \mathrm{~cm}^{-1}$ at $450{ }^{\circ} \mathrm{C}$. At $500^{\circ} \mathrm{C}$, we have a confirmation that the PT modes are gone - or at least that they are much much weaker. One could make an argument that there is still a peak at about $75 \mathrm{~cm}^{-1}$, but for sure the mode at $285 \mathrm{~cm}^{-1}$ is gone, and this has been a sign of a phase transition in the other wires. The spectra suggest that in these sub $100 \mathrm{~nm}$ wires, a phase transition occurs between $450{ }^{\circ} \mathrm{C}-500^{\circ} \mathrm{C}$, so in these wires there does not appear to be an enhancement of the phase transition temperature. It may be that the transition temperature is lower than in the bulk. This contrasts with the small other nanowires studied, which showed enhancement.

It is interesting that the frequency of the soft mode is higher in this sample than in the other wires measured, both far from the transition and near to it. It is possible that this is an artefact of the Lorentzian line subtraction, but could equally be a real effect - after all, a small decrease in the frequency was seen in the $125 \mathrm{~nm}$ wire which had the largest enhancement. Fig. 5.11 shows the frequency of the soft mode in this collection of sub $100 \mathrm{~nm}$ wires as a function of temperature in the spectra. The data from the $125 \mathrm{~nm}$ and $250 \mathrm{~nm}$ wires is provided for comparison. It's far from a comprehensive study, there being only four relevant points, but the fitting to a square root function, as was done for the other wires suggests a transition temperature closer to $450{ }^{\circ} \mathrm{C}$ than $500^{\circ} \mathrm{C}$, and it also suggests that the final softened frequency is closer to $75 \mathrm{~cm}^{-1}$ than to $50 \mathrm{~cm}^{-1}$.

\subsubsection{Final SEM and morphology change}

Subsequent to the Raman measurements, the samples were coated for SEM measurement, and high resolution SEM images were taken. However, when we came to view the wires again, there was a problem. Fig. 5.12 shows the region of PT nanowires, before and after the Raman measurements. It can be seen that after Raman measurements were taken, the PT is no longer in nanowire morphology, but has changed into lumps. At first we thought that the laser had been the cause of this morphology change. But we looked at other regions of the substrate, and found more places where small wire regions had changed into lumps, where the laser had not been applied. We therefore ruled out the influence of the laser in the change of morphology. Raised temperature is the next best candidate for causing 


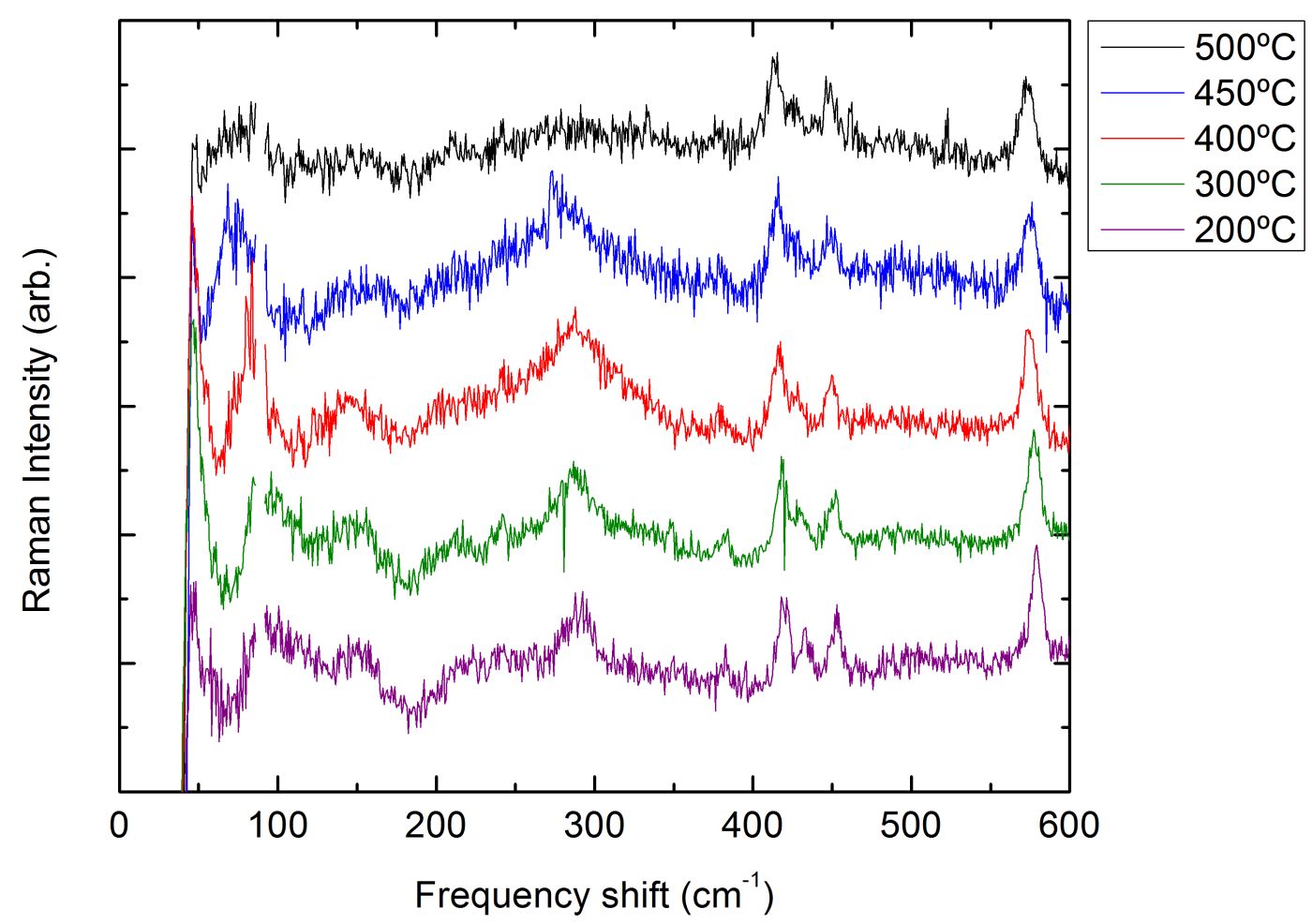

Figure 5.10: Temperature dependent Raman spectra of the region of sub $100 \mathrm{~nm}$ PT nanowires. The spectra have been vertically offset for ease of viewing. These spectra were produced by fitting a Lorentzian line to the central frequency noise and subtracting it from the spectrum. In these spectra the PT lines can be clearly seen, and the frequencies measured. Note the softening of the ferroelectric soft mode below $100 \mathrm{~cm}^{-1}$. The top line (black) is of the PT wires at $500^{\circ} \mathrm{C}$, and the disappearance of the PT lines can be clearly seen. 


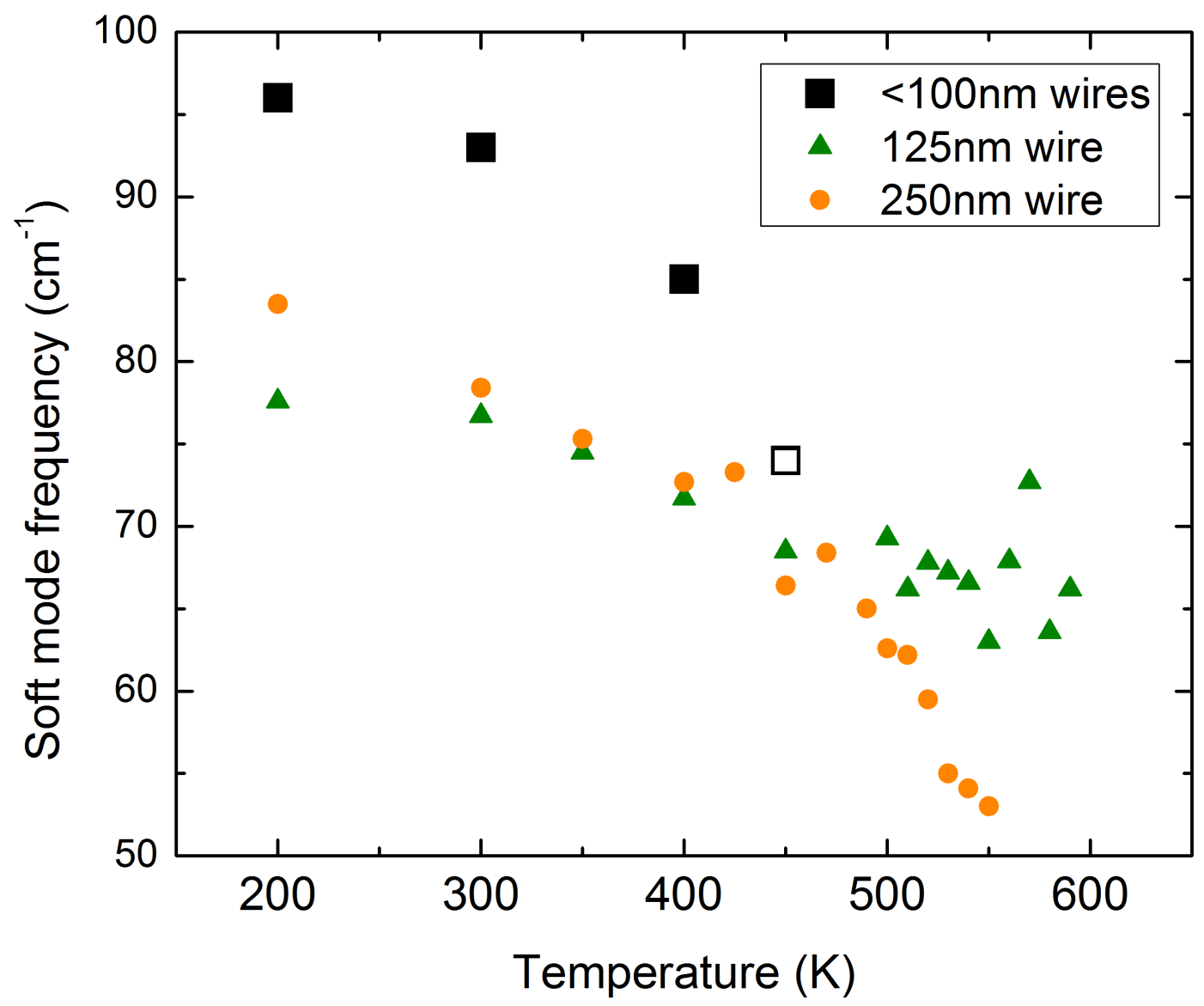

Figure 5.11: Temperature dependence of the ferroelectric soft mode in sub $100 \mathrm{~nm}$ PT wires (black squares) compared with $125 \mathrm{~nm}$ and $250 \mathrm{~nm}$ wires. The open symbol indicates that we cannot be certain whether this spectrum was affected by the morphology change or not. For the filled symbols, we can be confident that they were not. If the spectrum at $450 \mathrm{~K}$ is trusted, then this strongly indicates a comparatively lower $\mathrm{T}_{C}$ and the elevated frequency. If not, then the evidence for a lower $\mathrm{T}_{C}$ is not so clear. 
this change - it was a parameter significantly altered during the Raman experiments. However, the idea that high temperature could have caused the change in morphology is difficult to reconcile with the fact that the wires were fabricated in an annealing process in air at $540{ }^{\circ} \mathrm{C}$ - the experiment only took the samples up to $500^{\circ} \mathrm{C}$. However, during the annealing process, the wires were not diluted across a substrate. The increased surface area in the experimental arrangement compared with that of fabrication could have something to do with it. My best guess is that the increased surface area significantly impacted the equilibrium of the reaction transforming the morphology from sub $100 \mathrm{~nm}$ wires to lumps. During the fabrication, the surface area available to the reaction was small, so it proceeded slowly. During the experiment, surface area was increased and so the reaction occurred rapidly. Also potentially possible is that the wires we see in the dispersed sample of sub $100 \mathrm{~nm}$ diameter were part of larger structures during the fabrication, which protected them from the reaction. The act of sonication to separate the wires may have broken them off from the structures, so that they were vulnerable to change in the sample we measured. Whatever the cause, it is clear that the interpretation of our Raman spectra must take this morphology change into account.

Figs. 5.14 and 5.15 are SEM images showing the morphology of the wires after the experiment. It appears that where sub $100 \mathrm{~nm}$ wires were nearby to larger wires, they have formed lumps on that surface in preference to the sapphire. It indicates some mobility over the substrate, since in the sub $100 \mathrm{~nm}$ wire morphology they were not already in contact in all cases. In the region examined by Raman spectroscopy (refer figure 5.12, there was no nearby mesoscopic PT object, and the lumps have formed directly on the surface. Also replicated in Fig. 5.16 is a larger image of the $125 \mathrm{~nm}$ wire on sample 1 used in the study. Although the resolution is lower, note the lumps visible on the substrate. These are of similar dimensions and morphology to the lumps found on the more disperse substrate. In this case the lumps appear not to have coalesced onto the larger wires. However, these wires were on sample 1, which was brought to higher temperatures and were kept there for longer periods than the $<100 \mathrm{~nm}$ wires on sample 2 , which could explain some differences in morphology. Note that SEM images of the wires in sample 1 were taken only after cycling to high temperature. No wires of under $100 \mathrm{~nm}$ could be found in sample 1 . We had assumed that the lower concentration used in sample 2 was responsible for the finding of multiple sub $100 \mathrm{~nm}$ wires. But 


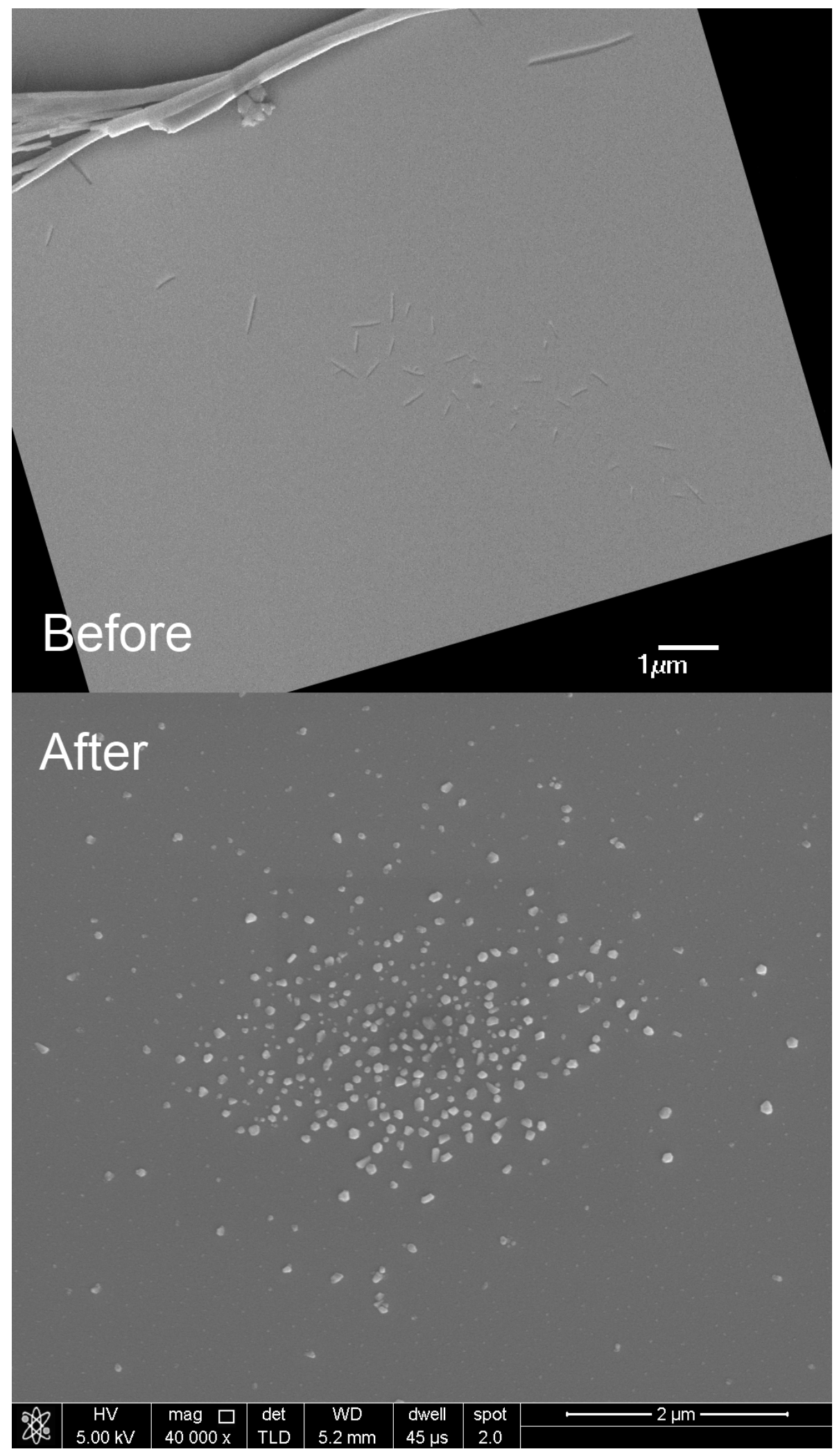

Figure 5.12: SEM images of Sub $100 \mathrm{~nm}$ PT nanowires before and after Raman measurement. The before picture has been rotated such that the substrate has the same alignment in both images. The wires have transformed in lumps. 
this image suggests that perhaps there were indeed such smaller wires present in the first sample before we cycled the wires up to $>500{ }^{\circ} \mathrm{C}$ for several hours, or otherwise caused the change by our Raman experiments.

One further feature worth noting in Fig. 5.13 is that it appears that the lower of the two wires in the bundle is longer in the after picture than in the before picture. Judging by the similarity of the shape of the bundle in Fig. 5.14, it seems unlikely that the wires have moved around. I think a better explanation is that some of the PT in the sub $100 \mathrm{~nm}$ wires has started growing on the lower wire visible, extending it. Coherent growth on larger nanowires such as this could explain the lack of lumps visible on the nanowires images on sample 1.

The fact that the morphology of the nanowires changed at some point during the Raman measurements makes interpretation tricky. We only have a before and after picture, and we can't confidently say when during the process of measurement the change occurred (or even if there was a single point - the change could have been gradual). Several factors in the Raman spectra suggest that what we measured is different from bulk PT, but small lumps may well have a spectrum that is different from bulk PT as well as small wires. Spectra were taken while temperature was being raised to $500^{\circ} \mathrm{C}$, so lower temperature measurements were chronologically earlier in the data shown so far. Fig. 5.17 shows subtraction spectra of the sample at $400^{\circ} \mathrm{C}$ after cycling up to $500^{\circ} \mathrm{C}$, and compares it to the spectrum at $400^{\circ} \mathrm{C}$ during the rise in temperature. In the after spectrum, the frequency of the soft mode is significantly lowered compared with the before spectrum - to 78 from $85 \mathrm{~cm}^{-1}$. This suggests that the morphology change occurred after $400{ }^{\circ} \mathrm{C}$ in the experiment. The three points $\leq 400^{\circ} \mathrm{C}$ alone still indicate a phase transition temperature $\ll 600^{\circ} \mathrm{C}$, which is in support of the high res TEM data which found the increased c/a result around $110 \mathrm{~nm}$, falling off on either side.

Now convinced that at least the three points $\leq 400^{\circ} \mathrm{C}$ were indeed measuring the PT nanowires rather than lumps of changed morphology, I am confident that the Raman data for this wire shows a phase transition between $450{ }^{\circ} \mathrm{C}$ and $500{ }^{\circ} \mathrm{C}$.

The interpretation of the Raman data on all of the nanowires is therefore that the wires with diameters close to $100 \mathrm{~nm}$ show an increased tetragonal to cubic phase transition temperature. The enhancement is dependent on wire diameter, 


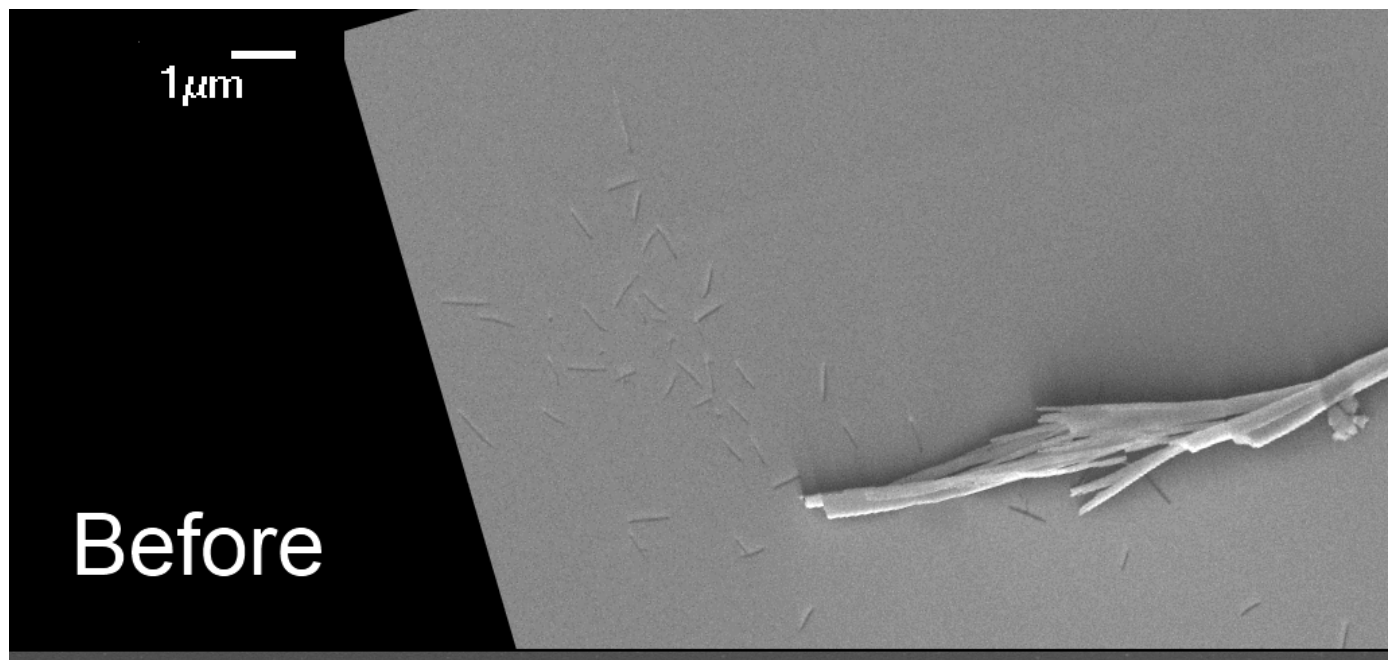

After

\begin{tabular}{|c|c|c|c|c|c|c|c|}
$*$ & HV & mag $\square$ & det & WD & dwell & spot & \\
\cline { 5 - 7 } & $5.00 \mathrm{kV}$ & $40000 \mathrm{x}$ & TLD & $5.2 \mathrm{~mm}$ & $20 \mu \mathrm{s}$ & 2.0 & \\
\hline
\end{tabular}

Figure 5.13: SEM images of Sub $100 \mathrm{~nm}$ PT nanowires before and after Raman measurement. The before picture has been rotated such that the substrate has the same alignment in both images. Seen in this image is a region of nanowires that was not within the Raman laser spot. It can be seen that the nanowires have also disappeared from this region, ruling out the influence of the laser in the morphology change. 


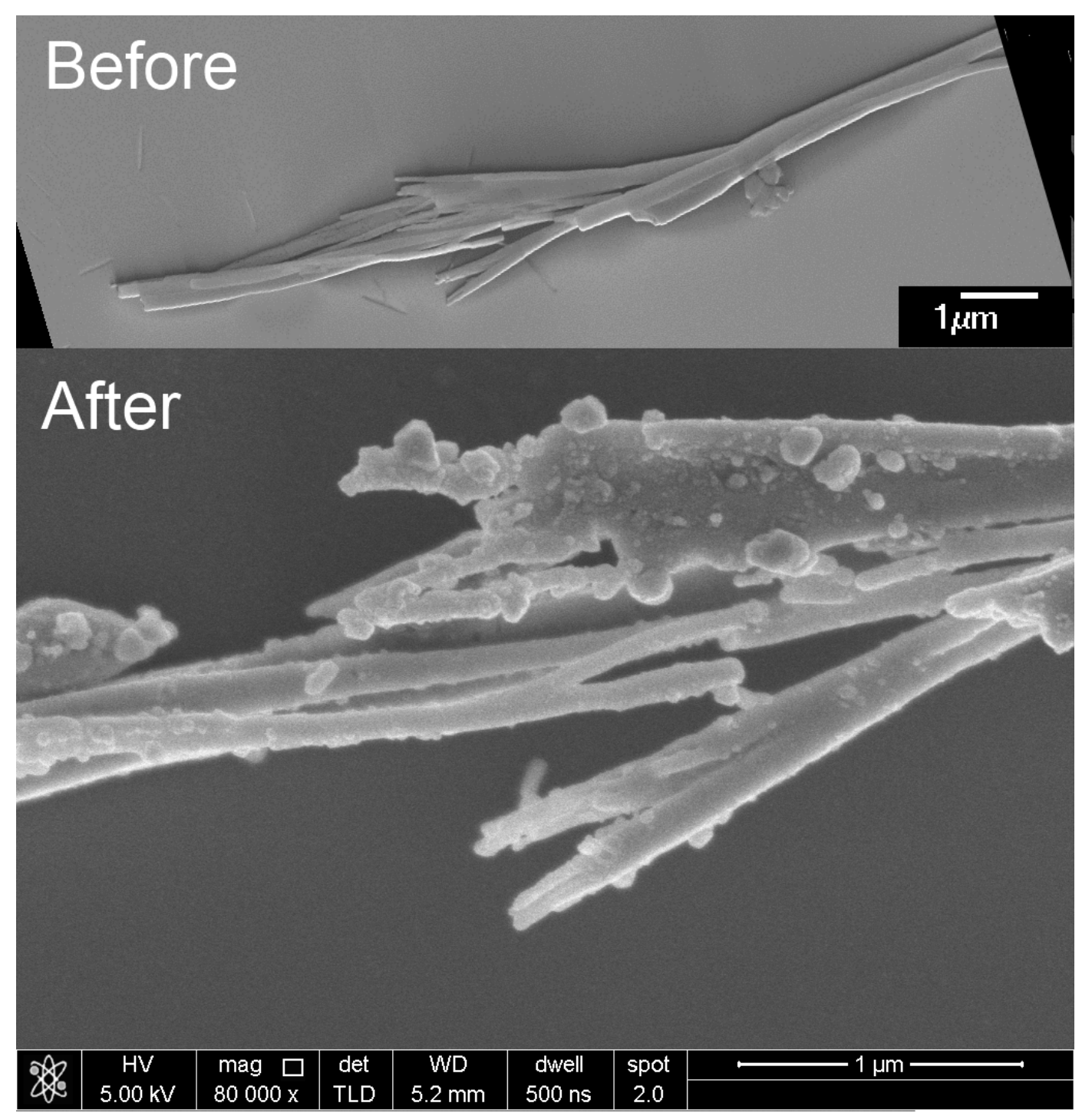

Figure 5.14: SEM images of Sub $100 \mathrm{~nm}$ PT nanowires before and after Raman measurement. The before picture has been rotated such that the substrate has the same alignment in both images. In this image we can see strong evidence of migration of the smaller wires into clumps on the surface of larger ones. Note also though, the vertically aligned wire in the middle of the after image. Although reduced in length, this particular smaller wire has retained its wire morphology. 


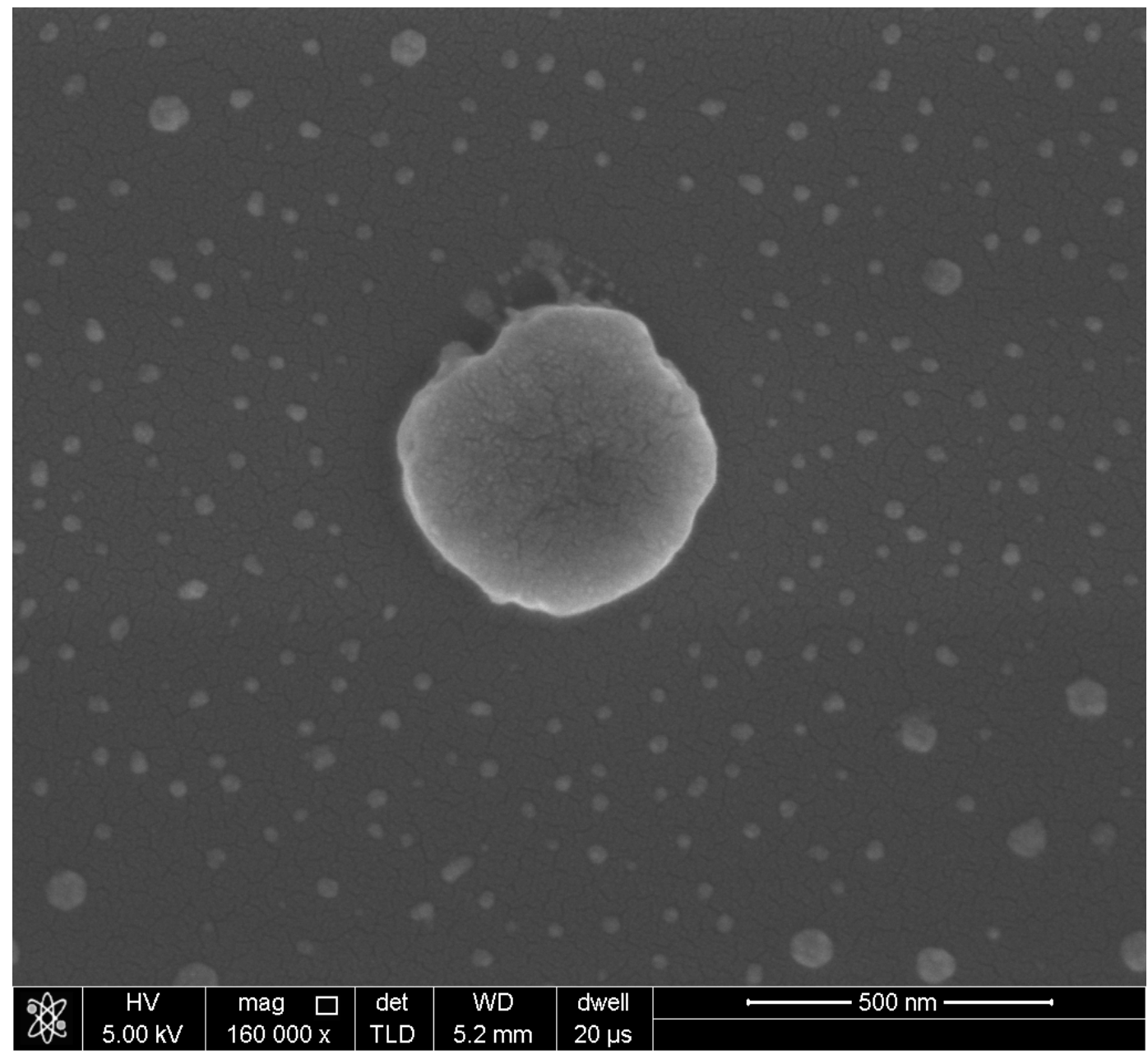

Figure 5.15: SEM images of Sub $100 \mathrm{~nm}$ PT nanowires after Raman measurement. Another image taken far from the site of Raman spectroscopy showing changed morphology of the PT nanowires. In this case, there was not significant migration of lumps onto the larger PT structure shown. 


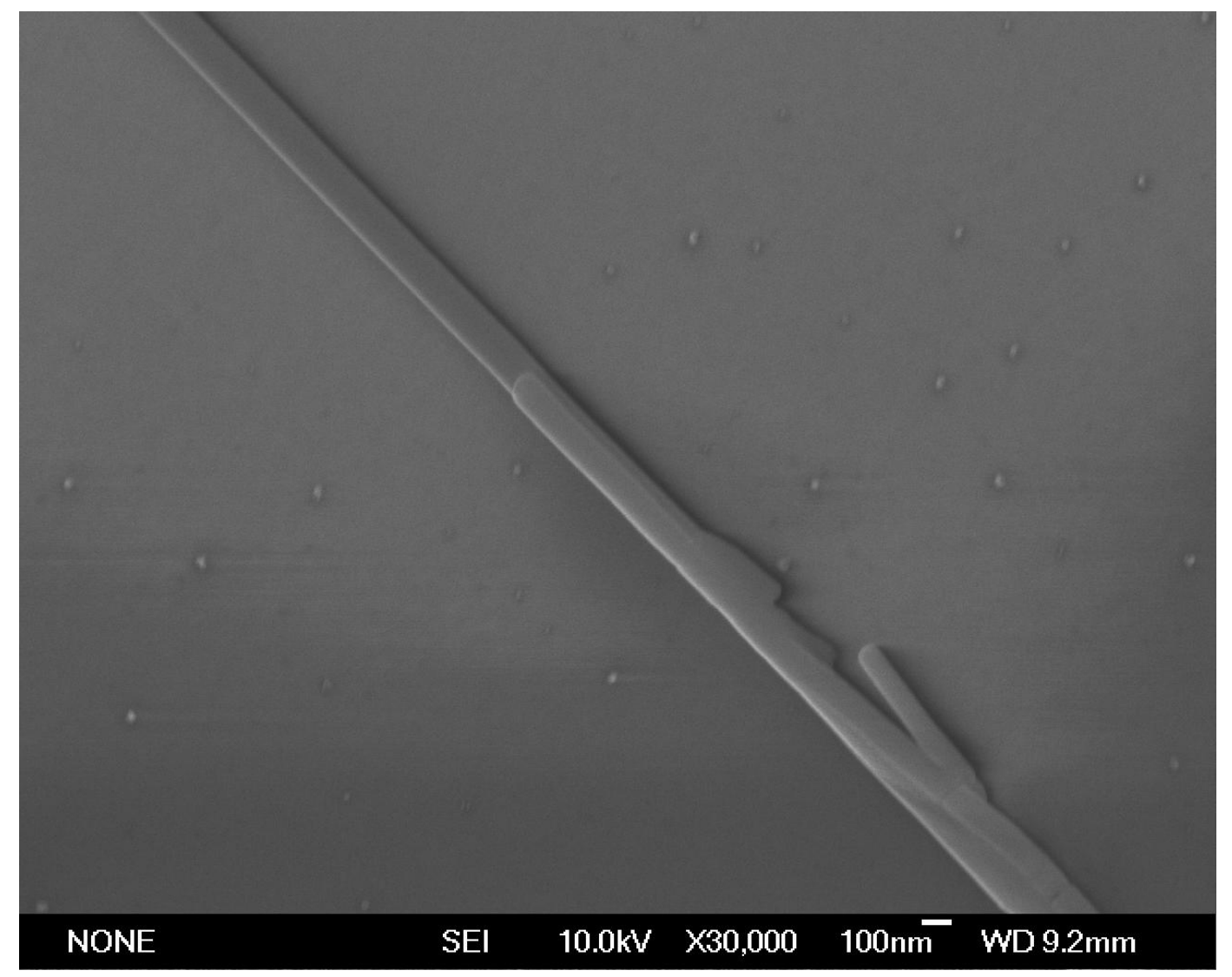

Figure 5.16: SEM image of $125 \mathrm{~nm}$ PT nanowire measured in this study. Note the small lumps visible on the substrate near the wire. Although smaller than those in the sub $100 \mathrm{~nm}$ PT nanowires sample, they could be a sign that there were once smaller PT wires on this substrate. 
rising as the wire diameter falls from $250 \mathrm{~nm}$ down to $125 \mathrm{~nm}$, and peaking at over $100 \mathrm{~K}$ enhancement, but falling back to the bulk value (or possibly slightly below) by $\sim 60 \mathrm{~nm}$.

\subsection{Discussion of enhanced $\mathrm{T}_{C}$}

What can explain the enormously enhanced phase transition temperature measured by Raman spectroscopy in these wires? It is implausible that small levels of impurities such as might be formed in the synthesis of these wires by annealing the hydrothermally created PX wires could raise the transition temperature by such a large degree, and that would not explain the wire diameter dependence of the enhancement.

High resolution TEM images have been taken of these wires by our collaborators Jin Wang et al. (these measurements not my contribution), and they give us a hint. Two other quantities were also found to be enhanced in these nanowires - the tetragonality (the ratio of the $c$ and $a$ crystal lattice constants), and the concentration of nanoscopic pores discovered in these wires. Fig. 5.18 shows TEM images of three wires of different diameters, and a plot of tetragonality vs wire diameter measured by TEM. The enhancement of the tetragonality was highly dependent on wire diameter, and peaked around $115 \mathrm{~nm}$ with a maximum value of twice the tetragonality of bulk PT, a consistent behaviour with the enhanced phase transition temperature measured in the Raman spectra. A large number of small circular voids, about $10 \mathrm{~nm}$ across can be seen in the volume of these wires. Note the high concentration of pores in the $115 \mathrm{~nm}$ wire, the relative absence in the $35 \mathrm{~nm}$ wire, and the complete absence in the $20 \mathrm{~nm}$ wire. The wires show a correlation between the pore concentration and the enhanced order parameters. In wires thicker than those in which we find enhanced tetragonality and Curie temperature, images show that cracks begin to form. Both the voids in the wires, and the cracks forming suggest that the wires to be (or to have been) under tensile strain. The excess volume is taken up by the cracks. Given the wires were synthesised by going from a low density phase (the PX-phase wires) into a high density phase (perovskite PT), it is plausible that tensile strain would be developed in these wires. 


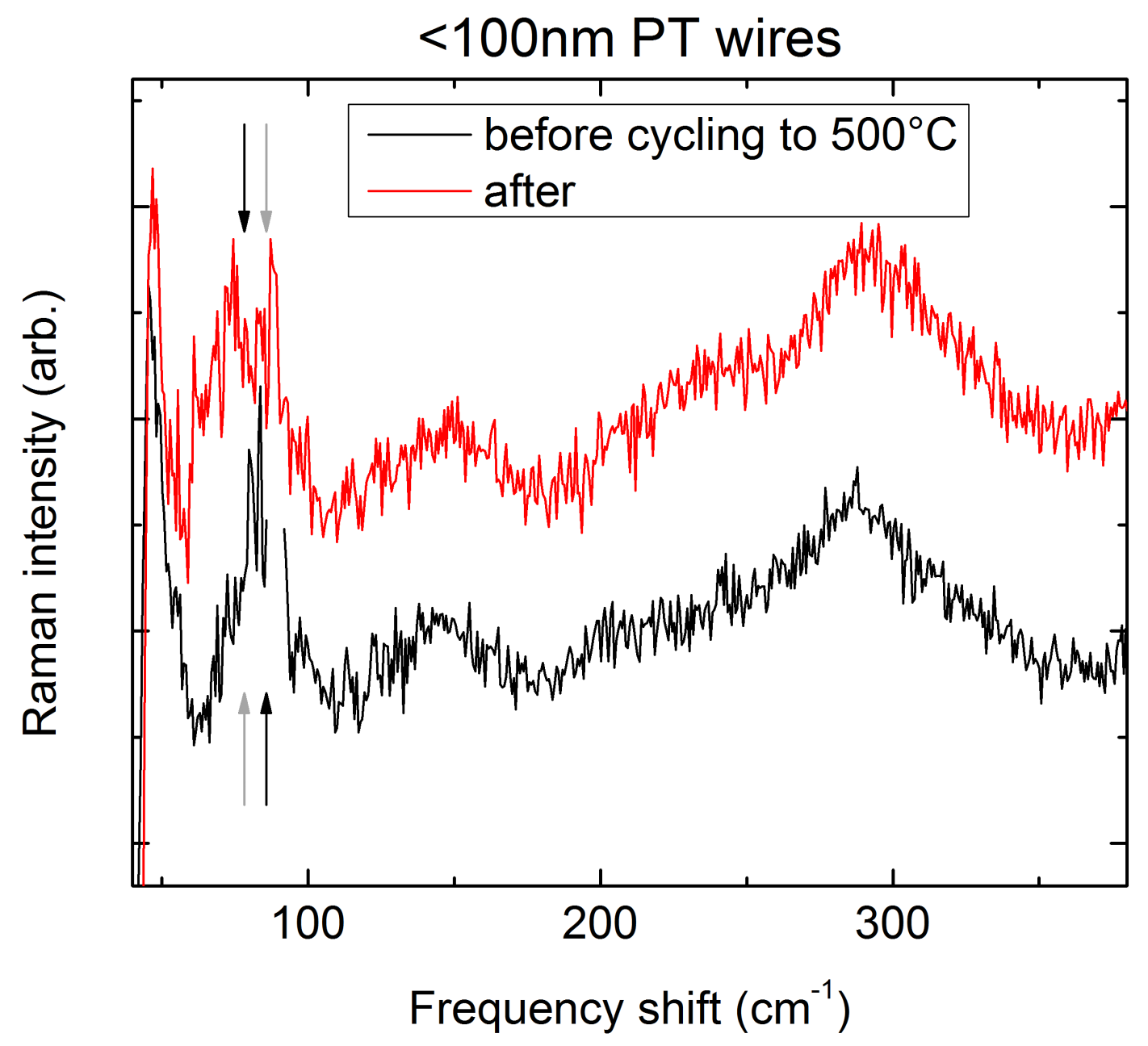

Figure 5.17: Raman spectra of sub $100 \mathrm{~nm}$ PT nanowires, both at $400{ }^{\circ} \mathrm{C}$, and both with a central frequency Lorentzian subtracted from the raw spectra. The spectra have been vertically offset for ease of viewing. The lower(black) spectrum was taken while temperature was rising, on the way up to $500{ }^{\circ} \mathrm{C}$. The upper (red) spectrum is the same sample taken on the way down, after the spectral change seen at $500{ }^{\circ} \mathrm{C}$. The position of the ferroelectric soft mode is noticeably different in the two spectra, while the other features are identical. 

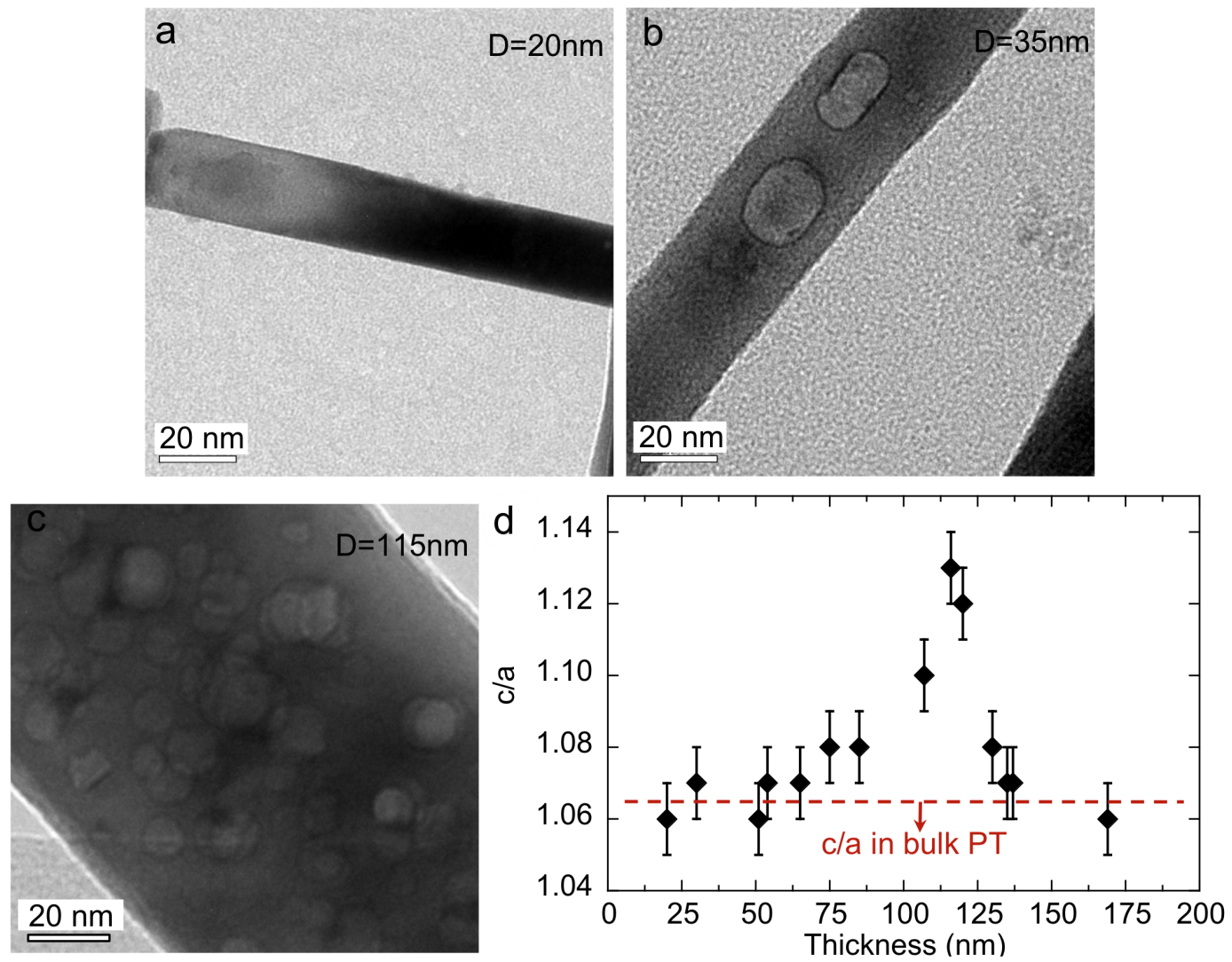

Figure 5.18: SEM images of three different PT nanowires of different diameters, and data for the tetragonality of these wires vs. wire diameter. The thinnest wire shows no circular voids. There are some voids in the middle wire, and many more in the thicker wire. The tetragonality of the wires is found to be enhanced. The enhancement is dependent on the diameter of the wires, with maximum enhancement of twice the bulk tetragonality found in wires of diameter of $115 \mathrm{~nm}$. 
More information and theory related to the proposal that tensile strain could be responsible for the enhancement in tetragonality and Curie temperature has been writen up into a paper under submission. A copy of the paper may be found in Appendix A.

Systems can easily be studied experimentally in monoaxial tensile strain (pulling apart) and biaxial strain (thin films with smaller natural lattice constants than their substrates) but if our explanation is correct, then this system is unusual for being under a significant tensile strain that is approximately hydrostatic (the strain is up to the order of GPa). Hydrostatic tensile strain approximates negative pressure, and is thus an exciting avenue for further study - quite apart from the obvious technical applications of higher Curie temperatures in ferroelectrics. If it can be replicated in other material systems, then it is a very feasible way of experimentally accessing materials under negative pressure, an exciting prospect that opens up many possible investigaitons.

\subsection{Conclusions - PT nanowires}

PT nanowires prepared by converting PX-phase PT nanowires to standard perovskite phase were measured by Raman spectroscopy, and by SEM imaging. Raman spectra showed an enhancement of the tetragonal to cubic structural phase transition in wires of diameters near to $110 \mathrm{~nm}$. The degree of enhancement was dependendent on nanowire diameter, and peaked between $60 \mathrm{~nm}$ and $125 \mathrm{~nm}$, with a value of greater than $100 \mathrm{~K}$.

TEM images were taken, and revealed the presence of small circular voids inside the wires. The images also showed an enhancement of the tetragonality in the wires, which showed a dependence on the nanowire diameter. The peak enhancement value was found at $110 \mathrm{~nm}$, and was twice as large as the tetragonality of bulk PT.

We propose that the cause of both the increased transition temperature and the increased tetragonality in the PT nanowires is hydrostatic tensile strain in the wires caused by the voids inside the material. This is the first report of a real 
system under significant negative hydrostatic pressure to the author's knowledge. 


\section{Chapter 6}

\section{Results - STO thin film system}

This chapter reports the results of Raman and XRD measurements of strained STO thin films. For this material, we had several samples all in different conditions of strain. We wanted to establish experimentally a relationship between the strain and the structural phase transition temperatures. There have been several theoretical works on the effect of biaxial strain in STO[1][24], and we felt that Raman studies would be a valuable contribution. A large part of this chapter is devoted to the interpretation of the Raman features in our samples. The features need to be isolated from those of the substrate, and then compared to the large body of literature in order to make assessments of the phase transition in this material. The assignment of the Raman spectra thus occupies a large amount of this chapter. A list of samples used in this chapter may be found in section 3.1.3, and the experimental method used in section 3.2.3.

\subsection{STO on STO}

Before we examine the strained thin film samples studied in this work, the results for STO grown epitaxially on an STO substrate will be shown. In this way, we can isolate the features that are related to strain. 


\subsubsection{Sample STO003 - STO/STO.}

Fig. 6.1 shows temperature dependent spectra of this sample, an STO thin film grown directly onto [001] STO substrate. The features present at room temperature are the well established second order STO features, and these show the usual temperature dependence: the $80 \mathrm{~cm}^{-1}$ peak is a difference mode ${ }^{1}$, and disappears to low temperature, the $300 \mathrm{~cm}^{-1}$ band is a combination of many modes, the shape changes as the difference modes leave the spectra to low temperatures, and the $800 \mathrm{~cm}^{-1}$ band is not significantly changed. Below $100 \mathrm{~K}$, a peak appears at $42 \mathrm{~cm}^{-1}$. This could be the AFD $\mathrm{A}_{1 g}$ soft mode, but it could also be an artefact of the stray light rejection, because this feature is right at the lower limit of the detection window. There is a band at $190 \mathrm{~cm}^{-1}$ at room temperature (denoted with an arrow), which softens significantly with lowering temperature. It becomes lost in the $42 \mathrm{~cm}^{-1}$ feature below $40 \mathrm{~K}$. This peak matches reasonably well with the $2 \mathrm{TO}_{1}$ feature seen in Nilsen and Skinner, which of course displays the same softening. Below $20 \mathrm{~K}$, there is significant increase in low frequency scattering, the central frequency below the spectral window measured. An increase in scattering of this kind is seen in Nilsen and Skinner. Finally, below $40 \mathrm{~K}$ there is a broad peak that appears at $165 \mathrm{~cm}^{-1}$. This peak does not have a similar feature in the Nilsen and Skinner spectra that I can relate it to. One does expect to see an AFD hard mode at about $150 \mathrm{~cm}^{-1}$, but that mode is very narrow, and the peak in our spectrum is broad. Instrumental broadening of this hard mode could not explain the width of the peak in the spectrum. Also absent from the spectra of the STO/STO film is the $450 \mathrm{~cm}^{-1}$ AFD hard mode, expected to appear concurrently with the $150 \mathrm{~cm}^{-1}$ AFD mode. The lack of the AFD modes could be explained by the weakness of the modes in the Raman spectrum. It is also possible that over the short length scales of the thin film, the AFD modes, which are activated by a doubling of the unit cell, are repressed. Certainly, there is a distinct lack of these peaks in the literature on Raman spectra of thin STO films. Drawing from that literature (in particular case study 5) the lack of these modes in our spectra may imply some strontium deficiency in our films.

\footnotetext{
${ }^{1} \mathrm{~A}$ difference mode is a second order mode involving the destruction of an excited phonon. As temperature tends towards zero, so does occupancy of excited phonon modes, and thus the intensity of difference modes tends towards zero.
} 


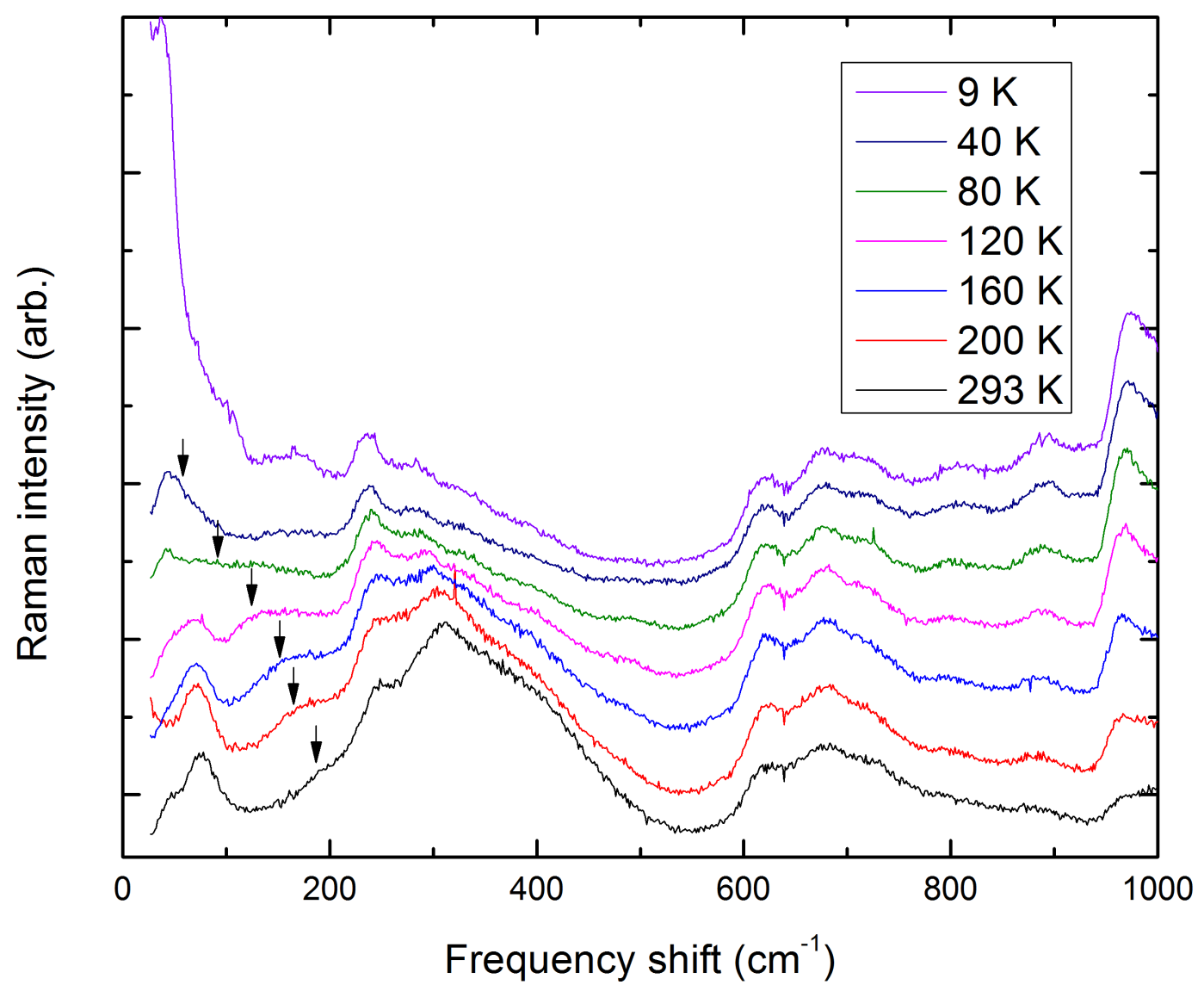

Figure 6.1: Temperature dependent Raman spectra of STO/STO thin film. The spectra are vertically offset for ease of viewing. The spectra match very well those of single crystal STO samples, with mostly second order features visible. The TO and LO modes are absent. The arrows mark out the ferroelectric soft mode scattering, similar to [27] and thought to be caused by polar microregions. 


\subsection{STO on LAO}

The samples in this section were grown on $\mathrm{LaAlO}_{3}[001]$, lattice mismatch of $-3.06 \%$ with STO (compressive)

\subsubsection{Sample 267, $100 \mathrm{~nm}$ STO/LAO: spectra in helium cryostat}

Spectra and assignment of modes

Fig. 6.2 shows the spectra of the STO thin film, and of the LAO substrate for comparison, both taken near room temperature inside the helium cryostat. We can immediately identify the strong, narrow peaks at $130 \mathrm{~cm}^{-1}, 153 \mathrm{~cm}^{-1}$, and $488 \mathrm{~cm}^{-1}$ as belonging to the substrate. Two more peaks - at $103 \mathrm{~cm}^{-1}$ and $204 \mathrm{~cm}^{-1}$ - are seen in the substrate, but are not visible in the thin film, likely due to the weakness of these peaks compared to the other substrate peaks. That leaves the broad features between 250 and $450 \mathrm{~cm}^{-1}$, and between 600 and $850 \mathrm{~cm}^{-1}$ as being assignable to the STO thin film. These match well with the expected second order features of STO. Note though that the other second order feature, a band at $80 \mathrm{~cm}^{-1}$, is not visible in this spectrum. There is also a very strong peak in the STO spectrum at $34 \mathrm{~cm}^{-1}$. Due to its strength, this feature almost certainly is due to the substrate, but I was unable to resolve peaks in the substrate spectrum this low in frequency at room temperature due to a high level of low-frequency scattering.

Fig. 6.3 shows spectra of the same samples, this time measured at $9 \mathrm{~K}$ inside the helium cryostat. In the substrate the peaks at 130, 153, and 488 wavenumbers are still present, but the lowest peak has shifted from $130 \mathrm{~cm}^{-1}$ to $143 \mathrm{~cm}^{-1}$. In addition to these, there are new weak peaks in the substrate at $470 \mathrm{~cm}^{-1}$ and $709 \mathrm{~cm}^{-1}$, and we are able to resolve a strong peak at $34 \mathrm{~cm}^{-1}$ due to increased narrowness of peaks at low temperatures, and an absence of low-frequency scattering. This confirms the tentative assignment of the $34 \mathrm{~cm}^{-1}$ peak seen in the film spectrum at high temperature. The additional features visible in the STO film 


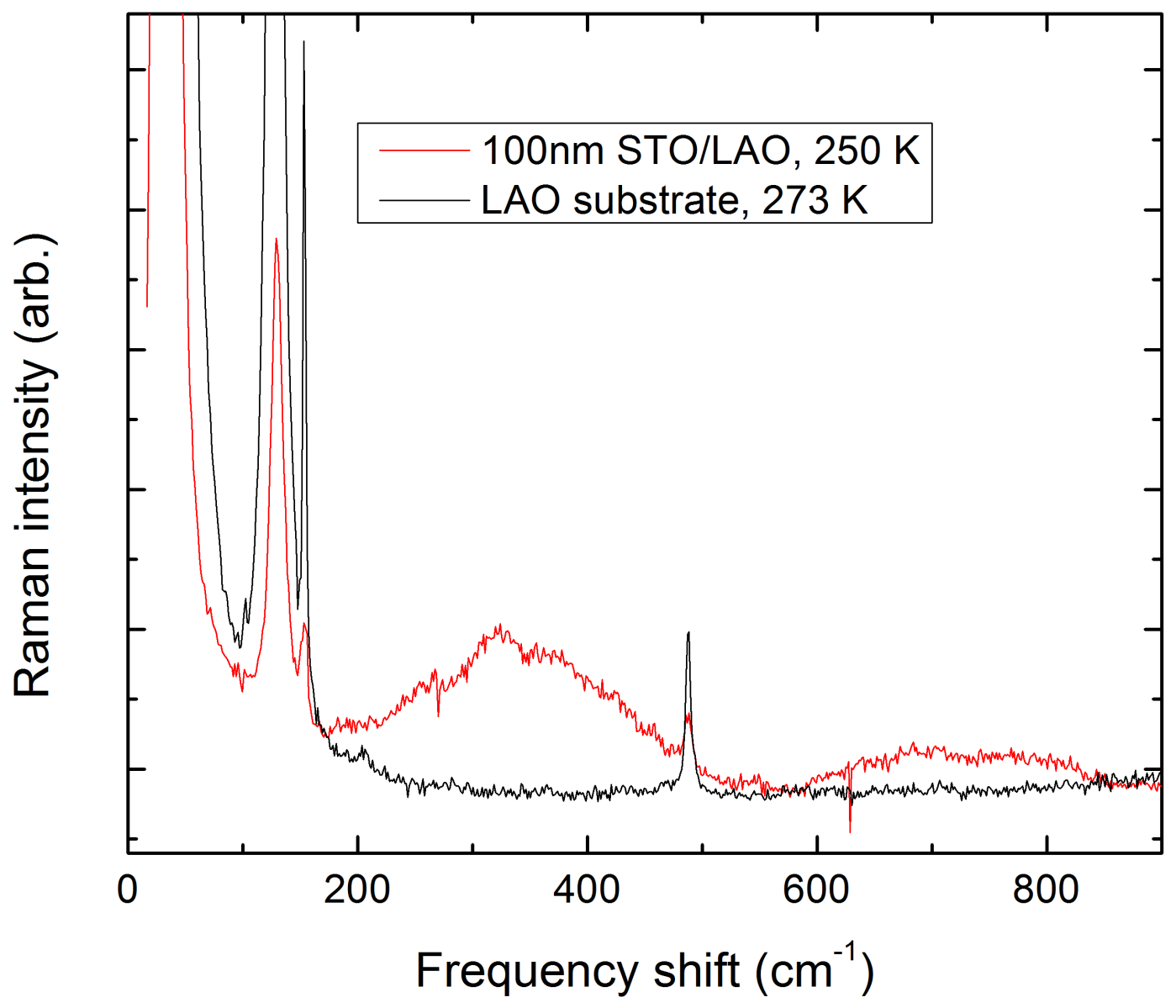

Figure 6.2: Raman spectra of sample STO267 (red), and the LAO substrate on which it is grown (black), both at room temperature. Substrate peaks and second order features from STO can be seen in the thin film. 


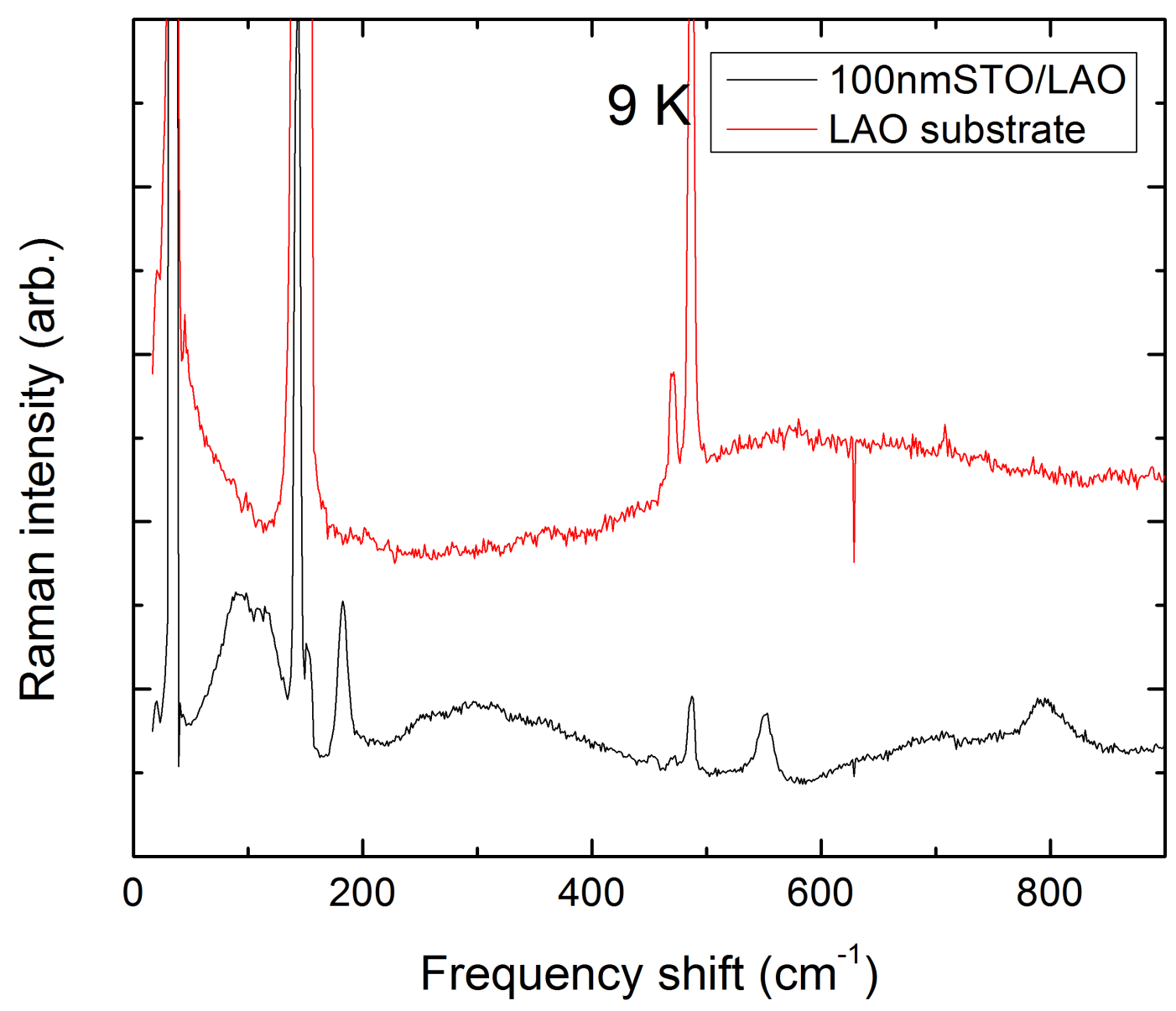

Figure 6.3: Raman spectra of STO267 and LAO taken at $9 \mathrm{~K}$. At this temperature, there are many more lines in the film spectrum than in the substrate.

spectrum are: broad features between $350-400 \mathrm{~cm}^{-1}$, and $600-800 \mathrm{~cm}^{-1}$, the second order scattering bands are still present and they show the alteration of shape expected in unstrained bulk STO. In addition, there are strong, narrow peaks at $183 \mathrm{~cm}^{-1}$ and $550 \mathrm{~cm}^{-1}$, a strong broad peak at $794 \mathrm{~cm}^{-1}$, a strong double peak feature at $93 / 112 \mathrm{~cm}^{-1}$, and a very weak peak at $452 \mathrm{~cm}^{-1}$.

Figs. 6.4 and 6.5 show spectra of this STO thin film taken at a range of temperatures in the helium cryostat, and show the temperature dependence of the STO modes. The spectra in these figures have been vertically offset for ease of viewing.

The modes at 180,550 , and $790 \mathrm{~cm}^{-1}$ all have the same temperature dependence. They are intense at low temperatures, and gradually weaken with rising 


\section{0nm STO/LAO film}

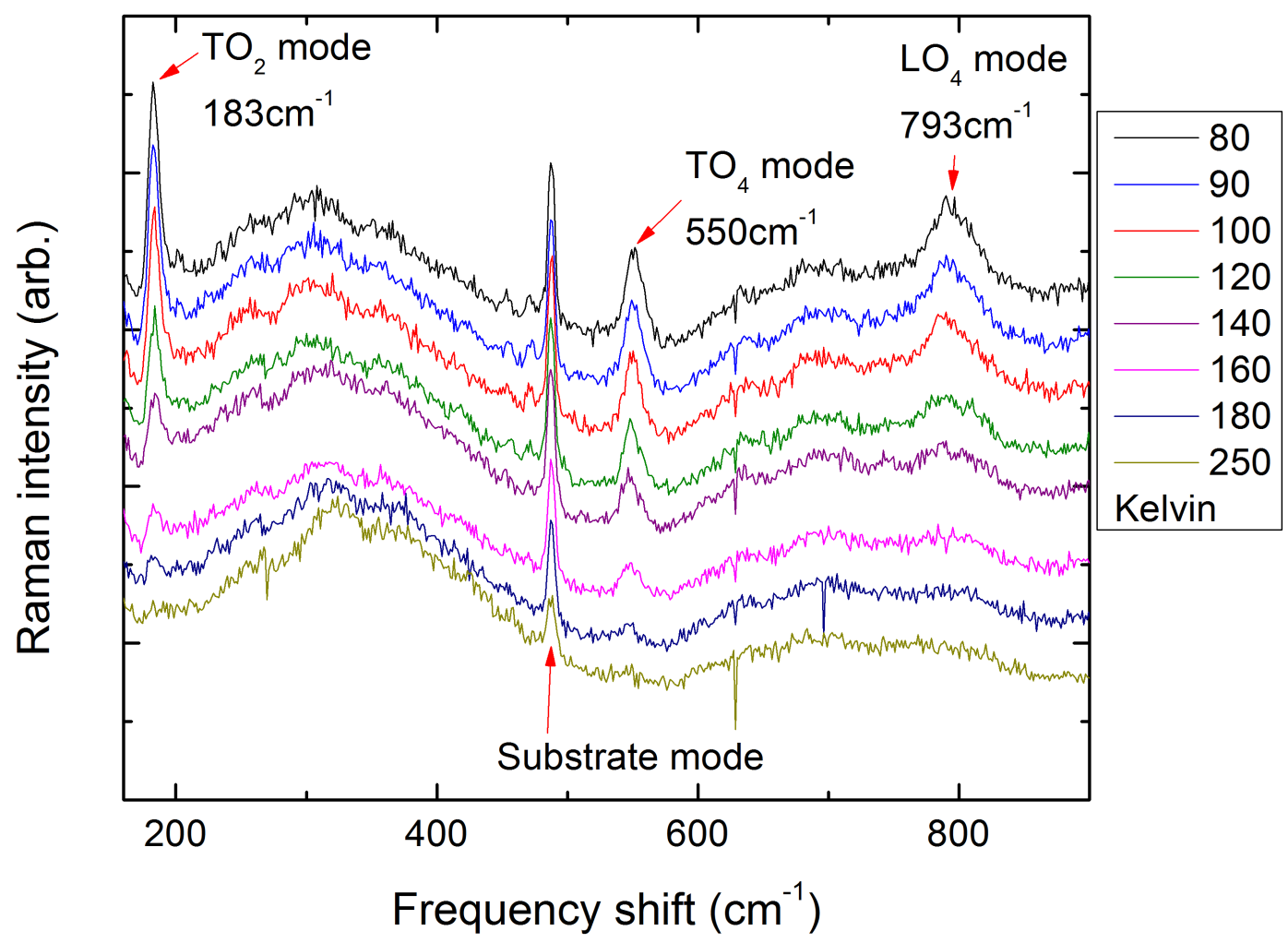

Figure 6.4: Temperature dependent Raman spectra of STO267 showing the emergence of the first order modes in STO. The spectra are vertically offset for ease of viewing.

temperature, eventually disappearing near $300 \mathrm{~K}$. The frequency of these modes does not change with temperature. Their positions and behaviour are a good match to the $\mathrm{TO}_{2}, \mathrm{TO}_{4}$ and $\mathrm{LO}_{4}$ modes commonly seen in the literature. The $\mathrm{TO}_{2}$ mode shows a Fano-type asymmetry, as is commonly seen of this mode in the literature. However, the degree of asymmetry is not nearly as high as in many samples - particularly ceramic and polycrystalline samples show a high degree of asymmetry. The $\mathrm{TO}_{2}$ peak in these spectra is comparatively quite symmetric. These polar hard modes have similar line widths to the $6 \%$ Ca-substituted STO sample in case study 1.

The mode at $450 \mathrm{~cm}^{-1}$ also displays no softening with temperature. Its amplitude is difficult to gauge because of its weakness, but it appears to stay constant with temperature between $9 \mathrm{~K}$ and $120 \mathrm{~K}$, and then drop suddenly to zero by $140 \mathrm{~K}$. 


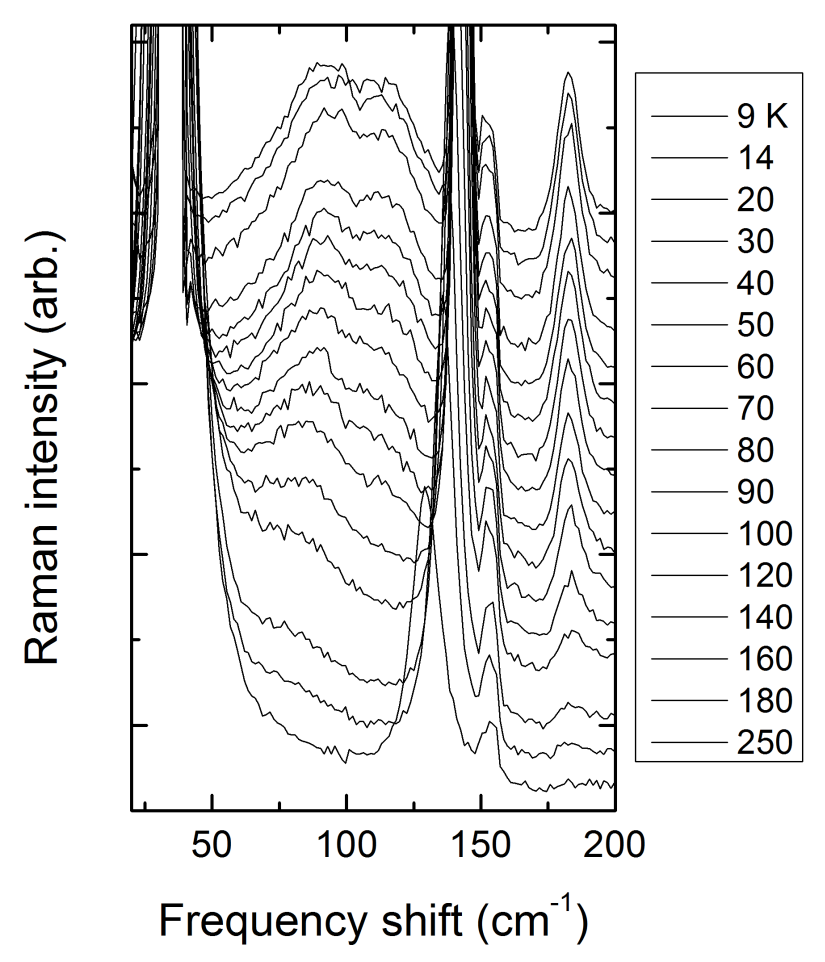

Figure 6.5: Temperature dependent Raman spectra of STO267, showing a close up on the $100 \mathrm{~cm}^{-1}$ feature. The spectra are vertically offset for ease of viewing.

It is possible that this temperature dependence is the same as that of the hard polar modes - The hard polar modes have dropped to half of their low temperature intensity by $120 \mathrm{~K}$, and half of the $450 \mathrm{~cm}^{-1}$ mode's $9 \mathrm{~K}$ intensity is smaller than the peak-to-trough noise intensity present in the spectra. This mode could possibly be an R-point hard mode associated with the AFD phase (refer to section 2.3.2). It appears at the right frequency for this, and the AFD modes are commonly observed to be weak or absent in thin films of STO. However, the AFD hard mode amplitudes usually display no temperature dependence after they have appeared, and for this sample XRD measurements have been made which show very convincing evidence of a cubic to AFD phase transition at $400 \mathrm{~K}$ (this XRD is not my contribution, it was performed by Tomoaki Yamada at Tokyo Institute of Technology). Fig 6.6 shows the XRD. One can see the emergence of a superlattice peak related to the AFD unit cell doubling. The temperature dependence of this mode in the Raman is therefore not well explained by this. Other AFD hard and soft modes are expected to be seen simultaneously with the one at $450 \mathrm{~cm}^{-1}$. However, due to the interference of substrate peaks, I cannot tell whether these peaks are present or not in this sample. Another possibility is that the peak is an- 


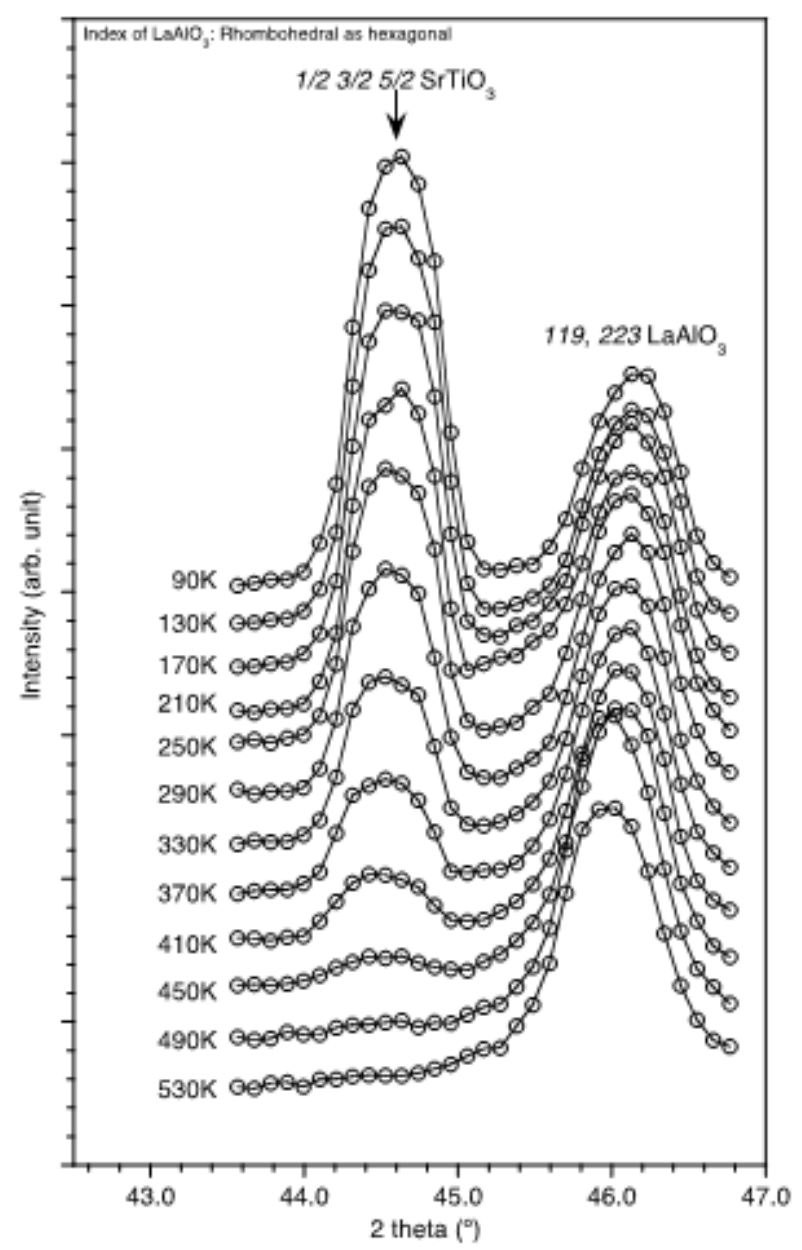

Figure 6.6: Temperature dependent XRD spectra showing the emergence of the characteristic AFD superlattice peak at about $400 \mathrm{~K}$. The spectra have been vertically offset for ease of viewing. This work was not my contribution.

other feature associated with polar microregions or the orthorhombic phase. The temperature dependence seems to match the polar hard mode dependence well, however there is no previous report of another mode at this frequency besides the AFD hard mode. I cannot make a confident assignment of this peak.

The peaks around $100 \mathrm{~cm}^{-1}$ at $9 \mathrm{~K}$ have an interesting temperature dependence - they show some variation in frequency as well as intensity. As the temperature rises, the lower frequency peak starts to soften, decreasing from $94 \mathrm{~cm}^{-1}$ to $76 \mathrm{~cm}^{-1}$ at $180 \mathrm{~K}$. By $200 \mathrm{~K}$ it has dropped to zero measurable intensity. The higher frequency peak does not change measurably in frequency, and its intensity drops until it is undetectable at $120 \mathrm{~K}$. Whether it is just lost in the other peak at this point, 
or if it has actually dropped towards zero is not certain, but for sure its intensity has dropped faster than the lower peak of this doublet feature.

\section{Analysis of Raman features}

The polar hard modes $\mathrm{TO}_{2}$ and $\mathrm{TO}_{4}$, and $\mathrm{LO}_{4}$ were fitted with Lorentzian peaks at each temperature using a least-squares fitting process. Fig. 6.7 shows temperature dependence of the height of the polar hard modes in this sample. The height parameter (the area of the peak divided by its width) was used as the fitting parameter rather than amplitude because of the interference to the fitting process of the substrate peaks. A rigorous subtraction of the substrate spectra was not easy to achieve due to differences in the relative weight of substrate features between the substrate and sample spectra, and other features such as low frequency noise. therefore I thought it best to fit the spectra together with the substrate peaks rather than to attempt a dubious subtraction that would introduce artefacts into the analysis. The scattering amplitude of the substrate peaks reduced the ability of the fitting software to converge to a single good fit, rather a range of shapes were able to reasonably fit the spectra, essentially assigning the amplitude between the STO peaks and substrate peaks in a number of different ways. However, the height parameter of the fits was greatly more consistent across this range of solutions, hence its use over amplitude in this analysis.

An S-shaped curve is seen in the temperature dependence, as is seen in other samples of STO in the literature. Note that each figure in the stack is scaled to display over the whole area, the height scales are not consistent between modes. The TO modes have very similar temperature dependence. The curve for the TO modes is approximately $100-150 \mathrm{~K}$ wide, achieving a saturation of intensity at around $30-40 \mathrm{~K}$. This width is significantly larger than the ferroelectric $0.7 \%$ Ca substituted STO single crystals, but significantly smaller than the non-ferroelectric, non-stoichiometric thin films. The most similar example is the $1.3 \mu \mathrm{m} \mathrm{STO/LAO}$ films studied by Sirenko et al. [52], although there is also a similarity with the more highly $(6 \%)$ Ca-substituted samples studied by Ouillon et al.[19]. The LO mode is more difficult to fit than the other modes, being broader and sitting on top of a second order feature. Nevertheless, we are still able to get an acceptable level of 
signal to make the fitting up to $180 \mathrm{~K}$. The $\mathrm{LO}_{4}$ mode shows a slightly different temperature dependence to the TO modes, in that it saturates at a higher temperature - $70-80 \mathrm{~K}$ rather than $30-40 \mathrm{~K}$. The sharpest decline in the S-shaped curve still occurs at roughly the same temperature as the TO modes, between 120 and $140 \mathrm{~K}$, and the high $\mathrm{T}$ data looks the same as far as we are able to conduct the fitting.

The $100 \mathrm{~cm}^{-1}$ feature I could not assign easily. From examination of the literature of Raman studies on STO, there are two leading candidates for explaining its presence in the spectra - the ferroelectric soft mode $\left(\mathrm{TO}_{1}\right)$ and the AFD soft modes in the tetragonal AFD phase.

As previously mentioned, many of the papers in literature have seen a peak near $100 \mathrm{~cm}^{-1}$ similar to the one seen in the spectra of this sample, and have assigned it to the ferroelectric soft mode $\left(\mathrm{TO}_{1}\right)$, on the basis of spectra of reduced STO showing higher frequency $\mathrm{TO}_{1}$ than normal. It is possible that this feature at $100 \mathrm{~cm}^{-1}$ could be the ferroelectric soft mode in sample STO267. In the literature, many effects have been shown to increase the frequency of the ferroelectric soft mode, sometimes as high as the $100 \mathrm{~cm}^{-1}$ seen in the feature here. The appearance and strengthening of this feature simultaneously to the other first-order modes would support this assignment. Fig 6.8 shows the variation in intensity of the lower frequency peak compared to the $\mathrm{TO}_{2}$ peak. It is clear that both modes show the same temperature dependence. If this is $\mathrm{TO}_{1}$, it seems unlikely that the mode softens to zero frequency, as is usual in a ferroelectric phase transition in STO. It softens towards higher temperatures, indicating a critical point at or above $200 \mathrm{~K}$. An incomplete softening to above zero and at an elevated temperature, similar to what is observed of this mode is seen in [44], and that sample is proven nonferroelectric by permittivity measurements. The non-ferroelectricity is a common feature to all samples reported to have the ferroelectric soft mode hardened as much as is shown in our sample. On the other hand, this peak is clearly a doublet, and appears to show a splitting at around $100 \mathrm{~K}$, and this is generally a strong sign of a phase transition - in the cubic and AFD modes of STO the ferroelectric soft mode is triply degenerate, splitting into different frequencies only in the ferroelectric, orthorhombic phase. So the implication of this feature is not clear.

It could also be possible that the peaks seen here are components of the AFD 


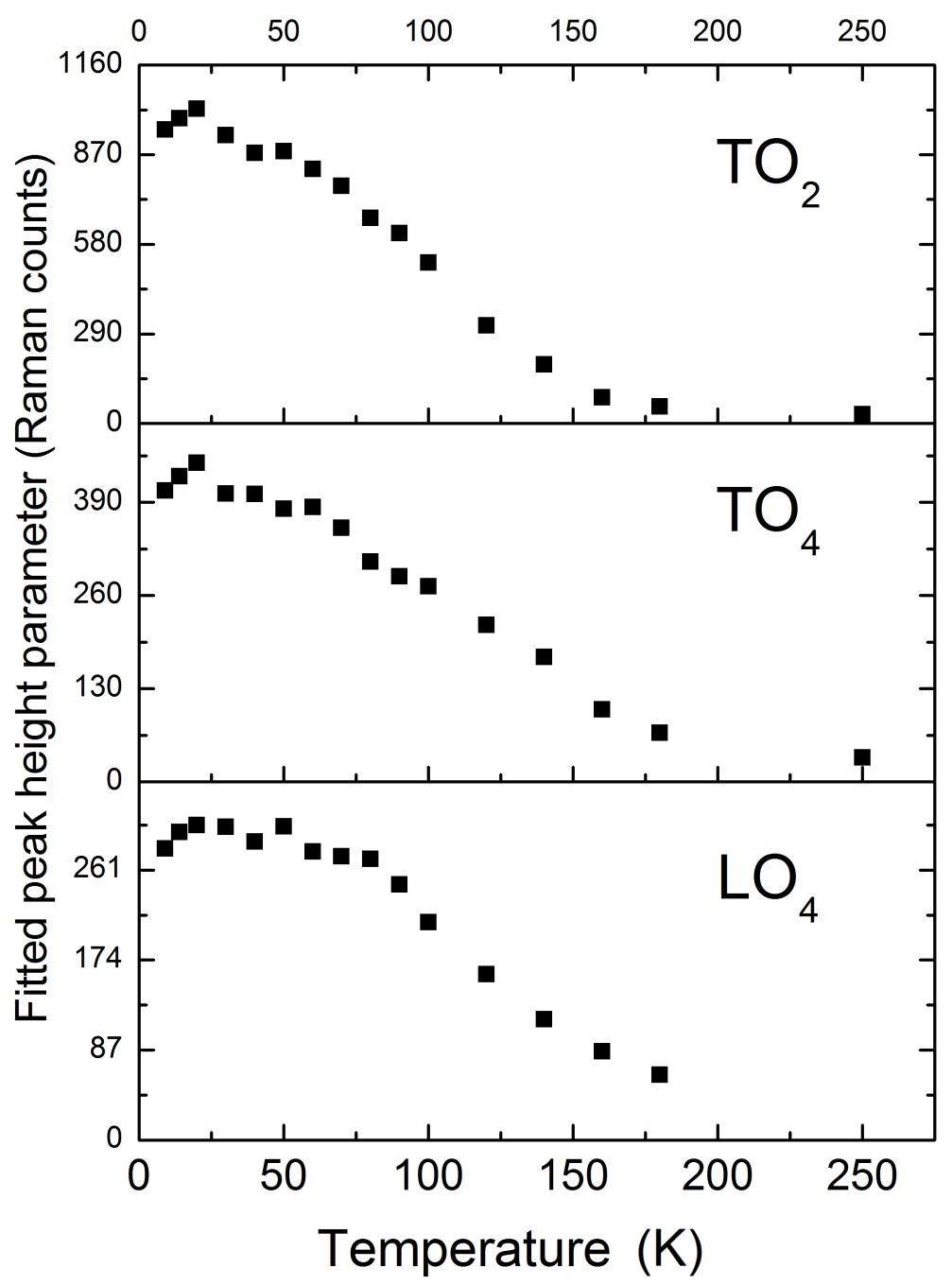

Figure 6.7: Temperature dependence of the three first-order mode peak heights visible in STO267. The vertical scales are not the same in each of the three plots. It can be seen that all three peaks intensities have the same temperature dependence. 
soft mode. These peaks harden only to $20 \mathrm{~cm}^{-1}$ and $50 \mathrm{~cm}^{-1}$ respectively in bulk unstrained STO, but given that we know the AFD phase transition to occur at a greatly elevated temperature in this sample compared to the bulk case, it could be possible that the frequency of the AFD modes changes too. The modes seen in the spectra of this sample are also much broader than the AFD modes where they are seen in the literature. The small film volume may contribute to a broadening of that peak, but one would not expect it to broaden them to to be as wide as the peaks in this sample. Finally, if the feature is indeed the AFD soft mode, then some other explanation needs to be given for their appearance in the spectra about $200 \mathrm{~K}$ below the point where the phase transition occurs.

Neither of those exaplanations adequately explains the frequency and the temperature dependence of these peaks in the Raman spectra. This feature, like the $450 \mathrm{~cm}^{-1}$ feature, I cannot confidently assign. I will come back to the subject of this feature in section 6.6 after having reported on the spectra from the other samples. As it turns out, this feature is present in many of the other samples and looking at all of them together can aid the analysis. Based on all of the data, I will propose that the most likely explanation for these peaks is the $\mathrm{TO}_{1}$ mode in regions of the film which are largely relaxed from the strain of the substrate.

In summary: this sample of STO clearly shows polar hard modes $\mathrm{TO}_{2}, \mathrm{TO}_{4}$ and $\mathrm{LO}_{4}$. There are two features, one at $100 \mathrm{~cm}^{-1}$ and one at $450 \mathrm{~cm}^{-1}$ that I could not confidently assign. The temperature behaviour of the polar hard mode intensities displays an S-shaped curve as seen elsewhere in the literature. The temperature range of this curve is about $160 \mathrm{~K}$, which is significantly more than in the most clear examples of ferroelectric STO in the literature.

\subsubsection{Sample STO267, $100 \mathrm{nmSTO/LAO:} \mathrm{spectra} \mathrm{in} \mathrm{nitrogen} \mathrm{cryostat}$}

Fig. 6.9 shows Raman spectra of the same sample (STO/LAO 100nm) taken in the nitrogen cryostat at $80 \mathrm{~K}$ compared with the same sample at $80 \mathrm{~K}$ in the helium cryostat. Note that the line for the helium cryostat measurement has been multiplied by a scale factor of 7 , in order to fit both lines comfortable on the graph. In the nitrogen cryostat configuration, a microscope objective is used for spot forming and collection, meaning that the depth of focus is far shorter than in 


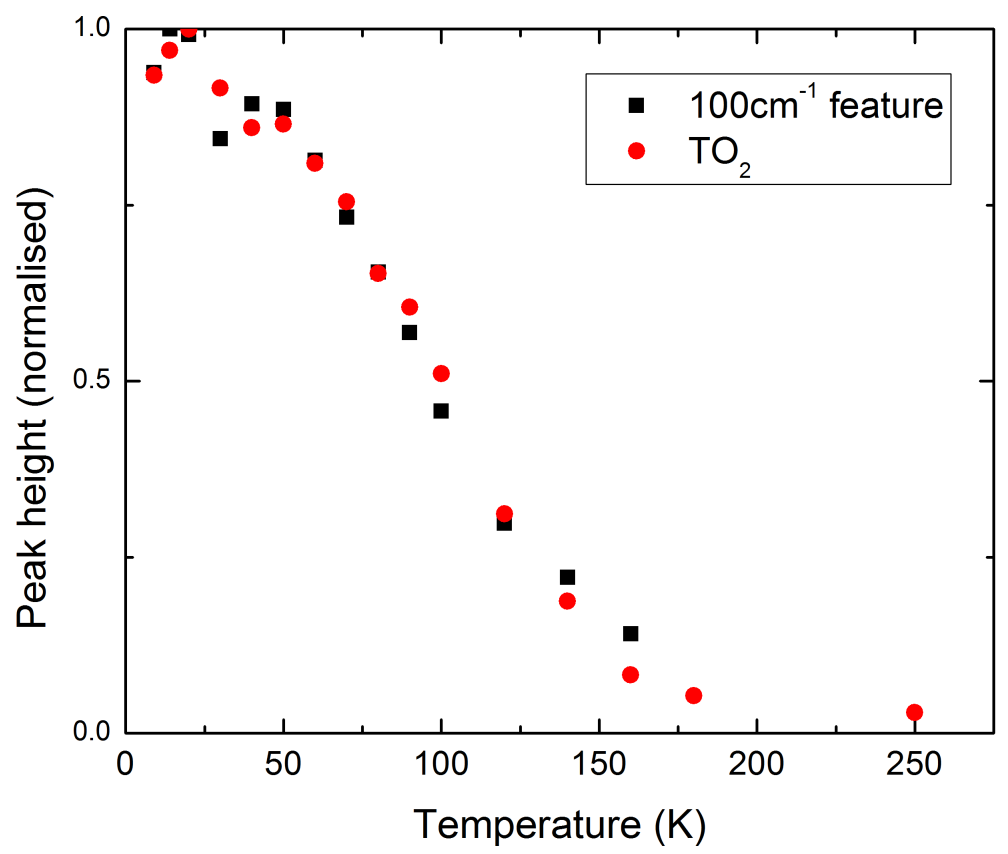

Figure 6.8: Temperature dependence of the $100 \mathrm{~cm}^{-1}$ and $\mathrm{TO}_{2}$ mode peak heights in sample STO267, taken from Lorentzian fits to the data. The intensities of the modes vary almost identically with temperature, which is a strong indication that the $100 \mathrm{~cm}^{-1}$ feature has the same origin as the other first order modes.

the helium cryostat. As a consequence, the surface sensitivity is enhanced, but in this configuration $77 \mathrm{~K}$ is the lowest accessible temperature. The biggest noticeable differences in the spectra are: First, the resolution is lesser in the nitrogen cryostat. This is not an inherent characteristic of the microscope configuration, the slits were intentionally opened wider for this series of measurements as high counts were the objective rather than high resolution. Thus, it is instrumental broadening. The significant consequences of this broadening are that the feature at $100 \mathrm{~cm}^{-1}$ is hard to pick out, as it is largely lost in the tails of the much more intense substrate peaks, and that the low frequency cut off is at a higher point in order not to flood the CCD with broad tails of the Rayleigh scattered light. Second, the signal to noise ratio is much greater in the microscope configuration. This is due to the smaller optical losses in this configuration, as well as the larger numerical aperture of the microscope objective compared to the UV imaging lens. This is the main advantage of the microscope configuration. Thirdly, the features from the film are stronger in comparison to the substrate features in the microscope configuration. The ratio of film feature strength to substrate feature strength is greater in the nitrogen cryostat by a factor of approximately 2.5. This is an additional advantage 
of the microscope configuration, and is likely caused by the much shorter depth of field given by the microscope objective - illuminating with greater intensity the surface region where the STO is found than is possible with the conventional lens used in the He-cryostat configuration.

Using the microscope configuration enables us to continue to measure the strength of the polar modes as they become weak, and the temperature is raised above $250 \mathrm{~K}$. The high-temperature behaviour of the $\mathrm{TO}_{4}$ peak strength is shown in Fig. 6.10, with raw spectra shown in Fig. 6.11. Note that the amplitude of this peak does not drop to zero at high temperatures, but rather a small intensity remains even up to room temperature. At higher temperatures, the peak additionally becomes harder to see in the raw spectra because of the influence of the nearby stronger substrate peaks, which strengthen and broaden. The peak fitting can still pick them out, however. We interpret the reduction in intensity of this mode, as have many before us, not as an inherent weakness in the vibrational modes, but rather as a small volume of the film being under a polar distortion. The film volume under polar distortion is the only volume which is able to contribute to Raman scattering of the polar modes while STO is in cubic symmetry, hence the small measured intensity. In most previous reports, the polar regions are thought to be formed by locked in dipole moments of defects or grain boundaries polarising a region around them due to their electric field. The regions grow with decreasing temperature, as the polarisability of the bulk STO rises and thermal fluctuations decrease. These local polar microregions are in most cases randomly distributed. In the case of our films, however, there exists a clear gradient of strain across the depth of the film. As we know, high lattice strain is capable of raising the ferroelectric transition temperature of STO. So it is reasonable to expect that the STO layers that are closest to the film-substrate interface, and thus in a more highly strained condition will become polar regions before the STO layers closest to the film-air interface, where a significant amount of relaxation has occurred. We cannot assume, however, that our films are free from defects that may cause polar microregions in addition to the strain effect. Indeed, in some previous reports of thin films, it is assumed that polar microregions form around the dislocation defects that mediate the relaxation in strain. This would also lead to a higher concentration of polar microregions close to the film-substrate interface. It seems highly likely then, that the concentration of polar regions is greatest at 


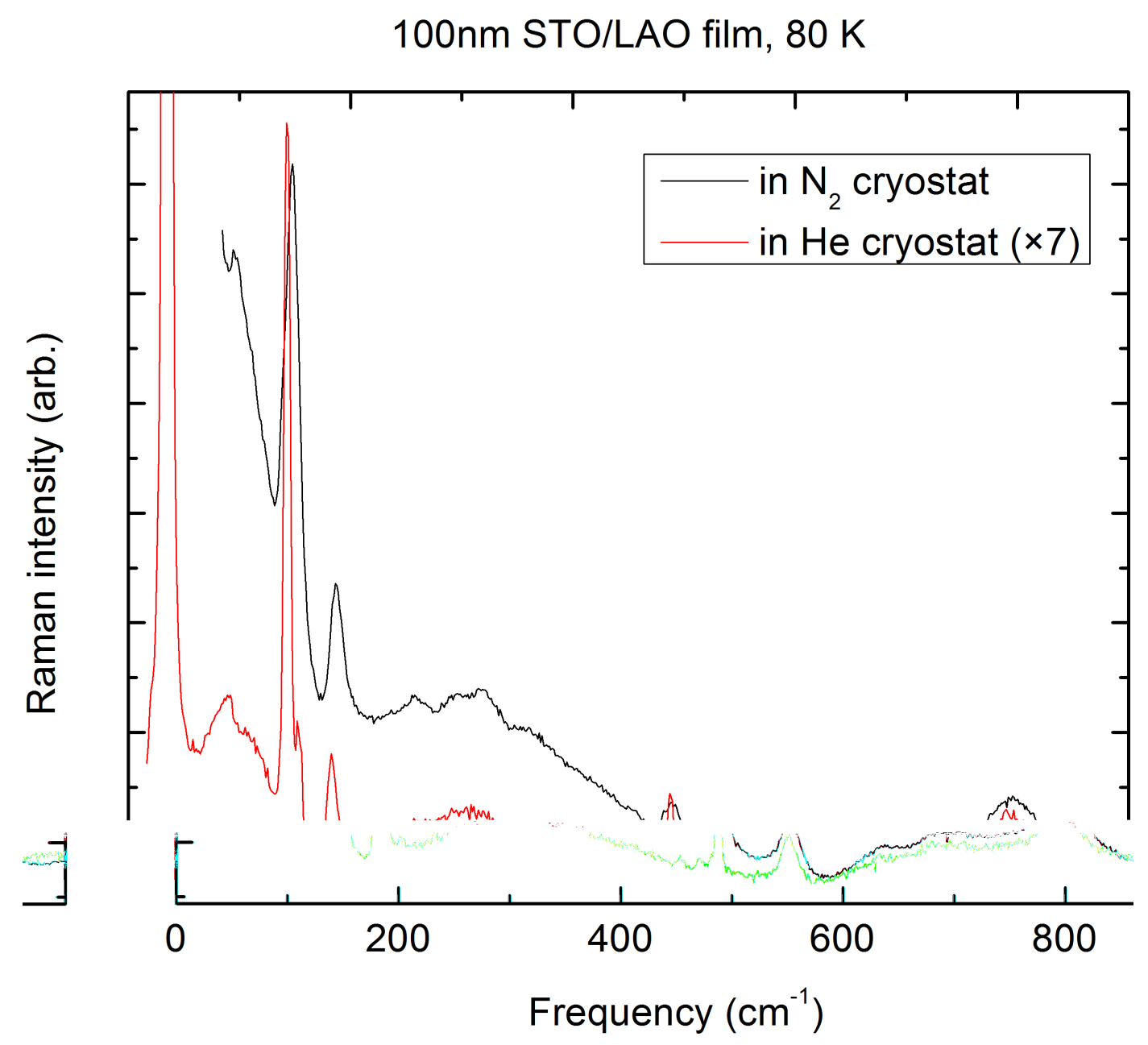

Figure 6.9: Raman spectra of sample STO267 at $80 \mathrm{~K}$ taken in two different experimental configurations - in black, the nitrogen cryostat and in red, the helium cryostat. There are some differences between the two spectra that can be attributed to experimental broadening, differences in throughput and differences in the volume being measured. 


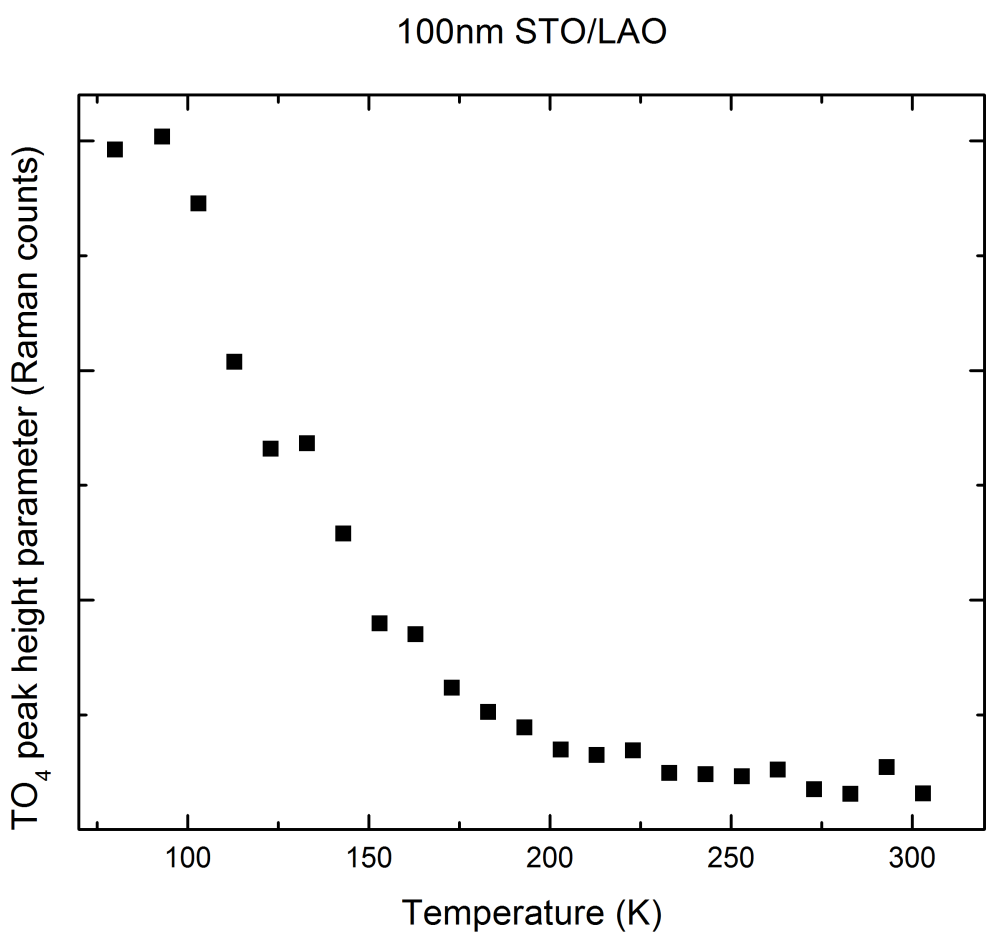

Figure 6.10: Temperature dependence of the $\mathrm{TO}_{4}$ peak height in sample STO267 as measured in the nitrogen cryostat. Note the long non-zero tail that remains above $200 \mathrm{~K}$, visible in this configuration due to the better surface selectivity.

the film-substrate interface, and decreases through the film thickness.

Fig. 6.12 shows the height of the $\mathrm{TO}_{4}$ mode with respect to temperature, measured in both of the different Raman configurations to show both low temperature and high temperature behaviour. The points from the low temperature measurements have been multiplied by a scale factor so that the two sets of points match in intensity over the region between $80 \mathrm{~K}$ and $200 \mathrm{~K}$ where both systems are able to make measurements. This alteration of the data is justified, as there is no reason to suspect that the two different experimental systems should have the same intensity of light incident on the sample, nor indeed the same efficiency between the sample and the CCD detection element. The scaling factor is here 9.5, giving a measure of the relative effectiveness of the two different optical configurations at measuring the STO thin film. The temperature dependence shown in this figure is the important information to take away from these measurements. 


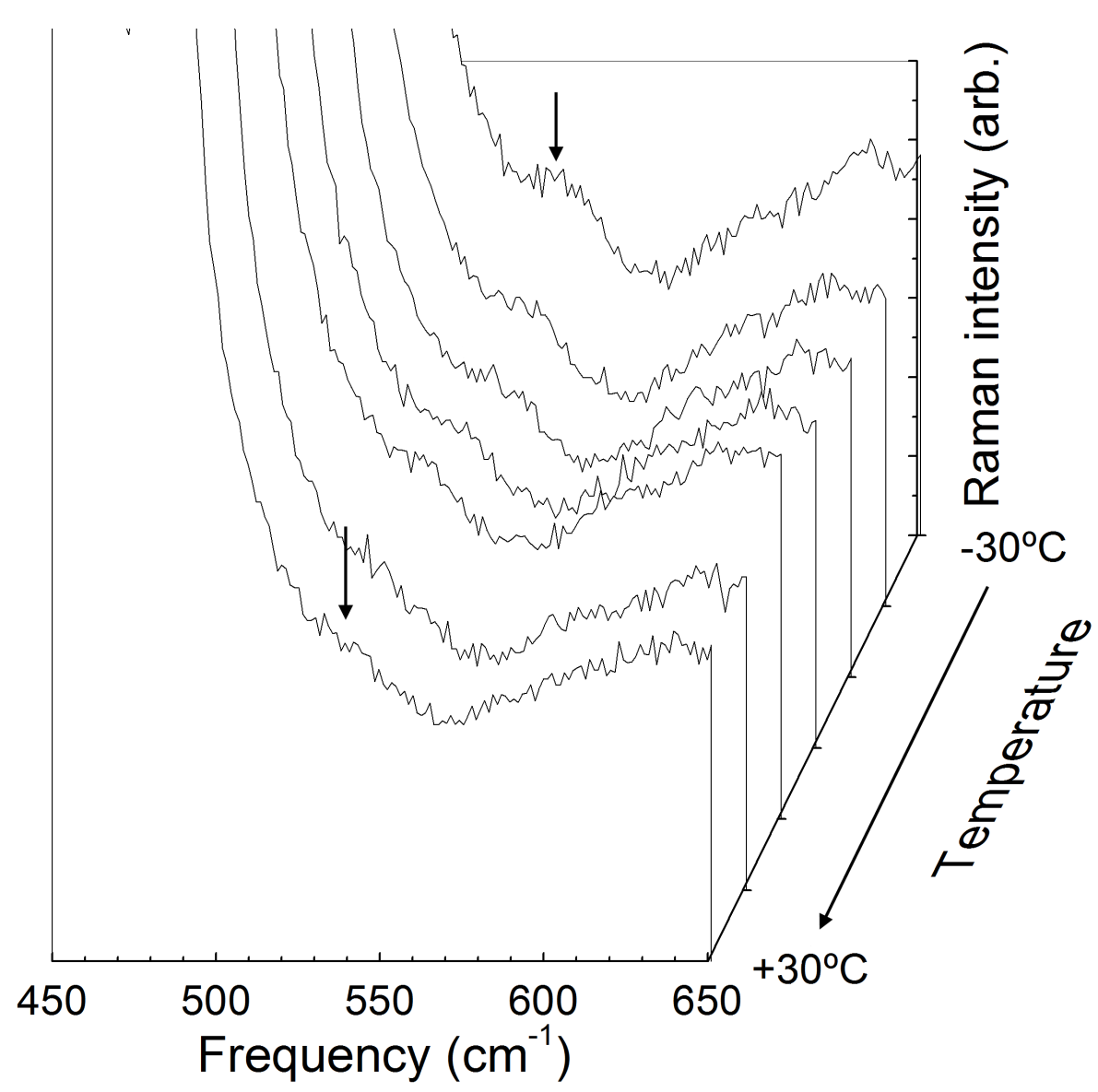

Figure 6.11: Temperature dependent Raman spectra of STO267 taken in the nitrogen cryostat showing the $\mathrm{TO}_{4}$ peak (marked with an arrow) remaining to high temperatures. The spectra have been offset both horizontally and vertically for ease of viewing 


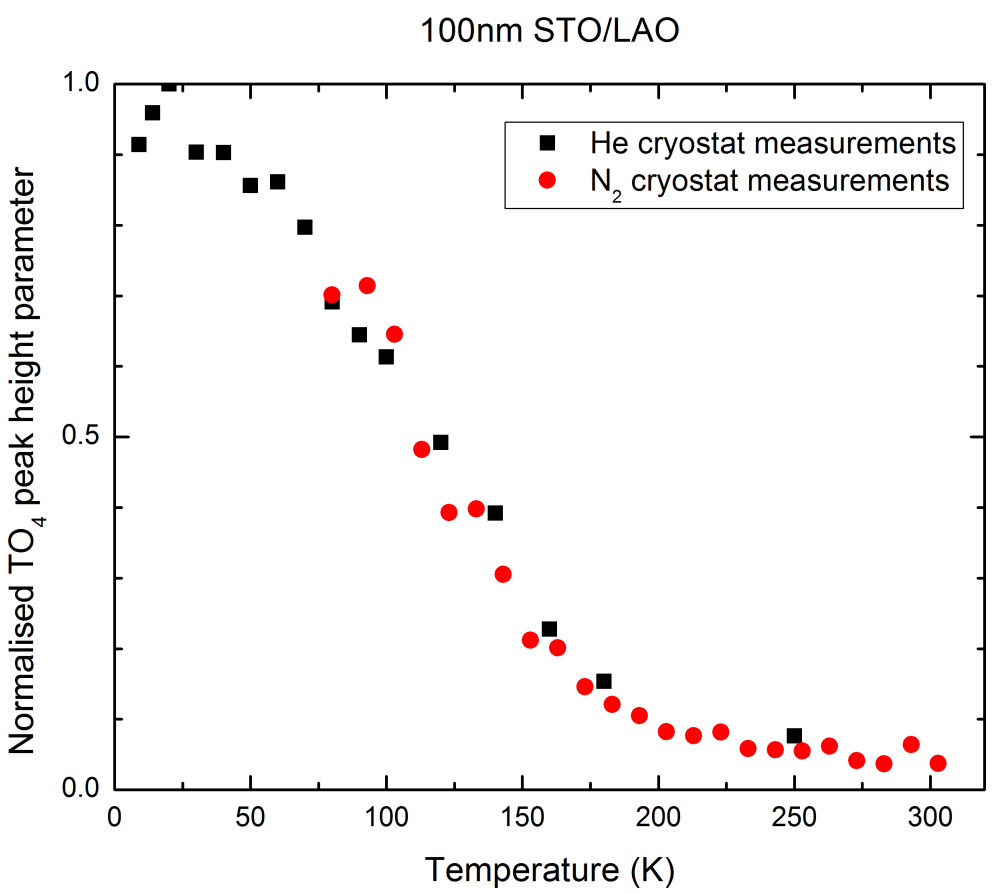

Figure 6.12: Temperature dependence of the $\mathrm{TO}_{4}$ peak height from measurements in both the nitrogen and helium cryostats, scaled to match each other between $80 \mathrm{~K}$ and $160 \mathrm{~K}$.

\subsubsection{Sample 263, $30 \mathrm{nmSTO/LAO:} \mathrm{spectra} \mathrm{in} \mathrm{helium} \mathrm{cryostat}$}

Fig. 6.13 shows the Raman spectrum of the thinner sample of STO/LAO $(30 \mathrm{~nm})$ at $9 \mathrm{~K}$ compared to that of the LAO substrate at $9 \mathrm{~K}$ collected in this configuration. As with the thicker layer, the strong peaks at $36,144,470$ and $487 \mathrm{~cm}^{-1}$ are present in both spectra, as is a weak peak at $709 \mathrm{~cm}^{-1}$. These features can be assigned to the substrate. Additional features are present in the STO sample spectrum. A square-shaped peak appears at $102 \mathrm{~cm}^{-1}$, which lies on top of a broader feature, and there are modes at $187 \mathrm{~cm}^{-1}$ and $553 \mathrm{~cm}^{-1}$ and $789 \mathrm{~cm}^{-1}$ corresponding to the $\mathrm{TO}_{2}, \mathrm{TO}_{4}$ and $\mathrm{LO}_{4}$ polar modes. The mode at $102 \mathrm{~cm}^{-1}$ judging by its shape is likely a plasma line. This could have gotten into the spectra if the plasma line filter had a slight deviation from orthogonality to the laser line. The broader excitation beneath it however is quite similar to the modes at 93 and $112 \mathrm{~cm}^{-1}$ in sample 267. Notably absent are the second order features expected from STO. The second order features are weaker by a factor of two or three than the polar modes in sample 267 (100 nm STO/LAO). However, a signal of this size should still be visible above the noise in the spectrum shown. This is an aspect of the 


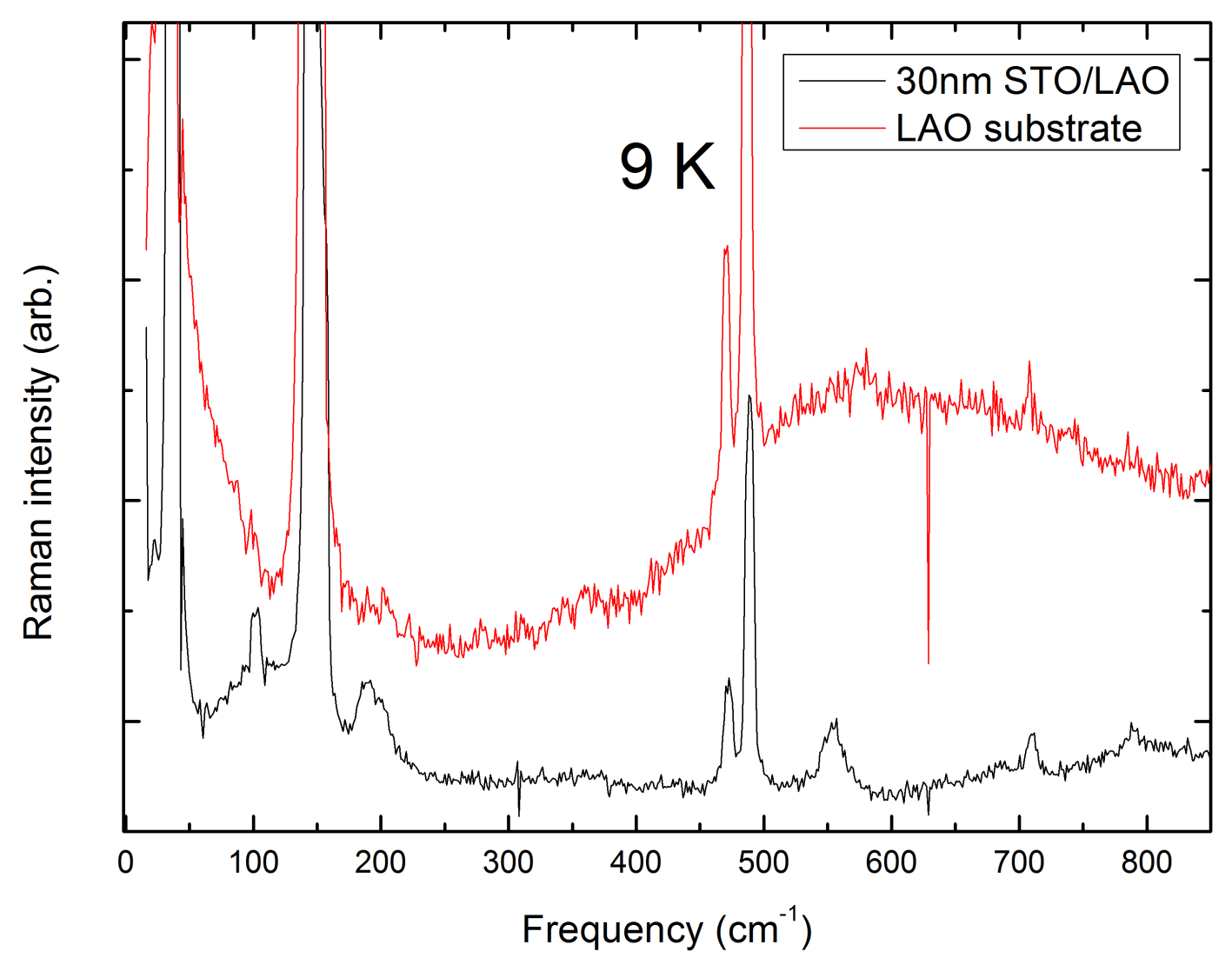

Figure 6.13: Raman spectra of sample STO263, and of the LAO substrate on which it is grown. Both spectra are taken at $9 \mathrm{~K}$, and this sample shows the same STO peaks that sample STO267 does, though they are weaker.

spectra that begs an explanation. Unlike the thicker layer, there is no peak in this spectrum at $450 \mathrm{~cm}^{-1}$. While the AFD soft modes and $150 \mathrm{~cm}^{-1}$ AFD hard modes would not be expected to be seen over the intense substrate lines, the absence of the $450 \mathrm{~cm}^{-1}$ line is clear. As with the thicker layer, XRD measurements have shown a clear superlattice peak appearing below $400 \mathrm{~K}$ proving the material to be in an AFD phase. Something about the films, possibly the small dimensions or possibly small amounts of Sr non-stoichiometry, causes these modes to be weak enough not to be seen in Raman spectra.

Fig. 6.14 compares the spectra of STO263 with the $100 \mathrm{~nm}$ thick layer STO267. One can see the absence of the second order features more clearly in this figure. Also of note is the difference in the shape of the $\mathrm{TO}_{2}\left(185 \mathrm{~cm}^{-1}\right)$ peaks between the two samples. Although substrate peaks and the $\mathrm{TO}_{4}$ peaks have similar widths across the samples, the full width half maximum of $\mathrm{TO}_{2}$ in 267 is $9.5 \mathrm{~cm}^{-1}$, whereas 


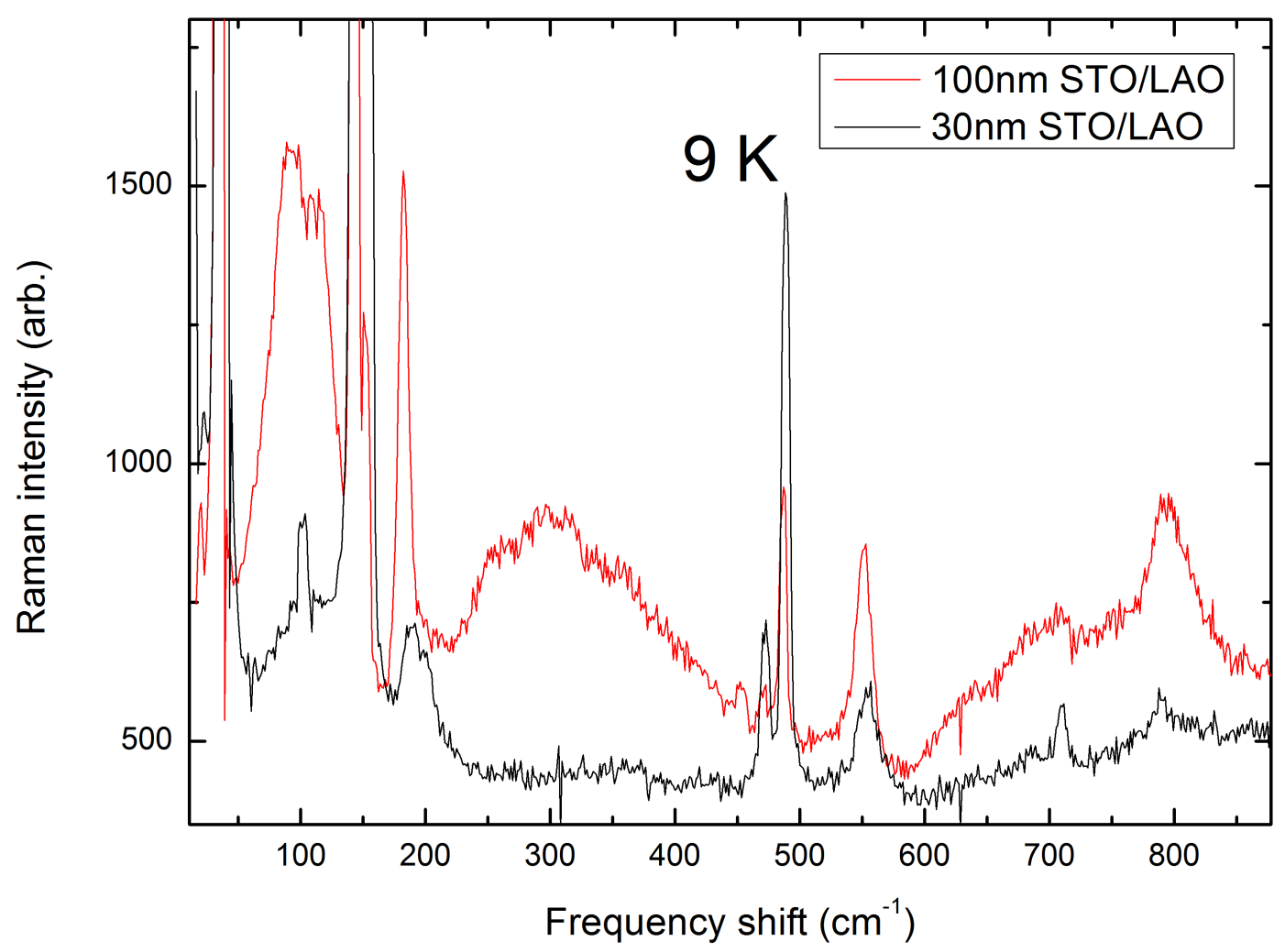

Figure 6.14: Comparison of the Raman spectra at $9 \mathrm{~K}$ of the $100 \mathrm{~nm}$ and $30 \mathrm{~nm}$ STO layers on LAO. The relative intensity of the different STO modes varies between the two samples.

in 263 it is $24 \mathrm{~cm}^{-1}$, broader by a factor of more than two. Since other lines are narrower than this one, and none of the other lines show any broadening in comparison to the $100 \mathrm{~nm}$ film, this cannot be an instrumental effect. It must be intrinsic to the mode itself.

Fig. 6.15 shows the height of the $\mathrm{TO}_{4}$ peak fitted by a Lorentzian function with respect to temperature, the fitting was done in the same way as for STO267; using spectra both in the He and $\mathrm{N}$ cryostats and scaling them to match over the temperature range accessible to both systems. Note that for this sample, the data are noisier than for the $100 \mathrm{~nm}$ thick layer. This is to be expected from a thinner film, giving lower signal to noise. The difference between the $100 \mathrm{~nm}$ layer and the $30 \mathrm{~nm}$ layer is striking - saturation of the polar mode height is reached at a much higher temperature of $80 \mathrm{~K}$ in the $30 \mathrm{~nm}$ layer - instead of $30 \mathrm{~K}$ in the $100 \mathrm{~nm}$ layer. The S-shaped curve is approximately the same breadth over all. Fig. 6.16 shows the peak height variation with temperature of both the $30 \mathrm{~nm}$ and $100 \mathrm{~nm}$ 


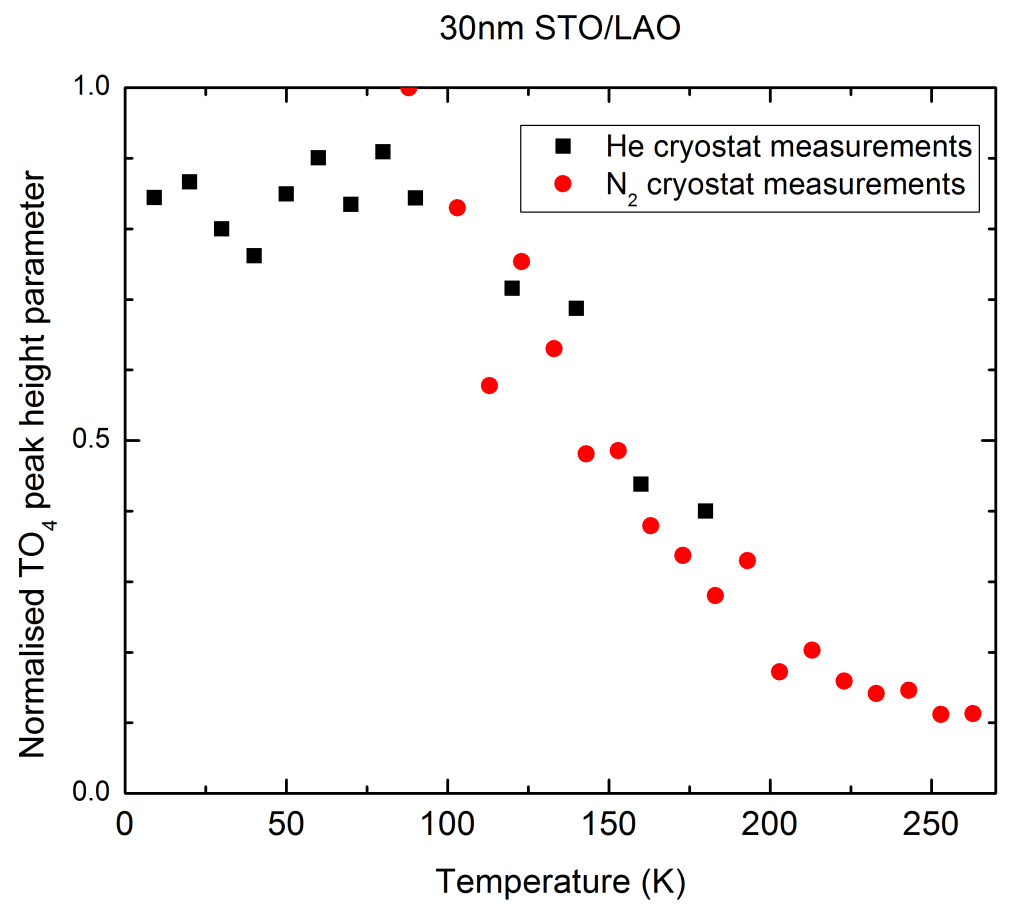

Figure 6.15: Temperature dependence of the $\mathrm{TO}_{4}$ mode in sample STO263, put together from the nitrogen and helium cryostat measurements as for the previous sample. In this sample, a saturation of the mode intensity at low temperatures is much more obvious.

layer side by side for comparison. The more or less horizontal translation of about $50 \mathrm{~K}$ from one to the other is obvious in this figure. The other factor of note is that the residual peak at high temperatures appears to be stronger relative to the saturation intensity in the $30 \mathrm{~nm}$ film than in the $100 \mathrm{~nm}$ one. Put another way, the high-temperature tail is thicker. One interprets this as a greater percentage of the film being in a high strain state in the $30 \mathrm{~nm}$ film.

\subsubsection{Samples STO263 and STO267: Overall intensity anomaly}

In both the STO263 and STO267 samples, there is an odd hysteresis displayed. Fig. 6.17 shows the spectra of the $100 \mathrm{~nm}$ layer STO267 in the microscope configuration. Note that both the substrate and the STO peak have a dip in intensity around $175 \mathrm{~K}$. This can be seen most clearly in the $550 \mathrm{~cm}^{-1}$ STO peak, and in the $140 \mathrm{~cm}^{-1}$ substrate peak. It was assumed that this dip was due to some anomaly in the reflectance of the sample at this temperature. During the peak fitting per- 


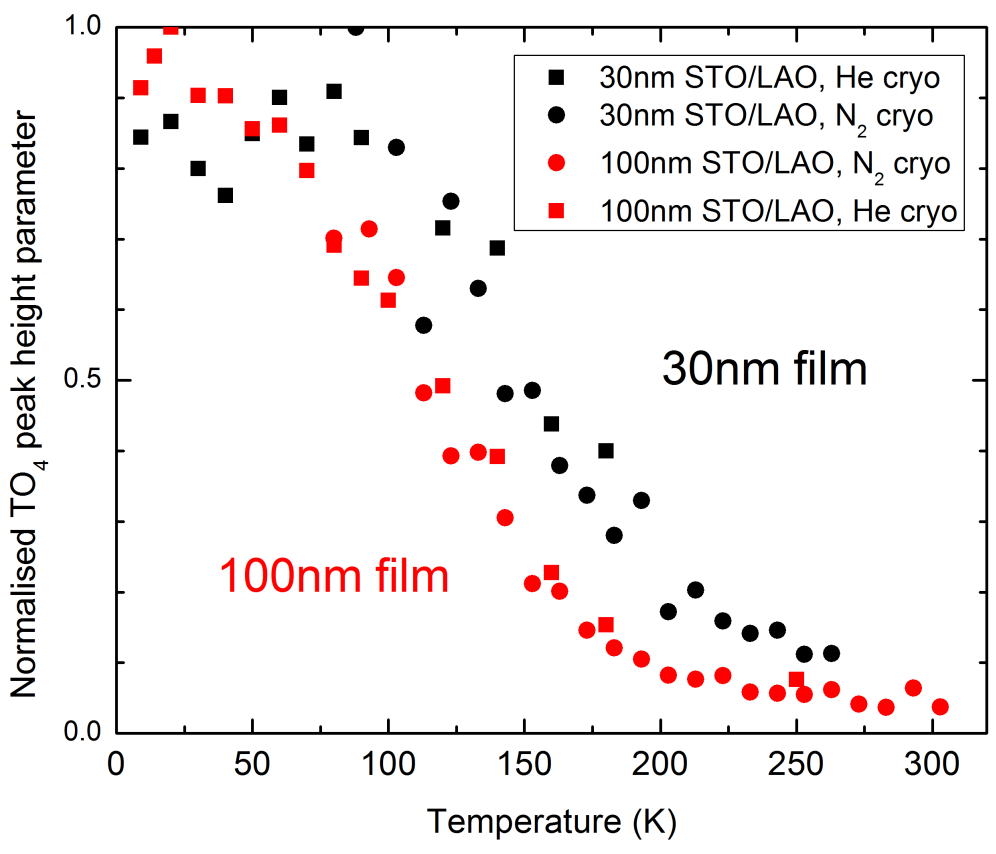

Figure 6.16: Temperature dependence of the $\mathrm{TO}_{4}$ modes of both STO on LAO samples. Measurements in both the nitrogen and helium cryostats are used to make this figure. The same pattern is seen in both samples, shifted $50 \mathrm{~K}$ higher in temperature in the thinner film, sample STO263.

formed on high temperature spectra, a substrate peak was fitted also to use as a reference signal, and the peak heights recorded and displayed in those graphs are peak heights corrected for this reduction in signal. It was later discovered that this dip in signal displayed hysteresis. Fig. 6.18 shows the height of fitted STO and substrate peaks a) taken with descending temperature and b) taken with rising temperature. Only the rising temperature spectra displayed this feature.

This anomaly in the Raman scattering seems to be correlated with the loss of first-order mode intensity in the sample, suggesting that the phenomenon has something to do with the disappearance of polar volumes in the film.

\subsubsection{Summary of results - STO on LAO}

In the films of STO grown on LAO, we have seen first order Raman modes gradually emerging and saturating in strength over a range of $170 \mathrm{~K}$. 


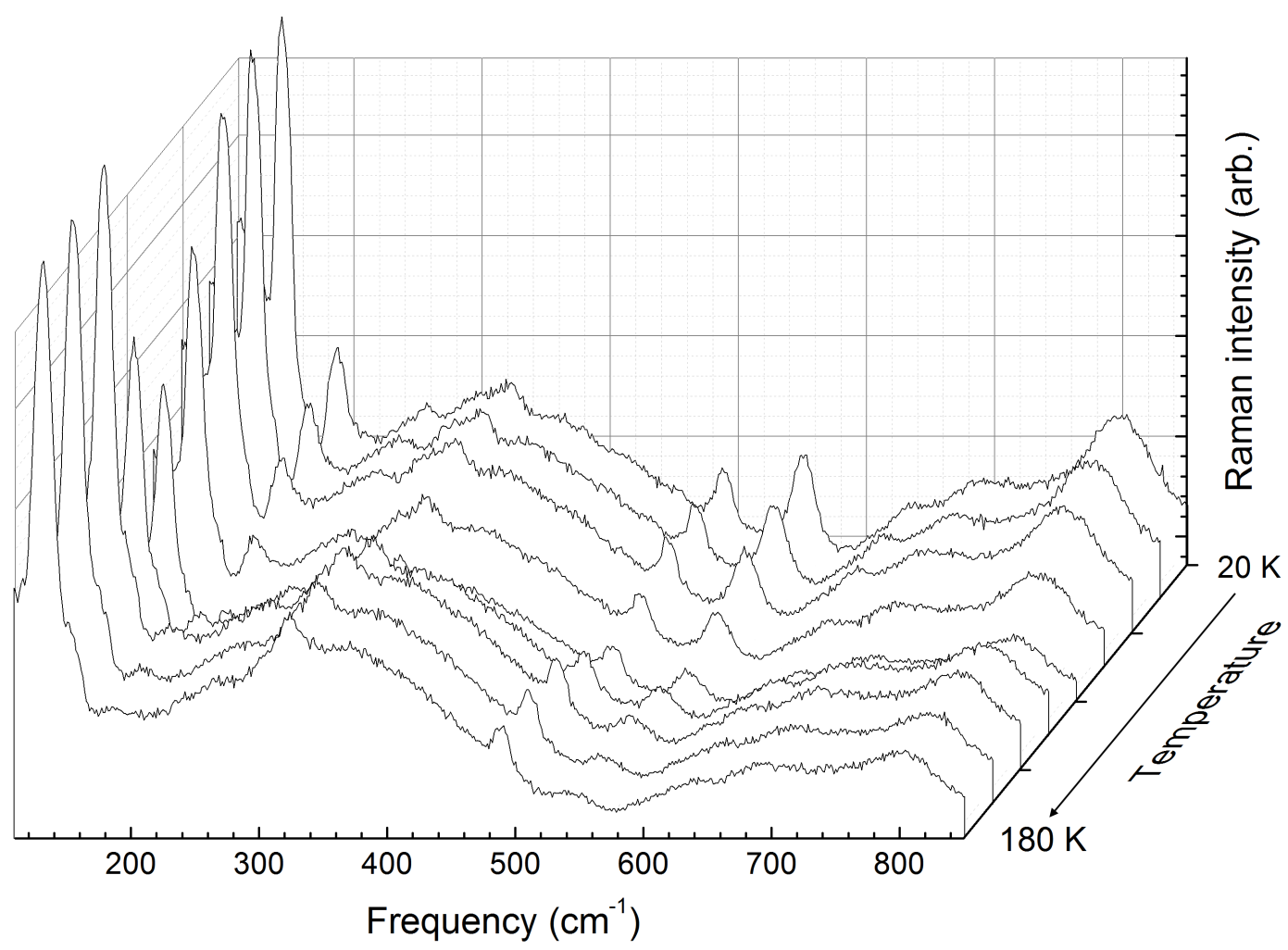

Figure 6.17: Temperature dependent Raman spectra of sample STO267 taken in the nitrogen cryostat. The spectra have been horizontally and vertically offset for ease of viewing. Note the dip in intensity of all modes - both STO and substrate - in the middle of the temperature series, at about $180 \mathrm{~K}$.

Sample STO263 is $30 \mathrm{~nm}$ thick, and the first order modes temperature dependence shows an S-shaped curve with width of about $170 \mathrm{~K}$, with saturation at $80 \mathrm{~K}$.

Sample STO267 is $100 \mathrm{~nm}$ thick, and the first order modes temperature dependence shows an S-shaped curve with width of about $170 \mathrm{~K}$. with saturation at $30 \mathrm{~K}$.

Both films also show a feature near $100 \mathrm{~cm}^{-1}$ which I could not confidently assign. This mode is considerably stronger in the thicker layer, both in comparison to the other STO modes, and in comparison to the substrate modes. In the thicker sample, this feature is split into two peaks and the lower-frequency one displays some temperature-dependent hardening. Further to this, there is a very weak mode at $450 \mathrm{~cm}^{-1}$ visible in the thicker layer. This may be an AFD hard mode (see section 2.3.2), indicating an AFD phase transition significantly raised from 


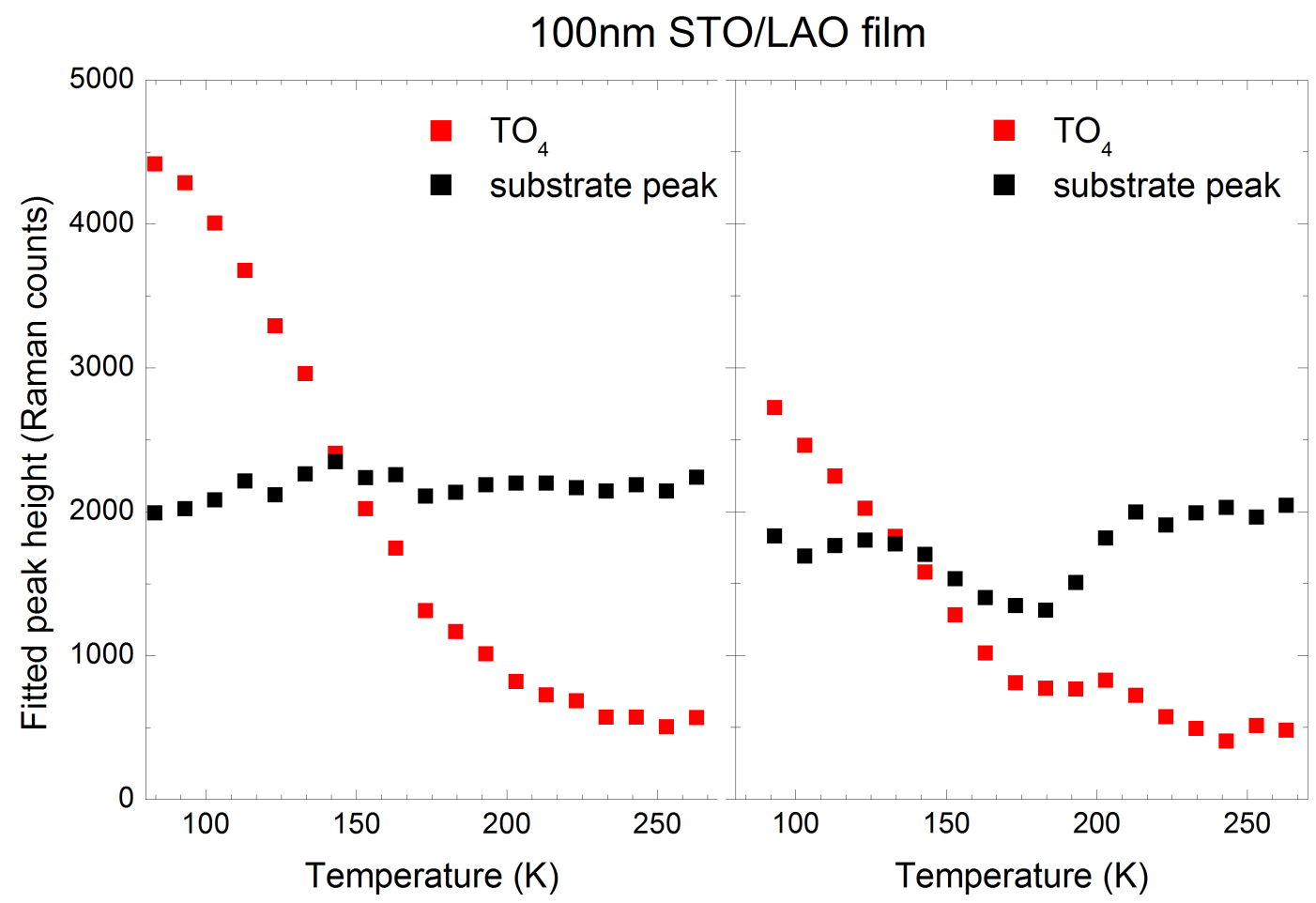

Figure 6.18: Peak heights of an STO and a substrate mode, variation with temperature. Left panel: Descending temperature. Right panel: ascending temperature. The dip in intensity of all modes is seen only with rising temperature. 
the unstrained case, and this result would agree with XRD measurements made on the samples.

\subsection{STO on NGO}

The samples in this section were grown on $\mathrm{NdGaO}_{3}$ (NGO) [110]. This surface of NGO is not square, having lattice mismatch with STO of $-1.0 \%$ and $-1.3 \%$ (compressive) on its two axes.

\subsubsection{Sample STO264: $50 \mathrm{~nm}$ STO/NGO: spectra in helium cryostat}

Fig. 6.19 shows spectra of STO264 and NGO substrate collected in the helium cryostat configuration at $9 \mathrm{~K}$. Clearly, there are a great number of substrate peaks present in both spectra. They are at: 93, 149, 172, 184, 208, 220, 297, 344, $356,413,430,465$, and $553 \mathrm{~cm}^{-1}$. Some differences in the relative frequencies of these substrate peaks between the substrate and the film are expected because the NGO and STO/NGO are two physically different samples, therefore polarisation is most likely not the same in the two spectra. There are a few features present in the film spectrum which are not present in the NGO spectrum, and can be used as indicators of the STO film. First, there is a pair of strong peaks at very low frequency: 31 and $38 \mathrm{~cm}^{-1}$ respectively. Then, there is also a weak peak at $267 \mathrm{~cm}^{-1}$. Finally, there is a broad feature between $700-800 \mathrm{~cm}^{-1}$, which is made up of second order features sitting under a broad peak centered at $792 \mathrm{~cm}^{-1}$. The $792 \mathrm{~cm}^{-1}$ peak is of the right position and shape to be the $\mathrm{LO}_{4}$ polar hard mode of STO. The small peak at $267 \mathrm{~cm}^{-1}$ is at the right position, and of approximately the right intensity to be the $\mathrm{TO}_{3}$ non-polar hard mode of STO - this peak has always been weak in comparison to the other polar modes in literature reported previously. The features at 31 and $38 \mathrm{~cm}^{-1}$ cannot be unambiguously assigned from this spectrum alone. Peaks present in STO at approximately this frequency include both AFD soft modes, and the ferroelectric soft mode. The temperature dependence of these peaks will give us more information to work with.

In the analysis of the STO films grown on LAO, we made heavy use of the $\mathrm{TO}_{4}$ 


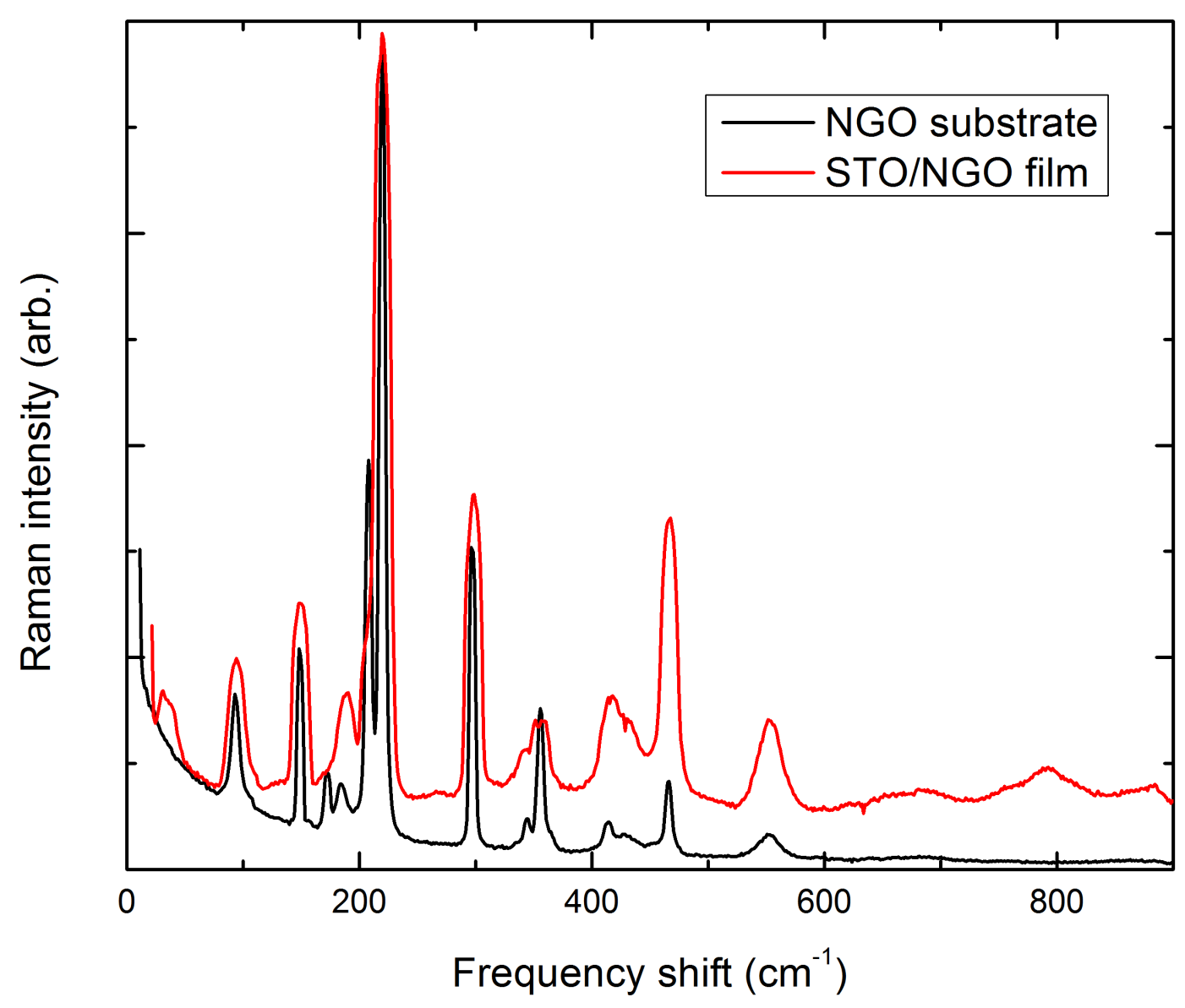

Figure 6.19: Raman spectra of sample STO264 compared to the NGO substrate on which it is grown. In this case, there are very few modes which are seen in the sample and not the substrate - a feature near $40 \mathrm{~cm}^{-1}$ and one at $800 \mathrm{~cm}^{-1}$. Note that this spectrum of STO on NGO has a lower resolution $-10 \mathrm{~cm}^{-1}$ rather than the usual $5 \mathrm{~cm}^{-1}$. 
peak, tracking its temperature dependence to discover the nature of the phase transition and the ferroelectricity of the film. In this sample grown on NGO however, there are peaks in the substrate sitting right over $\mathrm{TO}_{4}$, and also over $\mathrm{TO}_{2}$ which will make analysis using these peaks problematic. Fig. 6.20 shows temperature dependent spectra of the NGO substrate. Notice here that not only does the substrate have peaks at $185 \mathrm{~cm}^{-1}$ and $550 \mathrm{~cm}^{-1}$ overlapping directly with the $\mathrm{TO}_{2}$ and $\mathrm{TO}_{4}$ polar hard modes in STO, those peaks in the substrate are also strongly temperature dependent. Even with substrate subtractions, the data are not clean enough on these two peaks to justify using them as any kind of indicator of STO. However, the $\mathrm{LO}_{3}$ hard polar mode of STO at about $800 \mathrm{~cm}^{-1}$ does not overlap with any substrate features in this sample, and should in principle be able to be used for our analysis. Also significant is the $\mathrm{TO}_{3}$ peak, absent from the STO/LAO samples. As noted in the literature, this peak is silent in the Raman in cubic and tetragonal phases of STO, and Raman active in orthorhombic ferroelectric STO. And whereas other TO and LO modes often are active in nonferroelectric precursor phases such as polar microregions, $\mathrm{TO}_{3}$ is activated only seldom in them. If this is indeed $\mathrm{TO}_{3}$ we are seeing, it is a stronger suggestion of a centrosymmetry breaking that extends cohesively over a long range. Both these peaks can be used to determine the nature of the crystal structure in this film.

Fig. 6.21 shows the change in the $792 \mathrm{~cm}^{-1} \mathrm{LO}_{4}$ peak with temperature. Note the sudden appearance of this peak between $160 \mathrm{~K}$ and $140 \mathrm{~K}$, a noticeably different temperature dependence than was present in the STO/LAO thin films. In those samples, the appearance of the polar modes was gradual over a period of about $100 \mathrm{~K}$. Another difference is the fact that the amplitude of this peak does not monotonically increase as the temperature is lowered. Below $30 \mathrm{~K}$, the amplitude of the peak is noticeably smaller than between $100 \mathrm{~K}$ and $30 \mathrm{~K}$. Fig. 6.22 shows the temperature dependent raw spectra of the $267 \mathrm{~cm}^{-1}$ feature, suspected to be $\mathrm{TO}_{3}$. It is the small peak in the figure between two larger substrate peaks. The mode starts appearing below $150 \mathrm{~K}$, increasing in intensity down to to the lowest temperature measured, $10 \mathrm{~K}$. No measurable change in frequency is displayed. At temperatures above those shown, the increasing width of the substrate peaks drowns out the decreasing signal from the $\mathrm{TO}_{3}$ peak.

Fitting the $\mathrm{LO}_{4}$ peak is a more difficult process than the $\mathrm{TO}_{2}$ and $\mathrm{TO}_{4}$ peaks in the previous samples. Whereas those peaks were sharp and more or less free from 


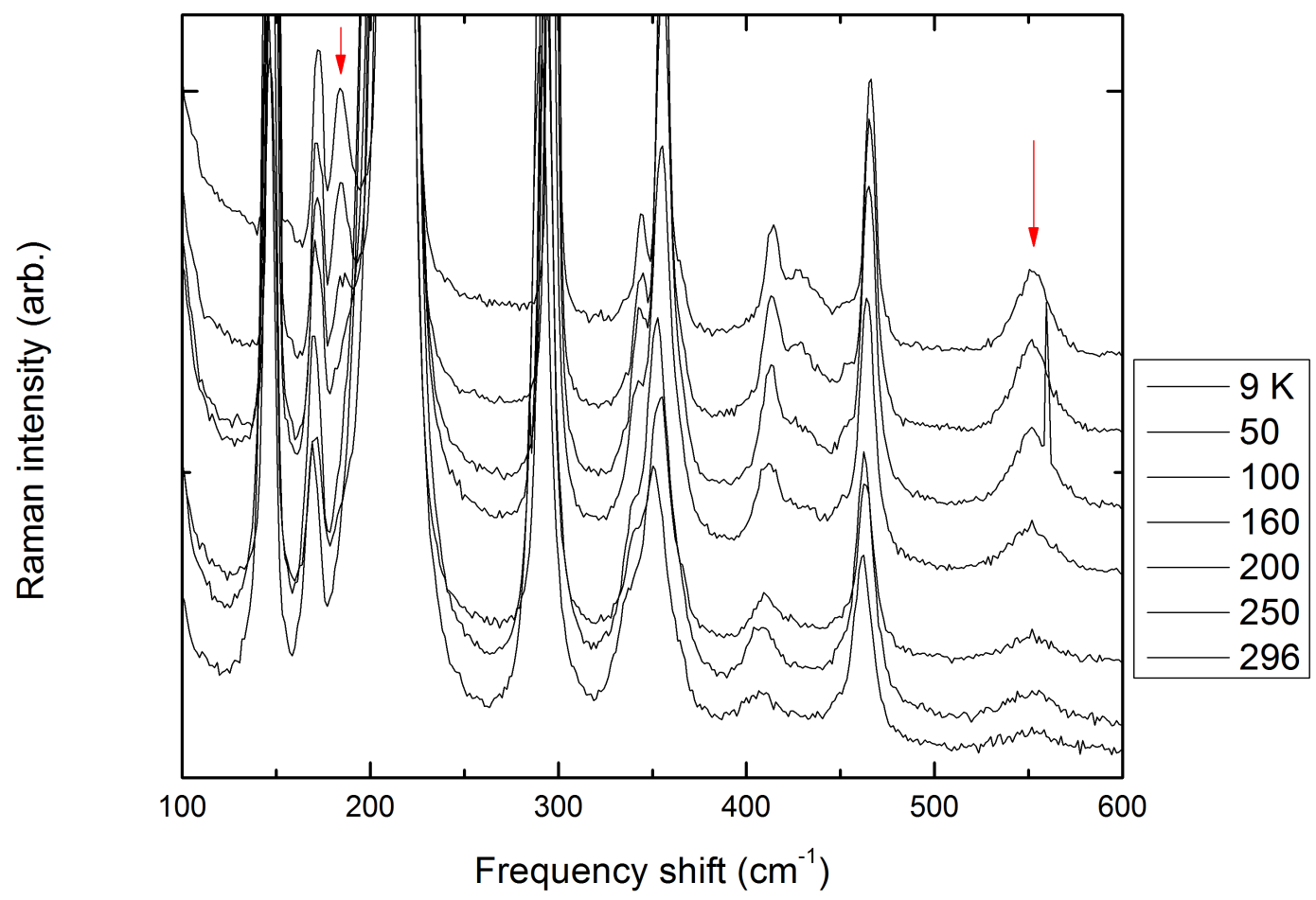

Figure 6.20: Temperature dependent Raman spectra of NGO substrate. The spectra have been vertically offset for ease of viewing. The peaks at $180 \mathrm{~cm}^{-1}$ and $550 \mathrm{~cm}^{-1}$ marked with arrows not only have the same frequencies as the the $\mathrm{TO}_{2}$ and $\mathrm{TO}_{4}$ modes of STO, but they also show a very similar variation with temperature. It will be very difficult to use these peaks in the sample to infer anything about STO. 

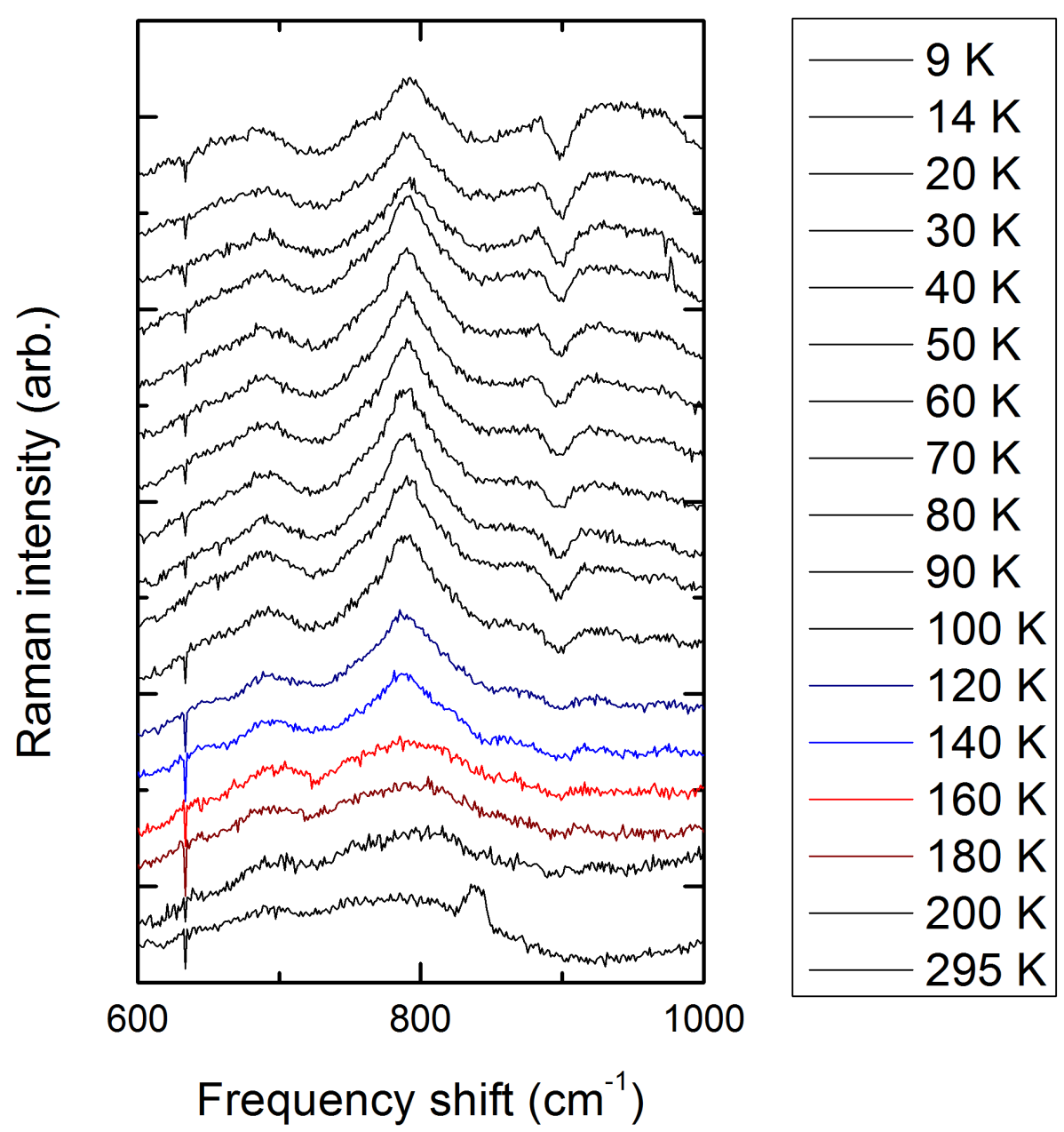

Figure 6.21: Temperature dependent Raman spectra of sample STO264, close up on $\mathrm{LO}_{4}$ peak. The spectra have been vertically offset for ease of viewing. The peak changes more sharply with temperature in this sample than in the other STO samples studied so far. 


\section{Appearance of peak at $267 \mathrm{~cm}^{-1}$ in STO/NGO}

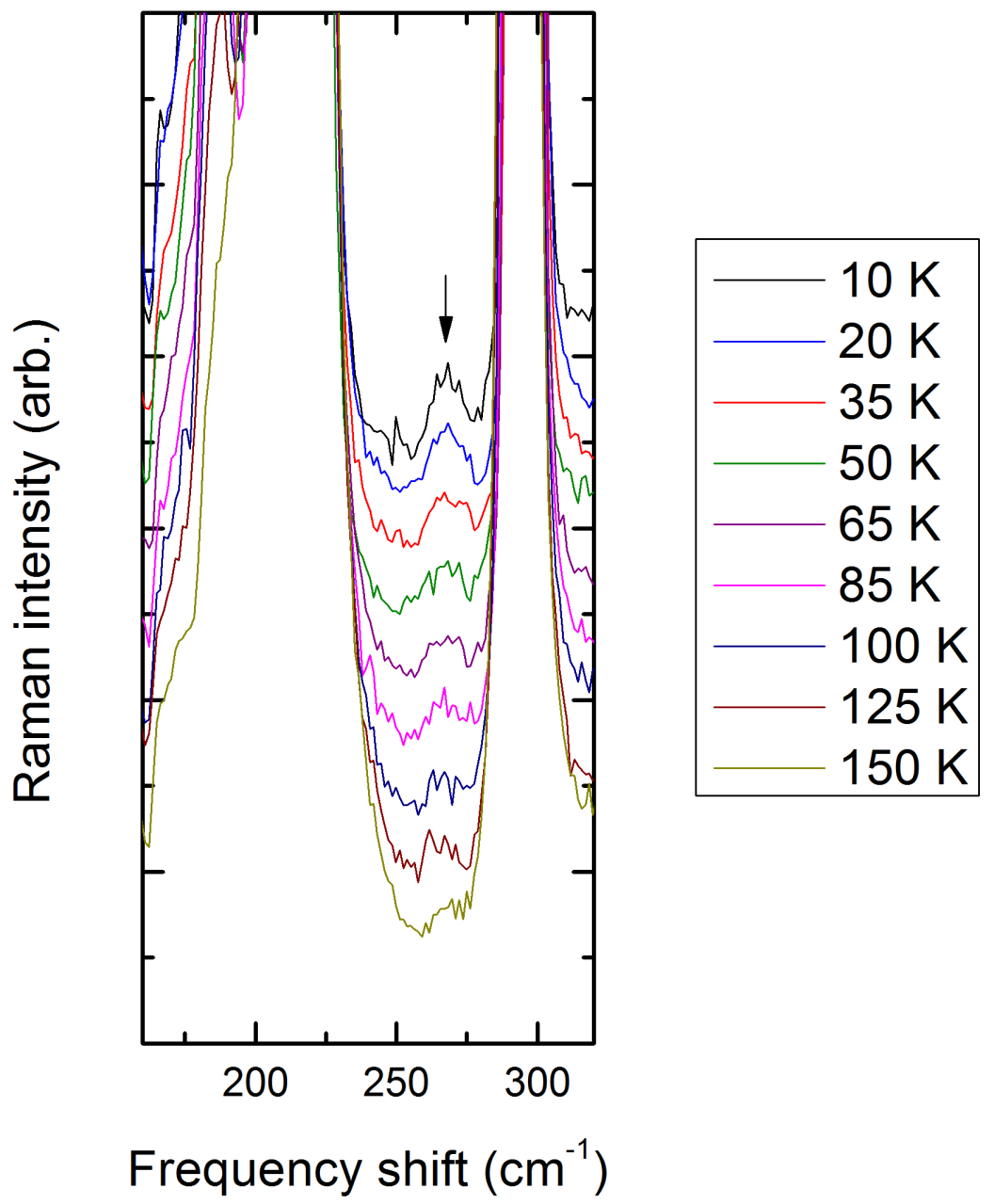

Figure 6.22: Temperature dependent Raman spectra of sample STO264, close up on the $\mathrm{TO}_{3}$ peak. The spectra have ben vertically offset for ease of viewing. This peak can be seen emerging from above substrate lines from about $159 \mathrm{~K}$. In this sample, $\mathrm{TO}_{3}$ has a different temperature dependence than the other TO and LO modes in STO. 
interference by other signals, the $\mathrm{LO}_{4}$ peak in this sample is broad and sits on top of a broader excitation. The broad excitations on which it sits are second order features from STO itself, so the $\mathrm{LO}_{4}$ peak can never be perfectly isolated. Fig. 6.23 shows several different results of peak fitting of various kinds, which I will discuss here. As can be seen from the raw spectra, there is a dramatic change in this feature between $140 \mathrm{~K}$ and $160 \mathrm{~K}$. When a single Lorentzian line is fitted to the spectrum between 720 and $860 \mathrm{~cm}^{-1}$, the height parameter plotted in black squares is obtained (the comments made in section 6.2.1 regarding height vs amplitude for peak fitting are still relevant). The height is maximum at $80 \mathrm{~K}$, and it shows a drop to either side. At $140 \mathrm{~K}-160 \mathrm{~K}$, the height stops changing, becoming constant at a value of roughly 700 counts. However, the fit obtained is not good if one examines the fitted peak and the spectrum. The fitted line matches relatively well the data within the $720-860 \mathrm{~cm}^{-1}$ range, but very poorly models the data outside this range. The width of the peak becomes too large, the baseline $\mathrm{Y}_{0}$ value too high. Plotted in Fig. 6.23 in the blue circles is the width parameter of the fitted peak (scaled to fit into the figure area). One can see that the width suddenly increases by a factor of more than two between $140 \mathrm{~K}$ and $180 \mathrm{~K}$, an indication of a change in the spectrum. If one examines closely the raw spectra, one might believe that the $\mathrm{LO}_{4}$ peak truly has become broader. Alternately, one could view what is left as the broad excitation upon which the $\mathrm{LO}_{4}$ sits, and interpret the sudden change in fitting parameters as the disappearance of the $\mathrm{LO}_{4}$ peak entirely, and the fitting function starting to fit to the underlying broad feature. Either way, it is a significant change, and one which has in the literature been associated with the disappearance of polar regions in STO. The third data plotted in Fig. 6.23 is the height parameter of a second round of fitting, this time in which the width parameter is restricted to be less than or equal to $55 \mathrm{~cm}^{-1}$. This treatment shows more clearly the sudden change in character between $140 \mathrm{~K}$ and $160 \mathrm{~K}$ in a way that can be compared with the other samples.

Fig. 6.24 shows this temperature dependence alongside the temperature dependence of the fitted $\mathrm{TO}_{3}\left(267 \mathrm{~cm}^{-1}\right)$ peak height, obtained from long-integration measurements. Alongside this the temperature dependence of the $\mathrm{LO}_{4}$ peak discussed earlier is displayed. Compare this to Fig. 6.22 reproduced from Toulouse et al.[2] showing the temperature dependence of $\mathrm{TO}_{3}$ and $\mathrm{TO}_{2}$ in $\mathrm{KTa}_{1-\mathrm{x}} \mathrm{Nb}_{\mathrm{x}} \mathrm{O}_{3}$ a non-polar TO mode and a polar TO mode respectively (The figure is duplicated 


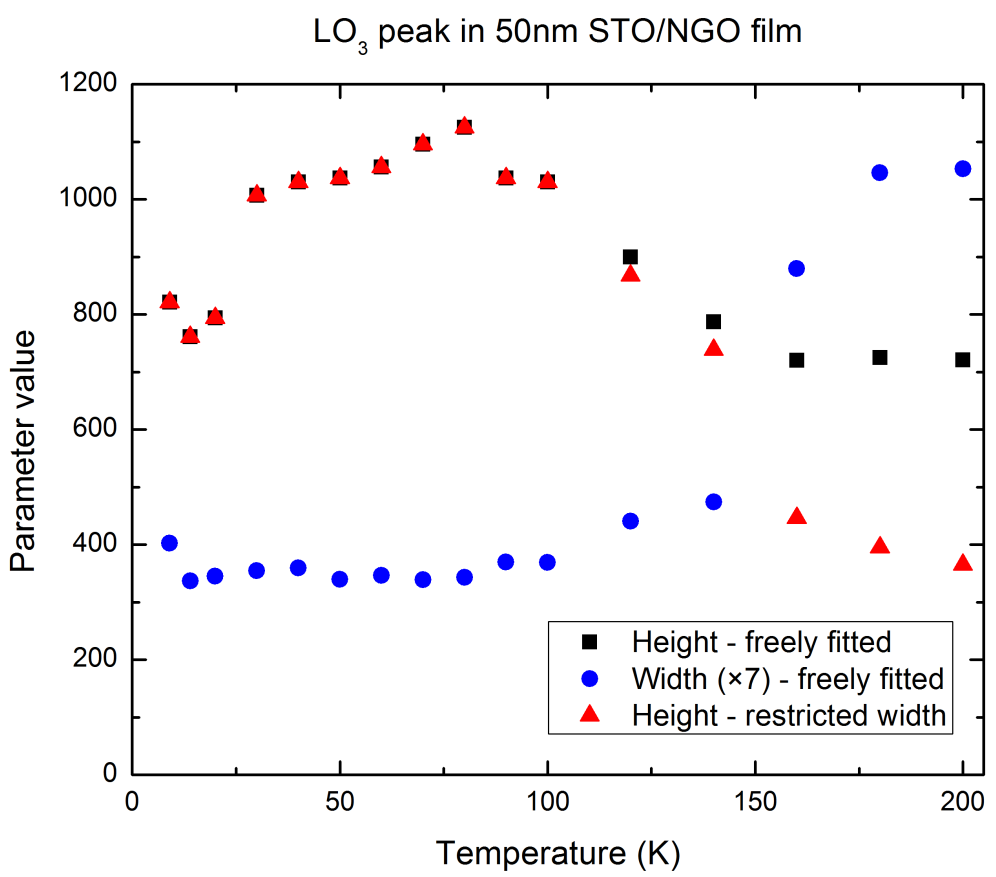

Figure 6.23: Figure showing fitting parameters of $\mathrm{LO}_{4}$ peak and underlying second order features. In black squares: fiting with all parameters free. Above $150 \mathrm{~K}$ the model becomes poor. This can be seen in the rapidly rising width parameter, blue circles. In red triangles is fitting with the width parameter constrained. The result is a better, though still not perfect representation of the $\mathrm{LO}_{4}$ peak intensity.

here for ease of viewing). In this material, in the $1.2 \%$ substitution case there is a peak in the dielectric permittivity at $15 \mathrm{~K}$, and no peak seen in the $1 \%$ case. The permittivity peak coincides with the appearance of the $\mathrm{TO}_{3}$ mode, and a sharp reduction in the intensity of the $\mathrm{TO}_{2}$ mode. Similar temperature dependence of the polar $\mathrm{LO}_{4}$ and non-polar $\mathrm{TO}_{3}$ Raman features seen in the $1.2 \% \mathrm{KTN}$ sample is present in this STO sample, albeit more spread out in temperature than in the KTN case. We see a drop in intensity of the polar $\mathrm{LO}_{4}$ mode at the same temperature as the non-polar $\mathrm{TO}_{3}$ mode starts to grow from zero intensity. Although of course I do not have a direct measurement of the permittivity to confirm or deny ferroelectricity in this STO thin film, the polar and non-polar mode behaviour seen in the Raman is consistent with a ferroelectric material.

Fig. 6.26 shows the change in the low frequency feature with respect to temperature. Note the splitting from one peak into two between $160 \mathrm{~K}$ and $140 \mathrm{~K}$ - the same temperature at which the $\mathrm{LO}_{4}$ mode appears. It seems highly likely given the splitting and the frequency of these peaks that they are components of 


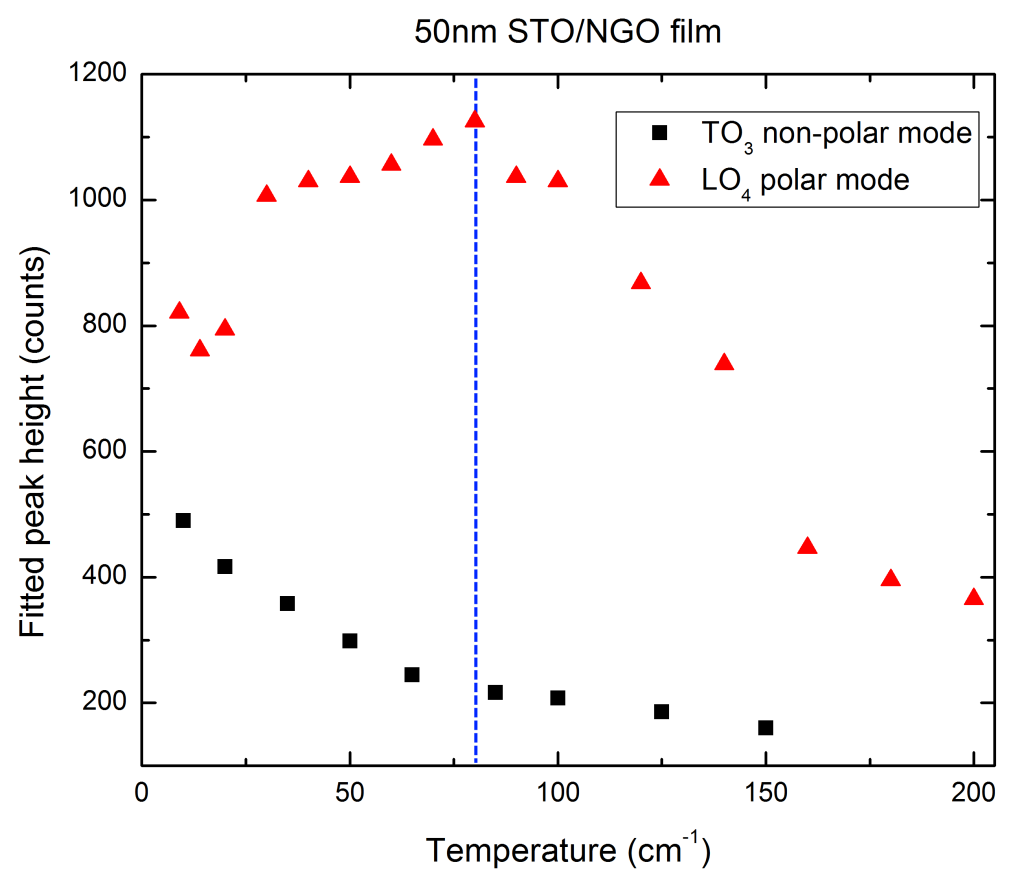

Figure 6.24: Temperature dependence of vibrational mode intensities in sample STO264. Note the difference between the $\mathrm{TO}_{3}$ and $\mathrm{TO}_{4}$ modes, and compare the changes at the dotted blue line in this figure to the changes at $50 \mathrm{~K}$ in Fig. 6.25.

the AFD soft mode. In pure unstrained STO, this mode would not be seen above $110 \mathrm{~K}$, but XRD of this sample shows evidence of a structural phase transition at about $400 \mathrm{~K}$. While the character of this transition could not be determined by XRD alone due to interference from substrate peaks, the AFD is typically the highest temperature distortion shown by STO. It is then entirely reasonable to expect that one could see the AFD mode in the Raman spectra at 140-160 K, and I interpret this peak as such. The frequency of these modes is somewhat higher than in the Ca-substituted or ${ }^{18} \mathrm{O}$-substituted single crystals, and the peaks are broader as well[43][38].

The implication of the splitting of this mode is that there is a transition to a lower symmetry phase at around $160 \mathrm{~K}$ in this sample, probably to orthorhombic. There is evidence in the XRD of this sample of a second structural phase transition at this temperature. With the help of the Raman spectra showing a rapid rise in intensity of the polar $\mathrm{LO}_{4}$ mode, the emergence of the $\mathrm{TO}_{3}$ mode simultaneously with a drop of intensity of the polar $\mathrm{LO}_{4}$ mode, and the splitting of the AFD soft mode, we can confidently assign it to the ferroelectric phase transition. 


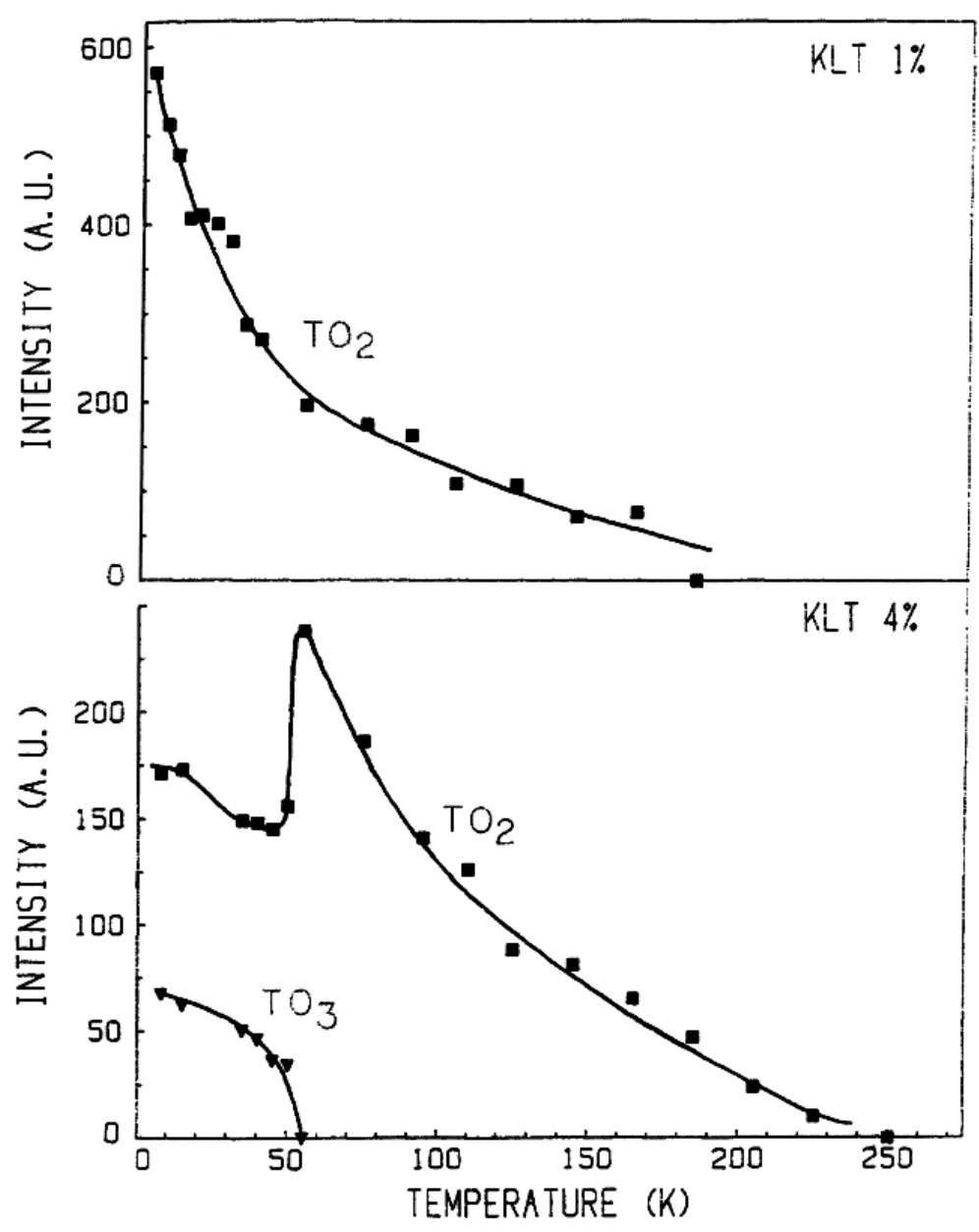

Figure 6.25: Temperature dependence of first-order scattering in KLT. Above: 1\% Li, Below: $4 \%$ Li. the $4 \%$ KLT has a ferroelectric phase transition at about $50 \mathrm{~K}$, and one can see the spectral changes associated with it. Similar effects are seen in the Raman spectra for STO264 - see Fig. 6.24. 1\% KLT is not ferroelectric. Figure reproduced with permission from [2]. 
Low frequency peak splitting in STO/NGO

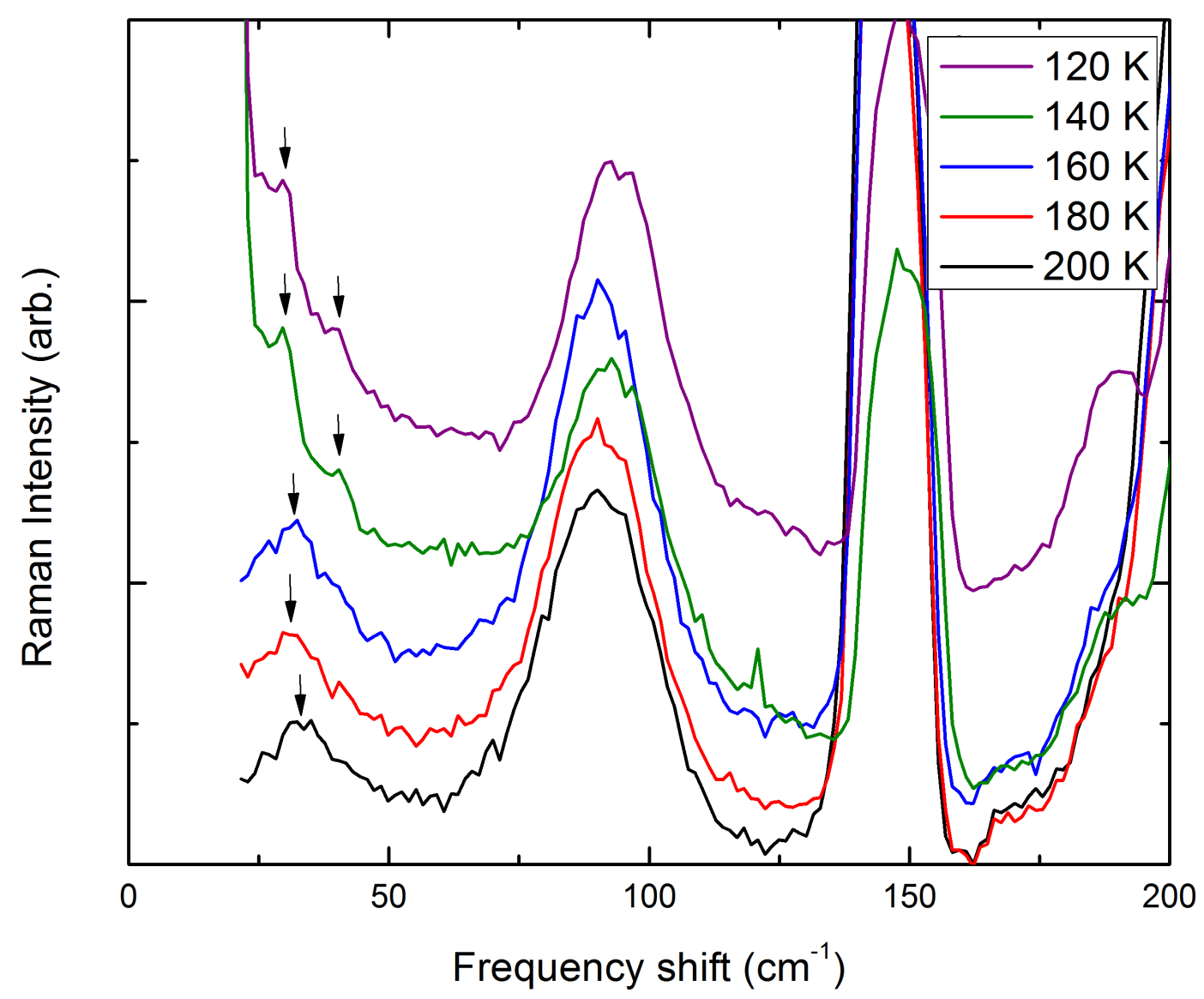

Figure 6.26: Temperature dependent Raman spectra of the $35 \mathrm{~cm}^{-1}$ feature in sample STO264. The spectra have been vertically offset for ease of viewing. Note the splitting of one peak into two peaks accompanied by a sharp increase in lowfrequency scattering between $160 \mathrm{~K}$ and $140 \mathrm{~K}$, as marked by the arrows. 


\subsubsection{Summary of results - STO on NGO}

Sample STO264 shows the polar hard mode $\mathrm{LO}_{4}$ above the substrate in the Raman spectra. Its temperature dependence shows an S-shaped curve with width of about $80 \mathrm{~K}$, with saturation occuring at $80 \mathrm{~K}$.

In addition, two other features of STO are seen. We see evidence in the Raman spectra of a splitting of the AFD soft mode feature below about $150 \mathrm{~K}$. We also see appearance of the $\mathrm{LO}_{4}$ hard mode at this temperature, followed at around $80 \mathrm{~K}$ by the appearance of the $\mathrm{TO}_{3}$ mode simultaneously with a sharp drop in intensity of the $\mathrm{LO}_{4}$ mode. Both of these are features that are, in the literature associated with a ferroelectric phase transition. On this basis, I am confident in assigning a structural phase transition from tetragonal to orthorhombic between $160 \mathrm{~K}$ and $140 \mathrm{~K}$ in this sample, with ferroelectricity displayed by the orthorhombic phase as is usual for the STO system.

\subsection{STO on LSAT}

The samples in this section were grown on $\left(\mathrm{LaAlO}_{3}\right) \cdot\left(\mathrm{SrAl}_{0.5} \mathrm{Ta}_{0.5} \mathrm{O}_{3}\right)(\mathrm{LSAT})$ [001], lattice mismatch of $-0.95 \%$ (compressive strain) with STO

\subsubsection{Sample STO006: $105 \mathrm{~nm}$ STO/LSAT measured in helium cryostat}

Fig. 6.27 shows spectra of the film, and of the substrate measured at $10 \mathrm{~K}$ in the helium cryostat. The substrate has many features, all of which show up in the thin films measurements. The second order features, $\mathrm{TO}_{2}, \mathrm{TO}_{4}, \mathrm{LO}_{4}$ modes and a similar feature at around $100 \mathrm{~cm}^{-1}$ to that found in the STO/LAO films are all seen above the substrate lines in this sample. The shape of the peaks is very similar - although one should not discount the influence of the substrate in this sample - and the frequency is in this case slightly lower, at $77 / 97 \mathrm{~cm}^{-1}$ rather than $93 / 112 \mathrm{~cm}^{-1}($ at $10 \mathrm{~K})$. 


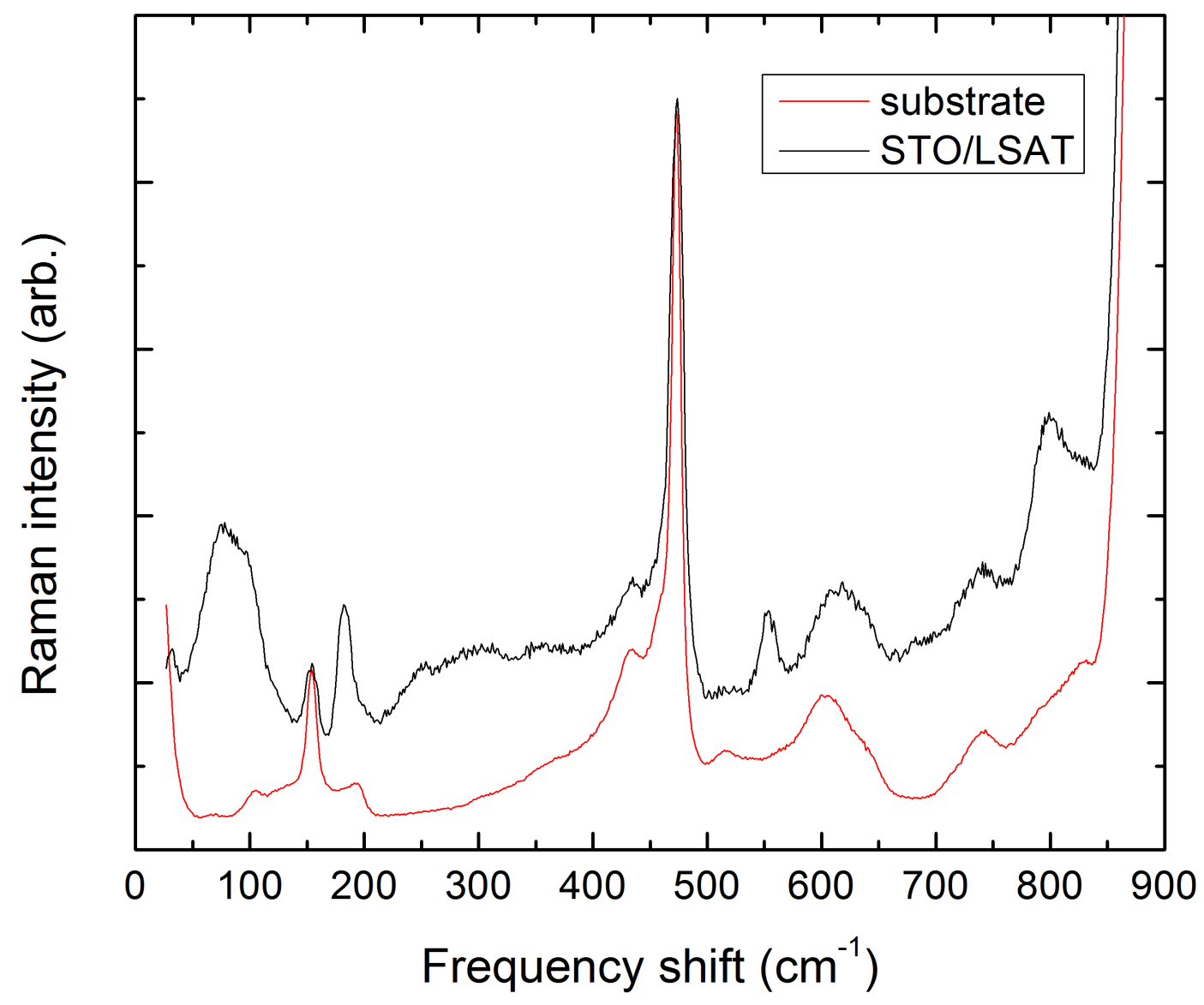

Figure 6.27: Raman spectra of sample STO006 - $105 \mathrm{~nm}$ of STO grown on LSAT, and of the LSAT substrate by itself. The spectra were both taken at $8 \mathrm{~K}$. 
Fig. 6.28 shows temperature dependence of the $\mathrm{TO}_{2}$ polar hard mode and the $100 \mathrm{~cm}^{-1}$ feature. The $\mathrm{TO}_{2}$ feature shows the familiar drop in intensity as temperature is raised, the point of most rapid decrease around $70 \mathrm{~K}$, and finishing the decrease at around 80-100 K. As compared to STO267, this S-shaped curve is centred considerably lower in temperature, and is much less broad. In this figure, note the different behaviour of the $77 \mathrm{~cm}^{-1}$ peak in this sample compared with the $93 \mathrm{~cm}^{-1}$ peak in STO267 - it first lowers, and then rises in frequency as temperature is increased, with the changeover point occurring at about $80 \mathrm{~K}$. In STO/LAO this peak just monotonically decreased in frequency. The temperature at which the frequency-change reverses direction is near to the temperature at which the rapid drop in intensity of the polar hard modes finishes. As with sample STO267, it is possible that this is the ferroelectric soft mode. Many effects cause this mode to harden compared to the single crystal STO case, making this a plausible assignment. The softening followed by hardening displayed here by this mode is very similar, for example, to the behaviour of $\mathrm{TO}_{1}$ reported by [44] from IR studies of thick, non-epitaxial films of STO.

\subsubsection{Sample STO005: $140 \mathrm{~nm}$ STO/LSAT measured in helium cryo- stat}

Film 005 is a thicker film grown in the exact same way as film 006 . The only difference was a longer deposition time. The thickness of this film is $140 \mathrm{~nm}$ as measured by Dektak profilometer. Fig 6.29 shows Raman spectra of the $140 \mathrm{~nm}$ layer (upper) and the $105 \mathrm{~nm}$ layer (lower) at five different temperatures, from $50 \mathrm{~K}$ to $100 \mathrm{~K}$, over the range where the polar modes disappear. Comparing the two side by side we see a few differences. First of all, the substrate features are stronger in the thinner layer. This is not surprising, as there will be less absorption of the optical field before the wave hits the substrate (and less absorption of the scattered field as it escapes the sample). Next, the thicker layer has a stronger signal from the second order features in STO. This can be seen best in the band between 250 and $400 \mathrm{~cm}^{-1}$. However, the thinner layer has stronger signal from the polar modes than the thick one. These two phenomena could be explained by considering that the polar regions of the crystal are significantly unevenly distributed through the film, with a larger concentration towards the film/substrate interface and smaller 


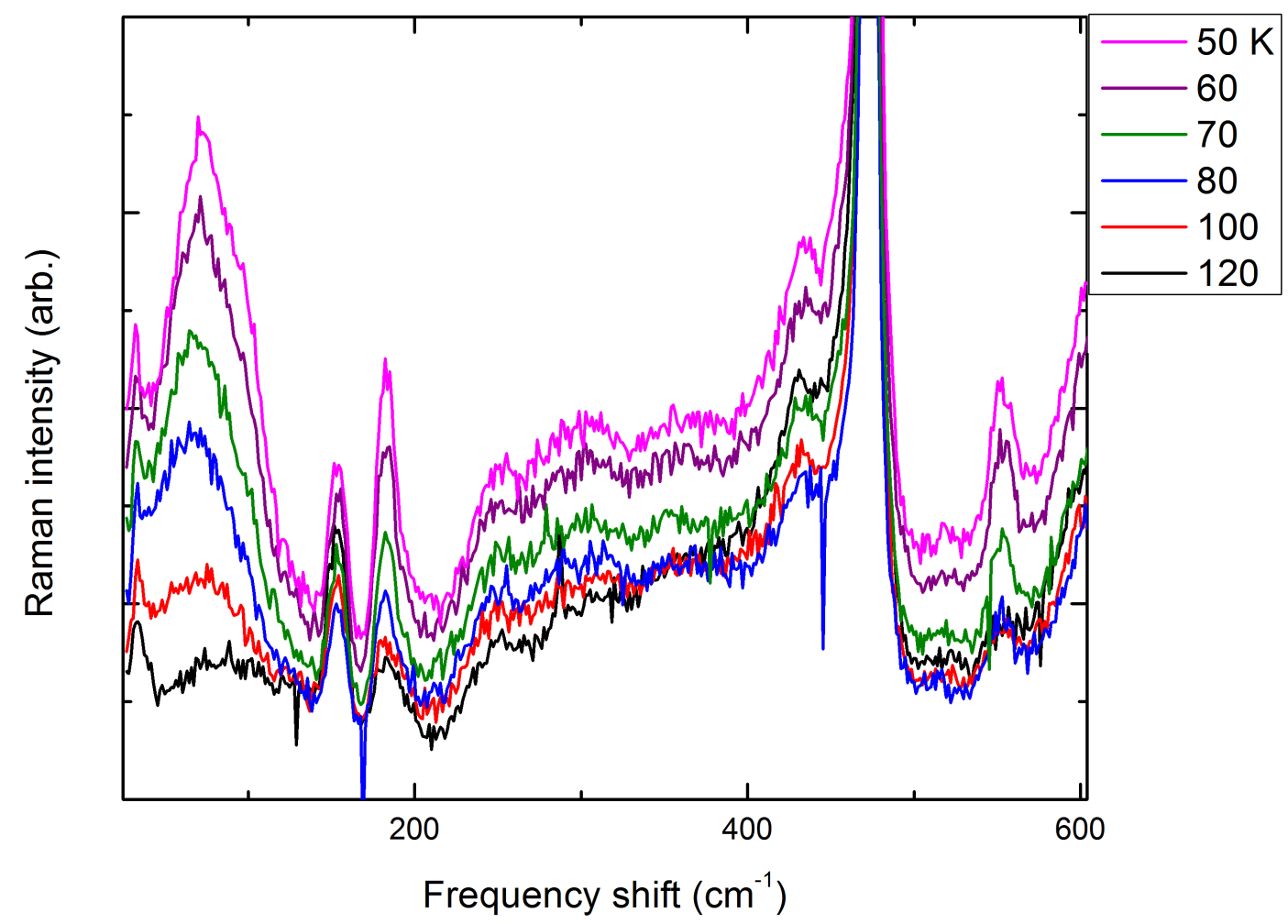

Figure 6.28: Temperature dependent Raman spectra of sample STO006. The spectra have been vertically offset for ease of viewing. The emergence of the $\mathrm{TO}_{2}$ and $\mathrm{TO}_{4}$ modes together with the $100 \mathrm{~cm}^{-1}$ feature can be seen here. 
140nm STO/LSAT film (upper, brighter) vs 105nm STO/LSAT film (lower, darker)

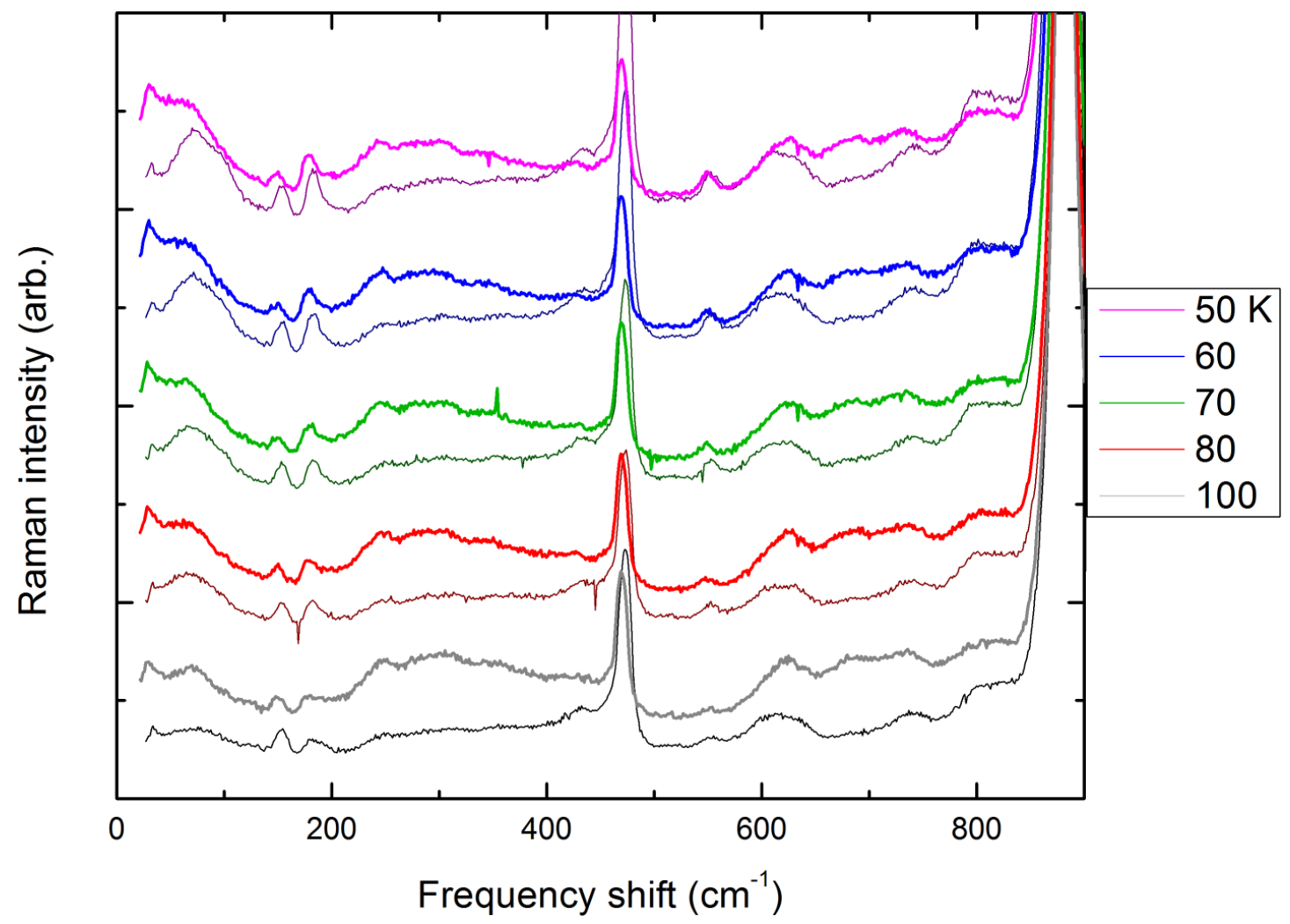

Figure 6.29: Comparison of the Raman spectra of $105 \mathrm{~nm}$ STO grown on LSAT to $140 \mathrm{~nm}$ STO grown on LAO. Spectra are shown at five different temperatures across the range where first order scattering emerges, and each temperature is vertically offset for ease of viewing. Lighter colours are the thicker layer, darker colours are the thinner layer. The temperature dependence of the $\mathrm{TO}_{2}$ and $\mathrm{TO}_{4}$ modes is very similar between the two samples.

concentration towards the film/air interface. Both the level of lattice strain, and the concentration of dislocation defects will be higher nearer to the film/substrate interface, and both of these elements have been shown to induce polar distortions in STO crystals. The thicker film, therefore, will have a larger scattering volume which is non-polar and shows only second-order features. Furthermore, this greater thickness of non-polar STO will absorb more light before the wave reaches the polar regions, explaining the reduced polar mode intensity.

The final important difference is the existence of a clear shoulder of the $70 \mathrm{~cm}^{-1}$ peak at $99 \mathrm{~cm}^{-1}$ in the thinner film, but not in the thicker film. This shoulder 
emerges at around $70 \mathrm{~K}$, and persists down to $10 \mathrm{~K}$. It could be that this line is the result of symmetry breaking, splitting redundant components of a vibrational mode. Such a splitting, if that is really the explanation for the additional shoulder would imply that the material undergoes a further structural phase transition. It is also possible that the double peak feature could be caused by two peaks of similar frequency from different regions of the film - a high strain and low strain region, for example. Looking closely at the thicker film spectra, one can see weak 'bump' features at the same frequency as the shoulder. These features could be the same, it is difficult to tell. Despite the subtle differences present, the two films have almost identical temperature dependence in the polar TO and LO mode intensities. The polar mode temperature dependence suggests that the additional thickness of film in the thicker sample contributes nothing to the growth of polar regions; and hence also contribute nothing to the degree of strain, the displacement concentration, or to the concentration of any other kinds of defects that cause changes to the temperature of ferroelectric transition. The results overall suggest that the vast majority of the ferroelectricity in STO strained thin films occurs within the first $100 \mathrm{~nm}$ of the film.

Fig. 6.30 shows the normalised fitted height parameter of the $\mathrm{TO}_{2}$ mode for the $105 \mathrm{~nm}$ and $140 \mathrm{~nm}$ STO/LSAT layers. Note the very similar behaviour between the two samples - both show a residual tail above $100 \mathrm{~K}$, and a rapid rise in intensity starting at about $80 \mathrm{~K}$. The noise in this data makes it difficult to tell if a saturation of peak intensity is reached below $20 \mathrm{~K}$, or if a pattern of continued increase in intensity is shown at the lowest temperatures. Assuming that a saturation does show at very low $\mathrm{T}$, the S-shaped curve made by these samples is considerably thinner than that of the STO layers on LAO - about $80 \mathrm{~K}$ compared with about $150 \mathrm{~K}$. It is comparable in width to the behaviour of the polar $\mathrm{LO}_{4}$ mode in NGO.

\subsubsection{Sample STO004: $60 \mathrm{~nm}$ STO/LSAT measured in helium cryostat}

Fig. 6.31 shows this sample compared with the substrate (scaled to fit the same graph) at $10 \mathrm{~K}$. This sample does not at first glance appear different from the substrate, except that the signal is much weaker. This is most likely a result of absorption of the UV excitation laser by the film. It could also be a result 


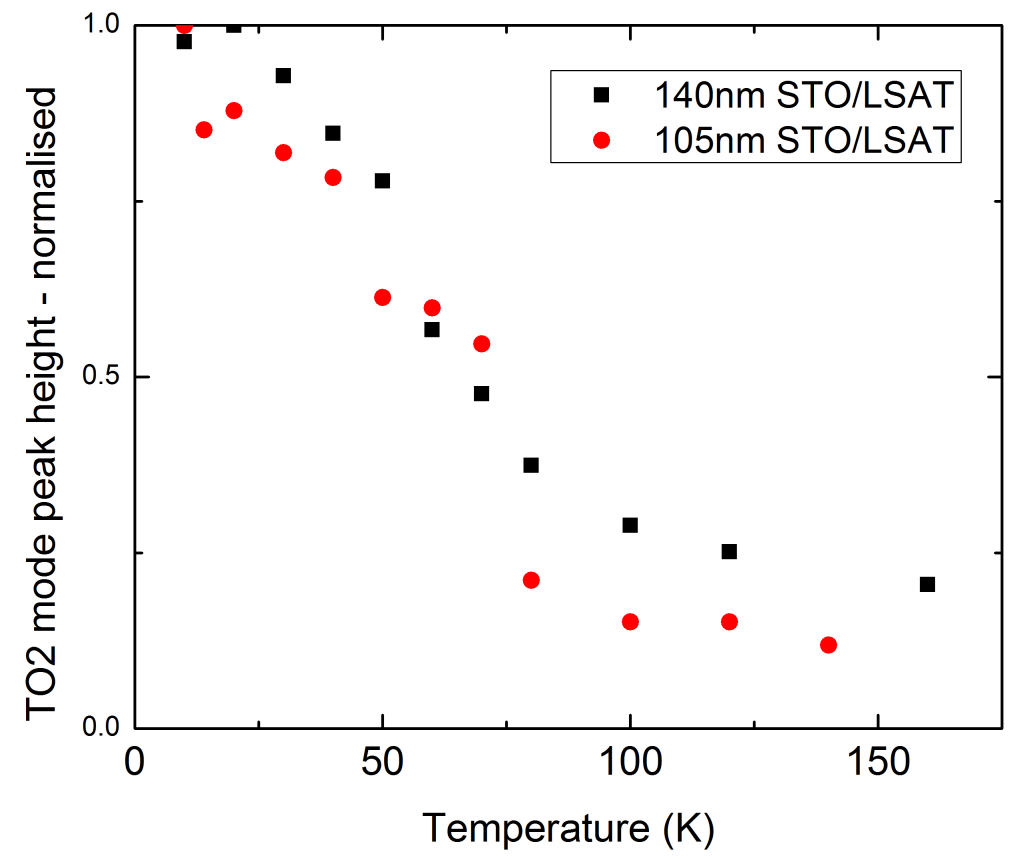

Figure 6.30: Temperature dependence of the first-order STO modes in $105 \mathrm{~nm}$ and $140 \mathrm{~nm}$ of STO grown on LSAT.

of different reflectivities from the STO layer and the LSAT substrate. In the helium cryostat, the excitation light incident on the sample does not enter normal to the surface, but at an angle. Any reflectance is therefore likely to be higher, and differences in reflectance shown more clearly. For sure, there is no peak at $550 \mathrm{~cm}^{-1}$ to attribute to the $\mathrm{TO}_{4}$ peak visible above noise in this sample. However, the peak at $183 \mathrm{~cm}^{-1}$ is higher compared to the $150 \mathrm{~cm}^{-1}$ peak in sample STO004 than in the substrate. It seems likely that this is the $\mathrm{TO}_{2}$ peak, which is usually the strongest of the polar modes in literature. The absence of the other first order modes is still puzzling, however.

Fig. 6.32 shows the temperature dependence of the $183 \mathrm{~cm}^{-1}$ peak as temperature is raised, and sure enough we see a rapid change in intensity. In this sample, a saturation in polar mode intensity is obvious, compared with sample STO005. This gives more confidence that the saturation suggested by the data on the thicker layers is real. The peaks were fitted wth Fano function lines, and the height parameters plotted with respect to temperature. Fig. 6.33 shows this parameter, along with the height parameter of $\mathrm{TO}_{2}$ (scaled to a substrate line) in samples 005 and 006 as well. Like the thicker samples, the S-shaped curve is 


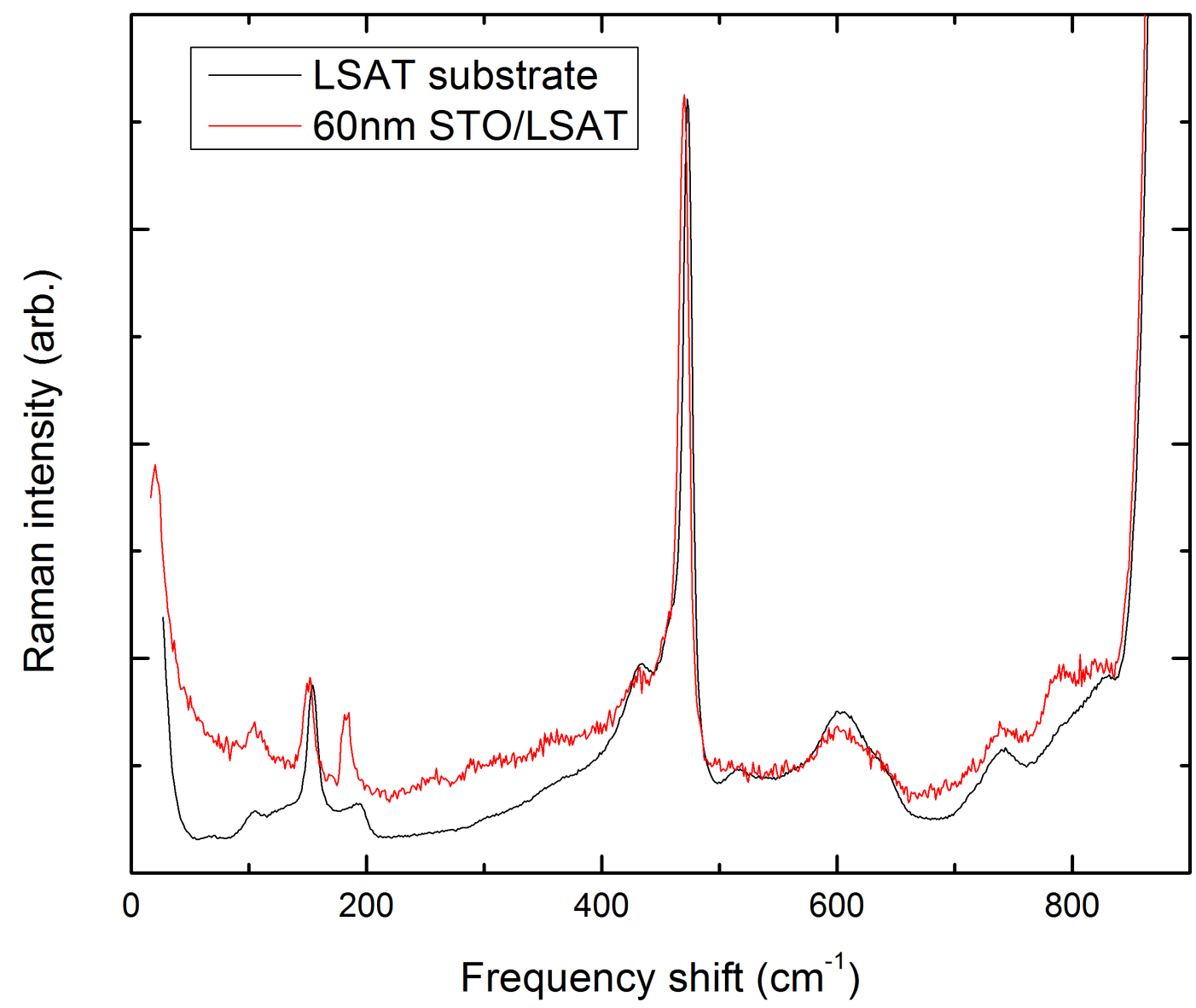

Figure 6.31: Raman spectra of sample STO004, a thinner film of STO grown on LSAT compared with the substrate. Only the $\mathrm{TO}_{2}$ mode is clearly seen above the substrate lines in this sample. 


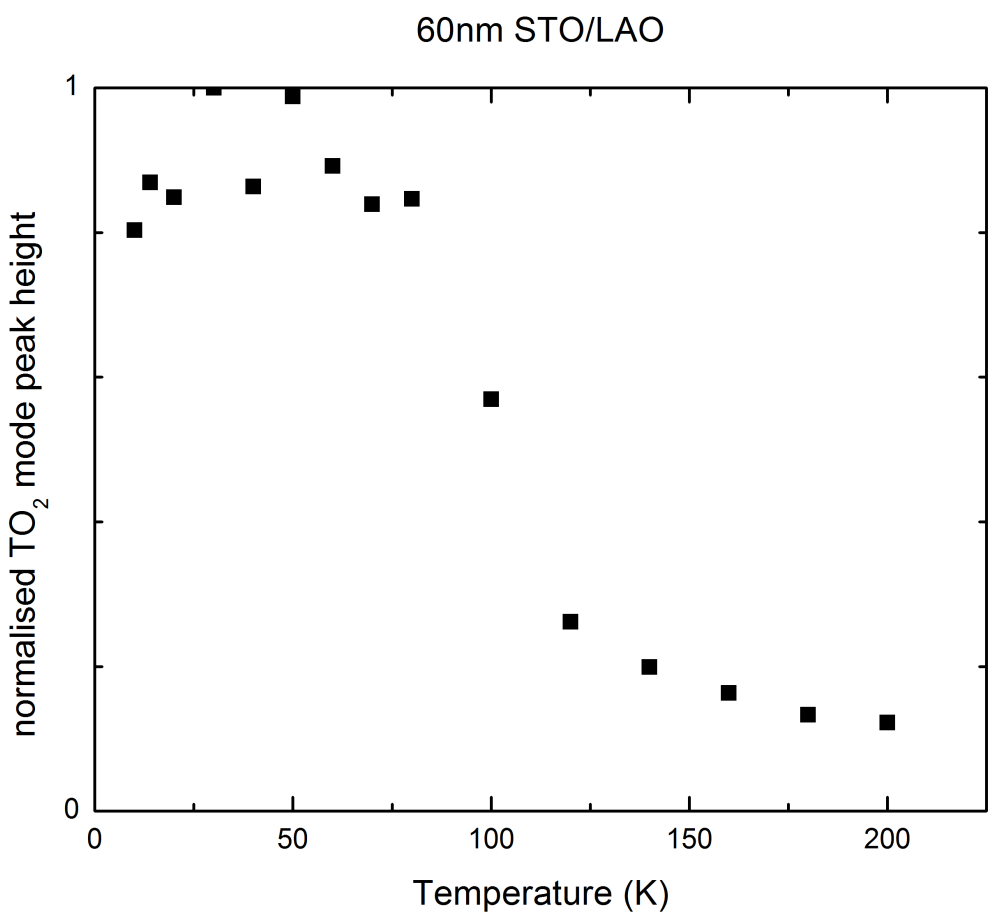

Figure 6.32: Temperature dependence of the $\mathrm{TO}_{2}$ mode intensity in sample STO004. The transition is relatively sharp and at a relatively high temperature compared to the thicker STO/LSAT samples.

narrow compared to STO/LAO. However, this thinner 004 film has its point of most rapid decrease at $100 \mathrm{~K}, 30 \mathrm{~K}$ higher than in the thicker samples 005 and 006. When we look at the temperature dependences side by side, we get a pattern very similar to that shown by the thinner and the thicker of the STO/LAO films, reinforcing the result that the polar state in these crystals is more stable in thinner films than in thick ones (although the similarity of the $105 \mathrm{~nm}$ and $140 \mathrm{~nm}$ films shows us that there is a limit to this effect in the high-thickness region).

\subsubsection{Summary of results - STO on LSAT}

Sample STO004 has thickness of $60 \mathrm{~nm}$ as measured by dektak profilometer. The first order $\mathrm{TO}_{2}$ mode is clearly seen in the spectra, and its temperature dependence shows an S-shaped curve with width of about $80 \mathrm{~K}$, with saturation occurring at $70 \mathrm{~K}$.

Sample STO006 has thickness of $105 \mathrm{~nm}$ as measured by dektak profilometer. 


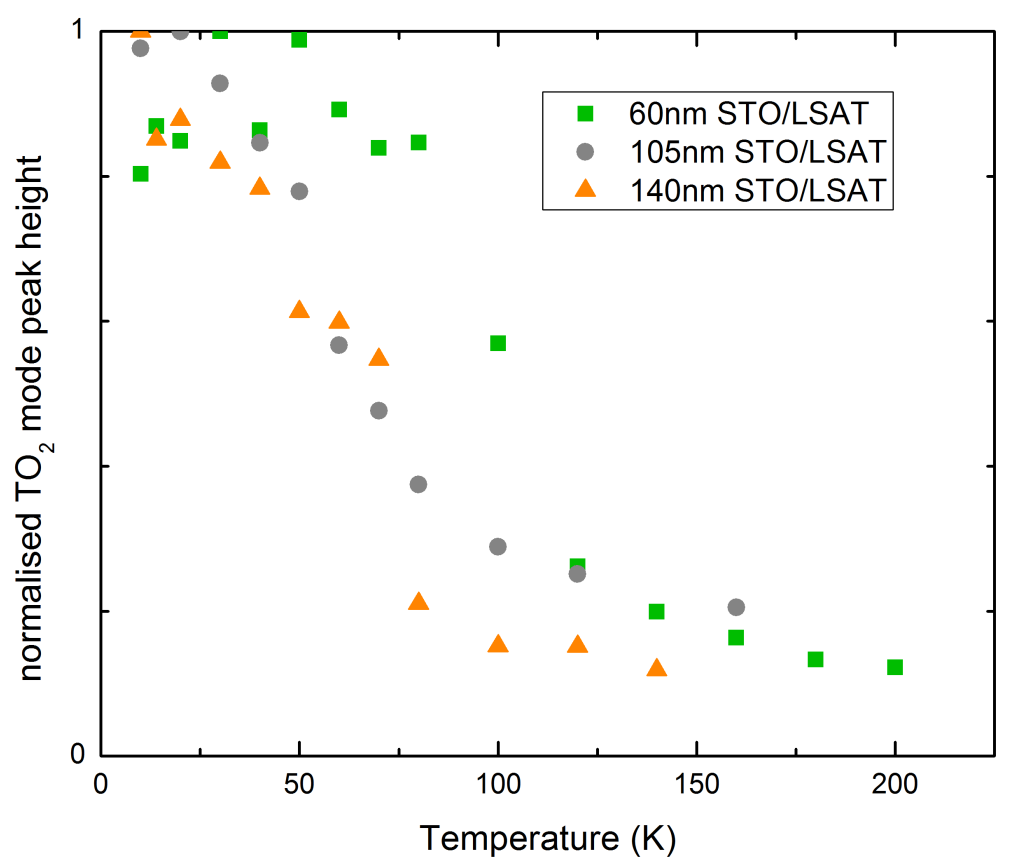

Figure 6.33: Temperature dependence of the $\mathrm{TO}_{2}$ mode intensity in all three STO/LSAT samples. The similarity of the two thicker films, and the contrast with the thinnest film can be seen.

The first order $\mathrm{TO}_{2}$ temperature dependence shows an S-shaped curve with width of about $80 \mathrm{~K}$, with saturation occurring at $30 \mathrm{~K}$. This mode additionally shows a feature with two peaks at $100 \mathrm{~cm}^{-1}$.

Sample STO005 has thickness of $140 \mathrm{~nm}$ as measured by dektak profilometer. The first order $\mathrm{TO}_{2}$ temperature dependence shows an S-shaped curve with width of about $80 \mathrm{~K}$, with saturation occurring at $30 \mathrm{~K}$. This mode also shows a feature at $100 \mathrm{~cm}^{-1}$, only one peak is seen and it is stronger than in sample STO006.

\subsection{XRD measurements}

In order to more fully understand the nature of the strain in our STO samples, XRD measurements were performed. The XRD results reported here are preliminary measurements which measure only the out-of-plane lattice constants, which is not the direction in which strain is applied nor in which the ferroelectric polarisation of unit cells is predicted to occur in STO. Measurements of the in-plane 
lattice constants are being undertaken, but are not available at the time of writing.

The contraction of the in-plane lattice constants also creates an expansion in the out-of-plane lattice constant, so what we can measure by these easier experiments still gives us some information about the strain in our samples.

Fig. 6.34 shows XRD 2 $\theta / \theta$ measurements of each of the thin films of STO/LSAT, showing out-of-plane lattice constants compared to a pure STO substrate. The spectra are plotted with intensity on a logarithmic scale, and are all taken at room temperature. The highest peak at $\theta=46.95^{\circ}$, which is present in the films, is that of the LSAT substrate. The next highest peak at $46.5^{\circ}$ is the relaxed position of the STO out-of plane lattice constant. The spectrum of the $60 \mathrm{~nm}$ film shows an STO peak at $46.10^{\circ}$, and it also shows interference fringes indicating a very small thickness. The thickness of the film can be calculated using these fringes, and it confirms the dektak profilometer measurement. The $105 \mathrm{~nm}$ film, the next thickest has a single peak at $46.2^{\circ}$, although it also displays a shoulder on the high angle side. The $140 \mathrm{~nm}$ film, which is the thickest, displays two peaks: one in the same place as the $105 \mathrm{~nm}$ film and another at higher angle $46.4^{\circ}$ - almost the same $2 \theta$ value as fully relaxed STO.

So what we observe with increasing thickness is a relaxation of lattice constant towards the normal value. The thinnest films show a lattice constant highly distorted from the relaxed unit cell length. As thickness increases, the distortion relaxes until it reaches the point at approximately $46.2^{\circ}$. After this, a second phase begins to form, with lattice constant nearly the same as the relaxation value. However, this forms in a mixed phase, with volumes in the $46.2^{\circ}$ distorted phase also present in the same sample.

This pattern follows almost exactly the pattern implied by the Raman spectra, in which we see the polar mode intensity saturating at the highest temperature for our thinnest film, and at a lower temperature for the thicker films. The thicker films both show Raman mode saturation at the same temperature, and I propose that this signal is coming from the more highly strained layer in each, which XRD shows to be the same in both samples. The additional thickness of relaxed STO on the thickest film explains why the polar modes are weaker in the thickest film - greater absorption by a non-scattering layer. The XRD reveals new information 
about the strained and unstrained layers in thicker films though - where before we assumed the relaxation of strain across the thickness of a single sample to be continuous, the XRD shows a quite distinct separation from high-strain to lowstrain regions in the films, suggesting a very small region over which the strain in the sample changes rapidly at a point part way through the thickness of the film.

We can confidently say that thin films of STO/LSAT grown with thicknesses below the relaxation threshold of close to $100 \mathrm{~nm}$ have strain that is dependent on thickness. So - in the thinner films, up to about $100 \mathrm{~nm}$, the ferroelectric layer is grown. Changes to the thickness of the film within this range of thickness have thickness dependent strain, and can still create material differences in the ferroelectric properties of the thin film. Once this soft threshold is reached, further STO is grown in a dielectric layer, that does not any longer increase the volume of polar STO.

\section{$6.6100 \mathrm{~cm}^{-1}$ feature}

Now armed with all of the spectra from all of our samples, I can tackle the problem of the $70-100 \mathrm{~cm}^{-1}$ feature present in many of the spectra. A feature of this kind is present in both of the STO/LAO films; and in the thicker two STO/LSAT films but not the thinnest STO/LSAT film; and it is not seen in the STO/NGO film, although there is a feature in the substrate at this temperature which would effectively mask it if present in the film, so this absence is only a tentative assignment.

The most obvious candidate for a mode giving these peaks in the Raman spectra is the ferroelectric soft mode $\left(\mathrm{TO}_{1}\right)$. The presence of this mode at such an elevated frequency in the literature is always associated with a non-ferroelectric phase in STO. However, in the STO/LSAT samples, the thicker of the STO/LSAT films do show the $100 \mathrm{~cm}^{-1}$ feature, and I am reasonably confident of ferroelectricity based on the similarity of the polar hard mode temperature dependence to that of STO/NGO.

An important observation to make of this $100 \mathrm{~cm}^{-1}$ feature is that it is stronger in the thicker films than the thinner films on both substrates. The thicker films 


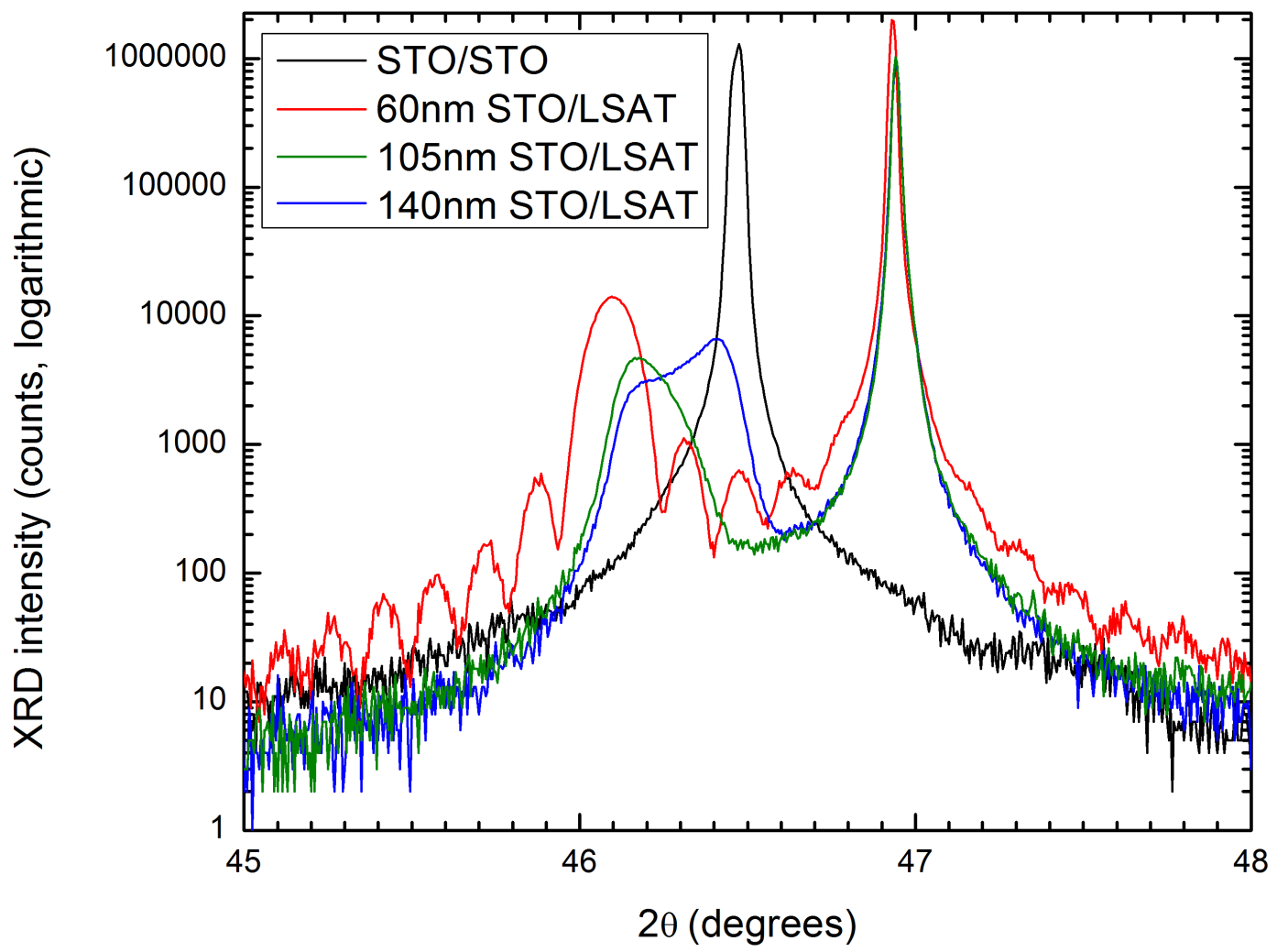

Figure 6.34: Room temperature XRD spectra of STO thin films grown to various thicknesses on LSAT. A spectrum of STO/STO is provided for reference. The out-of plane lattice constant is measured in these spectra, and the variation of the lattice constant with film thickness cna be seen. There is clearly a correlation between strain and thickness, and there is also clearly a two-phase growth mode in the thickest sample. 
show higher intensity of this feature, both in comparison to the substrate modes and in comparison to the other STO modes. Taken together with the XRD measurements, this makes me confident that the feature is being generated mostly in the low-strain region of the films. Take the STO/LSAT system for example, refering to Fig. 6.34. The thickest film has the strongest $100 \mathrm{~cm}^{-1}$ feature, and it has the greatest volume of film in the relaxed phase. The $105 \mathrm{~nm}$ film has a shoulder on the relaxed strain side, showing evidence of some volume of relaxed STO, and it shows the $100 \mathrm{~cm}^{-1}$ at weaker intensity than the thick film. The thinnest film shows no sign of either the $100 \mathrm{~cm}^{-1}$ feature, or a relaxed strain phase. This pattern is repeated in the $\mathrm{STO} / \mathrm{LAO}$ samples, the thickest film has much stronger intensity of the feature.

Considering this evidence, and also considering the mixed phase growth of highthickness strained STO samples discovered in the XRD measurements, I suggest that the $100 \mathrm{~cm}^{-1}$ feature is the ferroelectric soft mode, in regions of STO under low levels of strain. Their presence in the spectra at greatly hardened frequencies therefore does not imply anything about the highly strained volume of the film. The most obvious candidate to explain the soft-mode hardening in the low-strain regions is lattice defects such as dislocations existing between the high-strain and low-strain layers. Recall the distinct two-phase growth shown in the XRD measurements. This suggests a thin interface between high- and low-strain layers, and the dislocations defects breaking epitaxy at this interface would be similar to the 'dead layer' examined in many STO thin films which have been attributed with causing low ferroeletric and dielectric properties and hardening the soft mode (refer to section 2.3.4).

\subsection{Strain - phase transition relationship}

In each of our STO thin film samples we now have some measure of a phase transition temperature from the AFD tetragonal phase to a different low temperature phase. In sample STO264 grown on NGO, we have some proof that the new phase is of lower symmetry in the splitting of the AFD soft mode. In this sample at least, it seems very likely that the new phase is the orthorhombic, ferroelectric phase seen in other STO samples in the literature. There is no evidence to sug- 
gest that the low temperature phase of the samples grown on other substrates is different to the sample grown on NGO. The nature of this phase transition, in all of the samples we have measured is a slow-with-temperature transition from one phase to the next, most likely through a polar micro-region percolation as has been reported for other STO systems. We expect though that the distribution of the polar regions will not be disordered as in other systems, but be growing from the substrate-film interface up, as strain is highest close to the boundary.

Using the temperature dependence of the polar STO modes in each of the samples, a value for $\mathrm{T}_{C}$ was calculated. The value was designated as the point at which a tangent to the steepest part of the curve intercepts with the saturated low intensity value. This is illustrated in Fig. 6.35. On sample STO264, this calculated value for $\mathrm{T}_{C}$ corresponds very well to the temperature where the splitting of the AFD mode is observed. A single temperature is not sufficient to describe the complex process of the transformation from one phase to another in these films, which takes place over a long range of temperatures. During that range, either the order parameter, or the volume of the film in the low temperature phase or both must be changing. So I include a second parameter, a $\mathrm{T}_{C}$ width which describes the temperature range over which the polar modes are changing in intensity. The figure is the range between $5 \%$ and $95 \%$ of the saturation intensity, rounded to the nearest $10 \mathrm{~K}$.

The XRD measurements displayed in this thesis showed that, at least for the samples grown on LSAT, large amounts of strain relaxation (away from the lattice mismatch value) were correlated with strain-inhomogeneity in the film. This inhomogeneity casts into doubt the usefulness of the strain measurements presented for the samples grown on LAO in section 3.1.3. As the XRD only measures a single average value, and the measured values are in this case strongly relaxed from the lattice mismatch value, it is highly likely that the figures are way off the strain in the region of the film which gives us the polar Raman signal. In other words, I think that the level of strain in the polar region of the films is very different from the measured, average strain. This is different for the NGO film, which showed a very small level of relaxation from the lattice mismatch value. The strain is likely to be much more homogeneous throughout the film in this case, and the average strain therefore represents much more closely the strain of the polar volume. In the table and phase diagram presented below, the strain quoted for the STO/LAO 
100nm STO/LAO

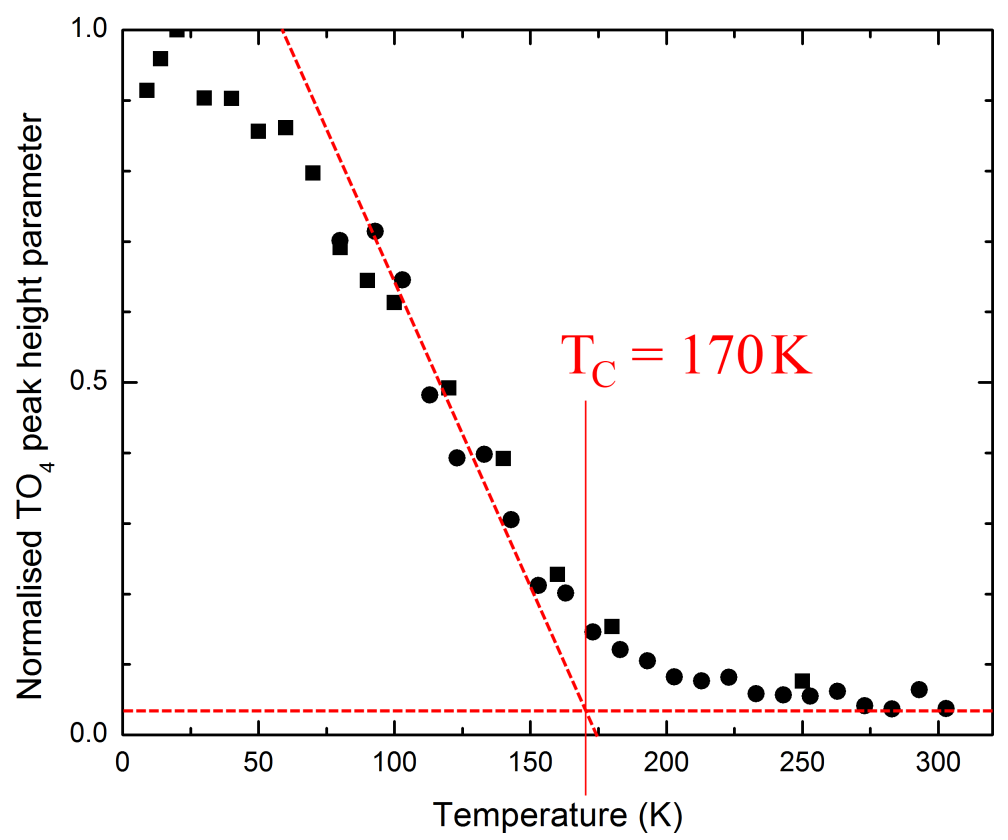

Figure 6.35: Example of how $\mathrm{T}_{C}$ for the STO thin films was calculated. A tangent to the polar amplitude vs. T curve was drawn at the steepest point, and a horizontal line was drawn at the low amplitude saturation point (dotted red lines). The intercept of these two lines was taken as the phase transition temperature. 
and STO/LSAT samples are estimated based on the lattice mismatch and the thickness of the film. For the LSAT films, I have some confidence in the estimates because they are also informed by the out-of-plane XRD measurements. For the LAO samples, I am far less confident of the accuracy of the values. For the sake of displaying the data, I have chosen values, but be aware that there is a huge uncertainty in these values - $\pm 1 \%$ would not be unreasonable. The phase diagram made is found in Fig. 6.36. It shows the phases predicted by the theory presented in [1], the $\mathrm{T}_{C}$ s calculated from our Raman data, and the $\mathrm{T}_{C}$ width.

\begin{tabular}{llllll}
\hline Sample & Subs & Est. in-plane strain & Thickness & $\mathrm{T}_{C}$ & $\mathrm{~T}_{C}$ width \\
\hline STO016 & STO & $0 \%$ & $50 \mathrm{~nm}$ & none & none \\
\hline STO263 & LAO & $-2.5 \pm 1 \%$ & $30 \mathrm{~nm}$ & $210 \pm 15 \mathrm{~K}$ & $150 \mathrm{~K}$ \\
\hline STO267 & LAO & $-1.5 \pm 1 \%$ & $100 \mathrm{~nm}$ & $170 \pm 15 \mathrm{~K}$ & $150 \mathrm{~K}$ \\
\hline STO264 & NGO & $-1.3 \pm 0.1 \%$ & $50 \mathrm{~nm}$ & $160 \pm 10 \mathrm{~K}$ & $80 \mathrm{~K}$ \\
\hline STO004 & LSAT & $-0.95 \pm 0.1 \%$ & $60 \mathrm{~nm}$ & $130 \pm 15 \mathrm{~K}$ & $100 \mathrm{~K}$ \\
\hline STO005 & LSAT & $-0.8 \pm 0.2 \%$ & $140 \mathrm{~nm}$ & $90 \pm 15 \mathrm{~K}$ & $100 \mathrm{~K}$ \\
\hline STO006 & LSAT & $-0.8 \pm 0.2 \%$ & $105 \mathrm{~nm}$ & $90 \pm 15 \mathrm{~K}$ & $100 \mathrm{~K}$ \\
\hline \hline
\end{tabular}

The data show a relationship between biaxial strain and phase transition temperature which falls nicely into the predicted range, albeit with large uncertainties in the strain of some samples.

\subsection{STO strained thin films - conclusions}

UV Raman spectroscopy measurements were carried out on samples of STO thin films, epitaxially grown on lattice-mismatched substrates to induce biaxial strain. The UV method was found to enhance the film signal relative to the substrate signal enough such that spectral features of STO could be seen. Spectral features of polar STO were found in all of the strained samples, showing a variation in range of ferroelectric transition temperatures which was dependent on the degree of strain. The relationship between strain and transition temperature shown by the Raman spectra fits well to theoretical calculations found in the literature. 


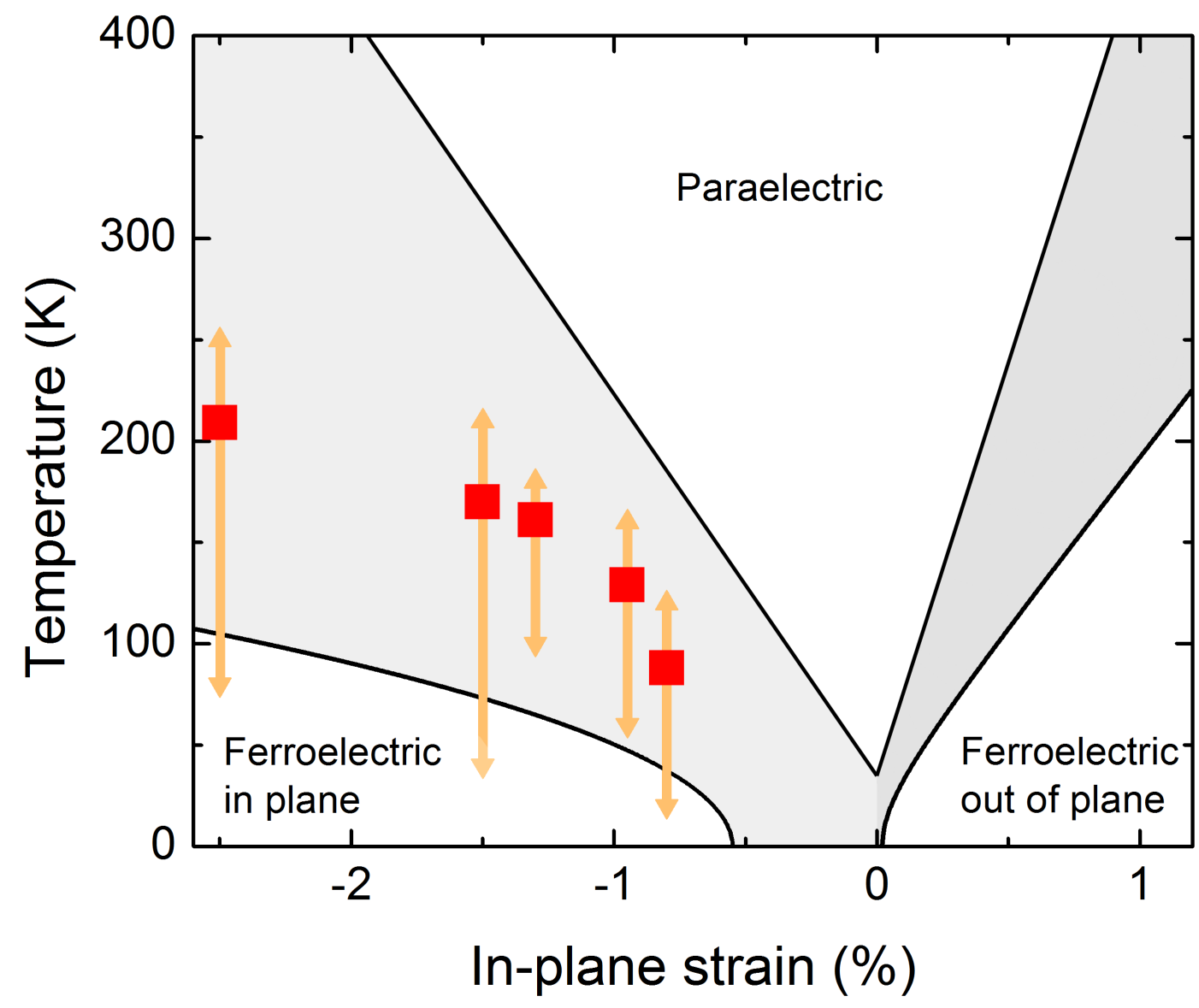

Figure 6.36: Phase diagram of biaxially strained STO. The phases marked and the grey shaded regions between them are copied from the theory presented in [1]. Red squares show calculated phase transition temperatures as found by the Raman spectroscopy measurements in this thesis. Orange bars show the temperature range over which the polar Raman features are changing in intensity. This may indicate a growth of polar volume, an increase in polar order parameter or a combination of the two. 
The phase transition is a slow-with teperature process which shows either a co-existence of phases over a long temperature range, or a slow increase in order parameter over that range, and an eventual maximum value of the parameter reached - or some combination of those two factors. The samples grown on LAO had the highest lattice mismatch in the study, and also had the greatest temperature range of phase transition. The other samples had comparable temperature ranges of transition, smaller than the LAO substrate samples.

XRD measurements at room temperature on STO/LSAT samples showed a relationship between sample thickness and strain. Up to a threshold thickness at near $100 \mathrm{~nm}$, thicker films on LSAT are under less strain. After the threshold is reached, there is a discontinuity, and a second phase begins growing. The second phase is almost perfectly relaxed, while the first phase remains under conditions of strain similar to the conditions at the threshold. 


\section{Chapter 7}

\section{Conclusions}

\subsection{BNT-BT - summary of results}

Temperature dependent Raman spectra were taken of samples of the mixed ceramic $\mathrm{Bi}_{0.5} \mathrm{Na}_{0.5} \mathrm{TiO}_{3}-\mathrm{BaTO}_{3}$, with barium substitution levels between $0 \%$ and $9 \%$. Using these spectra, we were able to show that a presumed structural phase transition in the system was false - the electrical step change across this boundary can be explained by a loss of long-range order in the material while the crystal structure is unchanged.

This study showed the usefulness of the Raman spectroscopy technique as a proxy for structural measurements. The great advantage of Raman spectroscopy in structural investigations is that it measures over very small length scales compared to bulk electrical and XRD measurements, and is thus a better probe of local structure.

This new information for the phase diagram of this system showed that the morphotropic phase boudary between ferroelectric rhombohedral and ferroelectric tetragonal phases did not terminate in a tricritical point at high temperatures, as was previously thought. The free energy landscape of the material is therefore less flat than was thought, which can explain the relatively low piezoelectric activity of BNT-BT compared to other systems. 
Poling the samples by placing the samples in strong electric fields could move the MPB by about $1 \%$ Ba substitution in favour of the rhombohedral phase, but does not otherwise alter the phase transitions in this material.

\subsection{BNT-BT - suggested further work}

The experiments performed on the BNT-BT system showed the importance of measuring the local structure in mixed ceramic systems such as BNT-BT. A logical next step in this research would be to re-examine other systems in which only bulk electrical measurements have been made using Raman spectroscopy to assign structural phase transitions.

It also helps direct lead-free ferroelectric research towards materials that contain not just a morphotropic phase boundary, but a triple point between two ferroelectric phases and a paraelectric phase in their phase diagrams - making certain of course that the electrical phase transitions correspond to structural ones as well.

\subsection{PT nanowires - summary of results}

Temperature dependent Raman spectra, and scanning electron microscope images were taken of unusual samples of lead titanate nanowires prepared by annealing PX phase (see section 3.1.2) wires into perovskite phase wires. The Raman spectra showed that there was an anomalously increased ferroelectric phase transition temperature in the PT nanowires.

The enhancement was found to be dependent on the diameter of the nanowires, as measured by SEM imaging. The $\mathrm{T}_{C}$ peaked at a value of greater than $600{ }^{\circ} \mathrm{C}$, an enhancement of more than $100 \mathrm{~K}$. The greatest enhancement was found in nanowires of $125 \mathrm{~nm}$ diameter. In wires larger and smaller than $125 \mathrm{~nm}$, the enhancement dropped away.

TEM images were taken of these samples by another group, and revealed an 
enhcancement in the tetragonality of the wires as well. Like the transition temperature enhancement, this was dependent on wire diameter, and peaked in wires of $115 \mathrm{~nm}$.

The TEM also revealed the presence of $\sim 10 \mathrm{~nm}$ voids in the wires. The concentration of these voids, like the $\mathrm{T}_{C}$ enhancement and the tetragonality enhancement was found to be dependent on the wire diameter. It increased up to diameters of $110 \mathrm{~nm}$, and in wires larger than this, cracks were seen.

We suggest that the nanoscopic voids formed in the wires are causing the enhancement in the other order parameters by exerting tensile strain in the wires, and we further suggest that the voids are formed due to the annealing process where they change from the less dense PX phase to the more dense perovskite PT phase.

\subsection{PT nanowires - suggested further work}

The discovery of this enhancement in parameters caused by a transformation from a less dense phase to a more dense phase is surprising and exciting. The next step in this research would be to try and find out how specific to lead titanate this effect is.

It would be interesting to try to find any other ferroelectric materials in which a similar transformation can be made. The material would ideally need to have a second accessible phase, less-dense than the ferroelectric phase, and which forms nanostructures with length scales on the order of $100 \mathrm{~nm}$. Particularly if some leadfree material could have their transition temperatures enhanced by this method, then that would be a great benefit to the ferroelectrics industry.

Another approach might be to engineer the growth of lead titanate nanowires in the PX phase. It would be valuable to try to find conditions to make wires of particular diameter or length, and to improve the homogeneity of the wires formed, either through some growth or sorting method.

Measurements of the piezoelectric constants, dielectric permittivity, and ferro- 
electric poling loops would also be beneficial in this system so that we can see how the other parameters of the wires are affected by the tensile strain.

One other open question in these systems is the change in morphology of the smallest nanowires at high temperatures. Our Raman spectroscopy and SEM results suggest that wires below $100 \mathrm{~nm}$ in diameter are not stable, having changed shape and migrated across the substrate, attaching to other larger PT nanowires. We were not able to precisely ascertain what led to the change in morphology, so further study in this area may be interesting.

\subsection{STO thin films - summary of results}

Strontium titanate thin films were epitaxially grown by pulsed laser deposition onto a variety of lattice-mismatched substrates in order to create biaxial strain in the thin films. The thin films are measured by ultraviolet Raman spectroscopy and $\mathrm{x}$ ray diffraction to investigate their structural phase diagrams, and the implications for strain-induced ferroelectricity.

The UV Raman spectroscopy method was able to successfully enhance signals from the films and supress signals from the substrates, and is shown to be a useful technique to measure thin films.

The Raman spectra we took showed signs of a phase transition in all of the strained samples. In one of the samples, we were able to see additional features in the Raman spectra indicating that this is the ferroelectric phase transition from tetragonal to orthorhombic symmetry of STO. Intense substrate lines prevented us from seeing these features in all the other samples. There was no sign of this transition in the unstrained film.

Signs of the other major phase transition in STO, the anti-ferrodistortive phase transition were almost always absent from the films. However, where they were present they indicated a significantly raised AFD phase transition temperature in the strained films.

XRD measurements of these samples were able to show the strain in thicker 
of the thin films was strongly inhomogeneous, with regions of strained STO and regions of relaxed STO present in the same sample. Thinner films would grow homogeneously, with strain inversely proportional to film thickness. At a threshold thickness, there is a discontinuity seen and subsequently the strain in the strained region is no longer thickness dependent, and relaxed STO grows on the top surface.

Signs of strain inhomogeneity are seen in the Raman spectra as well. The Raman signals of the phase transition do not appear suddenly. Rather, they slowly grow in intensity as temperature is lowered over a period of between $80 \mathrm{~K}$ and $170 \mathrm{~K}$, depending on the sample.

The ferroelectric phase transition temperatures for each sample were calculated from the temperature dependence of the polar mode amplitudes in the Raman spectra. In-plane strain was estimated from the thickness and lattice mismatch, and average strain measurement where available at time of writing. A relationship was found between $\mathrm{T}_{C}$ and biaxial strain which agreed with theoretical predictions made by other groups. It must be stated as a proviso though that the estimates of strain for some samples are not very good. The data could be much improved by a good measurement of the actual in-plane strain in the samples.

\subsection{STO thin films - suggested further work}

The results of this chapter are less complete than those in the other chapters. Many aspects of the results are not clear, so the most important further work to suggest is to answer the open questions.

First of all, measurements of the in-plane lattice strain in these samples would be greatly beneficial. An average lattice strain measurement is not sufficient due to the inhomogeneity in strain across the STO thin films. Measurements sensitive enough to see this varation in strain are required.

The NGO substrate was the best one for Raman measurements, as the substrate lines of that material did not overlap with the low-frequency soft modes. I suggest that it would be beneficial for more STO thin films be grown, of a variety of thicknesses on this material so that the most interesting Raman features can be 
seen in a variety of strain states. Once again though, it may prove difficult to grow STO epitaxially in a variety of thicknesses on NGO due to the non-square lattice.

It would also be valuable to make measurements of the electric permittivity and ferroelectric polarisation loops, if any, in these thin films. Right now the assignment of these materials as ferroelectric is made purely on the basis of the structural phase transitions, and as we have learned in BNT-BT the structural and electrical phase transitions are not necessarily equivalent.

Finally, another angle to take would be to measure other ferroelectric materials under lattice strain. The proof of concept in STO, that biaxial strain can raise structural transition temperatures has been shown well by these results. Guided by theory or by pressure-dependent structural measurements, it could be possible to find more materials that can be enhanced this way. This presents another way in which lead-free ferroelectric systems might be brought up to par with PZT such that they can be used in technology. 


\section{Appendix A}

\section{Paper in submission concerning PT}

\section{nanowires}

This is a recent copy (at time of printing) of an article in submission regarding the PT nanowires. The article goes much further in suggesting that tensile strain is the source of the anomalous enhancement of transition temperature in the PT nanowires than I do in this thesis and it draws on other measurements and theory that are not the contribution of the author of this thesis. This article is presented with its own images and references, which are independent of those in the thesis

\section{A.1 Nanopore-induced negative-pressure ferroelectric enhancement in freestanding $\mathrm{PbZrO}_{3}$ nanowires}

Jin Wang ${ }^{1}$, Ben Wylie-van Eerd ${ }^{2}$, Cosmin Sandu ${ }^{1}$, Marco Cantoni ${ }^{3}$, Pascale Gemeiner ${ }^{4}$, Brahim Dkhil ${ }^{4}$, Alexander Tagantsev ${ }^{1}$, Joe Trodahl ${ }^{2}$, Nava Setter ${ }^{1}$

${ }^{1}$ Ceramics Laboratory, Swiss Federal Institute of Technology (EPFL), 1015 Lausanne, Switzerland

${ }^{2}$ MacDiarmid Institute for Advanced Materials and Nanotechnology, Victoria University of Wellington, New Zealand 
${ }^{3}$ Interdisciplinary Centre for Electron Microscopy, Swiss Federal Institute of Technology (EPFL), 1015 Lausanne, Switzerland

${ }^{4}$ Laboratoire Structures, Propriétés et Modélisation des Solides, CNRS-UMR8580, Ecole Centrale Paris, Grande voie des vignes, 92290 Châtenay-Malabry, France.

\section{A.2 Abstract}

Ferroelectrics are ubiquitous in technology with a substantial interest in the extension of their operational temperature-range and exploitable properties. We report a strong enhancement of ferroelectric unit cell distortion $(c / a)$ and Curie temperature in freestanding $\mathrm{PbTiO}_{3}$ nanowires and present extensive evidence that the enhanced characteristics originate from negative pressure effects due to introduction of nano-pores in the material. The enhancements are maintained for years without degradation.

\section{A.3 Article}

Ferroelectrics find key application in fuel injection, high-precision actuation, ultrasonic detection and imaging, energy harvesting, nonvolatile information-storage, and capacitors in electronic circuitry ${ }^{i, i i, i i i}$. Parameters that define the usefulness of ferroelectrics include high piezoelectric/pyroelectric/dielectric coefficients, large spontaneous polarization, and extended working temperatures.

Strain influences strongly the ionic displacements that are at the core of ferroelectric properties. Thus there are recent exciting developments exploiting epitaxial films on lattice-mismatched substrates that impose modest tensile or compressive biaxial strain and enhance the propensity for the development of structural order parameters that characterize ferroelectrics ${ }^{i v, v, v i}$. Much larger hydrostatic stress is provided by diamond anvil cells, but its partner, hydrostatic tensile stress, is generally regarded as unachievable. Nonetheless there are interesting expectations for this negative pressure regime. The semi-empirical Landau-Ginsburg-Devonshire 
expansion predicts a doubling of the order parameter $(c / a-1)$ in $\mathrm{PbTiO}_{3}$ under negative pressures of a few GPa. A strong pressure dependence is confirmed in experiments under positive pressures in a diamond anvil cell ${ }^{\text {vii,viii }}$ in which the order parameter is strongly reduced. An ab initio treatment has predicted an even stronger and nonlinear enhancement under negative pressure of 4-6 GPa, signaling the development of a new structural phase ${ }^{i x}$. However, it is difficult to apply strain engineering to freestanding samples such as single crystals and ceramics that lack bonding to a clamping media. Here we report the observation of a strong enhancement of the unit cell distortion $(c / a)$ and Curie temperature $\left(\mathrm{T}_{C}\right)$ in freestanding nanoporous monocrystalline $\mathrm{PbTiO} 3(\mathrm{PT})$ nanowires, which is caused by a negative pressure effect.

Our wires were initially prepared in the body-centered tetragonal PX phase, with the same stoichiometry as perovskite PT but having an accicular crystalline structure of a lower density $\left(6.87 \mathrm{~g} / \mathrm{cm}^{3} \mathrm{vs} 7.97 \mathrm{~g} / \mathrm{cm}^{3}\right)^{x, x i}$. The perovksite nanowires, with a thickness ranging from $20 \mathrm{~nm}$ to $500 \mathrm{~nm}$ (Fig. 1a) were obtained by annealing the PX nanowires in air at about $540{ }^{\circ} \mathrm{C}$. Nearly-spherical pores with a typical diameter of $10 \mathrm{~nm}$ did not break their monocrytallinity (Fig.1c). Bending occurred in some of the converted perovskite nanowires and $90^{\circ}$ domains were frequently found, especially across the bent regions (Fig.1d).

The annealed nanowires show a tetragonal perovksite structure as confirmed by XRD measured on the ensamble (Fig. S1a) and by the Raman spectra of individual nanowires (Fig. S1b). The spontaneous polarization direction in the nanowires, the $\mathrm{c}$ axis of the tetragonal perovskite cell, did not adopt any specific orientation with respect to the geometry of the nanowires. The $c / a$ ratio of individual nanowires was investigated by high resolution transmission electron microscope (HR-TEM) for nanowires thinner than $120 \mathrm{~nm}$ and selected area electron diffraction (SAED) for thicker wires. A remarkable result is that in a small range of diameters near $110 \mathrm{~nm}$, there is an enormously enhanced tetragonality $(c / a-1)$ up to about 0.13 (Fig. 1e), approximately doubling the tetragonality from $(c / a-1)=0.065$ in bulk $\mathrm{PT}^{x i i}$. Both thinner or thicker wires exhibited c/a ratio similar to the bulk PT value, within the measurement uncertainty.

The strongly enhanced tetragonality suggests that the wires might show an enhanced ferroelectric instability ${ }^{x i i i}$. Thus we have investigated the ferroelectric- 

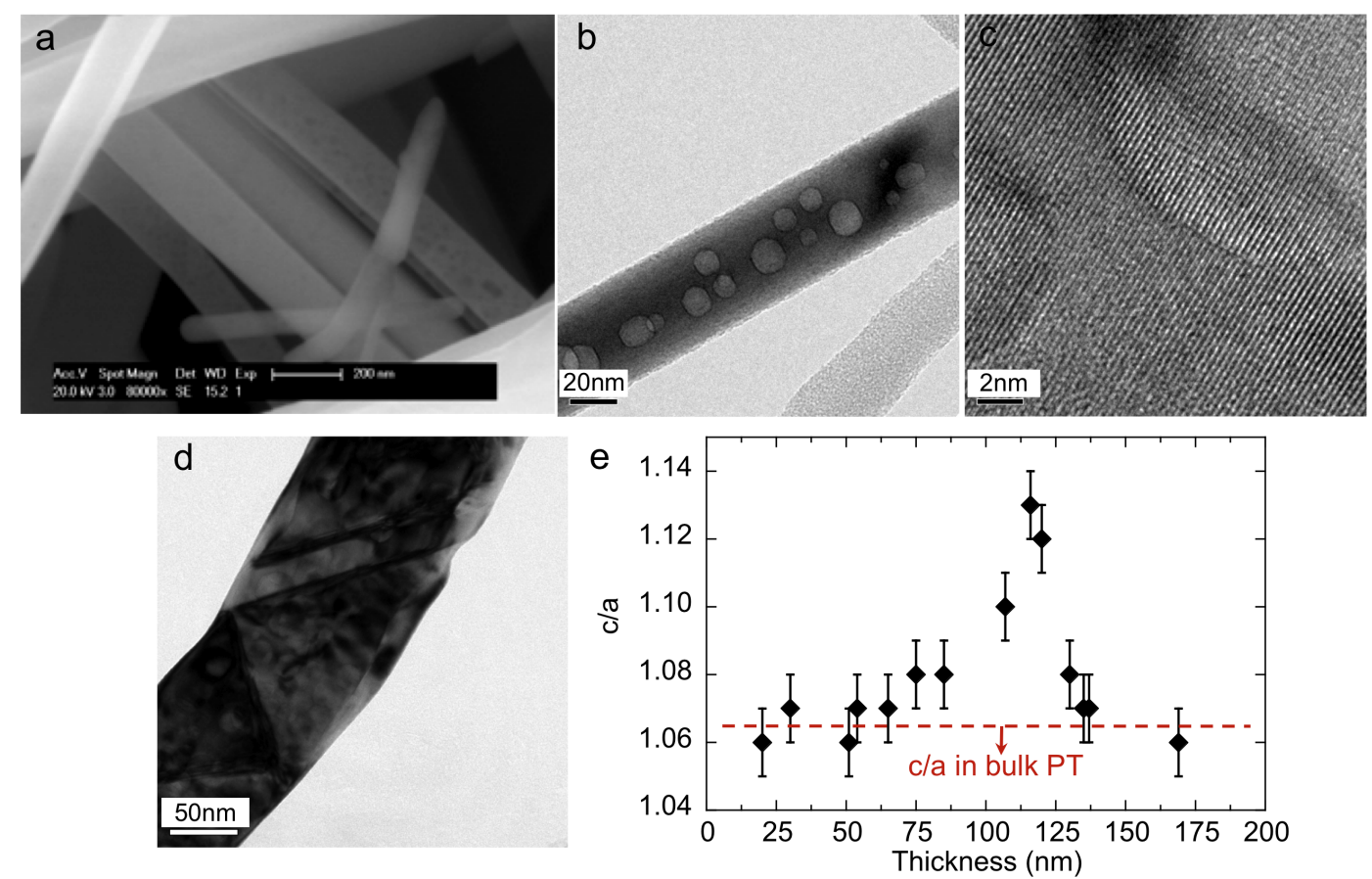

Figure A.1: SEM image of the perovskite PT nanowires. b, Low-magnification TEM image showing the closed nanosized pores in the nanowires. c, High resolution image revealing that the monocrystallinity is not affected by the pores. d, TEM image of the area with $90^{\circ}$ domains. e, $c / a$ versus the thickness of the nanowires. 
a

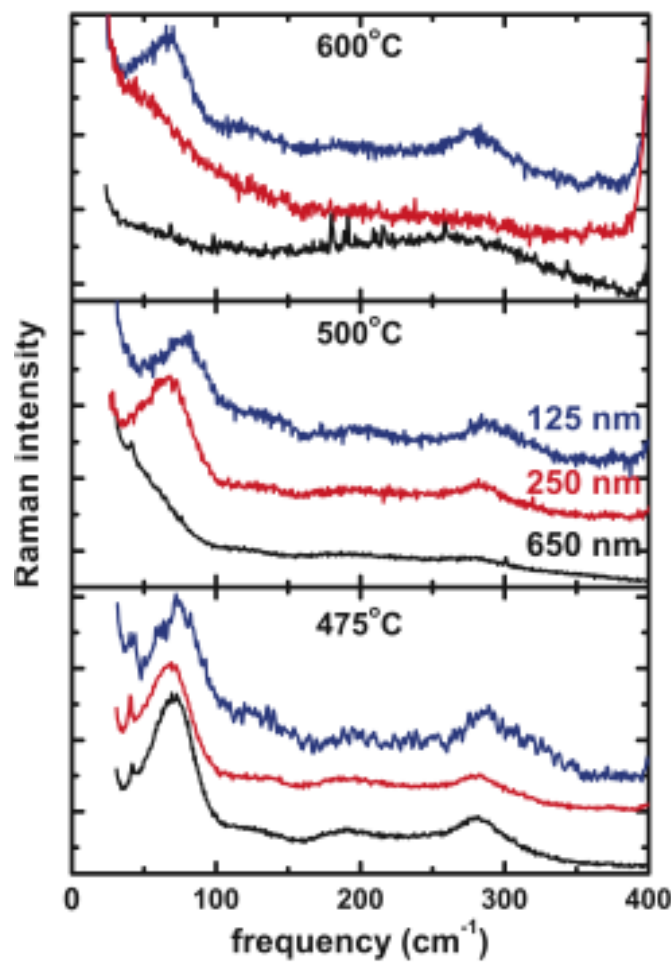

b

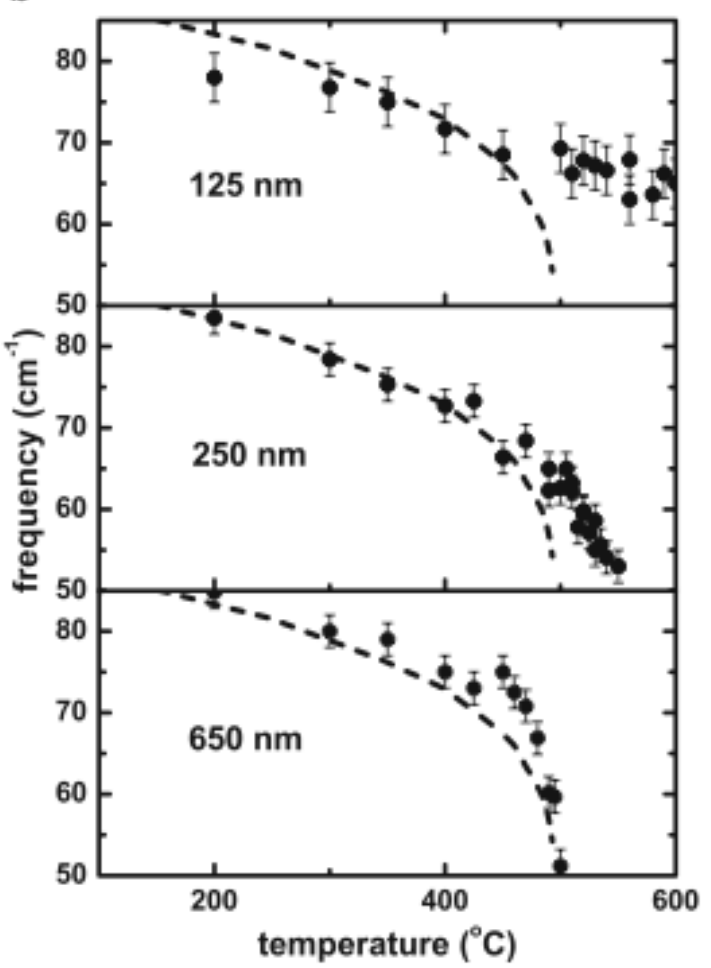

Figure A.2: Spectra on single wires of diameters 125, 250 and $650 \mathrm{~nm}$, at temperatures spanning the Curie temperature. The lowest frequency E(1TO) feature was followed to investigate the diameter-dependent Curie temperature. The occasional peak around $40-50 \mathrm{~cm}^{-1}$ is spurious, possibly from the substrate. $\mathrm{b}$, The frequency of the $\mathrm{E}(1 \mathrm{TO})$ line plotted vs temperature for the three wires, showing the line falling to $55 \mathrm{~cm}^{-1}$ before disappearing at $\mathrm{T}_{C}$. The thinner wires have a clear enhancement of the ferroelectric temperature range.

paraelectric phase transition in individual nanowires by temperature-dependent Raman spectroscopy. Figure 2a shows the Raman spectra in the low-frequency $\left(\leq 400 \mathrm{~cm}^{-1}\right)$ region for three wires with thicknesses of 650,250 and $125 \mathrm{~nm}$, at temperatures that span the bulk Curie temperature of about $490{ }^{\circ} \mathrm{C}^{x i v, x v}$. There are no Raman active modes in the paraelectric cubic phase, and as expected the thickest wire loses all Raman bands above the bulk Curie temperature. However, the signal from the ferroelectric tetragonal phase persists to higher temperatures in thinner wires, to over $600{ }^{\circ} \mathrm{C}$ in the $125 \mathrm{~nm}$ nanowire.

The E(1TO) mode below $100 \mathrm{~cm}^{-1}$ provides a convenient signature of the impending ferroelectric-paraelectric transition. In bulk PT it is a narrow line near $90 \mathrm{~cm}^{-1}$ at ambient temperature, and it softens and broadens as $\mathrm{T}_{C}$ is approached, 
finally disappering after reaching $\sim 55 \mathrm{~cm}^{-1}$ at $\mathrm{T}_{C}^{x i v}$. It is important that this is a pure mode with a frequency that is independent of the orientation of the polar axis (c axis) relative to the photon propagation direction ${ }^{x v i}$, which is necessarily uncontrolled in this experiment. Figure $2 \mathrm{~b}$ shows its frequency varying with the temperature, compared with single crystal bulk $\mathrm{PT}^{x i v}$ for comparision. The $650 \mathrm{~nm}$ nanowire shows results in a good agreement with bulk PT while the $250 \mathrm{~nm}$ nanowire exhibits the $\mathrm{E}(\mathrm{TO})$ mode frequency shifted up by $30 \mathrm{~K}$ compared to bulk $\mathrm{PT}$, implying $\mathrm{T}_{C} \approx 520^{\circ} \mathrm{C}$. The $125 \mathrm{~nm}$ nanowire has an enormously enhanced $\mathrm{T}_{C}$, with the presence of the $\mathrm{E}(1 \mathrm{TO})$ mode still seen in spectra at the $600{ }^{\circ} \mathrm{C}$ limit of our variable-temperature stage. The $125 \mathrm{~nm}$ wire is expected to have $c / a \approx 1.08$ (Fig. 1e), a $23 \%$ tetragonality enhancement. In parallel $\mathrm{T}_{C}$ is enhanced by more than $100 \mathrm{~K}$, i.e. more than $15 \%$. We have found no previous report of enhanced of ferroelectricity in freestanding nanostructures.

Figure 3 shows the lattice constants determined in a temperature-dependent XRD investigation of a multiple-wire sample, together with the standard bulk data for comparison ${ }^{x v i i, x v i i i}$. It is clearly seen that the lattice parameter $c$ and $c / a$ are increased compared to the standard bulk over the whole measured temperature range, and the Curie temperature is enhanced by about $40 \mathrm{~K}$. As shown in the supplementary material, the volume-weighted average diameter of the wires is 220-270 nm, bringing the ambient-temperature $c / a$ and the Curie temperature enhancement into agreement with the data on single wires.

It is untenable that the enhancements are related to a purely size effect, which can appear only on a much shorter length scale ${ }^{x i x, x x, x x i}$. Furthermore, the depolarising field often identified as responsible for changed ferroelectric behaviour in nanostructured ceramics leads to a reduced, rather than enhanced, order parameter and $\mathrm{T}_{C}{ }^{x x i i}$. In any case, the variable polarization direction relative to the wire axis makes these sources unlikely to give a clear trend depending merely on the thickness of the nanowires.

A clue regarding the origin of the enhanced order parameter is illustrated in Figure 4, which shows the distribution of approximately spherical voids in three typical nanowires with diameters near the most enhanced $c / a$. Combined with Fig. 1e, these data clearly show the trend that the most enhanced order parameters are closely correlated with a high void density. In particular the $115 \mathrm{~nm}$ nanowire, 

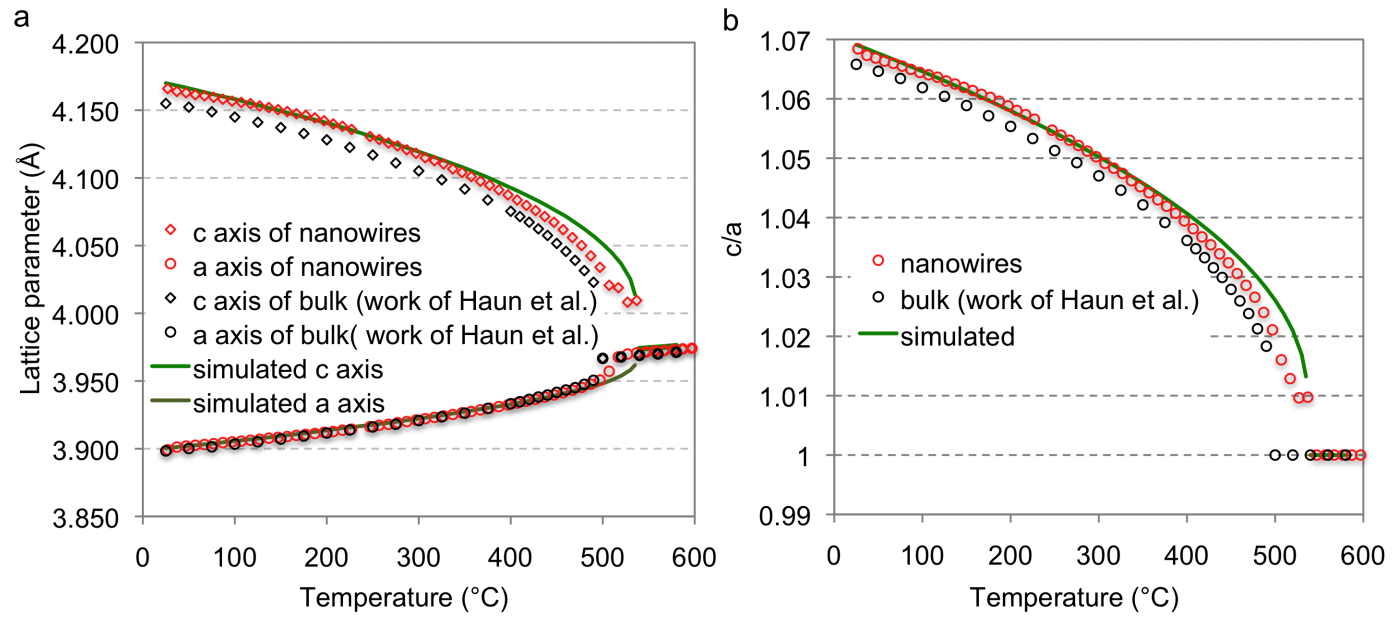

Figure A.3: Temperature dependence of lattice parameters (a) and c/a (b) of the multiple-wire sample compared with the standard bulk data (ref. 17). The simulated lattice parameters and $c / a$ of PT under $0.4 \mathrm{Gpa}$ hydrostatic tensile stress are also included (see the text for further discussions).

which shows a $c / a$ about 1.13 , shows a remarkable density of approximately $10 \mathrm{~nm}$ voids separated by about three times the diameter. As such closed pores shrink to reduce the surface energy, they impose a tensile stress on their surrounding solid media; in a solid with dense and uniformly distributed closed pores the total stress effect from all the pores is similar to that of hydrostatic tensile stress spread over the bulk part of the solid ${ }^{x x i i i}$. A continuum treatment gives void-surface stresses of $0.4-4 \mathrm{GPa}$ for a void diameter of $10 \mathrm{~nm}$ and surface tensions of 1 to $10 \mathrm{~N} / \mathrm{m}$ reported for $\mathrm{PT}$ The 0.15

It is well established that hydrostatic compressive strain reduces the Curie temperature of $\mathrm{PT}^{\text {vii,viii }}$, implying an enhanced order parameter under hydrostatic tensile strain. $\mathrm{Ab}$ initio simulations of $\mathrm{PbTiO}_{3}$ under negative pressure predict a strongly nonlinear enhancement of the tetragonality and spontaneous polarization at pressures to $-4.5 \mathrm{GPa}$ (red curve with square markers in Fig. $4 \mathrm{~d}$ ) ${ }^{i x}$. The maximal measured tetragonality, occurring in nanowires of $\approx 110 \mathrm{~nm}$ diameter is twice that of the bulk value (1.12 vs. 1.06 respectively), which corresponds to $\approx 4 \mathrm{GPa}$ tensile stress in the calculated curve of Figure 5a. The strain-stress relationship is only weakly superlinear up to $4 \mathrm{GPa}$, so that the stress leading to $c / a$ of 1.08 in a 125 $\mathrm{nm}$ diameter wire is near $2 \mathrm{GPa}$, within the 0.4-4 GPa expected from the observed void distribution. 

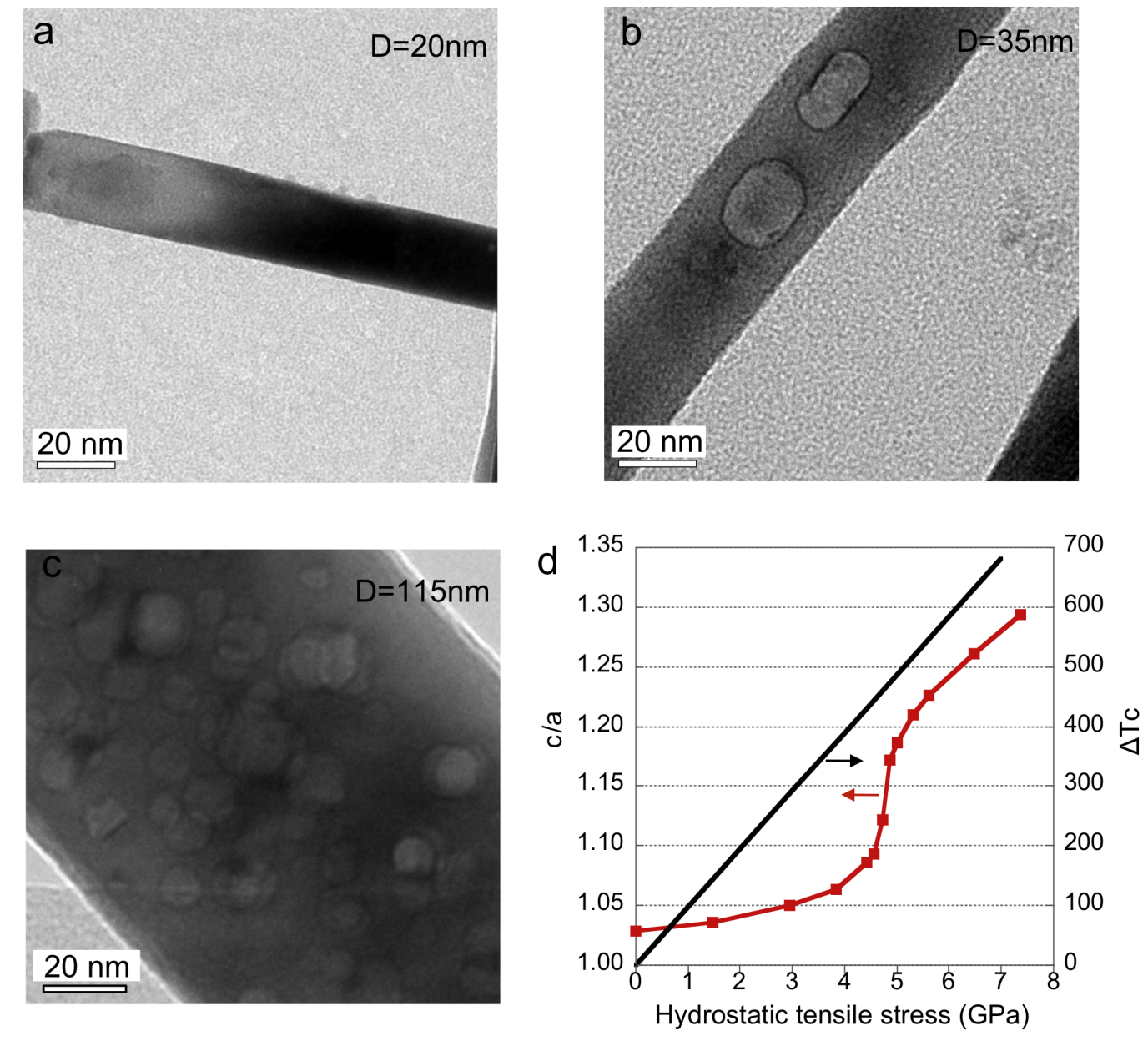

Figure A.4: a-c, TEM images showing the pore distribution in the nanowires with different thicknesses. d, Enhanced $c / a$ (red curve with square markers) of PT under hydrostatic tensile stress simulated by the first-principles calculations (the curve is reconstructed according to the data in ref. 9) and upshift of $\mathrm{T}_{C}$ (solid black curve) of PT under hydrostatic tensile stress predicted by the LGD theory. 
Within the Landau-Ginzburg-Devonshire (LGD) free energy expansion, the effect of the hydrostatic stress on the shift of Curie temperature $\Delta T_{C}$ is

$$
\Delta T_{C}=2\left(Q_{11}+2 Q_{12}\right) \sigma \epsilon_{0} C
$$

where $Q_{11}$ and $Q_{12}$ are the longitudinal and transverse electrostriction coefficients, $\sigma$ the hydrostatic stress, $\epsilon_{0}$ vacuum permittivity, and $C$ the Curie-Weiss constant. The predicted dependence of $\Delta T_{C}$ is plotted in Figure $4 \mathrm{~d}$ (solid black curve), showing a $200^{\circ} \mathrm{C}$ enhancement under the hydrostatic tensile stress of $2 \mathrm{GPa}$ implied by the $c / a$ enhancement in $125 \mathrm{~nm}$ nanowire; the data of Fig. 3d are in excellent agreement. For the $250 \mathrm{~nm}$ nanowire, the measured $\Delta T_{C}$ of $30^{\circ} \mathrm{C}$ corresponds to a hydrostatic tensile stress $\sim 0.3 \mathrm{GPa}$.

The unit cell dimensions in the presence of hydrostatic tensile stress are also described within LGD theory. A simulation of the temperature dependent lattice constants of the multiple-wire sample has been performed according to Eq. 1 and plotted as the dashed curve in Figure $3 \mathrm{~b}^{x x i v}$. The only input parameter $\sigma$ is determined to be about $0.4 \mathrm{GPa}$ from the measured shift of $\mathrm{T}_{C}$ according to Eq. 1. It is seen that the simulation has qualitatively reproduced the features of the variations of the temperature dependent lattice constants observed in multiplewire sample with respect of the stress-free bulk sample, and is almost perfect for the temperature range below $350^{\circ} \mathrm{C}$. The calculated temperature dependent $c / a$ has also been plotted (dashed curve in Fig. 3b), showing again a good match with the experimental data. The small deviation at high temperature can be attributed to the variable stress magnitude among wires of differing diameter.

It is clear that the voids are a central element in the development of the tensile stress. It is important therefore to emphasize that the voids are stable with time: samples showed the same increased tetragonality after having been stored for more than 2 years. We note that the preparation route passed through the PX phase, with the same stoichiometry as PT but with a specific volume that is larger by $15 \%$. The accumulation of voids due to phase transformation into a dense structure is known indeed to occur, e.g. upon transformation from amorphous to crystalline oxides $^{x x v}$.

In summary, we have demonstrated the enhancement of the tetragonaility and 
Curie temperature by hydrostatic tensile stress, an effective negative pressure, in nanoporous nanowires of PT, as is predicted by both ab initio modelling and semi-empirical LGD theory. The observed tetragonality is more than doubled in the nanowires with a high density of approximately $10 \mathrm{~nm}$ voids in the structure compared to the stress-free bulk sample. The tensile stress is seen clearly only in a narrow range of nanowire diameters centred near $110 \mathrm{~nm}$, but in that range it is stable for years and against cycling to at least $600^{\circ} \mathrm{C}$. A technically important implication from the observations is that effective hydrostatic tensile stress in freestanding nanowires is created by the surface tension of dense nanosized spherical pores and can reach GPa level. Such pore-engineering route can serve as a clever way to apply a negative pressure on solids without external forces. Moreover, the enhancement by negative pressure requires a positive hydrostatic electrostriction coefficient $\left(Q_{11}+2 Q_{12}\right)$ (Eq. 1) ${ }^{x x v i}$, which is satisfied in most of the perovskites. Therefore the pore-engineering route holds a promise of enhanced ferro- and piezoelectric properties and extended working temperature range, especially appealing for lead-free perovskite ferroelectrics, whose bulk properties are inferior so far in comparison with classical lead-containing ferroelectrics.

\section{A.4 References of this appendix}

${ }^{i}$ Haertling, G. H., J. Am. Ceram. Soc. 82, 797 (1999).

${ }^{i i}$ Garcia V et al., Nature 460, 81 (2009).

${ }^{i i i}$ Baek S. et al., Science 334, 958 (2011).

${ }^{i v}$ Pertsev N. A., Zembilgotov A. G., Tagantsev A. K., Phys. Rev. Lett. 84, 1988 (1998).

${ }^{v}$ Streiffer S. K., et al., Phys. Rev. Lett. 89, 067601 (2002).

${ }^{v i}$ Choi, K. J. et al., Science 306, 1005 (2004).

${ }^{v i i}$ Nelmes R. J., Katrusiak A., J. Phys. C: Solid State Phys. 19, L725 (1986).

${ }^{v i i i}$ Ramrez R., Lapena M. F., Gonzalo J. A., Phys. Rev. B. 42, 2604 (1990).

${ }^{i x}$ Tinte S., Rabe K. M., Vanderbilt D., Phys. Rev. B. 68, 144105 (2003).

${ }^{x}$ Cheng H. M., et al., J. Am. Ceram. Soc. 75, 1123 (1992).

${ }^{x i}$ Wang J., et al., Chem. Mater. 23, 2529 (2011).

${ }^{x i i}$ Mabud S. A., Glazer A. M., J. Appl. Phys. 62, 49 (1979). 
${ }^{x i i i}$ Abrahams S. C., Kurtz S. K., Jamieson P. B., Phys. Rev. 172, 551 (1968).

${ }^{x i v}$ Burns G., Scott B. A., Phys. Rev. B 7, 3088 (1973).

${ }^{x v}$ Fontana M. D., Idrissi H., Kugel G. E., Wojcik K., J. Phys.: Condens. Matter 3, 8695 (1991).

${ }^{x v i}$ Foster C. M., Li Z., Grimsditch M., Chan S.-K., Lam D. J., Phys. Rev. B 48, 10160 (1993).

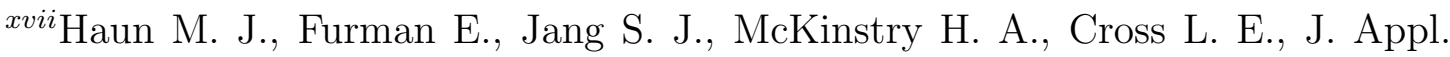
Phys. 62, 3331 (1987).

${ }^{\text {xviii }}$ The temperature dependence of lattice parameters measured on $\mathrm{PbTiO}_{3}$ single crystal (ref. 12) is in good agreement with that the data measured on $\mathrm{PbTiO}_{3}$ bulk powders (ref. 17), thus we only show the data from ref. 17 as the reference for bulk $\mathrm{PbTiO}_{3}$.

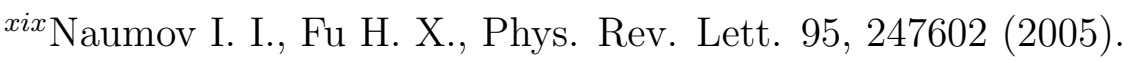

${ }^{x x}$ Geneste G., Bousquet E., Junquera J., Ghosez P., Appl. Phys. Lett. 88, 112906 (2006).

${ }^{x x i}$ Spanier J. E., et al., Nano Lett. 6, 735 (2006).

xxii Junquera J., Ghosez P., Nature 422, 506 (2003).

${ }^{x x i i i}$ Mackenzie J. K. Proc. Phys. Soc. B 63, 2 (1950).

${ }^{x x i v}$ See the supporting materials for the details of the simulation. The coefficients of the free energy expansion of $\mathrm{PbTiO}_{3}$ are taken from ref. 17.

${ }^{x x v}$ R. Nakamura, et al. Scripta Materialia 66, 182 (2012).

${ }^{x x v i}$ This is valid for other symmetries of the ferroelectric phase (e.g. rhombohedral) to which the cubic paraelectric phase transforms into, as both the quadratic terms and the electrostriction terms in the free energy expansion are isotropic with respect to the polarization orientation. 
A.4. References of this appendix 


\section{Appendix B}

\section{Semi-classical equation describing resonant Raman scattering}

The Raman cross section is directly proportional to the Raman polarisability. The equation below comes from quantum perturbation theory, and is the expression for an element of the Raman polarisability tensor between quantum states $|i\rangle$ and $|f\rangle$ - i.e., the Raman polarisability of a single vibrational mode at a single orientation. Of course, spectra that we measure are sums over all activated modes, and over whatever orientations are collected geometrically by the experimental apparatus. The subscripts $k$ and $l$ indicate spatial axes i.e. one of $x, y, z$. The subscripts $i$ and $f$ refer to the quantum state corresponding with the initial and final states that describe the Raman scattering event, and the subscript $r$ refers to some other complete quantum state. The sum is performed over every complete quantum state which is not the final or initial one.

$$
\alpha_{k l}=\frac{1}{\hbar} \sum_{r \neq i, f}\left\{\frac{\left\langle f\left|p_{k}\right| r\right\rangle\left\langle r\left|p_{l}\right| i\right\rangle}{\left(\omega_{r}-\omega_{i}\right)-\omega_{L}-i \Gamma_{r}}+\frac{\left\langle f\left|p_{l}\right| r\right\rangle\left\langle r\left|p_{k}\right| i\right\rangle}{\left(\omega_{r}-\omega_{f}\right)-\omega_{L}-i \Gamma_{r}}\right\}
$$

where $\alpha$ is the Raman polarisability tensor, $\omega$ are frequencies of the various complete quantum states, $\omega_{L}$ is the frequency of the excitation laser, $p$ are quantum mechanical dipole moment operators and $\Gamma$ is a damping term that arises from 
finite state lifetimes.

For the purpose of understanding resonant Raman scattering, we need consider only the denominators inside the sum. When the frequency gap between two given states, $\left(\omega_{r}-\omega_{i}\right)$ and/or $\left(\omega_{r}-\omega_{f}\right)$ is very similar to the frequency of the laser, $\omega_{L}$, then the denominator term vanishes towards zero. This causes the element in the summation, and therefore the polarisability for this mode excited at this frequency to become very large. In simple terms, this occurs when the energy of the wavelength is very close to the energy of allowed transitions in the material - most commonly, these are electronic transitions between the valence and conduction bands. So this is the source of the resonant Raman enhancement[34]. 


\section{Bibliography}

[1] J. H. Haeni, P. Irvin, W. Chang, R. Uecker, P. Reiche, Y. L. Li, S. Choudhury, W. Tian, M. E. Hawley, B. Craigo, A. K. Tagantsev, X. Q. Pan, S. K. Streiffer, L. Q. Chen, S. W. Kirchoefer, J. Levy, and D. G. Schlom, "Room-temperature ferroelectricity in strained $\mathrm{SrTiO}_{3}$," Letters to Nature, vol. 430, p. 758, 2004.

[2] J. Toulouse, P. DiAntonio, B. E. Vugmeister, X. M. Wang, and L. A. Knauss, "Precursor Effects and Ferroelectric Macroregions in $\mathrm{KTa}_{1-\mathrm{x}} \mathrm{Nb}_{\mathrm{x}} \mathrm{O}_{3}$ and $\mathrm{K}_{1-\mathrm{y}} \mathrm{Li}_{\mathrm{y}} \mathrm{TaO}_{3}$," Physical Review Letters, vol. 68, p. 232, 1992.

[3] J. Wang, K. Schenk, A. Carvalho, B. Wylie-van Eerd, J. Trodahl, C. S. Sandu, M. Bonin, I. Gregora, Z. He, T. Yamada, H. Funakubo, P. R. Briddon, and N. Setter, "Structure Determination and Compositional Modification of Body-Centered Tetragonal PX-Phase Lead Titanate," Chemistry of Materials, vol. 23, p. 2529, 2011.

[4] B. W. van Eerd, D. Damjanovic, N. Klein, N. Setter, and J. Trodahl, "Structural complexity of $\left(\mathrm{Na}_{0.5} \mathrm{Bi}_{0.5}\right) \mathrm{TiO}_{3}-\mathrm{BaTiO}_{3}$ as revealed by Raman spectroscopy," Physical Review B, vol. 82, p. 104112, 2010.

[5] L.-Y. Li and X.-G. Tang, "Effect of electric field on the dielectric properties and ferroelectric phase transition of sol-gel derived $\left(\mathrm{Ba}_{0.90} \mathrm{Ca}_{0.10}\right) \mathrm{TiO}_{3}$ ceramics," Materials Chemistry and Physics, vol. 115, p. 507, 2009.

[6] B. Noheda, J. A. Gonzalo, L. E. Cross, R. Guo, S.-E. Park, D. E. Cox, and G. Shirane, "Tetragonal-to-monoclinic phase transition in a ferroelectric perovskite: The structure of $\mathrm{PbZr}_{0.52} \mathrm{Ti}_{0.48} \mathrm{O}_{3}$," Physical Review B, vol. 61, p. 8687, 2003. 
[7] T. R. Shrout and S. J. Zhang, "Lead-free piezoelectirc ceramics: Alternatives for PZT?," Journal of Electroceramics, vol. 19, p. 111, 2007.

[8] J. Ricote, R. W. Whatmore, and D. J. Barber, "Studies of the ferroelectric domain configuration and polarization of rhombohedral PZT ceramics," Journal of Physics: Condensed Matter, vol. 12, p. 323, 2000.

[9] E. Cross, "Lead-free at last," Nature, vol. 432, p. 24, 2004.

[10] A. M. Intelligence, World Piezoelectric Device Market. Acmite Market Intelligence, 2011.

[11] B. A. Fowler, G. F. Nordberg, M. Nordberg, and L. Friberg, Handbook on the Toxicology of Metals, third edition. Academic Press, 2011.

[12] S. Ganesan and M. G. Pecht, Lead-free electronics. WILEY-interscience, 2006.

[13] Y. Kuroiwa, S. Aoyagi, and A. Sawada, "Evidence for Pb-O Covalency in Tetragonal $\mathrm{PbTiO}_{3}, "$ Physical Review Letters, vol. 87, p. 217601, 2001.

[14] J. F. Scott, "Soft-mode spectroscopy: Experimental studies of structural phase transitions," Reviews of Modern Physics, vol. 46, p. 83, 1974.

[15] Fleury, Paul A, "The effects of soft modes on the structure and properties of materials," Annual Review of Materials Science, vol. 6, no. 1, pp. 157-180, 1976.

[16] T. Takenaka, K. ichi Maruyama, and K. Sakata, " $\left(\mathrm{Bi}_{1 / 2} \mathrm{Na}_{1 / 2}\right) \mathrm{TiO}_{3}-\mathrm{BaTiO}_{3}$ System for Lead-Free Piezoelectric Ceramics," Japanese Journal of Applied Physics, vol. 30, p. 2236, 1991.

[17] K. Kato, S. Tsukada, J. Kano, T. Yamamoto, and S. Kojima, "Raman Scattering Study of Ca-Modified Lead Titanate," IEEE Transactions on Ultrasonics, Ferroelectrics, and Frequency Control, vol. 54, p. 2599, 2007.

[18] A. Yamanaka, M. Kataoka, Y. Inaba, K. Inoue, B. Hehlen, and E. Courtens, "Evidence for competing orderings in strontium titanate from hyper-Raman scattering spectroscopy," Europhysics letters, vol. 50, p. 688, 2000. 
[19] R. Ouillon, J.-P. Pinan-Lucarre, P. Ranson, P. Pruzan, S. J. Mishra, R. Ranjan, and D. Pandey, "A Raman scattering study of the phase transitions in $\mathrm{SrTiO}_{3}$ and in the mixed system $\left(\mathrm{Sr}_{1-\mathrm{x}} \mathrm{Ca}_{\mathrm{x}}\right) \mathrm{TiO}_{3}$ at ambient pressure from $\mathrm{T}=300 \mathrm{~K}$ down to $8 \mathrm{~K}$," Journal of Physics: Condensed Matter, vol. 14, p. 2079, 2002.

[20] A. Vasudevarao, A. Kumar, L. Tian, J. H. Haeni, Y. L. Li, C. Eklund, Q. X. Jia, R. Uecker, P. Reiche, K. M. Rabe, L. Q. Chen, D. G. Schlom, and V. Gopalan, "Multiferroic Domain Dynamics in Strained Strontium Titanate," Physical Review Letters, vol. 97, p. 257602, 2006.

[21] M. Itoh, R. Wang, Y. Inaguma, T. Yamaguchi, Y.-J. Shan, and T. Nakamura, "Ferroelectricity Induced by Oxygen Isotope Exchange in Strontium Titanate Perovskite," Physical Review Letters, vol. 82, p. 3540, 1999.

[22] J. Petzelt, T. Ostapchuk, I. Gregora, P. Kuzel, J. Liu, and Z. Shen, "Infrared and Raman studies of the dead grain-boundary layers in $\mathrm{SrTiO}_{3}$ fine-grain ceramics," Journal of Physics: Condensed Matter, vol. 19, p. 196222, 2007.

[23] W. Zhong and D. Vanderbilt, "Effect of quantum fluctuations on structural phase transitions in $\mathrm{SrTiO}_{3}$ and $\mathrm{BaTiO}_{3}$," Physical Review B, vol. 53, p. 5047, 1996.

[24] N. A. Pertsev, A. K. Tagantsev, and N. Setter, "Phase transitions and straininduced ferroelectricity in $\mathrm{SrTiO}_{3}$ epitaxial thin films," Physical Review B, vol. 61, p. $825,2000$.

[25] D. A. Tenne, P. Turner, J. D. Schmidt, M. Biegalski, Y. L. Li, L. Q. Chen, A. Soukiassian, S. Trolier-McKinstry, D. G. Schlom, X. X. Xi, D. D. Fong, P. H. Fuoss, J. A. Eastman, G. B. Stephenson, C. Thompson, and S. K. Streiffer, "Ferroelectricity in Ultrathin $\mathrm{BaTiO}_{3}$ Films: Probing the Size Effect by Ultraviolet Raman Spectroscopy," Physical Review Letters, vol. 103, no. 17, p. 177601, 2009.

[26] H. Uwe and T. Sakudo, "Stress-induced ferroelectricity and soft phonon modes in $\mathrm{SrTiO}_{3}$," Physical Review B, vol. 13, p. 271, 1976.

[27] W. G. Nilsen and J. G. Skinner, "Raman Spectrum of Strontium Titanate," Journal of Chemical Physics, vol. 48, p. 2240, 1968. 
[28] F. W. Lytle, "X-Ray Diffractometry of Low-Temperature Phase Transformations in Strontium Titanate," Journal of Applied Physics, vol. 35, p. 2212, 1964 .

[29] C. Ang, L. E. Cross, Z. Yu, R. Guo, A. S. Bhalla, and J. H. Hao, "Dielectric loss and defect mode of $\mathrm{STiO}_{3}$ thin films under direct-current bias," Applied Physics Letters, vol. 78, p. 2754, 2001.

[30] A. A. Sirenko, C. Bernhard, A. Golnik, A. M. Clark, J. Hao, W. Si, and X. X. $\mathrm{Xi}$, "Soft-mode hardening in $\mathrm{SrTiO}_{3}$ thin films," Nature, vol. 404, p. 373, 2000 .

[31] D. Fuchs, C. W. Schneider, R. Schneider, and H. Rietschel, "High dielectric constant and tunability of epitacial $\mathrm{SrTiO}_{3}$ thin film capacitors," Journal of Applied Physics, vol. 85, p. 7362, 1999.

[32] M. D. Biegalski, Y. Jia, D. G. Schlom, S. Trolier-McKinstry, S. K. Streiffer, V. Sherman, R. Uecker, and P. Reiche, "Relaxor ferroelectricity in strained epitaxial $\mathrm{SrTiO}_{3}$ thin films on $\mathrm{DyScO}_{3}$ substrates," Applied Physics Letters, vol. 88, p. 192907, 2006.

[33] D. A. Tenne, A. Bruchhausen, N. D. Lanzillotti-Kimura, A. Fainstein, R. S. Katiyar, A. Cantarero, A. Soukiassian, V. Vaithyanathan, J. H. Haeni, W. Tian, D. G. Schlom, K. J. Choi, D. M. Kim, C. B. Eom, H. P. Sun, X. Q. Pan, Y. L. Li, L. Q. Chen, Q. X. Jia, S. M. Nakhmanson, K. M. Rabe, and X. X. Xi, "Probing Nanoscale Ferroelectricity by Ultraviolet Raman Spectroscopy," Science, vol. 313, p. 1614, 2006.

[34] E. C. Le Ru and P. G. Etchegoin, Principles of Surface-Enhanced Raman Spectroscopy and related plasmonic effects. Elsevier Science Limited, 2008.

[35] W. Taylor and A. F. Murray, "Tetragonal $\mathrm{SrTiO}_{3}$ revisited: The effect of impurities on the Raman spectrum," Solid State Communications, vol. 31, p. 937, 1979 .

[36] J. Petzelt, I. Gregora, I. Rychetskỳ, T. Ostapchuk, S. Kamba, P. Vaněk, Y. Yuzyuk, A. Almeida, M. Chavez, B. Gorshunov, M. Dressel, S. HoffmainEifert, and R. Waser, "Polar grain boundaries in undoped $\mathrm{SrTiO}_{3}$ ceramics," Journal of the European Ceramic Society, vol. 21, p. 2681, 2001. 
[37] P. A. Fleury, J. F. Scott, and J. M. Worlock, "Soft phonon modes and the $110^{\circ} \mathrm{K}$ phase transition in $\mathrm{SrTiO}_{3}, "$ Physical Review Letters, vol. 21, p. 16, 1968.

[38] H. Taniguchi, T. Yagi, M. Takesada, and M. Itoh, "Isotope effect on the softmode dynamics of $\mathrm{SrTiO}_{3}$ studies by Raman scattering," Physical Review B, vol. 72 , p. 064111, 2005.

[39] H. Uwe, H. Yamaguchi, and T. Sakudo, "Ferroelectric microregion in $\mathrm{KTa}_{1-\mathrm{x}} \mathrm{Nb}_{\mathrm{x}} \mathrm{O}_{3}$ and $\mathrm{SrTiO}_{3}, "$ Ferroelectrics, vol. 96, p. 123, 1989.

[40] Y. L. Du, G. Chan, and M. S. Zhang, "Investigation of structural phase transition in polycrystalline $\mathrm{SrTiO}_{3}$ thin films by Raman spectroscopy," Solid State Communications, vol. 130, p. 577, 2004.

[41] U. Fano, "Effects of configuration interaction on intensities and phase shifts," Physical Review, vol. 124, p. 1866, Dec 1961.

[42] H. W. Jang, A. Kumar, S. Denev, M. D. Biegalski, P. Maksymovych, C. W. Bark, C. T. Nelson, C. M. Folkman, C. M. Brooks, D. A. Tenne, D. G. Schlom, L. Q. Chen, X. Q. Pan, S. V. Kalinin, V. Gopalan, and C. B. Eom, "Ferroelectricity in Strain-Free $\mathrm{SrTiO}_{3}$ Thin Films," Physical Review Letters, vol. 104, p. 197601, 2010.

[43] U. Bianchi, W. Kleemann, and J. G. Bednorz, "Raman scattering of ferroelectric $\mathrm{Sr}_{1-\mathrm{x}} \mathrm{Ca}_{\mathrm{x}} \mathrm{TiO}_{3}, \mathrm{x}=0.007$," Journal of Physics, Condensed Matter, vol. 6, p. 1229, 1994.

[44] T. Ostapchuk, J. Petzelt, V. Železnỳ, A. Pashkin, J. Pokornỳ, I. Drbohlav, R. Kužel, D. Rafaja, B. Gorshunov, M. Dressel, C. Ohly, S. Hoffman-Eifert, and R. Waser, "Origin of soft-mode stiffening and reduced dielectric response in $\mathrm{SrTiO}_{3}$ thin films," Physical Review B, vol. 66, p. 235406, 2002.

[45] D. A. Crandles, B. Nicholas, C. Dreher, C. C. Homes, A. W. McConnell, B. P. Clayman, W. H. Gong, and J. E. Greedan, "Optical properties of highly reduced $\mathrm{SrTiO}_{3-\mathrm{x}}$, " Physical Review B, vol. 59, p. 12842, 1999.

[46] H. Uwe, K. B. Lyons, H. L. Carter, and P. A. Fleury, "Ferroelectric microregions and Raman scattering in $\mathrm{KTaO}_{3}$," Physical Review B, vol. 33, p. 6436, 1986. 
[47] J. G. Bednorz and K. A. Müller, " $\mathrm{Sr}_{1-x} \mathrm{Ca}_{\mathrm{x}} \mathrm{TiO}_{3}$ : An XY Quantum Ferroelectric with Transition to Randomness," Physical Review Letters, vol. 52, p. 2289, 1984.

[48] W. Kleeman, A. Albertini, M. Kuss, and R. Lindner, "Optical detection of symmetry breaking on a nanoscale in $\mathrm{SrTiO}_{3}$ :Ca," Ferroelectrics, vol. 203, p. $57,1997$.

[49] M. Itoh, R. Wang, M. Naraharo, and T. Kyomen, "Dielectric Properties of $\mathrm{SrTi}^{18} \mathrm{O}_{3}, "$ Ferroelectrics, vol. 285, p. 3, 2003.

[50] D. A. Tenne, A. K. Farrar, C. M. Brooks, T. Heeg, J. Schubert, H. W. Jang, C. W. Bark, C. M. Folkman, C. B. Eom, and D. G. Schlom, "Ferroelectricity in nonstoichiometric $\mathrm{SrTiO}_{3}$ films studied by ultraviolet Raman spectroscopy," Applied Physics Letters, vol. 97, p. 142901, 2010.

[51] Y. Y. Guo, H. M. Liu, D. P. Yu, and J. Liu, "Ferroelectricity and superparamagnetism in $\mathrm{Sr} / \mathrm{Ti}$ nonstoichiometric $\mathrm{SrTiO}_{3}$," Physical Review B, vol. 85, p. 104108, 2012.

[52] A. A. Sirenko, I. A. Akimov, J. R. Fox, A. M. Clark, H.-C. Li, W. Si, and X. X. Xi, "Observation of the First-Order Raman Scattering in $\mathrm{SrTiO}_{3}$ Thin Films," Physical Review Letters, vol. 82, p. 4500, 1999.

[53] D. A. Tenne, I. E. Gonenli, A. Soukiassian, D. G. Schlom, S. M. Nakhmanson, K. M. Rabe, and X. X. Xi, "Raman study of oxygen reduced and re-oxidized strontium titanate," Physical Review B, vol. 76, p. 024303, 2007.

[54] Y. S. Kim, D. J. Kim, T. H. Kim, T. W. Noh, J. S. Choi, B. H. Park, and J. Yoon, "Observation of room-temperature ferroelectricity in tetragonal strontium titanate thin films on $\mathrm{SrTiO}_{3}$ (001) substrates," Applied Physics Letters, vol. 91, p. 042908, 2007.

[55] Y. S. Kim, J. Kim, S. J. Moon, W. S. Choi, Y. W. J. Chang, J. Yu, J. Chung, and T. W. Noh, "Localized electronic states induced by defects and possible origin of ferroelectricity in strontium titanate thin films," Applied Physics Letters, vol. 94, p. 202906, 2009.

[56] P. A. Fleury and J. M. Worlock, "Electric-Field-Induced Raman Scattering in $\mathrm{SrTiO}_{3}$ and $\mathrm{KTaO}_{3}$," Physical Review, vol. 174, p. 174613, 1968. 
[57] M. Stengel and N. A. Spaldin, "Origin of the dielectric dead layer in nanoscale capacitors," Nature, vol. 443, p. 679, 2006.

[58] D. Bäuerle, D. Wagner, M. Wöhlecke, B. Dorner, and H. Kraxenberger, "Soft Modes in Semiconducting $\mathrm{SrTiO}_{3}$ : II. The Ferroelectric Mode," Zeitschrift für Physik B - Condensed Matter, vol. 38, p. 335, 1980.

[59] I. I. Akimov, A. A. Sirenko, A. M. Clark, J. Hao, and X. X. Xi, "Electric-FieldInduced Soft-Mode Hardening in $\mathrm{SrTiO}_{3}$ Films," Physical Review Letters, vol. 84 , p. $4625,2000$.

[60] T. Shinegari, K. Abe, K. Yamashita, T. Takemoto, R. Wang, and M. Itoh, "Raman Spectra of Ferroelectric Soft Mode in $\mathrm{SrTiO}_{3}$ : Effect of Electric Field," Ferroelectrics, vol. 285, p. 41, 2003.

[61] H. M. Cheng, J. M. Ma, Z. G. Zhao, D. Qiang, Y. X. Li, and X. Yao, "Hydrothermal synthesis of acicular lead titanate fine powders," Journal of the American Ceramic Society, vol. 75, p. 1123, 1992.

[62] R. Ranjan and A. Dviwedi, "Structure and dielectric properties of $\left(\mathrm{Na}_{0.50} \mathrm{Bi}_{0.501-\mathrm{x}} \mathrm{Ba}_{\mathrm{x}} \mathrm{TiO}_{3}: 0 \leq x \leq 0.10, "\right.$ Solid State Communications, vol. 135, p. 394, 2005.

[63] S.-T. Zhang, A. B. Kounga, E. Aulbach, T. Granzow, W. Ko, H.-J. Kleeme, and J. Rödel, "Lead-free piezoceramics with giant strain in the system $\mathrm{Bi}_{0.5} \mathrm{Na}_{0.5} \mathrm{TiO}_{3}-\mathrm{BaTiO}_{3}-\mathrm{K}_{0.5} \mathrm{Na}_{0.5} \mathrm{NbO}_{3}$. i. structure and room termperature properties," Journal of Applied Physics, vol. 103, p. 034107, 2008.

[64] B. Güttler, B. Mihailova, R. Stosch, U. Bismayer, and M. Gospodinov, "Local phenomena in relaxor-ferroelectric $\mathrm{PbSc}_{0.5} \mathrm{~B}^{\prime \prime}{ }_{0.5} \mathrm{O}_{3}(\mathrm{~B} "=\mathrm{Nb}, \mathrm{Ta})$ studied by Raman spectroscopy," Journal of Molecular Structure, vol. 661, p. 469, 2003.

[65] W. Liu and X. Ren, "Large Piezoelectric Effect in Pb-Free Ceramics," Physical Review Letters, vol. 103, p. 257602, 2009.

[66] N. E. Tornberg and C. H. Perry, "Temperature Dependent Optical Phonons in Lead Titanate," Journal of Chemical Physics, vol. 53, p. 2946, 1970. 